PNL-7185

UC-402

.$+\frac{1}{1}$

\title{
MESORAD Dose Assessment of the Chernobyl Reactor Accident
}

J. V. Ramsdell

J. M. Hubbe

G. F. Athey

W. E. Davis

December 1989

Prepared for the U.S. Department of Energy under Contract DE-AC06-76RLO 1830

Pacific Northwest Laboratory

Operated for the U.S. Department of Energy

by Battelle Memorial Institute 


\title{
DISCLAIMER
}

This program was prepared as an account of work sponsored by an agency of the United States Government. Neither the United States Covernment nor any agency thereof, nor Battelle Memorial Institute, nor any of their employees, makes any warranty, express or implied, or assumes any legal liability or responshility for the accuracy, completeness, of usefulness of any informatton, apparatus, product, or process disclosed, or represents that its wse would not infringe prtvatety owned rights. Reference herein to any specific commerical product, process, or service by trade name, trademark, manufacturer, or otherwise, does not necessarily constitute or imply its endorsement, recommendation, or favoring by the United States Government or any agency thereot, or Battelle Memorial institute. The views and opinions of authors expressed herein do not necessarily state or reflect those of the United States Government or any agency thereof.

\section{PACIFIC NORTHWEST LABORATORY operated by \\ BATTELIE MEMORIAL INSTITUTE for the \\ UNITED STATES DEPARTMENT OF ENERGY \\ under Contract DE-ACO6-76RLO 1830}

\author{
Printed in the United States of America \\ Available from \\ National Technical Information Service \\ United Sutes Deparment of Commerce \\ 5285 Port Royal Road \\ Springfield, Virginia 22161 \\ NTIS Price Codes \\ Microfiche A01 \\ Printed Copy
}

$\begin{array}{cc}\text { Pages } & \begin{array}{c}\text { Price } \\ \text { Codes }\end{array} \\ 001-025 & \text { A02 } \\ 026-050 & \text { A03 } \\ 051-075 & \text { A04 } \\ 076-100 & \text { A05 } \\ 101-125 & \text { A06 } \\ 126-150 & \text { A07 } \\ 151-175 & \text { A08 } \\ 176-200 & \text { A09 } \\ 201-225 & \text { A10 } \\ 226-250 & \text { A11 } \\ 251-275 & \text { A12 } \\ 276-300 & \text { A13 }\end{array}$




\section{MESORAD DOSE ASSESSMENT OF THE CHERNOBYL REACTOR ACCIDENT}
J. V. Ramsde 11
J. M. Hubbe
G. F. Athey
W. E. Davis

December 1989

Prepared for

the U.S. Department of Energy under Contract DE-AC06-76RLO 1830

Pacific Northwest Laboratory Richland, Washington 99352 


\section{SUMMARY}

Shortly after midnight local time on April 26, 1986, an accident involving Unit 4 of the Chernobylskaya Atomic Energy 5tation resulted in the release of a large amount of radioactive material to the atmosphere, which continued through May 5, 1986. This report describes the results of an assessment of the doses near the site (within $80 \mathrm{~km}$ ) made at the Pacific Northwest Laboratory using the MESORAD Dose Assessment model. The MESORAD model was developed for emergency response applications for the U.S. Nuclear Regulatory Commission and the U.S. Department of Energy, Richland Operations Office.

Winds during the release period initially carried the radioactive effluents to the west and northwest. On the third day, the winds shifted to carry the efflicent to the northeast, and then on the fourth day of the release they shifted to carry the effluents to the south. As a result, other than narrow bands of relatively high contamination to the west and northwest of the reactor site, the contamination resulting from the accident was well distributed around the site.

The area encompassed by the MESORAD model domain is $10678 \mathrm{mi}^{2}$. By the beginning of May 7, 1986, the MESORAD assessment indicates that approximately $21.4 \%$ of the land within the domain had accumulated a whole body dose of $1 \mathrm{rem}$ or mone and about $1.5 \%$ of the land had accumulated a dose of 5 rem or more. MESOR, 10 further indicates that the residual radiation from ground contamination at this time was $1 \mathrm{mrem} / \mathrm{h}$ or more over almost $70 \%$ of the domain and was $10 \mathrm{mr}: \mathrm{m} / \mathrm{h}$ or greater over almost $6 \%$ of the domain. The maximum doses and dose rates predicted by MESORAD at $00 Z$ on May 7,1986 , are 
Receptor Location (Bearing/Distance in Miles)

\begin{tabular}{lccc}
\cline { 2 - 3 } & $\frac{315 / 4.7}{9.9}$ & $\frac{256 / 41.2}{20}$ & $\frac{207 / 7.5}{6.3}$ \\
Total Whole Body (rem) & & 5.9 & \\
$\begin{array}{l}\text { Whole Body Source (rem) } \\
\quad \text { Plume External }\end{array}$ & 0.09 & 0.14 & 0.11 \\
$\quad$ Ground Shine & 7.4 & 4.90 & 3.70 \\
$\quad$ Inhalation & 2.4 & 0.77 & 2.40 \\
$\begin{array}{l}\text { Dose Conmitment (rem) } \\
\quad \text { Adult Thyroid }\end{array}$ & & & \\
$\quad$ Lung & 53.0 & 98.0 & 35.0 \\
Res idual Dose Rate & 93.0 & 26.0 & 93.0 \\
$\quad$ (mrem/h) & 34.0 & 14.0 & 39.0
\end{tabular}

The total whole body dose is the sum of external doses from the passing plume and shine from ground contamination and a 50-year dose commitment from inhaled radionuclides. The thyroid and lung doses are also 50-year dose comnitments resulting from inhaled radionuclides.

The May 7 dose rate pattern is similar to a pattern of gamna-exposure rates observed on May 29. The general agreement between the two patterns is an indication that the assumptions made with regard to the Chernobyl source characteristics and meteorological conditions during the releases were reasonable. Where the patterns are different, the differences are entirely within the limits of the uncertainty of MESORAD. 


\section{CONTENTS}

SUMMARY ............................... . .

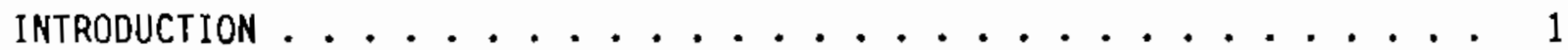

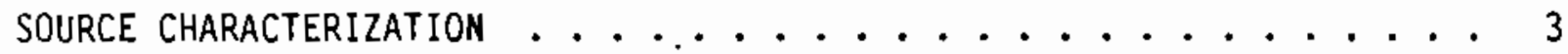

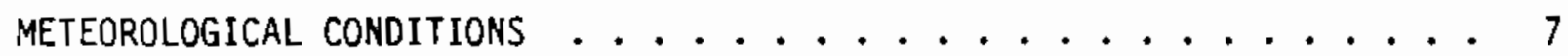

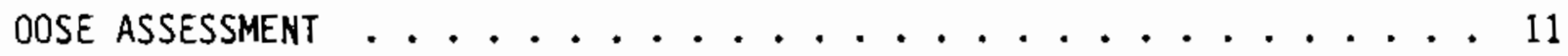

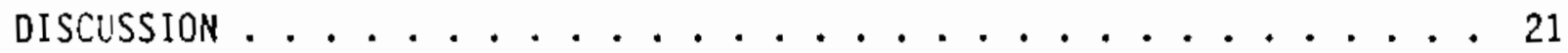

MESORAD MODEL UNCERTAINTIES . . . . . . . . . . . . . 25

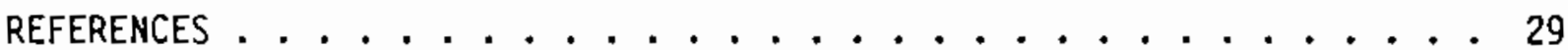

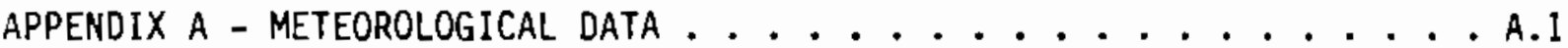

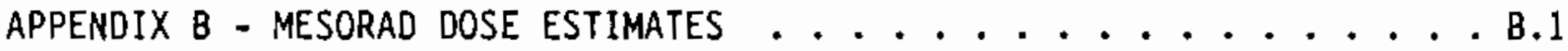




\section{FIGURES}

1 Representation of the Initial Release Configuration ....... 4

2 MESORAD Model Domain and Meteorological Observation Locations ..................... 8

3 Effect of Mixing Layer Thickness on Determination of Release Height Wind ................. 10

4 Chronological Sequence of Total Whole Body Dose

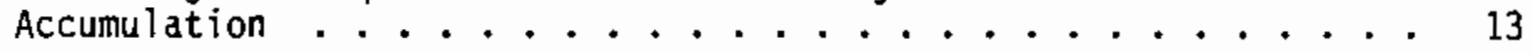

5 Dose Pathway Contributions to the Total Whole Body . . . . . 16

6 Internal Organ 50-year Dose Comnitments ........... 18

7 Residual Dose Rate at 00002 on May 7, 1986 . . . . . . . . 19

8 Gamna-Exposure Rate on May 29,1986 .............. 22

9 Oistribution of the Ratio of Observed Plume Centerline Concentrations to Values Predicted with MESOI .......... 27

10 Distribution of the Lateral Error Rate for MESOI Predictions of Plume Centerline Positions ............. 28

\section{$\underline{\text { TABLES }}$}

1 Radionuclide Source Terns Used in the MESORAD Dose Assessment ...................... 5

2 Radionuclides in the ORIGIN Core Inventory Not Included in the MESORAD Radionuclide Library............ 6

3 Meteorological Observation Locations ........... 8

4 Fraction of the MESORAD Domain with Various Accumulated

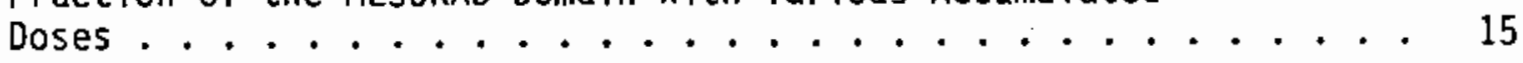

5 Area of the MESORAD Domain Within Various Residual Dose Rate Isopleths 


\section{INTRODUCTION}

Shortly after midnight local time on April 26, 1986, an accident involving Unit 4 of the Chernobylskaya Atomic Energy Station resulted in the release of a large amount of radioactive material to the atmosphere. The events leading to the accident are described in detail in other documents (USSR 1986, IAEA 1986, DOE 1987).

Briefly, power was being reduced on the 3200-Mht reactor, which had been in operation since December 1983, in preparation for a maintenance shutdown. while the reactor was at low power, an experiment was performed that resulted in ore or more prompt critical excursions at 1:23 a.m. local time on April 26, 1986 (23232 on April 25). These excursions led to an explosion, which destroyed the reactor, and fires that resulted in a protracted release of radioactive material to the atmosphere. Starting April 28, helicopters dropped materials on the reactor to control the release of radioactive effluents. By May 5 , 1986, the release was essentially complete.

Since the accident, a great deal of scientific attention has been focussed on the consequences of the release. Major areas of interest have included estimation of the health effects of the release based on radiation measurements and misdeling of the transport, diffusion, and deposition of the effluents from the accident on continental and global scales. This report describes an assessiment of the doses near the site (within $80 \mathrm{~km}$ ) prepared using the MESORAD dose issessment model (Scherpelz et al, 1986).

The MESORAD model, which was deveioped for emergency response applications for the U.S. Nuclear Regulatory Conmission and the U.S. Department of Energy, Richland Operations Office, combines relatively simple representations of the atmospheric processes of transport, diffusion, and deposition (Ramsdel1, Athey and Glantz 1983) with the computation of doses. The Chernobyl accident dose estimates are based on 27 radionuclides, taking decay and daughter product ingrowth and decay into account.

Tire assessment consists of dose and dose rate estimates at 961 nodes on a Cartisian grid. Chernobylskaya Unit 4 is located at the center of the grid, and the node spacing is approximately $5364 \mathrm{~m}$. The dose estimates generated by the model were: total whole body dose; contributions to the total whole 
body dose resulting from exposure to the airborne effluents, from exposure to material deposited on the ground, and from inhalation of the effluents; and doses to the lung and thyroid resulting from inhalation. In addition, whole body dose rates resulting from surface contamination were estimated. These estimates are depicted graphically.

In order, this report describes: 1) the source characteristics assumed for the releases from the Chernobyl accident, 2) the meteorological data and assumption used in the dose assessment, and 3) the results of the assessment. These sections are followed by a discussion of the results of the assessment and a discussion of the uncertainties associated with the MESORAD dose assessment. Appendices contain the meteorological data used in the assessment and results of the assessment in tabular form. 


\section{SOURCE CHARACTERIZATION}

Source characterization has two components. The first component is the specification of release locations and times, and the second component is the specification of the quantity of material released. None of the detajled reports covering the Chernobyl accident (USSR 1986, IAEA 1986, DOE 1987) provides a complete description of the physical conditions associated with the release. However, it is clear from these reports that the initial explosion carried effluents well above the surface, and that effluents released after the initial explosion had a lower effective release height.

For MESORAD model simulations of the Chernobyl accident, the release was divided into two parts. The first part was $2 \mathrm{~h}$ in duration. It included the initial explosive release. During this period, the source was assumed to be a vertical line extending from the reactor to an altitude of $1 \mathrm{~km}$. It was modeled by releasing puffs at altitudes of 200,500 , and $750 \mathrm{~m}$. The vertical distribution of release points during the first period is illustrated in Figure 1. The second part of the release was $238 \mathrm{~h}$ in duration. During this period, the source was modeled by releasing puffs at an altitude of $200 \mathrm{~m}$. All puffs were initially spherical with radii of approximately $30 \mathrm{~m}$.

"he radionuclide content of each of the puffs was postulated from an estimite of the radionuclide inventory in the core of the Chernobyl Unit 4 reactor at the time of the accident, an assumed release fraction for each radioruclide, and an assumed distribution of the release between the first and second release periods. The core inventory was estimated by an ORIGIN computer code simulation of an RBMK reactor. The ORIGIN inventory estimate is consistent with a Soviet estimate of the core inventory (DOE 1987). The release fraction and the distribution of release between periods assumed for each radionuclide were based on release fractions reported by the Soviets (USSR 1986, Annex 4 Table 4.14).

MESORAD dose computations only use radionuclides and dose factors contained in the MESORAD radionuclide library. Table 1 lists the radionuclides used in the ME:ORAD dose computations, the assumed core inventory, and the fraction releaseld in columns I through 3, respectively. The fourth column contains the friction of the release that was assumed to take place in the first period. 


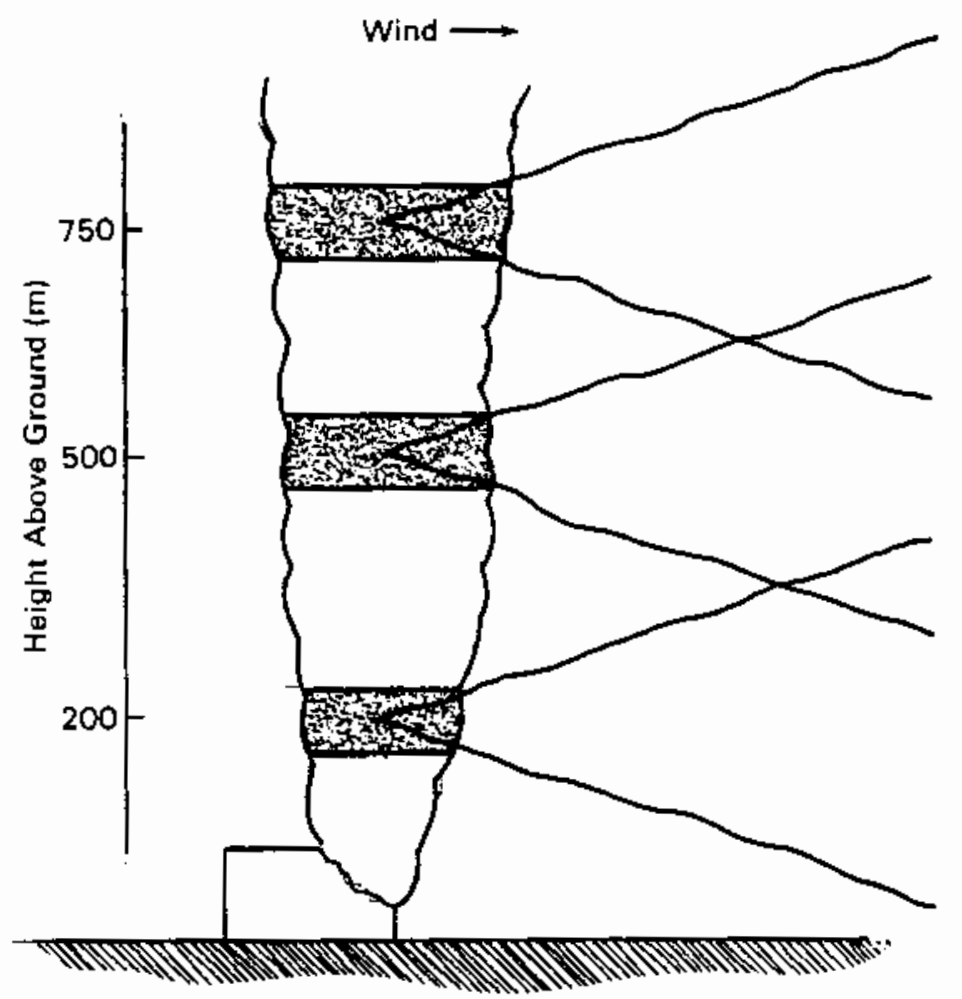

FIGURE 1. Representation of the Initial Release Configuration

During this period, $50 \%$ of the effluent was assumed to be released at $750 \mathrm{~m}$, and $30 \%$ was assumed to be released at $500 \mathrm{~m}$. The remaining $20 \%$ of the material was assumed to be released at $200 \mathrm{~m}$. Columns 5 through 7 give the total release for each radionuclide at each level during the first period, and column 8 gives the total release for each radionuclide for the second release period.

Not all of the radionuclides listed in the Soviet or ORIGIN core inventories are listed in the MESORAD radionuclide library. The radionuclides in the ORIGIN core inventory that are not in the MESORAD radionuclide library are listed in Table 2. It is likely that a small fraction of these radionuclides, perhaps 3 to $4 \%$, were released during the second release period. Some of these radionuclides are short-lived, and others do not undergo gamna decay. Therefore, neglecting these radionuclides resulted in underestimation of doses, but the amount of underestimation should not be large. 
IABLE 1. Radionuctide Source Terms Used in the MESORAD Dose Assessment

\begin{tabular}{|c|c|c|c|c|c|c|c|}
\hline \multirow[b]{2}{*}{$\begin{array}{l}\text { Radio- } \\
\text { nuclide }\end{array}$} & \multirow[b]{2}{*}{$\begin{array}{l}\text { Core Inv } \\
\text { (Ci) }\end{array}$} & \multirow[b]{2}{*}{ FRACTR } & \multirow[b]{2}{*}{ FRACT1 } & \multicolumn{4}{|c|}{ Iotal Release (Ci) } \\
\hline & & & & $\begin{array}{r}\text { Fir } \\
750 \mathrm{~m}\end{array}$ & $\frac{\text { Release }}{500 \mathrm{~m}}$ & $\frac{\text { riod }}{200 \mathrm{~m}}$ & $\begin{array}{l}\text { Second } \\
\text { Release } \\
\text { Period } \\
200 \mathrm{~m}\end{array}$ \\
\hline $\begin{array}{l}-85 \\
-85 M \\
-87 \\
-88 \\
-89 \\
-90 \\
-91 \\
91 \\
-163 \\
-1 C 6 \\
-129 \\
-129 M \\
-131 M \\
-132 \\
131 \\
132 \\
133 \\
134 \\
135 \\
-13.3 \\
-13 j \\
-13.1 \\
-131 \\
-13 . \\
140\end{array}$ & $\begin{array}{l}6.70 \mathrm{E}+05 \\
1.10 \mathrm{E}+07 \\
1.40 \mathrm{E}+07 \\
2.50 \mathrm{E}+07 \\
8.10 \mathrm{E}+07 \\
4.60 \mathrm{E}+06 \\
6.00 \mathrm{E}+07 \\
1.04 \mathrm{E}+08 \\
1.21 \mathrm{E}+08 \\
3.20 \mathrm{E}+07 \\
1.50 \mathrm{E}+07 \\
3.80 \mathrm{E}+06 \\
9.90 \mathrm{E}+06 \\
1.11 \mathrm{E}+08 \\
8.20 \mathrm{E}+07 \\
1.14 \mathrm{E}+08 \\
1.28 \mathrm{E}+08 \\
6.70 \mathrm{E}+07 \\
8.10 \mathrm{E}+07 \\
1.68 \mathrm{E}+08 \\
4.40 \mathrm{E}+07 \\
5.30 \mathrm{E}+06 \\
2.60 \mathrm{E}+06 \\
6.20 \mathrm{E}+06 \\
1.46 \mathrm{E}+08 \\
1.55 \mathrm{E}+08 \\
9.30 \mathrm{E}+07\end{array}$ & $\begin{array}{l}1.000 \\
1.000 \\
1.000 \\
1.000 \\
0.040 \\
0.040 \\
0.040 \\
0.040 \\
0.029 \\
0.029 \\
0.150 \\
0.150 \\
0.150 \\
0.150 \\
0.200 \\
0.200 \\
0.200 \\
0.200 \\
0.200 \\
1.000 \\
1.000 \\
0.100 \\
0.100 \\
0.130 \\
0.056 \\
0.056 \\
0.028\end{array}$ & $\begin{array}{l}0.2200 \\
0.2200 \\
0.2200 \\
0.2200 \\
0.0036 \\
0.0032 \\
0.0032 \\
0.0032 \\
0.0059 \\
0.0062 \\
0.0300 \\
0.0300 \\
0.0300 \\
0.0300 \\
0.1300 \\
0.1300 \\
0.1300 \\
0.1300 \\
0.1300 \\
0.1100 \\
0.1100 \\
0.0280 \\
0.0280 \\
0.0480 \\
0.0059 \\
0.0059 \\
0.0049\end{array}$ & $\begin{array}{l}7.37 \mathrm{E}+04 \\
1.21 \mathrm{E}+06 \\
1.54 \mathrm{E}+06 \\
2.75 \mathrm{E}+06 \\
1.46 \mathrm{E}+05 \\
7.36 \mathrm{E}+03 \\
9.60 \mathrm{E}+04 \\
1.66 \mathrm{E}+05 \\
3.57 \mathrm{E}+05 \\
9.92 \mathrm{E}+04 \\
2.25 \mathrm{E}+05 \\
5.70 \mathrm{E}+04 \\
1.49 \mathrm{E}+05 \\
1.67 \mathrm{E}+06 \\
5.33 \mathrm{E}+06 \\
7.41 \mathrm{E}+06 \\
8.32 \mathrm{E}+06 \\
4.36 \mathrm{E}+06 \\
5.27 \mathrm{E}+06 \\
9.24 \mathrm{E}+06 \\
2.42 \mathrm{E}+06 \\
7.42 \mathrm{E}+04 \\
3.64 \mathrm{E}+04 \\
1.49 \mathrm{E}+05 \\
4.31 \mathrm{E}+05 \\
4.57 \mathrm{E}+05 \\
2.28 \mathrm{E}+05\end{array}$ & $\begin{array}{l}4.42 \mathrm{E}+04 \\
7.26 \mathrm{0}+05 \\
9.24 \mathrm{E}+05 \\
1.65 \mathrm{E}+06 \\
8.75 \mathrm{E}+04 \\
4.42 \mathrm{E}+03 \\
5.76 \mathrm{E}+04 \\
9.98 \mathrm{E}+04 \\
2.14 \mathrm{E}+05 \\
5.95 \mathrm{E}+04 \\
1.35 \mathrm{E}+05 \\
3.42 \mathrm{E}+04 \\
8.91 \mathrm{E}+04 \\
9.99 \mathrm{E}+05 \\
3.20 \mathrm{E}+06 \\
4.45 \mathrm{E}+06 \\
4.99 \mathrm{E}+06 \\
2.61 \mathrm{E}+06 \\
3.16 \mathrm{E}+06 \\
5.54 \mathrm{E}+06 \\
1.45 \mathrm{E}+06 \\
4.45 \mathrm{E}+04 \\
2.18 \mathrm{E}+04 \\
8.93 \mathrm{E}+04 \\
2.58 \mathrm{E}+05 \\
2.74 \mathrm{E}+05 \\
1.37 \mathrm{E}+05\end{array}$ & $\begin{array}{l}2.95 \mathrm{E}+04 \\
4.84 \mathrm{E}+05 \\
6.16 \mathrm{E}+05 \\
1.10 \mathrm{E}+06 \\
5.83 \mathrm{E}+04 \\
2.94 \mathrm{E}+03 \\
3.84 \mathrm{E}+04 \\
6.66 \mathrm{E}+04 \\
1.43 \mathrm{E}+05 \\
3.97 \mathrm{E}+04 \\
9.00 \mathrm{E}+04 \\
2.28 \mathrm{E}+04 \\
5.94 \mathrm{E}+04 \\
6.66 \mathrm{E}+05 \\
2.13 \mathrm{E}+06 \\
2.96 \mathrm{E}+06 \\
3.33 \mathrm{E}+06 \\
1.74 \mathrm{E}+06 \\
2.11 \mathrm{E}+06 \\
3.70 \mathrm{E}+06 \\
9.68 \mathrm{E}+05 \\
2.97 \mathrm{E}+04 \\
1.46 \mathrm{E}+04 \\
5.95 \mathrm{E}+04 \\
1.72 \mathrm{E}+05 \\
1.83 \mathrm{E}+05 \\
9.11 \mathrm{E}+04\end{array}$ & $\begin{array}{l}5.23 \mathrm{E}+05 \\
8.58 \mathrm{E}+06 \\
1.09 \mathrm{E}+07 \\
1.95 \mathrm{E}+07 \\
2.95 \mathrm{E}+06 \\
1.69 \mathrm{E}+05 \\
2.21 \mathrm{E}+06 \\
3.83 \mathrm{E}+06 \\
2.80 \mathrm{E}+06 \\
7.30 \mathrm{E}+05 \\
1.80 \mathrm{E}+06 \\
4.56 \mathrm{E}+05 \\
1.19 \mathrm{E}+06 \\
1.33 \mathrm{E}+07 \\
5.74 \mathrm{E}+06 \\
7.98 \mathrm{E}+06 \\
8.96 \mathrm{E}+06 \\
4.69 \mathrm{E}+06 \\
5.67 \mathrm{E}+06 \\
1.50 \mathrm{E}+08 \\
3.92 \mathrm{E}+07 \\
3.82 \mathrm{E}+05 \\
1.87 \mathrm{E}+05 \\
5.08 \mathrm{E}+05 \\
7.31 \mathrm{E}+06 \\
7.77 \mathrm{E}+06 \\
2.15 \mathrm{E}+06\end{array}$ \\
\hline
\end{tabular}

FRACTF = Fraction of core inventory released FRACTI = Fraction of core inventory released in first $2 \mathrm{~h}$ $M=$ Metastable 
TABLE 2. Radionuclides in ORIGIN Core Inventory Not Included in the MESORAD Radionuclide Library

\begin{tabular}{|c|c|}
\hline Radionuclide & Core Inventory \\
\hline $\begin{array}{l}\text { Y }-90 \\
\text { Zr-95 } \\
\text { Zr- } 97 \\
\text { Nb- } 95 \\
\text { Mo-99 } \\
\text { Tc-99 } \\
\text { Ru-105 } \\
\text { Rh-105 } \\
\text { Ce-141 } \\
\text { Ce-143 } \\
\text { Pr-143 } \\
\text { Nd-147 } \\
\text { Pm- } 147 \\
\text { Pm-149 }\end{array}$ & $\begin{array}{l}4.70 E+06 \\
1.38 E+08 \\
9.80 E+07 \\
1.37 E+08 \\
1.40 E+08 \\
1.28 E+08 \\
3.80 E+07 \\
6.50 E+07 \\
1.38 E+08 \\
1.08 E+08 \\
1.28 E+08 \\
5.50 E+08 \\
1.10 E+07 \\
3.10 E+07\end{array}$ \\
\hline
\end{tabular}




\section{METEOROLOGICAL CONDITIONS}

At the time of the accident, the Chernobyl area was under the influence of a weak high pressure system. Winds near ground level were light and variable. Under these conditions winds at the reactor site would be expected to display the effects of local topographic features. At an altitude of about $1500 \mathrm{~m}(850 \mathrm{mb})$, the wind was out of the east-southeast at 8 to $10 \mathrm{~m} / \mathrm{s}$. High pressure and low winds continued throughout April 26 and 27. On April 28, the upper-level winds shifted to the south in the morning and to the westsouthwest in the afternoon. During this period the surface winds remained light and variable. By late afternoon on April 29, the upper-level wind had shifted to the north. On May 1, the surface winds came around to the north, and the speeds at both the surface and upper level increased. The northerly winds persisted for the rest of the release period. Thus, based on the winds in the lower layer of the atmosphere, effluents from the accident initially contaminated the area to the west of reactor. As the wind shifted, the area of contamination spread to the north and finally to the south.

No appreciable precipitation was reported in the Chernobyl area during the entire 10-day release period. Therefore, the surface contamination that occurred was the result of relatively slow processes taking place near the surface. Had precipitation occurred, the surface contamination would have been significantly greater.

Meteorological data required for MESORAD simulations include wind speed and direction at ground level, upper-level wind speed and direction, an indicator of atmospheric stability, and an estimate of the thickness of the atmospheric mixing layer. The meteorological data for use in the MESORAD dose assessment for the Chernobyl releases were available for a location near Chernobyl and for eight rather distant locations. These locations are shown in Figure 2, and coordinates for the observations are given in Table 3. Groundlevel wind data (assumed to be measured at $10 \mathrm{~m}$ ) were available for all locations; upper-level data were available for Kiev and Gomel. Unfortunately, the data for the location near Chernobyl were not available for the entire 


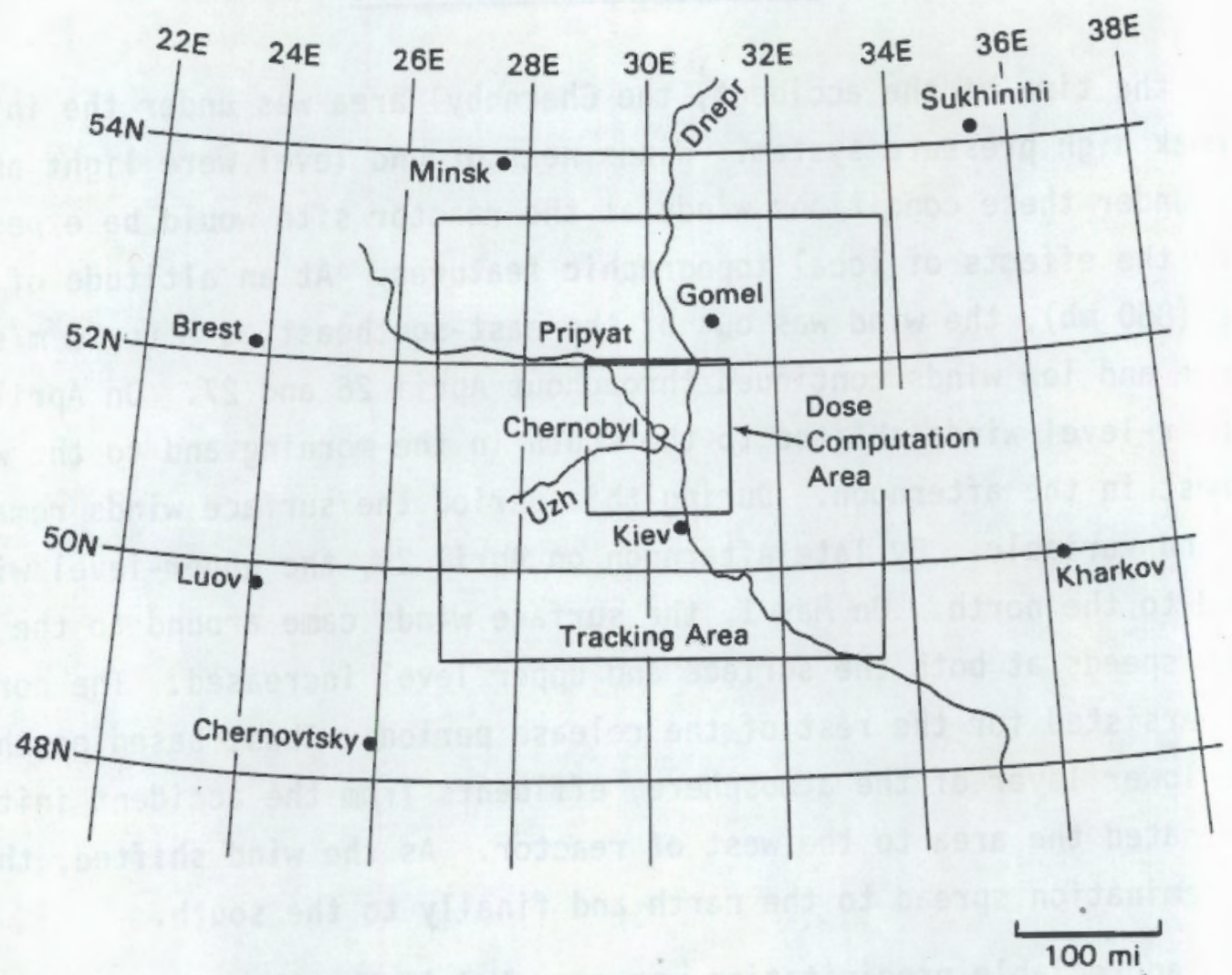

FIGURE 2. MESORAO Model Domain and Meteorological Observation Locations

TABLE 3. Meteorological Observation Locations

\begin{tabular}{|c|c|c|c|}
\hline Location & $\begin{array}{c}\text { Appendix A } \\
\text { Abbreviation }\end{array}$ & Latitude & Longitude \\
\hline Chernobyl & CHNBYL & $\begin{array}{l}51^{\circ} 17^{\prime} N \\
51^{\circ} 24^{\prime} N(a)\end{array}$ & $\begin{array}{l}30^{\circ} 14^{\prime} E \\
30^{\circ} 05^{\prime} E(a)\end{array}$ \\
\hline Kiev & $\begin{array}{l}\text { KIEV } \\
\text { GOMEI }\end{array}$ & $50^{\circ} 24^{\prime N}$ & $30^{\circ} 27^{\prime} \mathrm{E}$ \\
\hline $\begin{array}{l}\text { Gome } 1 \\
\text { Minsk }\end{array}$ & GOMEL & $52^{\circ} 27^{\prime} \mathrm{N}$ & $31^{\circ} 00^{\prime} \mathrm{E}$ \\
\hline $\begin{array}{l}\text { Minsk } \\
\text { Sukhinihi }\end{array}$ & $\begin{array}{l}\text { MINSK } \\
\text { SUKHIN }\end{array}$ & $\begin{array}{l}53^{\circ} 52^{\prime} \mathrm{N} \\
54^{\circ} 07^{\prime} \mathrm{N}\end{array}$ & $\begin{array}{l}27^{\circ} 32^{\prime} \mathrm{E} \\
35^{\circ} 20^{\prime} \mathrm{E}\end{array}$ \\
\hline Brest & BREST & $52^{\circ} 07^{\circ} \mathrm{N}$ & $23^{\circ} 41^{\prime} \mathrm{E}$ \\
\hline Lvov & LVOV & $49^{\circ} 49^{\prime} \mathrm{N}$ & $23^{\circ} 57^{\prime} \bar{E}$ \\
\hline Chernovtsy & CHRNOV & $48^{\circ} 16^{\prime} \mathrm{N}$ & $25^{\circ} 58^{\prime} \mathrm{E}$ \\
\hline Kharkov & KHARKV & $49^{\circ} 56^{\prime} \mathrm{N}$ & $36^{\circ} 17^{\prime} \mathrm{E}$ \\
\hline
\end{tabular}

(a) Assumed location of the observation. 
release period. The temporal resolution of the ground-level data is $3 \mathrm{~h}$ and that of the upper-level data is $6 \mathrm{~h}$. The complete set of meteorological data used as input to MESORAD is listed in Appendix A.

The MESORAD mode1 domain is shown in Figure 2 . The inner box of the model domain contains the region for which dose estimates were made, and the outer box shows a region in which puffs were tracked without computing doses.

The winds at the release height are used in estimating puff movement. These winds are computed from the surface wind field, which is derived from the available data and the upper-level wind. The surface wind field is defined at grid nodes within the dose computation area using an interpolation scheme in which the observed data are weighted by the inverse of the square of the distance between the node and the measurement location. Thus, although ground-level wind data are available for nine locations, winds at Chernobyl, Kiev, and Gomel dominate the winds for other locations in definition of the surface wind field.

The release height winds are estimated by linear interpolation between the north-south and east-west wind components in the surface wind field and the corresponding components of the upper-level wind. For purposes of estimating the release height wind, the upper-level wind is assumed to be representative of the wind at the top of mixing layer. When the mixing layer is relatively thin, as it is at night, the release height wind direction and speed are near the direction and speed of the upper-level wind. Similarly, when the mixing layer is relatively thick, as it is during the day, the release height wind tends to be close to the surface wind. This relationship is illustrated in Figure 3 for a release height of $200 \mathrm{~m}$. 

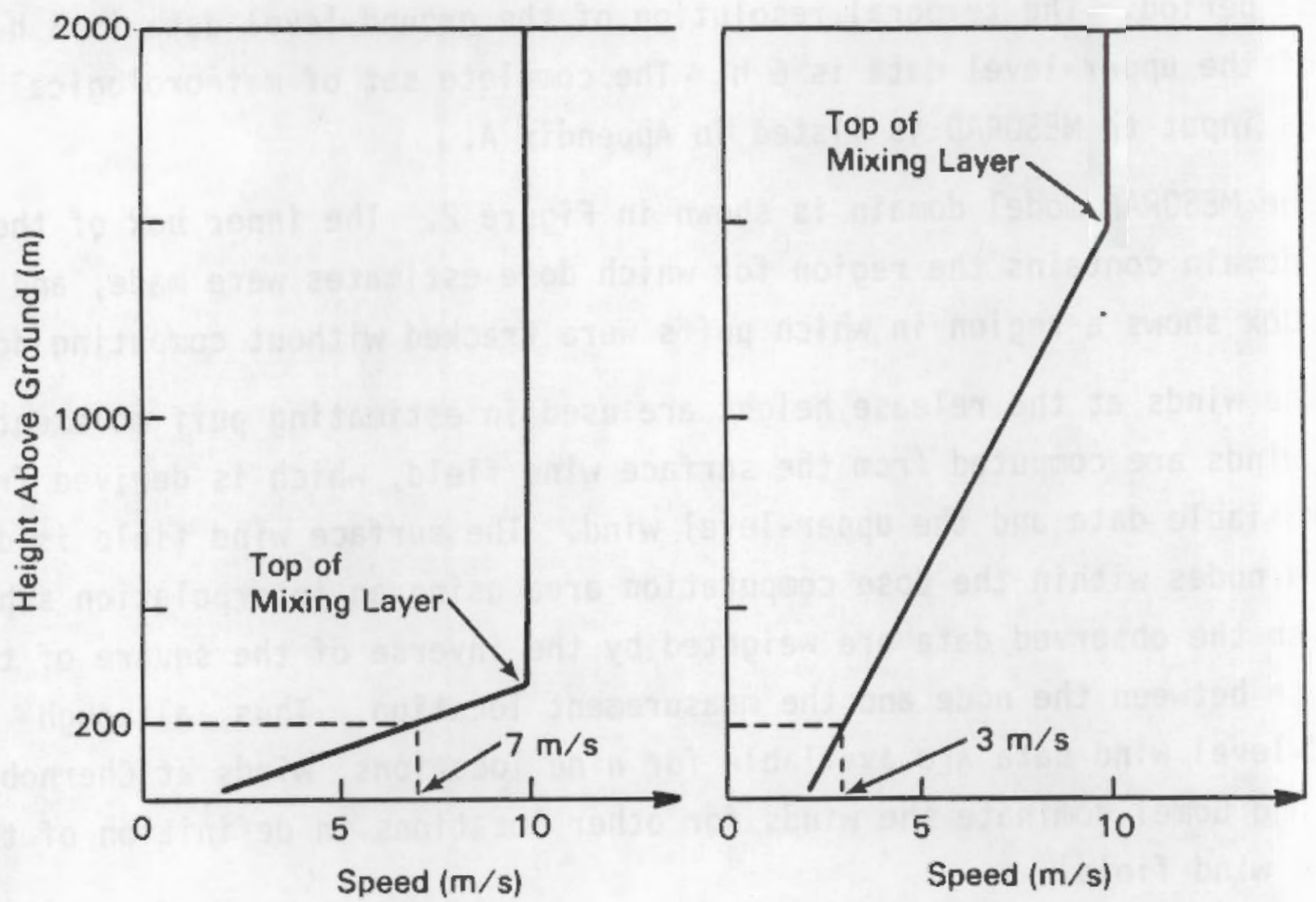

FIGURE 3. Effect of Mixing Layer Thickness on Determination of Release Height Wind 


\section{DOSE ASSESSMENT}

MESORAD estimates doses to the whole body and to the thyroid and lung via three pathways. These pathways are

1) external radiation from airborne radionuclides

2) external radiation from radionuclides deposited on the ground

3) internal radiation from inhaled radionuclides.

The doses are estimated from dose rates computed each $15 \mathrm{~min}$ at each receptor. A11 doses and dose rates are based on the assumption that the receptor remains in place from the beginning of the simulation to the end. Doses to receptors that move during the simulation period--people, for example--will be different than those computed by MESORAD.

The external dose from airborne radionuclides is estimated using semiinfinite and finite cloud approximations as appropriate. The finite cloud approximation is used for dose estimates near the source. It considers the spatial distribution of the radionuclides in the plume, the distance between the plume and the receptor, and range of gamma rays in air in the dose estimates. The finite cloud approximation provides good dose estimates at all distances but is too time-consuming to be used at all distances. The semiinfinite approximation is used when the plume becomes widely dispersed. It uses the concentration of radionuclides in air at the receptor location in estimating doses. The semi-infinite cloud approximation provides good dose estimates when radionuclide are widely dispersed.

The external dose from radionuclides deposited on the ground is estimated by assuming that the ground is an infinite flat plane and that the surface contamination $\left(\mathrm{Ci} / \mathrm{m}^{2}\right)$ everywhere is the same as the contamination at the receptor location. Surface contamination is estimated in MESORAD using both wet and dry deposition models. No appreciable precipitation occurred at Chernobyl between April 26 and May 7; therefore, all surface contamination is assumed to have been the result of dry deposition.

The dry deposition model used in MESORAD assumes that the rate of deposition for each radionuclide is proportional to its concentration near the surface. The constant of proportionality has the units of speed and is called the deposition velocity. For the Chernobyl accident, a deposition velocity 
of $0.01 \mathrm{~m} / \mathrm{s}$ was assumed for all isotopes of iodine, and for all the depositing radionuclides a deposition velocity of $0.001 \mathrm{~m} / \mathrm{s}$ was assumed. Noble gases were assumed not to deposit. In estimating doses from surface contamination, neither any shielding by obstacles nor weathering of the contamination has been considered. Similarly, the surface contamination dose estimates do not take into account the effects of radiation from material that was deposited near the reactor because it had an appreciable settling velocity.

The dose estimated for the inhalation pathway is a 50 -year dose commitment. It includes the immediate dose and a dose projection based on the body's retention of the radionuclides. The inhalation dose commitment at each receptor is directly proportional to the airborne concentration of each radionuclide. Inhalation pathway dose commitment estimates have been made for the whole body and for the adult thyroid and lung.

The chronological sequence in the expansion of the local area affected by radiation from the Chernobyl accident is shown in frames (a) through ( $h$ ) of Figure 4. The first frame (a) shows the total whole body dose pattern estimated by MESORAD at $0000 Z$ on April 27. This pattern is based on the sum of the doses estimated for the external exposure pathways and the 50-year whole body dose commitment for the inhalation pathway. The relatively narrow band shown indicates the steady nature of the meteorological conditions during the first few hours of the release. Frames (b) through $(g)$ show the expansion of the pattern in 24-h intervals over the next 6 days. The shift in wind directions from easterly to southeasterly and then to southwestly is clearly evident in frames (a), (b), and (c). On April 29, the winds, although light, shifted to northwesterly resulting in a slow drift of the dose pattern to the south that continued for the next several days. Frames (d) through $(\mathrm{g})$ show this expansion. Frame (h) shows dose estimates for $0000 \mathrm{Z}$ on May 7, approximately 1 day after the time that releases from the reactor were assumed to have ended. Although doses would have continued to increase with time as a result of surface contamination, the basic dose pattern shown in frame ( $h$ ) would have remained unchanged if the MESORAD simulation had continued beyond $0000 Z$ on May 7. The model receptor with the maximum total whole body dose at this time is located $4.7 \mathrm{mi}$ northwest of the reactor. The accumulated dose at this location was 9.9 rem. 

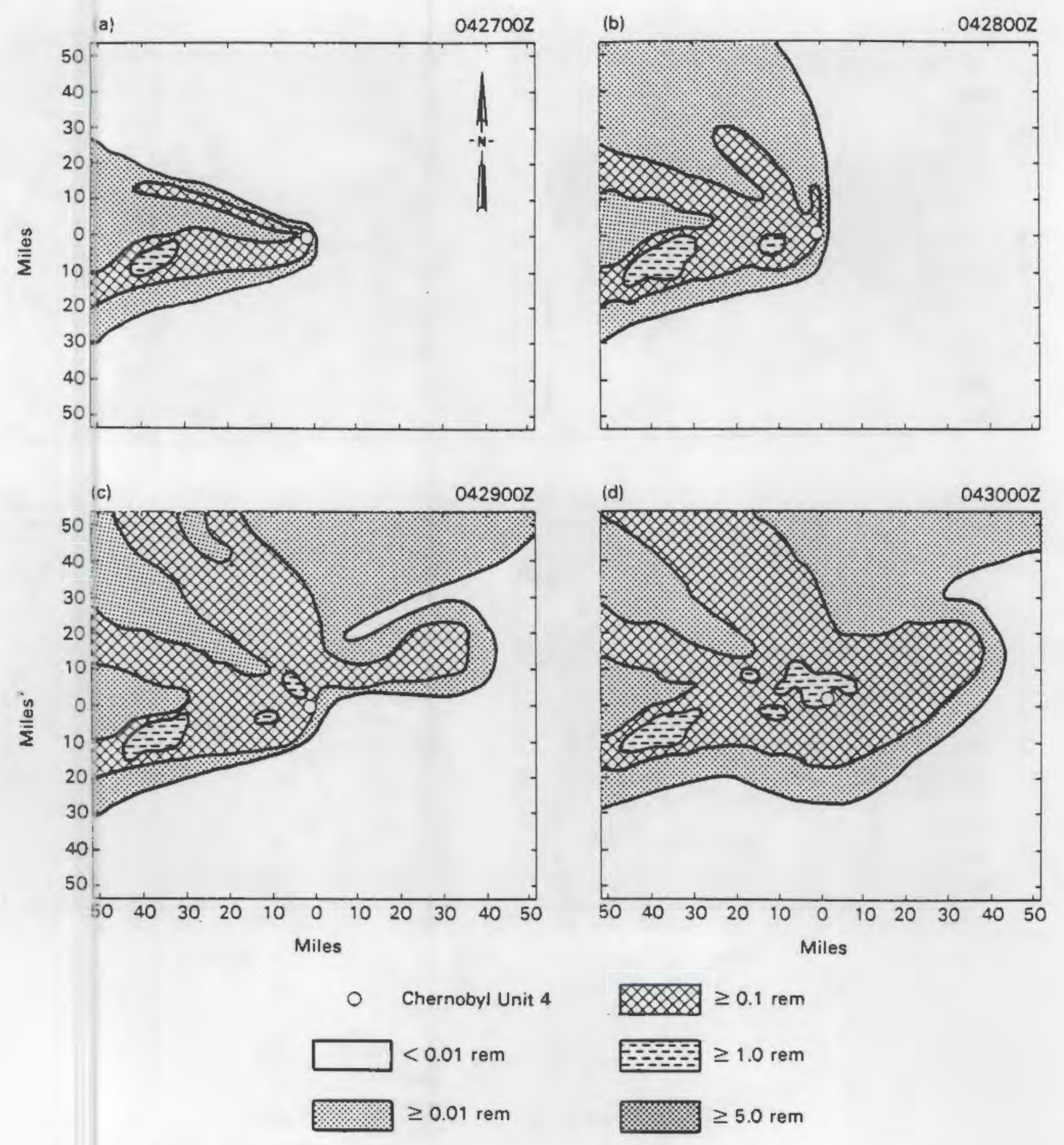

FIGURE 4. Chronological Sequence of Total Whole Body Dose Accumulation 

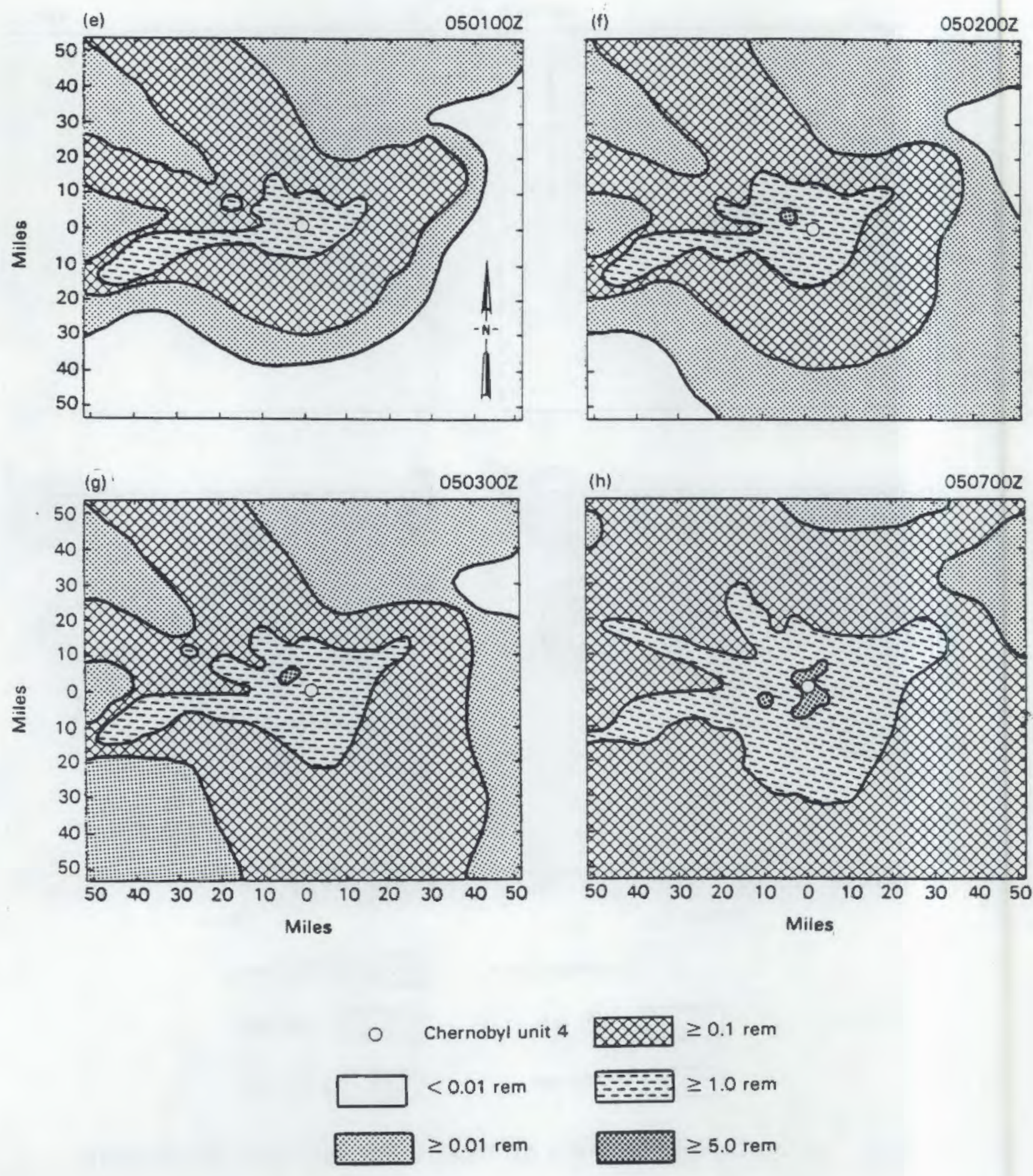

FIGURE 4. (contd) 
The total area exceeding various doses can be estimated by assuming that each receptor within the MESORAD model domain represents an area of $11.11 \mathrm{mi}^{2}$, which is equal to the square of the separation between receptors. With this assumption, the total area within the model domain is $10678 \mathrm{mi}^{2}$. Table 4 gives the fraction of this area in which doses exceeded 1,2, and $5 \mathrm{rem}$ as a function of time during the course of the release period. By 00002 on May 7 , approximately $21.4 \%$ of the land within the model domain had accumulated a whole body dose of 1 rem or more, and about $1.5 \%$ of the land had accumulated a dose of 5 rem or more. On May 7, the fractions of the total area with accumulated doses equaling or exceeding $0.5,0.2$, and 0.1 rem were 0.409 , 0.767 , and 0.930 , respectively. In estimating the areas on which the fractions are based, the dose at the receptor at the source was assumed to be equal to or greater than the highest dose at any other receptor. The estimates of area fractions enclosed within the higher dose isopleths have a greater uncertainty than the fractions associated with the lower dose isopleths because of limited model resolution near the release point.

The contribution of each of the pathways to the total whole body dose estimates for $0000 z$ on May 7 , shown in frame (h) of Figure 4, is shown in Figure 5. The first frame shows the dose resulting from the airborne plume; the second frame shows the dose commitment from inhalation, and the last frame

TABLE 4. Fraction of the MESORAD Domain with Various Accumulated Doses. The area estimates are as of $0000 z$ on the given date.

\begin{tabular}{llcc} 
& \multicolumn{3}{c}{ Dose (rem) } \\
\cline { 2 - 3 } Date & 1 & 2 & 5 \\
\cline { 2 - 3 } $4 / 27$ & 0.0073 & & \\
$4 / 28$ & 0.0146 & 0.0021 & \\
$4 / 29$ & 0.0166 & 0.0083 & \\
$4 / 30$ & 0.0281 & 0.0094 & \\
$5 / 1$ & 0.0562 & 0.0166 & 0.0010 \\
$5 / 2$ & 0.0874 & 0.0260 & 0.0021 \\
$5 / 3$ & 0.110 & 0.0343 & 0.0021 \\
$5 / 4$ & 0.132 & 0.0479 & 0.0021 \\
$5 / 5$ & 0.159 & 0.0614 & 0.0073 \\
$5 / 6$ & 0.194 & 0.0822 & 0.0114 \\
$5 / 7$ & 0.214 & 0.0937 & 0.0146
\end{tabular}



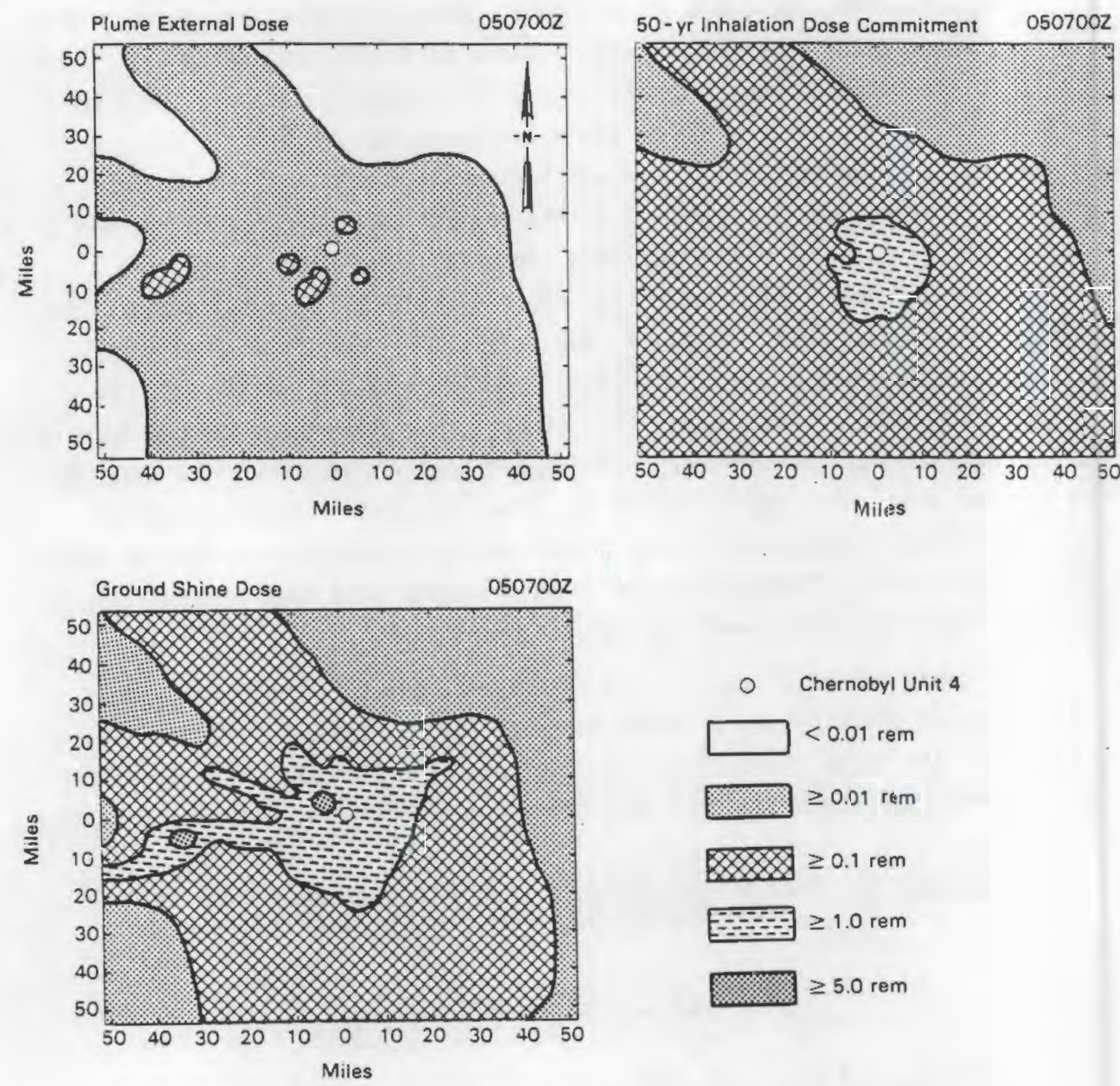

FIGURE 5. Dose Pathway Contributions to the Total Whole Body 
shows the dose from surface contamination. Comparing the doses shown in the three frames shows that the most significant contribution to the total whole body dose is from surface contamination. The inhalation dose comnitment is significant, but less important than the dose from surface contamination. The external dose resulting from the airborne plume is seen to make an insignificant-contribution to the total. Unfortunately, the estimated doses from surface contamination have greater uncertainty than doses estimated for either of the other two pathways.

The receptor with the maximum dose from the airborne plume is located 41.4 mi west-southwest of the reactor. It is likely that much of the dose of 0.14 rem in this region is the result of passage of the initial plume. The location of the highest ground-shine contribution to whole body dose ( $7.4 \mathrm{rem}$ ) is $4.7 \mathrm{mi}$ northwest, at the same location as the highest total whole body dose. The maximum inhalation whole body dose commitment $(2.4$ rem) occurs at two locations: $4.7 \mathrm{mi}$ northwest and $7.5 \mathrm{mi}$ south-southwest of the reactor.

The 50-year dose commitments to the adult thyroid and lung are shown in Figure 6 . The thyroid dose pattern (the upper frame in Figure 6) shows a band of significant exposure to the west-southwest of Chernobyl. This band results from the large early release of iodine. The maximum thyroid dose commitment ( $98 \mathrm{rem}$ ) occurs in this band, about $41.2 \mathrm{mi}$ from the reactor. The dose commitment pattern for the lung, shown in the lower frame, resembles the total whole body dose pattern shown in frame $(h)$ of Figure 4. Maximum lung dose commitments of 93 rem are estimated to have occurred $4.7 \mathrm{mi}$ northwest and $7.5 \mathrm{mi}$ south-southwest of the reactor.

At the end of the MESORAD simulation (0000Z on May 7$)$, no airborne material from the Chernobyl releases remained within the dose computation portion of the model domain. As a result, barring further releases from the reactor, the only pathway that would contribute to additional exposure at the receptors is the surface contamination pathway. The dose rate for this pathway at the end of the simulation is shown in Figure 7. The area and fraction of the model domain within the $1,2,5,10$, and $20 \mathrm{mrem} / \mathrm{h}$ isopleths are 1 isted in Table 5. The receptor in the MESORAD model domain with the maximum dose rate is located $7.5 \mathrm{mi}$ south-southwest of the reactor. The May 7, $0000 \mathrm{z}$ dose rate 

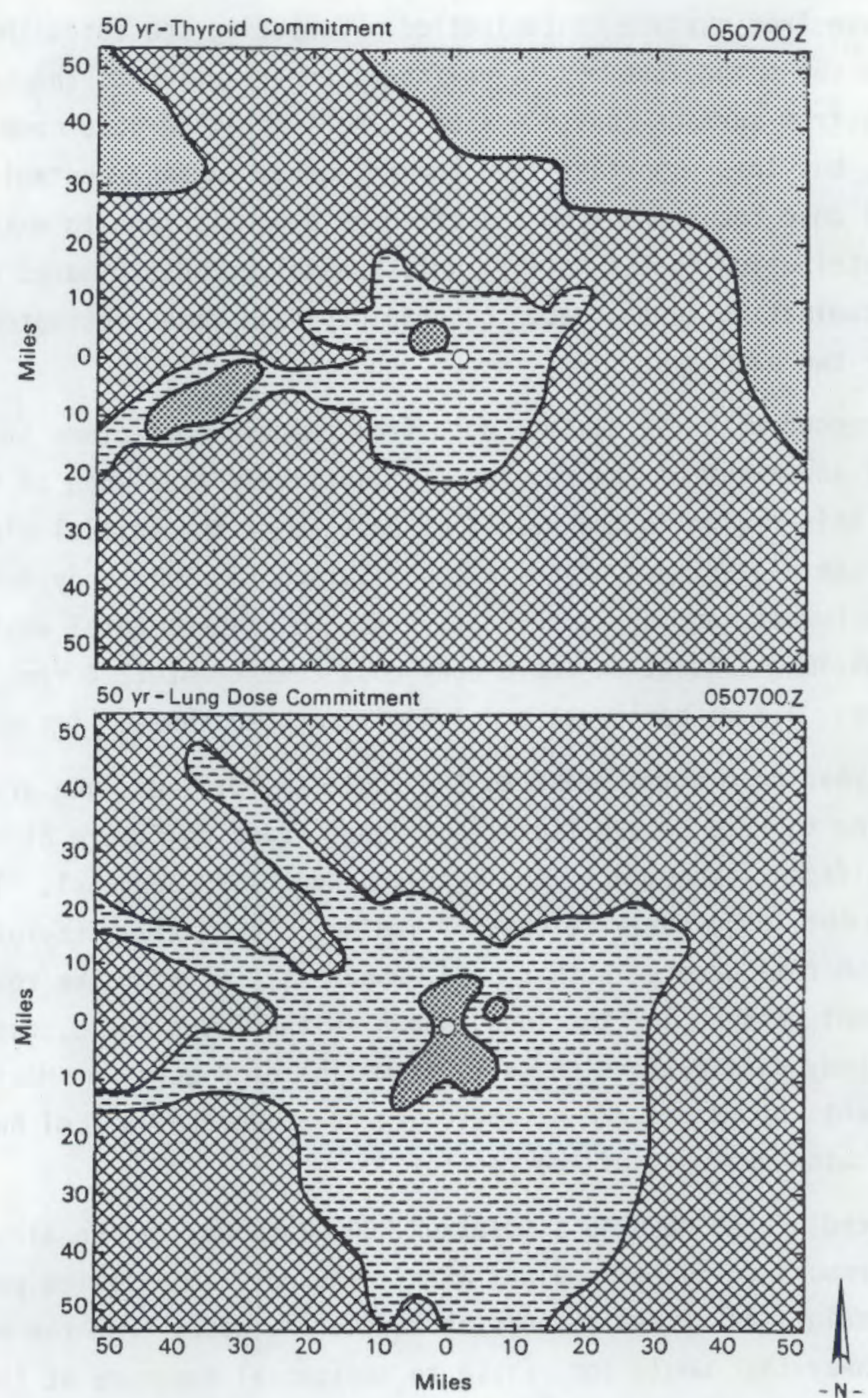

O Chernobyl Unit 4

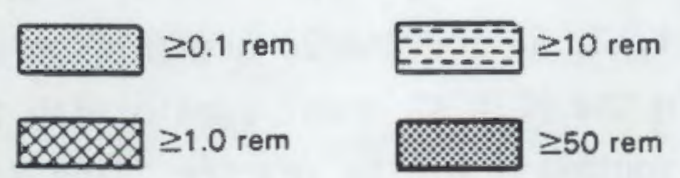

FIGURE 6. Internal Organ 50-year Dose Commitments 


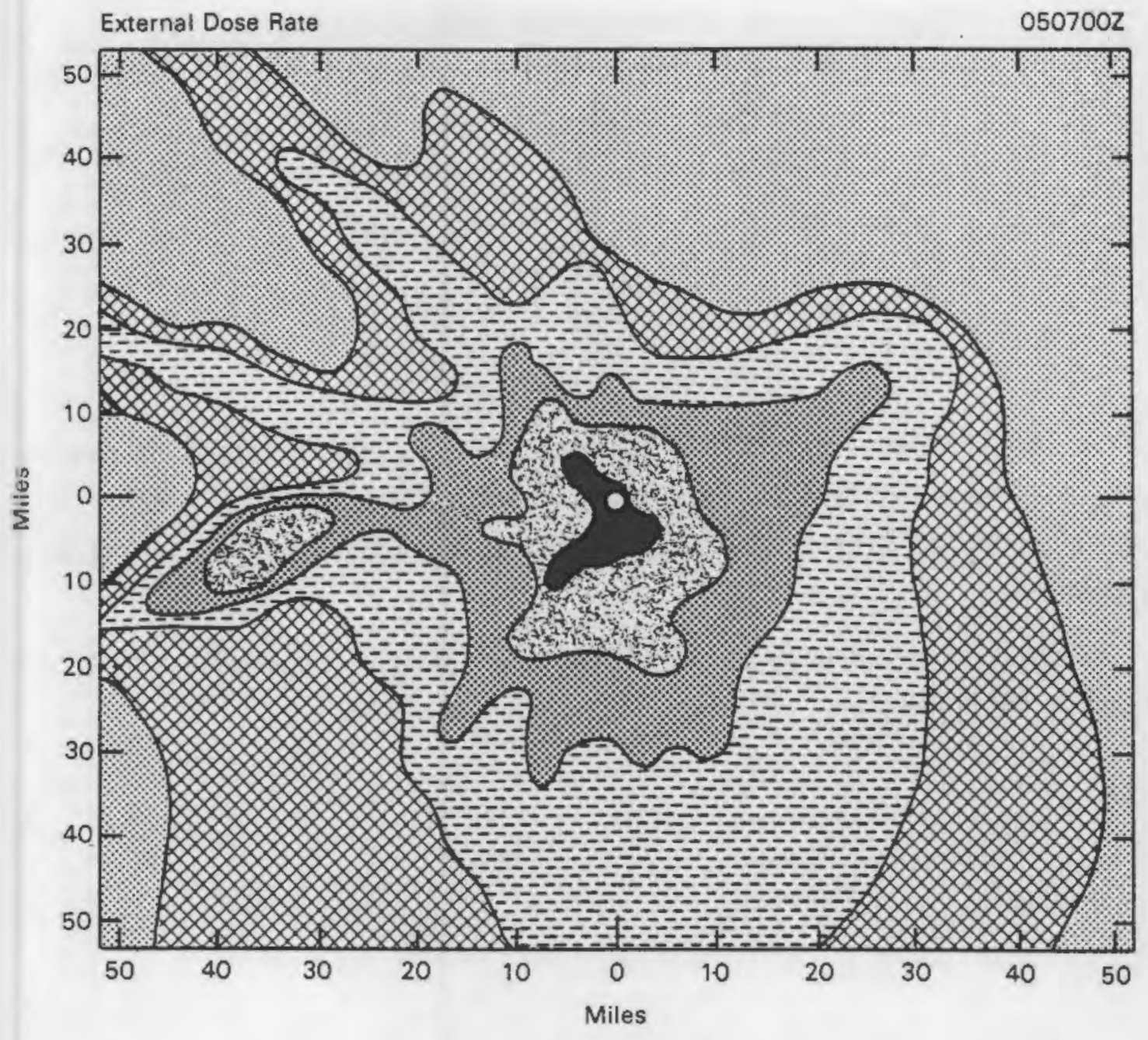

- Chernobyl unit 4

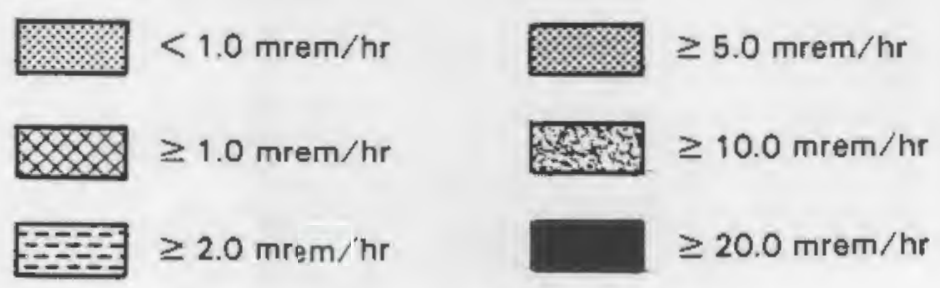

FIGURE 7. Residual Dose Rate at 0000 z on May 7, 1986 
TABLE 5. Area of the MESORAD Domain Within Various Dose Rate Isopleths

\begin{tabular}{|c|c|c|}
\hline $\begin{array}{l}\text { Dose Rate } \\
\text { (mrem } / \mathrm{h})\end{array}$ & $\begin{array}{l}\text { Area } \\
(m i 2) \\
\end{array}$ & $\begin{array}{l}\text { Model Domain } \\
\text { Fraction }\end{array}$ \\
\hline $\begin{array}{l}>20 \\
>10 \\
>5 \\
>2 \\
>1\end{array}$ & $\begin{array}{r}78 \\
611 \\
1630 \\
4490 \\
7440\end{array}$ & $\begin{array}{l}0.0073 \\
0.0572 \\
0.153 \\
0.420 \\
0.697\end{array}$ \\
\hline
\end{tabular}

at this location was $39 \mathrm{mrem} / \mathrm{h}$. The dose rate at the location of maximum accumulated whole body dose (4.7 $\mathrm{mi}$ to the northwest of the reactor) was $34 \mathrm{mrem} / \mathrm{h}$. It is likely that there are locations with higher dose rates near the reactor.

MESORAD dose and dose rate predictions for $0000 Z$ on May 7, 1986, are presented in Appendix B. 


\section{DISCUSSION}

MESORAD is a dose model used in emergency response applications by the U.S. Department of Energy at its Hanford Site in Washington State and in the U.S. Nuclear Regulatory Commission Operations Center in Bethesda, Maryland. The model has been developed on the basis of generally accepted dispersion theory and uses diffusion and deposition parameterizations that are based on extensive sets of experimental data. The diffusion and transport portions of the model have been evaluated using experimental data. However, the full dose-assessment model has never been validated. Further, in typical applications, model simulations are made using a more extensive meteorological data base than is available for the Chernobyl accident. As a result, comparison of the results of the MESORAD simulation with observed data from Chernobyl is of interest.

The Chernobyl data that are most comparable with MESORAD simulation results are the gamma radiation levels shown in Figure 5.4 of the Soviet report (USSR 1986). This figure, which is also Figure 5.2 of the U.S. Department of Energy report on the Chernobyl accident (DOE 1987), is reproduced in Figure 8 . The dose rates from which the pattern was drawn were observed on May 29, approximately 3 weeks following the end of the MESORAD simulation. In Figure 8 , the release point is just west of the town of Pripyat. It should be noted that some of the isopleths in Figure 8 are mislabelled.

Before Figures 7 and 8 can be compared, it is necessary to correct the dose rates given in Figure 7 for decay, and convert the units of mrem $/ \mathrm{h}$ in Figure 7 to $\mathrm{mR} / \mathrm{h}$ shown in Figure 8. If we assume that the residual dose rates shown in Figure 7 are primarily due to $131 \mathrm{I}$ and $137 \mathrm{Cs}$, decay from $0000 \mathrm{Z}$ on May 7 to $1200 Z$ on May 29 would decrease dose rates by about $50 \%$. The actual decrease in dose rates is a function of the isotopic mix at each location and varies from about 40 to $60 \%$ within the MESORAD domain. A gamma exposure rate of $1 \mathrm{mR} / \mathrm{h}$ is approximately equal to a dose rate $0.9 \mathrm{mrem} / \mathrm{h}$. Thus, for qualitative comparisons, the $2 \mathrm{mrem} / \mathrm{h}$ dose-rate isopleth in Figure 7 is approximately equivalent to the $1 \mathrm{mR} / \mathrm{h}$ gamma-exposure-rate isopleth in Figure 8 . 


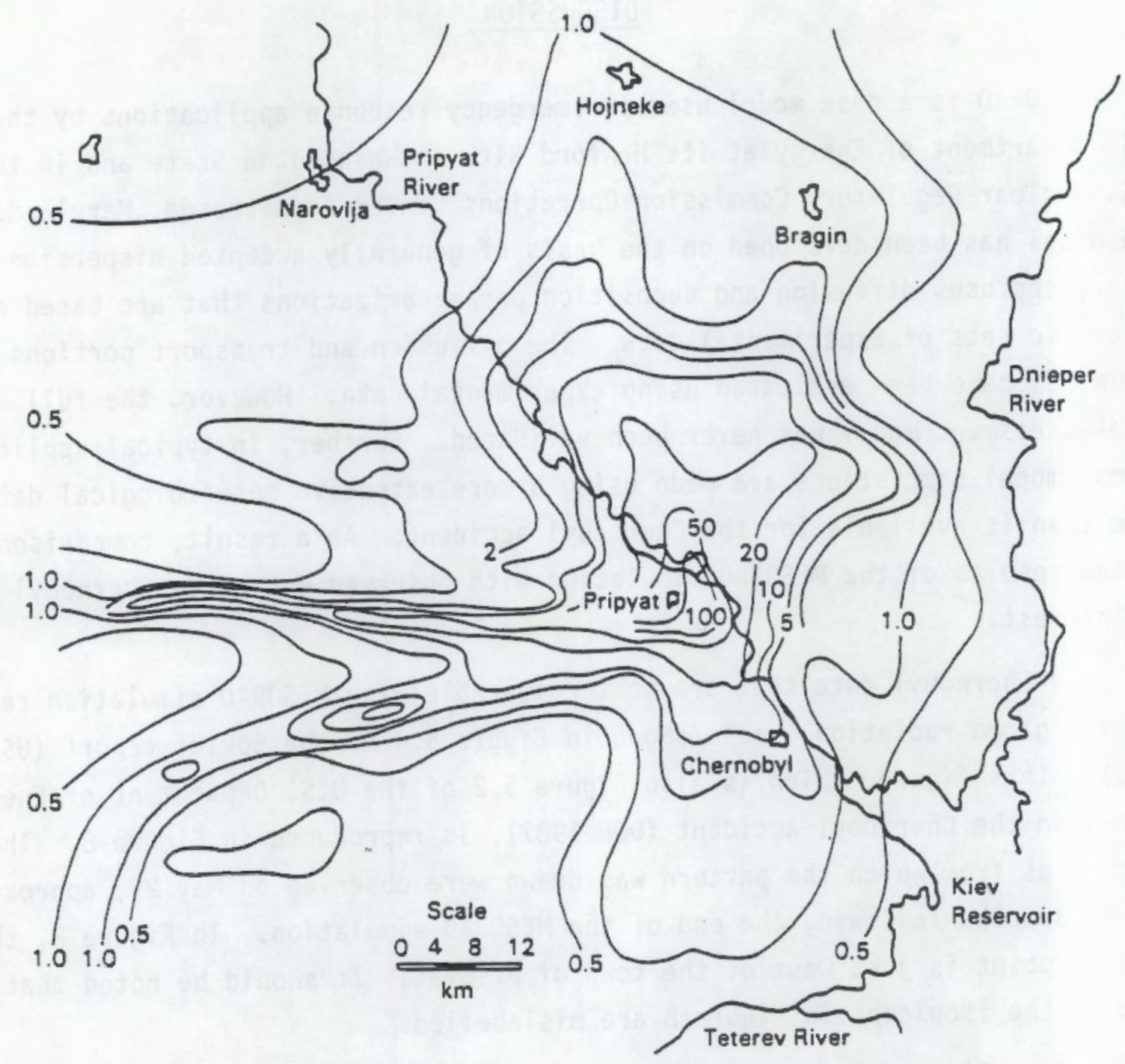

FIGURE 8. Gamma-Exposure Rate (mR/h) on May 29, 1986

The agreement of the dose rate patterns in these figures indicates that most of the assumptions entering into the MESORAD computation were reasonable. However, in two areas the patterns were sufficiently different to warrant further discussion. These regions are the area directly west of the Chernobyl reactor and the area east of the Dnieper River.

It is likely that the band of relatively high dose rates west of the reactor site was caused by fallout of particles from the initial release of material. If this is a correct assumption, the position of the band indicates that the wind direction controlling the initial transport of material was 
about $272^{\circ}$. To obtain this initial transport direction, the upper-level wind direction used in the MESORAD simulation for the first $6 \mathrm{~h}$ of the release was changed to $090^{\circ}$ from $110^{\circ}$, which was reported for the $850-\mathrm{mb}$ level at Kiev. This was the only change that was made in the reported meteorological data, although several values appeared questionable.

The differences in magnitude of the dose rates in the high dose-rate band to the west of Chernobyl may be the result of processes not modeled by MESORAD. MESORAD does not treat the transport, diffusion, and deposition of large particles. These particles have large gravitational settling rates that bring them to the ground much sooner than is accounted for by normal diffusion.

The observed dose-rate pattern in Figure 8 does not indicate that any material was deposited east of the Dnieper River east of Chernobyl, although the MESORAD dose-rate pattern predicts measurable dose rates in this area. Possible causes of this difference include inadequate representation of the wind field and incorrect estimation of the release rate during westerly winds.

The portion of the release responsible for the predicted deposition east of the Dnieper River occurred between $1700 Z$ on April 28 and $0100 Z$ on April 29 with approximately $3 \mathrm{~h}$ between the time of release and the time of arrival east of the Dnieper River. During this period the winds reported for the Chernobyl airport were light and variable or caln, and the transport of material in the model was driven almost entirely by the upper-level wind ( $850 \mathrm{mb}$ ) reported for Kiev. There are insufficient wind data to resolve either spatial or temporal variations in wind fields during light winds or to estimate the effect that the upper-level winds may have on transport and diffusion of material released near ground level. Under these conditions, gross errors in the wind fields used by MESORAD to transport the radionuclides cannot be ruled out. 



\section{MESORAD MODEL UNCERTAINTIES}

In general, uncertainties and inaccuracies in models used to estimate doses resulting from releases of radionuclides to the atmosphere are associated with 1) simplified descriptions of atmospheric processes, 2) the random nature of the atmospheric processes involved in dispersion, 3) limited information about the state of the atmosphere during and following the period in which material is released, and 4) limited information on the effluent source. Simplified descriptions of atmospheric processes are required if model computations are to be completed in a timely manner. Sources of numerical uncertainty related to time steps and spacing of receptor nodes used in MESORAD are discussed by Ramsdell, Athey, and Glantz (1983) and Scherpelz et al. (1986). The only source of numerical uncertainty of consequence in the Chernobyl dose assessment is related to the spatial resolution of doses near the reactor. Here the spacing between nodes on the receptor grid is large compared to the size of the plume, and plumes (puffs) may pass between the nodes without being seen by any receptor. Therefore, the model results may not indicate actual dose or dose rate maxima near the reactor.

The uncertainties associated with computation of doses from the concentration distribution in the atmosphere and the surface contamination are small compared with uncertainties in estimating the source term and in the concentration and surface contamination distributions. However, because of the randon nature of the processes that disperse material in the atmosphere, it is unrealistic to expect any model to predict the exact location of or concentration in a plume. Rather, models should be expected to predict average concentrations, and perhaps to provide estimates of the probable variation of concentrations about the average.

The MESOI dispersion model has been tested to distances of about $40 \mathrm{~km}$ from a source using experimental data on atmospheric dispersion collected at the U.S. Department of Energy's Hanford Site in Washington State and the Savannah River Plant in Georgia. Given the detailed meteorological data available at these locations and well-defined source information, MESOI tends to slightly overpredict concentrations in the center of plumes. The median ratio of predicted to observed concentrations for the tests is about 1.4, and 
the distribution of concentration ratios is approximately log-normal (Ramsdell, Glantz, and Andrews 1984). The uncertainty of plume positions (standard deviation of the difference in crosswind location of the predicted and observed plume centerlines) during the same tests increased at a median rate of about $2 \mathrm{~km} / \mathrm{h}$ and had an approximately square-root normal distribution. The distributions of observed-to-predicted centerline concentration ratios and position errors for tests are shown in Figures 9 and 10. The same degree of uncertainty can be assumed to exist in MESORAD dose assessments in near optimum conditions with known release rates.

Model performance can be expected to degrade with reduced information on release characteristics and the state of the atmosphere. To compute doses resulting from the Chernobyl accident, it has been necessary to estimate the specific radionuclides released during the accident, the rates at which various radionuclides were released from the reactor, the duration of the releases, the temporal variation of releases, and the vertical distribution of the releases. Various reports of the accident provide insight into these matters, but they do not provide sufficient detail to permit the source to be considered to be well defined. Errors in any of the assumed source characteristics could change the location and shape of the dose pattern and the magnitudes of the doses.

Errors in the temporal and spatial characteristics of the wind field also lead directly to errors in location and shape of the dose patterns and errors in dose magnitudes. The meteorological data available for dose assessment for the Chernobyl accident are not nearly as comprehensive as the data used in the MESOI tests. Specifically, the meteorological data available for dose assessment for Chernobyl are deficient in that the maximum time resolution is $3 \mathrm{~h}$. The time resolution for the data used in the MESOI model tests was $15 \mathrm{~min}$. The data are also deficient in that they do not support adequate spatial resolution in the wind field. After April 29, data from Chernobyl were no longer available; movement of the effluent from the reactor was estimated using an average of the data from Kiev and Gomel. Under light wind conditions, there is no reason to believe that the winds at Kiev, or at Gomel, or an average of winds at those locations represent the winds at Chernobyl. 


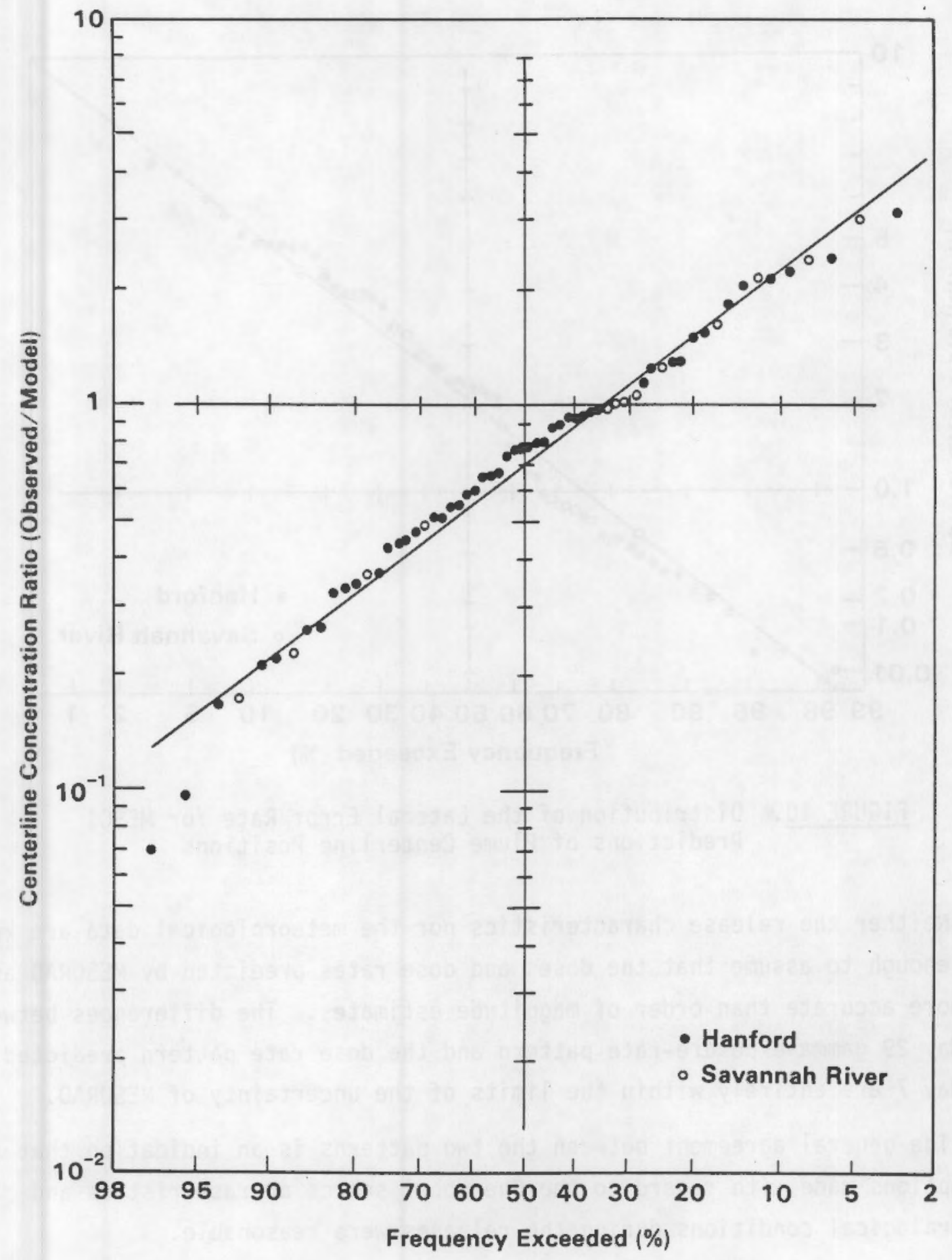

FIGURE 9. Distribution of the Ratio of Observed Plume Centerline Concentrations to Values Predicted with MESOI 


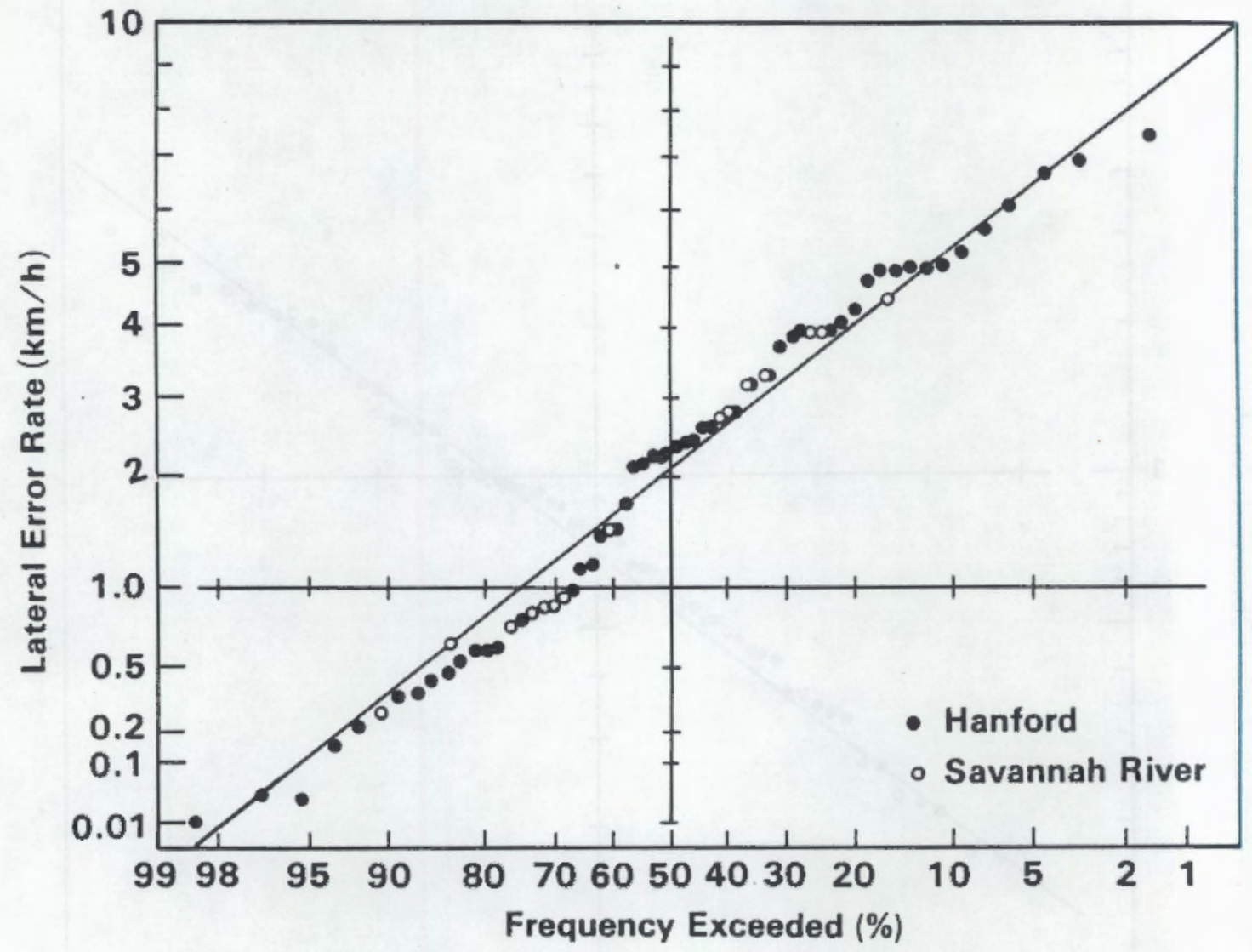

FIGURE 10. Distribution of the Lateral Error Rate for MESOI Predictions of Plume Centerline Positions

Neither the release characteristics nor the meteorological data are known well enough to assume that the doses and dose rates predicted by MESORAD are any more accurate than order of magnitude estimates. The differences between the May 29 gamma-exposure-rate pattern and the dose rate pattern predicted for May 7 are entirely within the limits of the uncertainty of MESORAD.

The general agreement between the two patterns is an indication that the assumptions made with regard to the Chernobyl source characteristics and meteorological conditions during the releases were reasonable. 


\section{REFERENCES}

DOE. 1987. Health and Environmental Consequences of the Chernobyl Nuclear Power Plant Accident. Report to the U.S. Departiment of Energy Office of Heaith and Environmental Research from the Interlaboratory Task Group on Health and Environmental Aspects of the Soviet Nuclear Accident, Washington, D.C.

IAEA. 1986. Summary Report on the Post-Accident Review Meeting on the Chernoby] Accident. International Atomic Energy Agency, International Nuclear Safety Advisory Group (INSAG), Vienna, Austria.

Ramsde11, J. V., G. F. Athey and C. S. Glantz. 1983. MESOI Version 2.0: An Interactive Mesoscale Lagrangian Puff Dispersion Model With Deposition and Decay. NUREG/CR-3344, U.S. Nuclear Regulatory Commission, Washington, D.C.

Ramsile11, J. V., C. S. Glantz and G. L. Andrews, 1984. "Comparison of MESOI Atmospheric Dispersion Estimates With Data and Estimates From Other Models." In Proceedings of the DOE/AMS Air Pollution Model Evaluation Workshop, Kiawah Is land, South Carolina, October 23-26, 1984, DP-1701-1, Savannah River Laboratory, Aiken, South Carolina.

ScherpeIz, R. I., T. J. Bander, G. F. Athey and J. V. Ramsdell. 1986. The MESOPAD Dose Assessment Model. NUREG/CR-4000, U.S. Nuclear Regulatory Cominission, Washington, D.C.

USSR. 1986. The Accident at Chernobyl Nuclear Power Plant and Its Consequences. Information compiled by the USSR State Comittee on the Utilization of At omic Energy for the IAEA Expert's Meeting, August 25-29, 1986, Vienna, Austyia (IAEA Translation). 


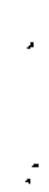


APPENDIX A

METEOROLOGICAL DATA 
APPENOIX A

\section{METEOROLOGICAL DATA}

This appendix summarizes the meteorological input to MESORAD in tabular form. The first column contains the date and time for the meteorological data. The date and time are given as YYMMOOHH where

$Y Y$ is the last 2 digits of the year

MM is the month

$D D$ is the day of the month

$\mathrm{HH}$ is the hour of the day.

A11 times are given as Greenwich Meridian Time (2). Although the Chernobyl accident occurred April 25, 1986, at 23232, for MESORAD simulation purposes it was assumed to occur at 2330z. The data for $0000 z$ on April 26, 1986, were assured to represent the meteorological conditions at the time of the accident. This time is given in the table as 86042600 . The conditions given for each time were assumed to persist for a period of $3 h$, that is, until the next observation.

The second column in the table gives the atmospheric stability class. The atmospheric stability class determines the rate at which diffusion takes place. A low value (e.g., 3) corresponds to relatively rapid diffusion, which is expected during the day, while a high value $(e . g ., 6)$ corresponds to slow diffusion, which is expected at night. The values in the column were assigned on the basis of normal expectations for open country.

The third column in the table gives the thickness of the atmospheric mixing layer to the closest $100 \mathrm{~m}$. The mixing layer thickness undergoes a normal diurnal variation with a minimum at night and a maximum in the afternoon. The values in the table were estimated from afternoon upper-air data for Kiev by assuming a sinusoidal variation between sunrise and sunset and a minimum value of $300 \mathrm{~m}$. A mixing-layer thickness of $800 \mathrm{~m}$ was assumed for the night of the accident to keep the release within the mixing layer.

The fourth column gives the upper-level wind direction and speed as DDDFF where $D D D$ is the wind direction and FF is the wind speed. The direction is 
given to the closest degree, although it is probably only accurate to the closest 10 degrees. The wind speed is given to the closest meter per second. The wind data for reported for the 850-mb level at Kiev (about $1500 \mathrm{~m}$ above ground) were used as the basis for the upper-level wind input to MESORAD after $0900 Z$ on April 26. Before that time the upper-level winds were estimated to be about 090 degrees on the basis of the surface contamination pattern reported as Figure 5.4 in the Soviet report (USSR 1986). The 850-mb wind reported for Kiev at $0000 Z$ on April 26 was 110 degrees. The asterisks indicate interpolation of a wind direction or speed in addition to the interpolation required to estimate winds at the intermediate times.

The data in columns two, three, and four represent conditions that are assumed to be uniform throughout the model domain. Unlike the conditions represented by the data in these columns, the ground-level wind field varies spatially, as well as temporally. The last nine columns in the table contain the available ground-level wind direction and speed (assumed to be measured at $10 \mathrm{~m}$ ) used to derive the surface wind field. These data are given in the same format used for the upper-level wind. Again, an asterisk indicates that a wind direction and/or speed is an estimated value rather than a measured value. 
MESORAD Meteorological Data Input

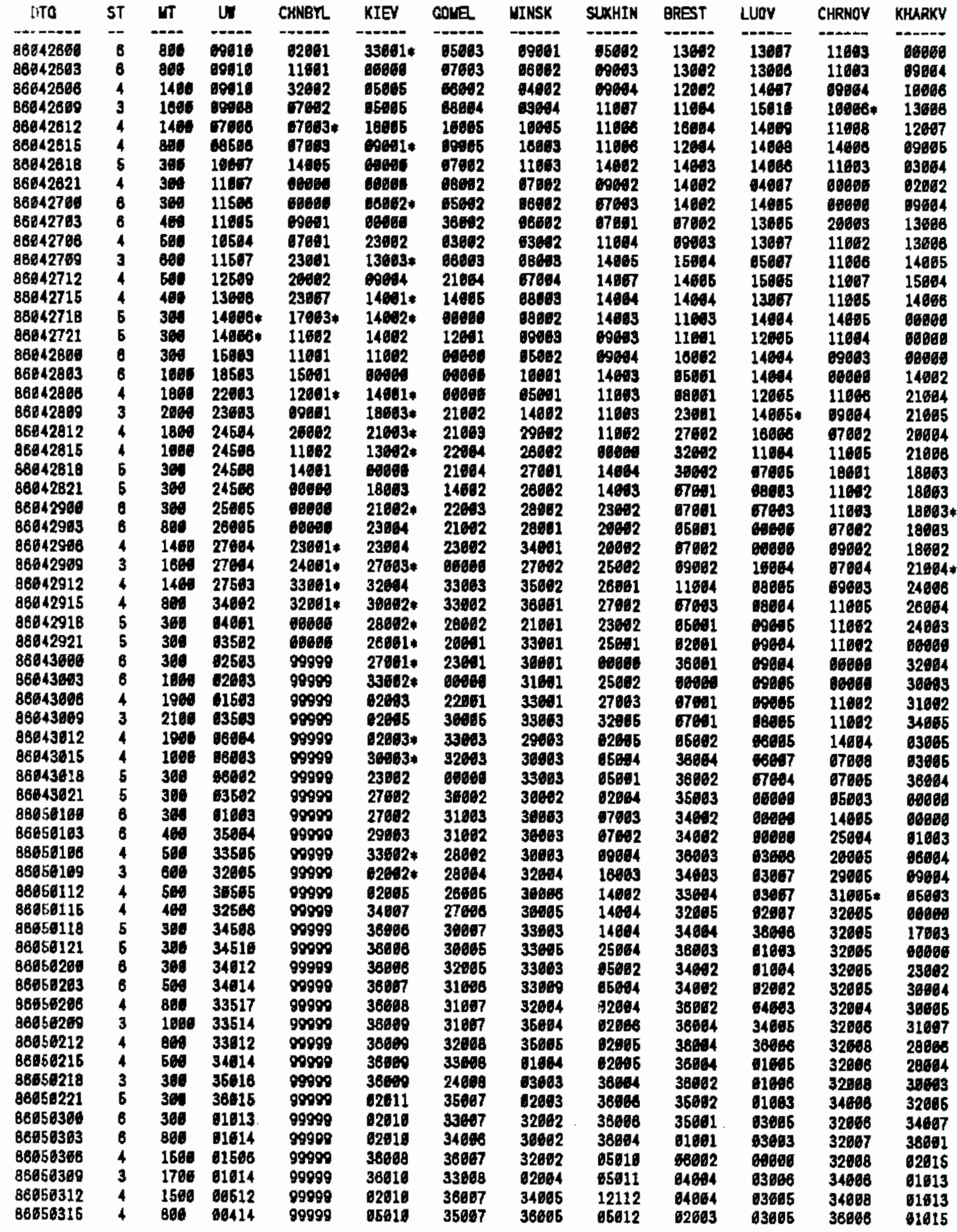


MESORAD Meteorological Data Input (contd)

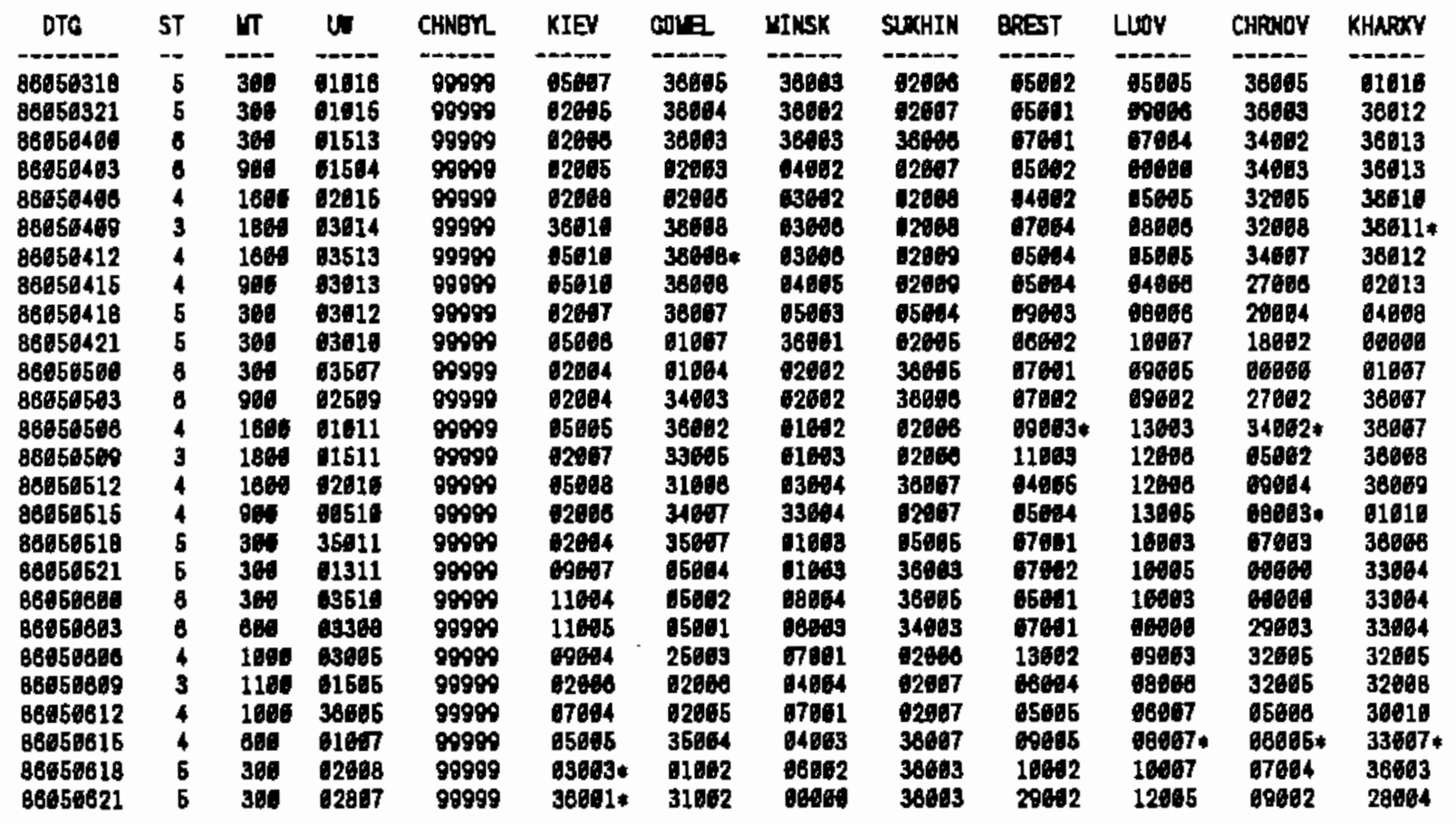


APPENDIX B

MESORAD DOSE ESTIMATES 
APPENDIX B

\section{MESORAD DOSE ESTIMATES}

The following pages contain MESORAD dose estimates based on the atmospheric transport, diffusion, and deposition of effluents released from the Chernoby 1 reactor accident. The dose estimates are cumulative to 00002 on May 7, 1986, and the dose rate estimate is based on material deposited through $0000 z$ on May 7.

MESORAD dose estimates are made on $31 \times 31$ node Cartesian grids. The noda1 estilnates of

1) total whole body dose

2) external dose from the plume (puffs)

3) externat dose from ground shine

4) 50-year whole body dose commitment from inhalation

5) 50-year thyroid dose commitment from inhalation

6) 50-year lung dose commitment from inhalation

7) external dose rate from ground shine

are iresented in this appendix. Presentation of each grid requires four pages. The first page is the northwest quadrant of the grid; the second page is the sout.west quadrant; the third page is the northeast quadrant, and the fourth page is the southeast quadrant. The $x$ and $y$ coordinates are listed for each columin and row of nodes. Coordinate 1,1 is located in the southwest corner of the grid. Within the tables, three numbers are given for each position. The top rumber is the dose or dose rate in units listed at the top of the page. The middle number is the bearing of the node from the center of the grid (node 16,16), and the bottom number is the distance from the center. It shouid be noted that the columns containing data for $x$ coordinate 16 in the northwest and southwest quadrants are repeated in the northeast and southeast quadrants. respectively.

Observations in northern Europe indicated t'lat isotopes of iodine deposited more readily than other isotopes. To replicate this finding, two simulations were performed. The first simulation estimated the doses resulting from the 
iodine releases, and the second estimated the doses from the remaining radionuclides. The dose estimates from the two simulations were combined by a post-processor program to give dose estimates based on all radionuclides. The combined dose estimates are presented here. 


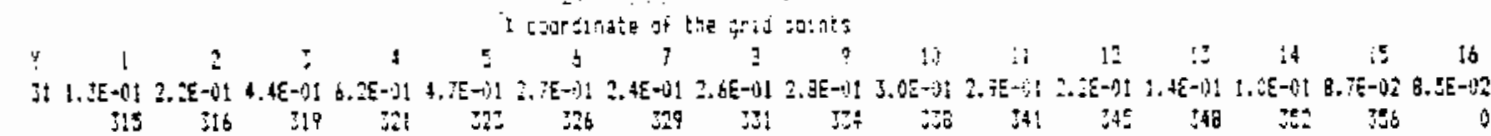

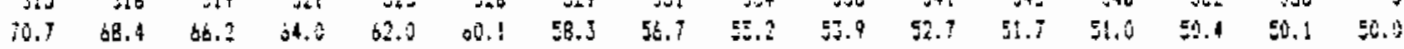

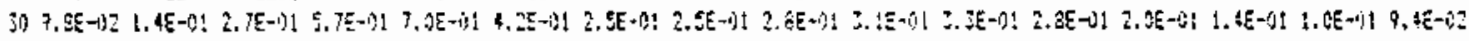

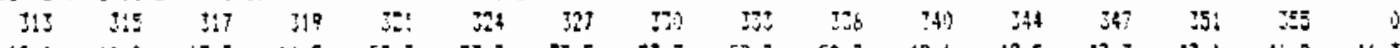

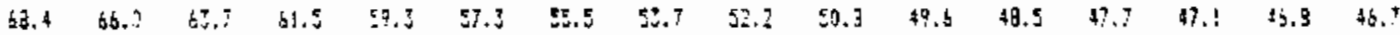

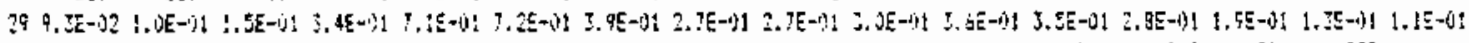

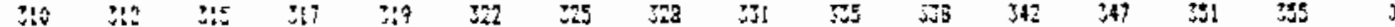

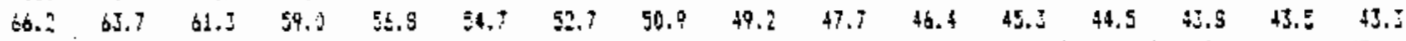

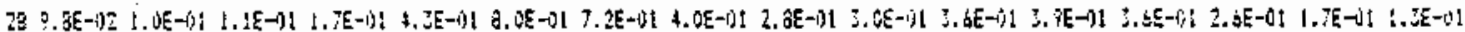

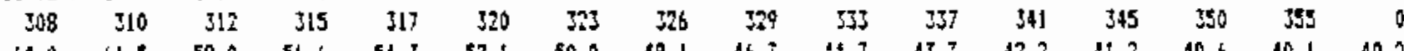

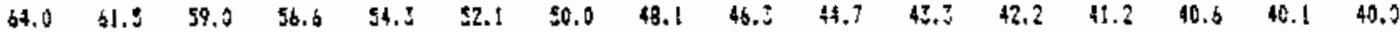

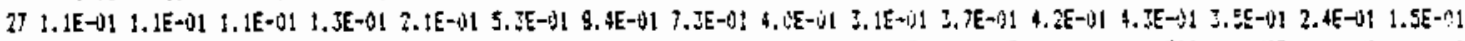

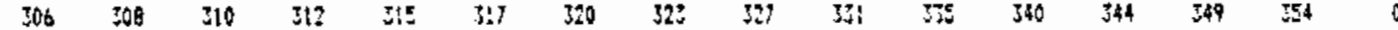

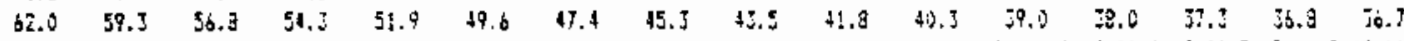

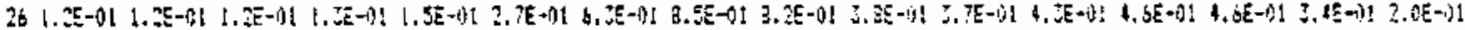

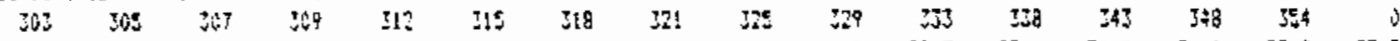

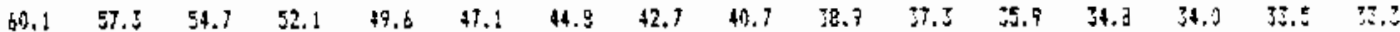

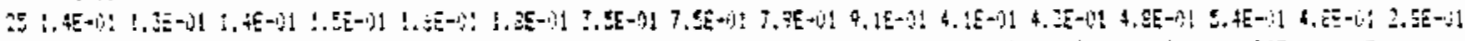

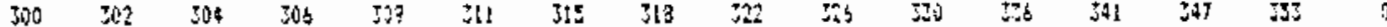

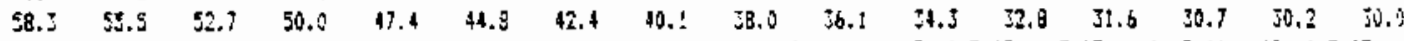

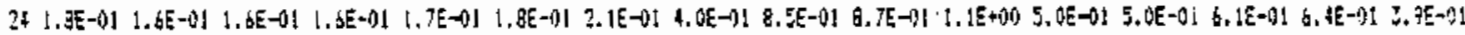

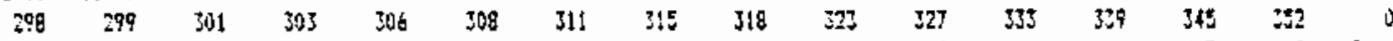

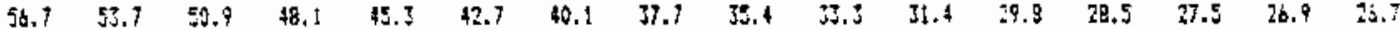

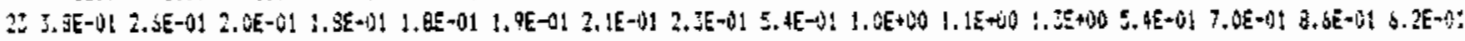

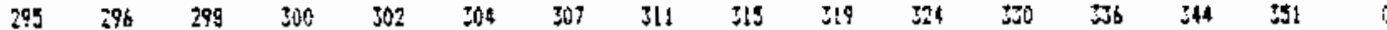

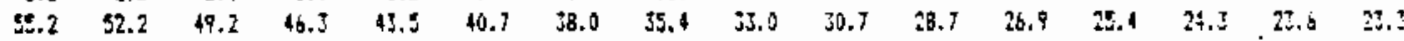

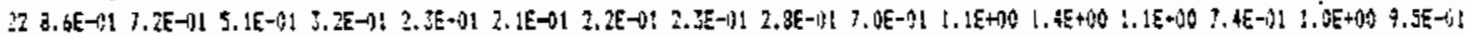

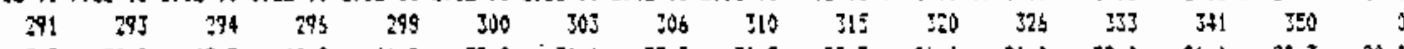

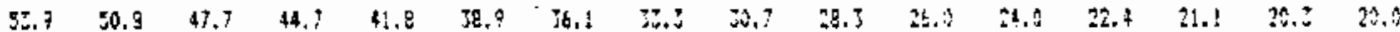

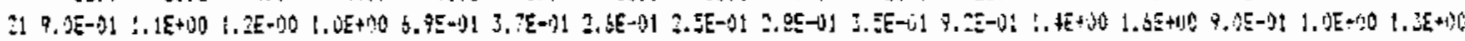

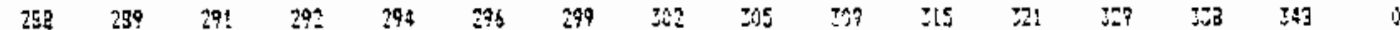

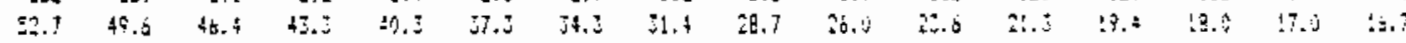

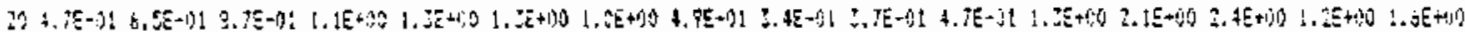

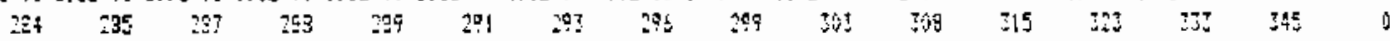

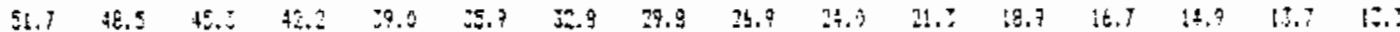

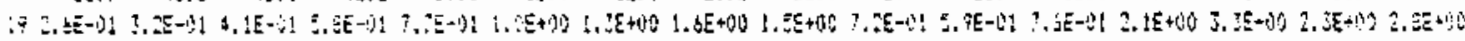

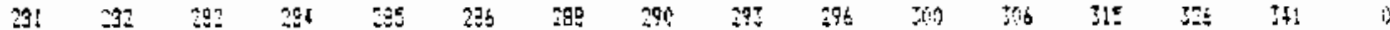

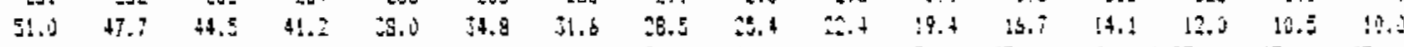

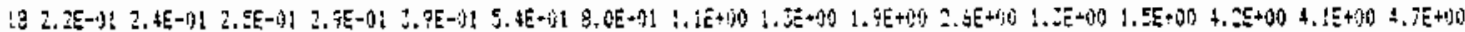

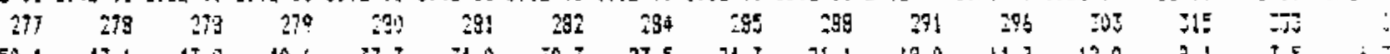

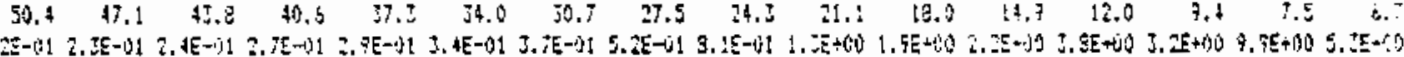

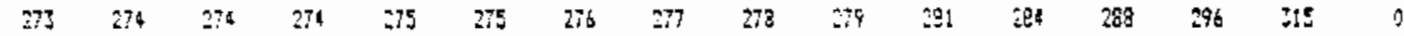

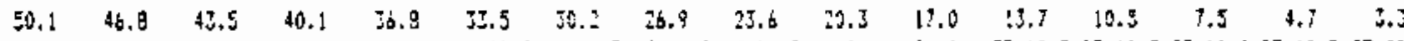

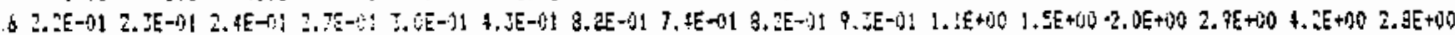

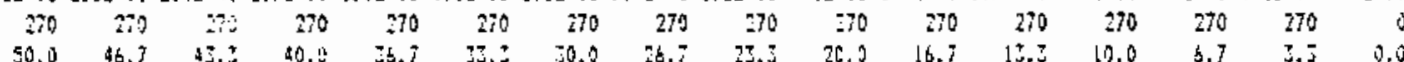

Total whole Body Dose (rem) Estimated for 0000Z May 7, 1986, for the Northwest Quadrant. Bearings are in Degrees and Distances are in Miles from Chernobyl. 


\begin{tabular}{|c|c|c|c|c|c|c|c|c|c|c|c|c|c|c|c|}
\hline \multicolumn{16}{|c|}{ nate of of } \\
\hline$!$ & 2 & j & 4 & $5^{\circ}$ & b & 7 & 9 & 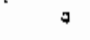 & 16 & $1:$ & 12 & $\therefore$ & $: 4$ & $: \Xi$ & 16 \\
\hline$E-i$ & & & & & & & & & & & & & & & $6.5+\ldots$ \\
\hline 235 & 265 & 245 & 265 & itt & 264 & 265 & $2 \leqslant 2$ & $26:$ & 2so & 259 & 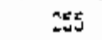 & $\$ 51$ & $\therefore 7$ & 225 & 180 \\
\hline 5.1 & $\$ 6.3$ & 43.5 & $+4,1$ & 75.8 & SE. & 30.2 & $75 . \overline{8}$ & 43.0 & $\because 3$ & 5.3 & 12.7 & 10.5 & 7.5 & 4.7 & 3.2 \\
\hline$\{E-i ;$ & $7 t-9 !$ & $1 . ! E+1 j 0$ & $4.9 E+90$ & $5=0$ & $5.35+152$ & $\therefore ! E+1,3]$ & $1.2 E+100$ & $1.55+00$ & $E E+40$ & $.2 \varepsilon+\infty\}$ & $P E+1,0 ?$ & $\therefore=25+30$ & iztuds & $t, \quad ; E+103$ & $5.2 E+6 D$ \\
\hline $2 \leqslant 2$ & 26! & 261 & $2 \geq 0$ & 399 & TEB & 257 & 255 & $25 t$ & $\tau_{1}$ & 248 & 243 & $\pi 5$ & 225 & 206 & $1 \mathrm{E}_{i}^{2}$ \\
\hline 50.4 & 47.1 & 47.9 & 40.6 & $37 .$. & 34.2 & 30.7 & 27.5 & 24.5 & $21 .:$ & 13.0 & 14.9 & 12.0 & 9.4 & 7.5 & 6.7 \\
\hline $1=4.9 \mathrm{E}-01$ & B. $3 E-1)]$ & $3.3 E+100$ & C. $9 E+10$ & $5.4[+1\} 0$ & $3.95+30$ & $1.1 E+00$ & $9,3 E-111$ & 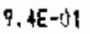 & 1. $4 E+1100$ & $1.2 \varepsilon+10$ & t. $65+130=$ & 2.) $) E+90$ & $2.75+10$ & $3.6 E+190$ & 3.15200 \\
\hline 253 & 257 & 757 & 250 & $2 \pm 1$ & 255 & 251 & 249 & 246 & 243 & 27 & 3 & 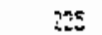 & 217 & $: 92$ & $1 \mathrm{ES}$ \\
\hline 51.2 & 47.7 & 44.5 & 41.2 & 38.2 & 34.8 & 31.5 & 28.5 & 25.4 & -2.4 & 19,4 & 16.: & $14 .:$ & 12.0 & 10.5 & $: 0.2$ \\
\hline$\therefore 4 E+10$ ? & $2.1 E+199$ & $C E+r) 0$ & 2. $\pm \overrightarrow{\vec{c}}+00$ & OE $+0 O$ & $5.7 E-1) 1$ & S. $2 E-0\}$ & $=5.3 k-6 t$ & $6 . \Delta E+M !$ & $7.5 \varepsilon-0 !$ & $9,3 E-d !$ & !. $E+\infty+0$ & 2. CE+ & $3.3 E+100=$ & 2.3E-iid & $\therefore(E+1) D$ \\
\hline 255 & 254 & 252 & 251 & 250 & 248 & 246 & 243 & 240 & 236 & $25 !$ & 235 & 215 & 206 & 194 & 190 \\
\hline 51.7 & 48.5 & 45.7 & 42.2 & $3 r . \hat{v}$ & 35.9 & 32.3 & 29.8 & $3 \$ .9$ & $2 i .0$ & $2: .3$ & 18.7 & IE.: & 14.3 & 12.7 & 13.2 \\
\hline$E-01$ & & $=-v_{i}$ & 3 & $E-4 !$ & ?. . $0 E-U 1$ & 3.9E-U! & 1. $3 E-01$ & 5. $2 E-1) !$ & b.SE-ji! & B. $.5 E-61$ & 1. $2 E+9 y]$ & $2.2 \varepsilon+1 ; 0$ & 2. $.5 E+00$ & B. $3 E+40$ & $2, Z E+100$ \\
\hline 251 & 20 & 243 & 34 & 245 & 245 & 245 & 27 & 234 & 230 & 225 & 213 & 210 & 891 & 191 & 160 \\
\hline 52.7 & 49.6 & 48.1 & 43.3 & 40.5 & 37.2 & $34 . \pm$ & 3.4 & 28.7 & 26.0 & 23.6 & 2i.: & 19.4 & 16.3 & 17.3 & $16 . ?$ \\
\hline$(E-1) 1$ & {$[t-9 t$} & re-1! & $2.9 \pm-31$ & gE-台! & ร.15-ก1 & J. $\{\leqslant-0 !$ & I. $75-121$ & $1.7 \varepsilon=91$ & ร. श्. & i, $\pi 5-91$ & $\therefore, 4 E+\infty A$ & $1.5 E+1.0$ & $1 . T+190:$ & $2.9 E+5 i c$ & (.9E- 9.1$)$ \\
\hline 248 & 296 & 245 & $24 J$ & 241 & 23 & 36 & 233 & 227 & 225 & 259 & $2 ! !$ & 2100 & 198 & $10 \hat{3}$ & ¿j \\
\hline 51.7 & 50.8 & 47.7 & 44.7 & 41.8 & 39.9 & 38.1 & 33.3 & 30.7 & 15.5 & (E.) & 24.0 & 22.4 & $21 .:$ & 3. & 20.3 \\
\hline $9 \therefore . .5 E-01$. & $2 .+[-4] 1$ & $2,4 E-O !$ & 2.5E-3!. & $-2.6 E=-1] 1$ & 2. $8 E-(1) 1$ & $3.2 E-011$ & I. $7 E-31$ & $\$ .4 E-31$ & 5. $4 \mathrm{E}-\mathrm{U1}$ & B. $7 \mathrm{E}-01$ & i. $2 E+100$ & $1.15+50$ & $\therefore . A E+\cdot B$ & $1.5 E+130$ & $1.35 \pm+1\}$ \\
\hline 244 & 243 & $2+1$ & $2: 9$ & 357 & 253 & 332 & 29 & 225 & 219 & :is & 207 & : & :95 & : 69 & :0 \\
\hline 55.2 & 32.2 & 19.2 & 96.3 & 53.5 & 40.7 & i5. 0 & 35.4 & J. & $3 i$ & 28.7 & 26.9 & 25.4 & $3 \$ .3$ & 23.6 & ב.ּ. \\
\hline$i \varepsilon-n \mid$ & 2. $2[\mathrm{E}-\mathrm{l}) \mathrm{I}$ & $z E-0) 1$ & 2. + E- 01 & $5-01$ & 2. $3 E-1) !$ & $\therefore .|E-1|$ & J. $.5 E-0 !$ & 4. $\mid E-B\}$ & 5. $4 \varepsilon-0$ ! & $9.2 E-9 !$ & $3.05-14 ! 9$ & 9..3E-4! & $1.35+00$ & $1,1 E+110$ & 1. $J E+10$ \\
\hline 241 & 240 & 238 & 256 & 233 & 23. & 228 & 375 & 221 & 316 & 212 & 306 & 200 & 394 & 197 & (5) \\
\hline St.7 & 5.7 & 50.9 & 48.1 & 45.5 & $\$ 2.7$ & 40.1 & 37,7 & 35.4 & 32.3 & 31.4 & 29. 8 & 28.5 & 27.5 & 26.9 & 26.7 \\
\hline $2 E-0]$ & -0 & $=-0$ & $\varepsilon-11$ & $E-0 !$ & $E E=01$ & 3. $0 E-01$ & $4 E-01$ & 4. $C E-01$ & $5.3 E-41$ & $9 E-1) 1$ & 6. TE-0! : & 7. & $\lfloor.\{+00$ & 9.jE-i\}! & 1. $0 E+20$ \\
\hline 239 & 277 & 235 & 23 & 230 & 728 & 225 & 221 & $2 ! 7$ & $2:$ & 509 & 203 & 198 & 192 & 186 & $i \theta_{\hat{v}}$ \\
\hline 58.3 & 5.5 & $52 . j$ & 50.0 & $\$ 7.4$ & 44.8 & 42.4 & $40 . !$ & 33.0 & j6.: & 34.5 & 32.8 & $\therefore 1.6$ & 30.7 & 30.2 & ju. \\
\hline وE-M! & 2. OE-0! & 2. $2 \mathrm{E}-0 \mathrm{0}$ & $2 . X E-01$ & $2.5 \varepsilon-0 !$ & $2.7 \mathrm{E}-01$ & Z. SE-91 & $3.3 E-91$ & F. $7 E \rightarrow t$ & $4.6 \mathrm{E}-1) 1$ & $4,95-171$ & $5.7 \varepsilon-01$ & $1.0 E-0:$ & $9,6 E=01$ & 7. $9 E-01$ & 9. 㮫-1!1 \\
\hline 20 & 234 & 272 & 250 & 297 & 225 & $22 !$ & 218 & 214 & 210 & 206 & $30 !$ & 196 & 191 & 295 & :30 \\
\hline 60.1 & 57.2 & 54.7 & 52.1 & 49.6 & $47 .:$ & 44.8 & $4: .7$ & 40.7 & 33.7 & ב. & 3.7 & 34.3 & $3 t .0$ & 活.5 & 33.2 \\
\hline$\{E-\cap:$ & $\therefore . S E-N !$ & $2.2(-1) !$ & [E-1)1 & ...55-4! & 2., & $3.39-1) t$ & $3 . \vec{*}-3)$ & $3.7 E-111$ & 4. $15-0\}$ & 4. $.5-3 i$ & $5.25-1 i$ & b. $\{E-|1 ;|$ & S. 4 tE-1! & b. FE=-1!1 & ; \\
\hline X & 231 & 229 & $2 n ?$ & 275 & 222 & 217 & 216 & $2 ! 2$ & 200 & 204 & $\$ 99$ & 95 & 199 & 195 & 150 \\
\hline 82.8 & 59.2 & 56.8 & 543 & 51.9 & 49.5 & 47.4 & 45.3 & 43.5 & $\$ 1.8$ & 40.3 & 39.2 & $33 .:$ & 3.3 & $\because 3 . j$ & jo.: \\
\hline $9=-31$ & $5-01$ & Es-13! & EE-3\} & $E-1$ & 2. $T_{E}-3 \mathrm{~b}$ & 2. $9 E-01$ & S. $2 E-\hat{0} !$ & 3. $6 E-i !$ & $\partial \varepsilon-11$ & $4 E-i !$ & 4.gE-01 & b. $z[-31$ & IE-JI & 6. $. E-191$ & $7,0 E-4 i$ \\
\hline 231 & 399 & 227 & 235 & 222 & 219 & $2 ! 6$ & 213 & 210 & 200 & $\therefore 2$ & 198 & 194 & : ९9 & 194 & 123 \\
\hline$\$ \$ .0$ & st.s & 59,0 & 55.6 & $54: ;$ & 52.1 & 50.0 & 48.1 & 46,3 & 44.7 & 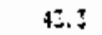 & 92.2 & 41.2 & 40.6 & \$0.: & 40.2 \\
\hline$\varepsilon-01$ & $5-01$ & 2. $1 E-101$ & $\pi E-131$ & $2.5 E-191$ & $2.7(E-1)\}$ & 2.3E-1]! & J. $25-01$ & $3.55=01$ & 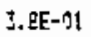 & 4. $\{-0 !$ & 4.55-01 & 5. $2 E+41$ & (. $2 x-11)$ & E. $35-i 1\}$ & 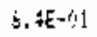 \\
\hline 249 & 227 & 295 & $2: 22$ & 220 & $2 ! 7$ & 244 & $2 ! 1$ & 208 & 204 & $28 t$ & 197 & 192 & :9a & 184 & $i \hat{a} \hat{c}$ \\
\hline 36.2 & 63.7 & $\$ 1 . J$ & 59.2 & 96.9 & 54.7 & 52.7 & 50.9 & 49.2 & 47.7 & 46.4 & 45.3 & 41.5 & 45.3 & \$3. & 43.5 \\
\hline $3 E-0 !$ & CE-OI & !E-1)! & $\bar{x}=01$ & 2. ${ }^{\circ} E-01$ & $2.7 E=01$ & $9 E-191$ & $3.2 \varepsilon-0 \mathrm{I}$ & $45-01$ & EE-11! & $P E-1) 1$ & $4, \ldots-01$ & S. $.6-9: S$ & $s+t E-0 !$ & 5.z $2-i) !$ & 5. (75-1)! \\
\hline 096 & 25 & 222 & 220 & $\therefore 18$ & 215 & $\approx 12$ & 209 & 295 & 203 & 199 & : & 192 & 128 & 194 & 120 \\
\hline 66.4 & b. & $\$ 3.9$ & 61.5 & 59.5 & 57.7 & $\$ 5.5$ & $5 . .7$ & $\mathbf{s . 2}$ & 5. & 49.5 & $\pm 8 . \overline{5}$ & 17.7 & 47.1 & 46.3 & $4 \varepsilon_{1}:$ : \\
\hline 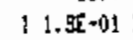 & $O E=-0$ & [E-01 & $E=-j t$ & $4(5-3)$ & 2.7E-0! & 2. $9 E-4) 1$ & 3.1E-11 & 3. tE- & $\therefore . \leq E-i) 1$ & $3 . T E-4 !$ & 4. CE-OU: & $4.95-01$ & $4.7 E=0 !$ & $4.75-01$ & E. $2 E-0\}$ \\
\hline 275 & 223 & 220 & 213 & $2: 6$ & 215 & 210 & 208 & 205 & 201 & :99 & $: 94$ & 199 & $! 97$ & 195 & 180 \\
\hline 70.7 & 58.4 & b6.: & 64.0 & 82.0 & 60.1 & 59.3 & 56.7 & 55.2 & 5.7 & 52.7 & 51.7 & 51.2 & 50.4 & 50.1 & 50.3 \\
\hline
\end{tabular}

Total Whole Body Dose (rem) Estimated for 00002 May 7, 1986, for the Southwest Quadrant. Bearings are in Degrees and Distances are in Miles from Chernobyl. 


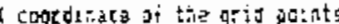

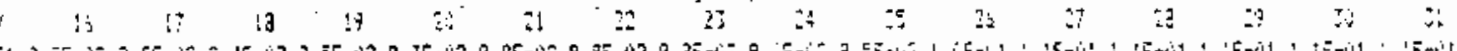

$\therefore$ 3.

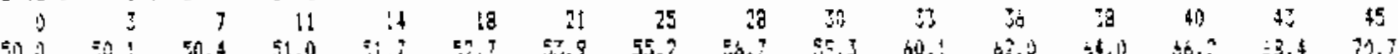

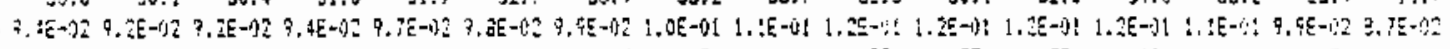

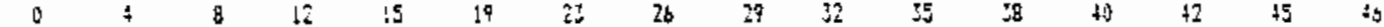

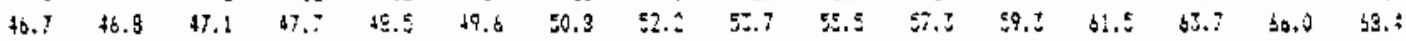

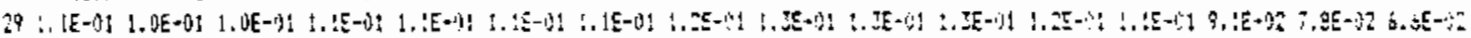

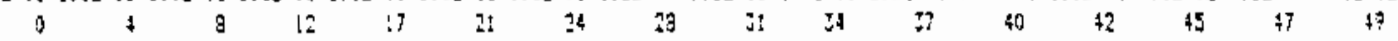

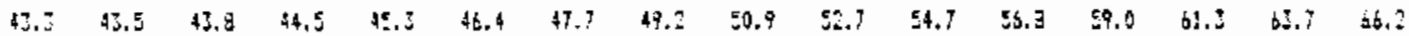

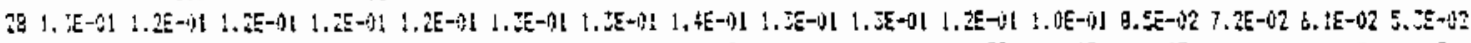

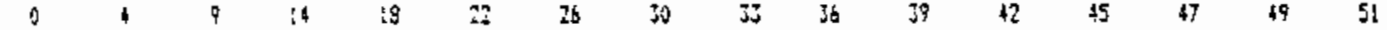

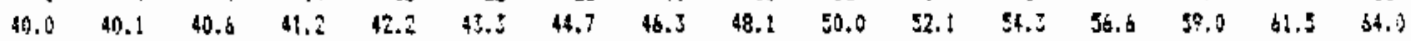

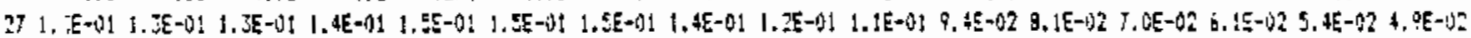

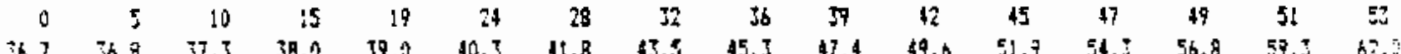

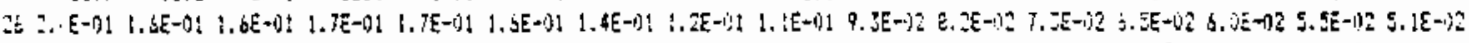

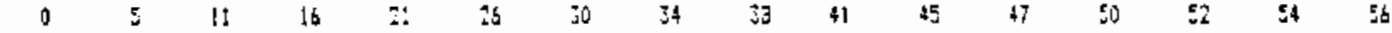

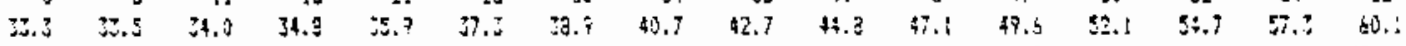

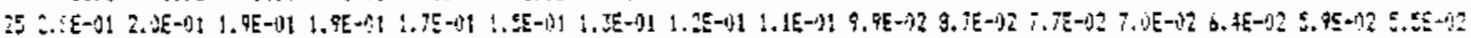

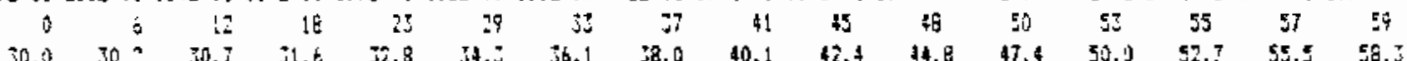

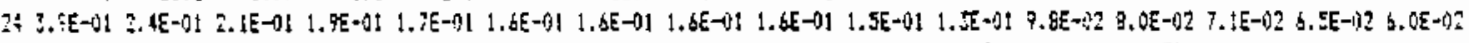

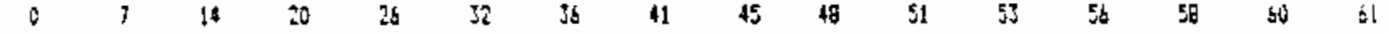

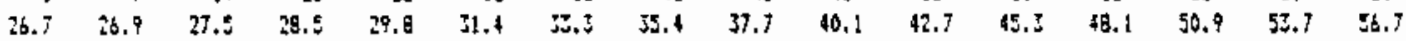

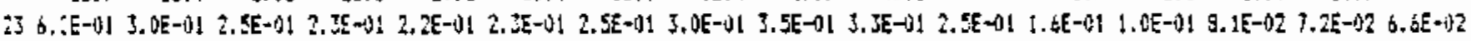

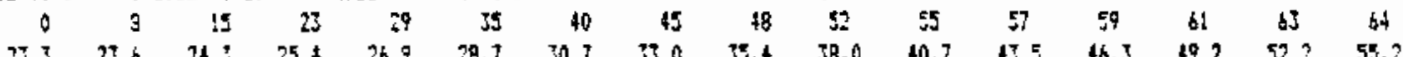

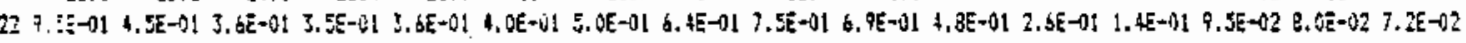

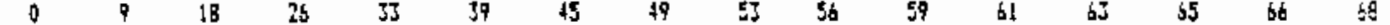

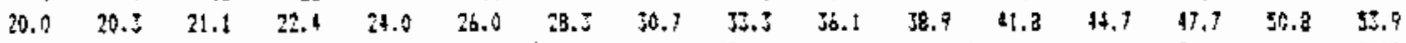
a) :...

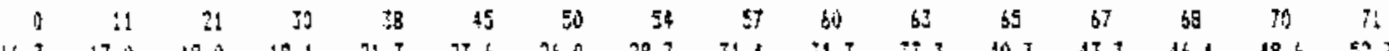

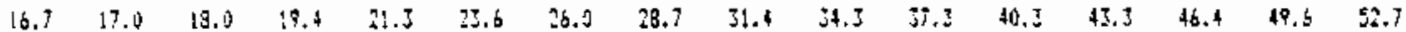

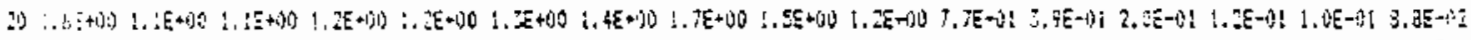

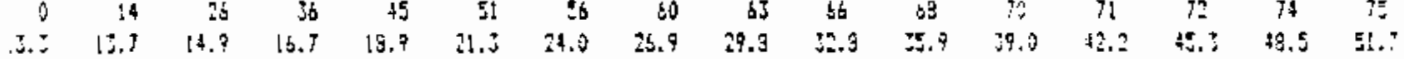

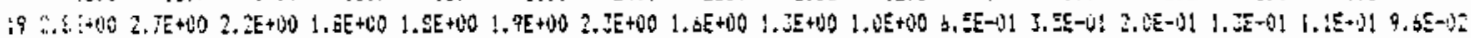

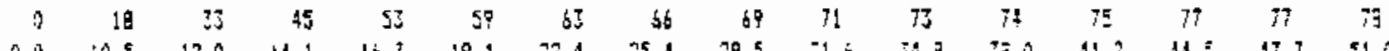

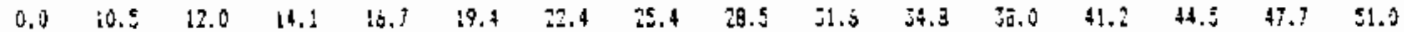

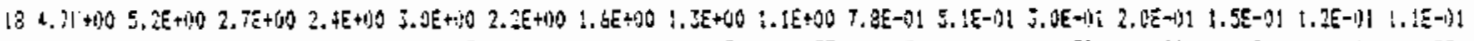

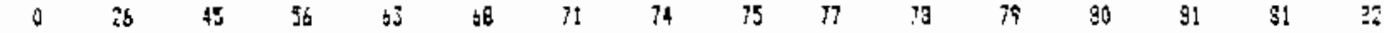

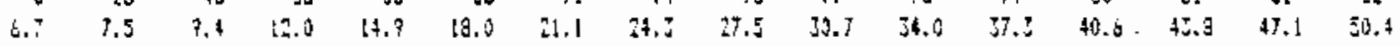

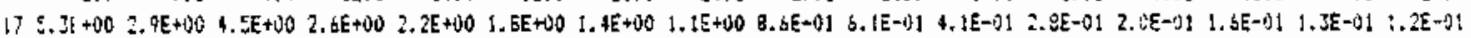

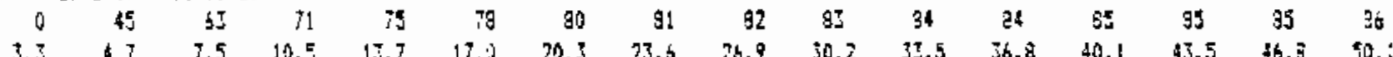

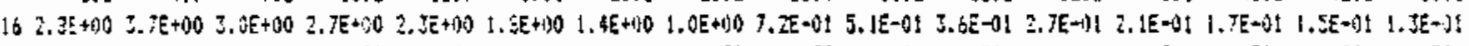

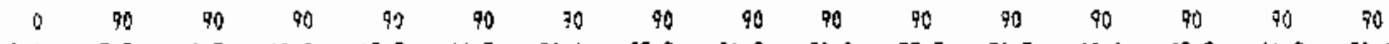

$\begin{array}{llllllllllllllll}0.2 & 3.2 & 6.7 & 10.0 & 13.2 & 16.7 & 20.0 & 23.5 & 26.7 & 30.3 & 37.3 & 36.7 & 40.0 & 43.5 & 46.7 & 50.0\end{array}$

Total Whole Body Dose (rem) Estimated for 00002 May 7, 1986, for the Northeast Quadrant. Bearings are in Degrees and Distances are in Miles from Chernobyl. 


\begin{tabular}{|c|c|c|c|c|c|c|c|c|c|c|c|c|c|c|c|}
\hline & & & & & & 6 & g. & & & & & & & & \\
\hline$! 3$ & 17 & 15 & $1 ?$ & 30 & $2 !$ & 22 & 23 & 24 & 25 & دב & ?7 & 2 & 29 & 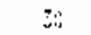 & $3 i$ \\
\hline b. $J E+10 ?$ & ช. $. E+1,0$ & $\therefore=+i 0$ & 2., $E+i g$, & $2.25+100$ & $1.7 E+50$ & {$[2+13]$} & $x=-111$ & b. $\{E-i j t$ & $4, i E+i ! !$ & ¿.5E- I & A.: & $2, \pm-2$ & :. & 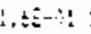 & $\therefore+4 \varepsilon-4:$ \\
\hline 190 & iJs & lio & iNe & 104 & $10 !$ & 79 & 93 & 47 & 76 & 99 & 汇 & 97 & 74 & 74 & 93 \\
\hline 3.3 & 4.7 & 7.5 & 19.5 & 13.7 & 17.8 & 20.2 & 2.6 & 26.7 & jo.? & 3.5 & Z... & 4 4tิ, 1 & 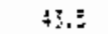 & AS. $\bar{E}$ & E. \\
\hline 6. $2 E+60$ & $4,2 E+10$ & 4. $6 E+1100$ & $3.1 E+50$ & $2.0 E+00$ & $1.2 E+60$ & $1 . \Sigma \varepsilon+?$ & HEt?! & \&. . $\{\varepsilon-n]$ & 4. $\delta \varepsilon-111$ & $\therefore$ Zsćti! & 2, ? ? & 4 & $2.3 E-11 !$ & ! TE-1! & $1.5 \leq-5 !$ \\
\hline 190 & 153 & 135 & $12 \pi$ & $\$ 16$ & 111 & 108 & 105 & 504 & 102 & $10:$ & 100 & 99 & $\because$ & 78 & 7 \\
\hline 8.7 & 1.5 & 9.7 & 12.0 & 14.9 & $: 3.0$ & 21.1 & 24.5 & 21.5 & 30.7 & 34.0 & $\because, 3$ & 40.6 & qंi.a & $47,:$ & $5 x, 4$ \\
\hline$E+10$ & ESt & $7 E+C 0$ & $2.5 E+00$ & 1. $E E+10$ & 1. $A E+\infty O$ & 1. IE+00 & $1.9 \mathrm{E}-\mathrm{ut}$ & b. (EE-1]! & $4,75-4 !$ & $\therefore . B \leqslant-91$ & $\therefore 1 E-\Delta 1$ & $2 . \Delta E-(1) 1$ & $\therefore .25-01 !$ & $1.9 E-1) 1$ & $1,6 \varepsilon-1)$ \\
\hline IaS & 161 & 146 & 10 & 120 & 120 & 116 & 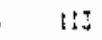 & $\therefore 0$ & : & cos & 165 & 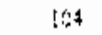 & 108 & $\{1,2$ & 10: \\
\hline ! & 10.5 & 12.0 & 14.1 & 10.7 & 19.4 & 22.4 & 28.4 & 28.5 & 31.0 & 34.3 & 38.0 & 81.2 & 46.5 & 47.7 & E1.2 \\
\hline $12 \quad 2 . T E \div+10$ & $2.55+40$ & $\therefore 4 E+30$ & $1.98+10$ & 1. $6 E+? 0$ & 3. $7 E+1,0$ & $7,65-1) !$ & $7, \Delta E-31$ & 3. DE-9I & $4.8 \varepsilon-t) 1$ & $\Sigma,(\Sigma-1) !$ & $\dot{s}, \dot{\Sigma} \bar{c}-1) !$ & $\therefore 7 E-113$ & $2.2 E-11:$ & C. & 1. $. ? E-1, i$ \\
\hline 280 & 145 & 153 & 143 & 135 & 128 & $\{2\}$ & 119 & 115 & 112 & 111 & 109 & 108 & 107 & ! $f^{2}$ & 149 \\
\hline 13.8 & 13.7 & 16.9 & 16.7 & 18.7 & $2 t .3$ & 24.0 & 96.9 & 29.8 & 32.2 & 35.7 & 39.0 & 42.2 & 45.2 & 43.5 & 5:, \\
\hline$\therefore . Z E+00$ & $2.9 E+00$ & $4 \varepsilon+110$ & $6 E+100$ & $1.4 E+00$ & $\therefore .1 E+00$ & Q. IE-01 & 7. $\bar{E}-41$ & E. TE-UI & 4, $9 E-0 !$ & 4. $(E-1):$ & 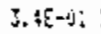 & १โ-1)!? & $4 \bar{E}-\overrightarrow{i j} ; 2$ & :E-1)! & | \\
\hline 190 & 138 & 158 & 149 & I4! & 135 & $n q$ & 125 & 122 & 117 & 114 & 114 & 112 & 111 & $: 07$ & 163 \\
\hline 16.7 & 17.0 & 18.0 & 19.4 & 21.3 & 33.6 & 35.0 & 28.7 & 31.4 & 34.2 & 37.3 & to.2 & $4 ? .3$ & $\$ 6.4$ & 49.6 & 52.? \\
\hline$F E+06$ & $3 E+00$ & JE Hilo & $1.4 E+100$ & $1.2 E+100$ & $O E+t) \theta$ & $t e-0 t$ & $(E-1) !$ & $5.3 E-11$ & 4. $9 \in-1 ?$ & 4. 15-?:? & ...5E-ì1 & AE- & $5 E-012$ & !E+?! & 1.9E-1: \\
\hline 120 & 170 & (b) & $: \varsigma_{3}$ & 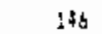 & (5) & 135 & 100 & !is & $12 \pi$ & 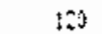 & $\$ 18$ & $1: 3$ & 114 & :I & $\because:$ \\
\hline 20.0 & 30.2 & 24.1 & 22.4 & 24.0 & 26.0 & 28.3 & 30.7 & 3.3 & jis.: & 萢.? & 41.3 & 44.7 & 47,7 & 5.3 & ST: \\
\hline $8+160$ & $2 E+90$ & $i E+00$ & $J E+40$ & $0 E+10$ & OE-01 & $7.7 E-01$ & $6 E=111$ & 5.7E- 01 & 4. 3E-1, & 4. . E-J! & $3.55-20$ & $: E-\Delta 12$ & bE+1!? ? & $i E+\infty 1$ & $Q E-0 i$ \\
\hline 00 & $17 t$ & $: 54$ & 156 & :50 & 144 & 139 & 175 & 13: & 127 & 127 & $! 32$ & 10 & 113 & 116 & !: \\
\hline 22.8 & 22.6 & 24.2 & $2 \pm .4$ & 26.9 & 28.7 & 30.7 & 32.0 & 35.4 & 38.0 & 0.7 & $4 ? .8$ & 46.0 & 49.2 & $5 . .2$ & $55 .:$ \\
\hline a $1.3 E+00$ & $1.4 E+00$ & $1.3 E+0.0$ & $1 .+E+00$ & $3.1 \varepsilon-0 \mid$ & $\Delta . t E=0\}$ & 7. $1 E-0 \mid$ & b. $3 E-0 !$ & 5. 5E-31 & 9. $2(E-3) !$ & $4.1 E-41$ & $3.65=01$ & $\therefore$ :IE-012 & 2. $6 \hat{E}-912$ & 2.JE-4H & $1.98-9 !$ \\
\hline 100 & 172 & 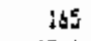 & 159 & 153 & 147 & 143 & 173 & 135 & 131 & $12 B$ & 128 & $12 ?$ & 121 & $1: 7$ & 118 \\
\hline 26.7 & 24.9 & 27.5 & 28.5 & 29.9 & 31.4 & 15.5 & 35.4 & $\$ 7.7$ & 40.1 & 42.7 & 45.5 & 48.1 & 50.7 & 59.7 & 56.7 \\
\hline $1.0 \varepsilon+00$ & 1. $\hat{2} E+00$ & 1. $0 E+00$ & $1.3 E+00$ & B. $\{E-01$ & T, 牙-0i & b. SE-îl & $6.05-1) !$ & $5.5 E-41$ & $4.7 E-01$ & 4. $1 E-01$ & $3.4 E-01$ & S. $1\{-1\} ! 2$ & 2.7E+3! 2 & $2.3 E=11$ & 2.0E-jo\} \\
\hline 180 & 173 & 147 & 161 & 156 & 150 & 146 & 142 & 133 & 13 & 131 & 129 & 126 & 124 & 132 & 150 \\
\hline 30.0 & 30.2 & 30.7 & 31.5 & 32.8 & 34.3 & 36.1 & 39.1 & 10.1 & 12,4 & 44.8 & 47.4 & 50.0 & 52.7 & 55.5 & 59.3 \\
\hline$E E-0\}$ & $6 E-01$ & $O \varepsilon-(i)]$ & $4 E \rightarrow 01$ & 7. SE-01 & 5. $7 E-0$ ? & $2 E-191$ & $6 \in-0 !$ & $5.1 \varepsilon-01$ & 4.SE-A! & $4 ., \partial E-d !$ & EE-91 & Z. $(E-3) ! 2$ & CE-9! 2 & $2.35-151$ & 2. OE- $-1+$ \\
\hline 120 & 174 & 168 & 163 & 158 & 153 & 199 & 145 & 141 & $1 Z B$ & is & $: 2 \pi$ & $1: 9$ & 127 & ! $2 E$ & $\therefore:$ \\
\hline J & 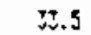 & 34.0 & 34.2 & 25.9 & 37.2 & je.9 & 40.7 & $\{2.7$ & 42.8 & 47.1 & ذ. & 52.1 & 54.7 & 57.2 & bu.: \\
\hline$[(-1)]$ & 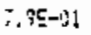 & 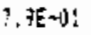 & 3.3F-1)1 & \&. FE-'id & 6. AE-41 & E.6E-i!! & S. $4 \varepsilon-01$ & $4,35-3 t$ & $4,4 E+\{|j|$ & 7.. (E-J) & L.SE-J1 & $2.4 E-i 12$ & 2...E-s:? & 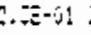 & 2.. $[5-1)$ \\
\hline 290 & $1 \% 4$ & 167 & 154 & 130 & 155 & $15 t$ & 147 & 145 & $1+9$ & 13 & $! \div 8$ & $12 n$ & 20 & $: 23$ & $: \hat{i}$ \\
\hline Es.: & 36.3 & 37.3 & 38.2 & 59.0 & 50.5 & 41.9 & 43.5 & 55.3 & $\$ 7,4$ & 49.5 & $51, ?$ & 54.2 & 50.3 & $\leqslant 9 . j$ & $\{2.2$ \\
\hline$x \in-1 !$ & $3.95-111$ & $3 E-3 !$ & 6. AE-ti & $6 .(E-1) !$ & 5. $3 \mathrm{c}-5 \mathrm{yl}$ & J. & S. $(\varepsilon-1) i$ & $4.75-3 !$ & $4.3-101$ & $3.3[-\hat{1}]\}$ & $\therefore .4 \varepsilon \rightarrow(j 1$ & 3. & Z.SE-J1 2 & 2. $5+4$ & $2.0 E-16$ \\
\hline 500 & 175 & 170 & 165 & 161 & 157 & 195 & 149 & 146 & 143 & 140 & 157 & 125 & $! 52$ & 100 & $: 22$ \\
\hline $4 \hat{2} .0$ & $\$ 0.1$ & 40.6 & 41.2 & $\$ 2.2$ & 43.: & 44.7 & 40.3 & 48.1 & 50.7 & 5.1 & 54,3 & S. É & $8 ? .0$ & 6i. 5 & 44.2 \\
\hline I- & $(E-0) 1$ & $(E-1) 1$ & 5. BE-01 & $5.6 \mathrm{E}-21$ & $5 .+E-01$ & $5.2 E=01$ & 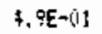 & $4.5[-1)\}$ & 4. iE-QI] & $3.7 \mathrm{E}-01$ & S.ZE-31 & $2.7 E-1) ! 3$ & ¿.SE--): 2 & Q. & $2.3 E-: \mid 1$ \\
\hline 180 & 175 & $17 !$ & tbi & 152 & 158 & 155 & $1 \$ 1$ & 148 & 145 & $1: 2$ & 139 & : & 135 & $1: 2$ & 沙 \\
\hline 43.3 & 43.5 & 43.8 & 44.5 & 45.3 & 46.4 & 47.7 & 49.2 & $50 . \overline{9}$ & 52.7 & 54.7 & 56.3 & 59.0 & 41.3 & $63^{*}$ & bs. 2 \\
\hline$E-0]$ & $5.3 E-0]$ & 6. ZE-0l & $9.55=01$ & $5.35-01$ & 5. IE-01 & 4. $P E=1 !$ & 1.bE-(01 & 4. $5 E-01$ & $\pm .9 E-0 !$ & $3 . \dot{x}=-1)$ & $3.35-31$ & $2.9 E-01 \%$ & 2. EE-U1 2 & $\therefore .25-111$ & $1.75-31$ \\
\hline 180 & 175 & 171 & {$[b]$} & 164 & 160 & $1 \leqslant b$ & 153 & 150 & 147 & 144 & $: 11$ & $1 \$$ & $17 ?$ & $15 z$ & 130 \\
\hline 46.7 & 76.3 & 47.1 & 47.7 & 48.5 & 47.6 & 0.3 & 52.2 & 5.7 & SE.S & $57 .:$ & 59.3 & 61.5 & 6.7 & ) & $8 \bar{E} .4$ \\
\hline CE-1) & $3 E-111$ & 5E-01! & $1 E-0\}$ & $9 E-11 !$ & $8 E-01$ & $E E=0 !$ & $4 E-01$ & : & iE-jt & $4 E-01$ & tE-3] & TE-U! ? & $4 E-0 ! 2$ & $2.15-4 ! 1$ & 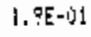 \\
\hline 190 & 176 & 172 & 165 & 165 & ist & $15 a$ & 157 & 151 & 149 & 146 & 143 & 141 & 139 & 196 & 185 \\
\hline 50.0 & 50.1 & 50.4 & 59.0 & 51.7 & 52.7 & 53.7 & 55.2 & 56.7 & 58.3 & 30.1 & 62.0 & 64.0 & $b 6.2$ & \$B.t & $; 0.7$ \\
\hline
\end{tabular}

\section{Total Whole Body Dose (rem) Estimated for $0000 z$ May 7, 1986, for the Southeast Quadrant. Bearings are in Degrees and Distances are in Miles from Chernobyl.}




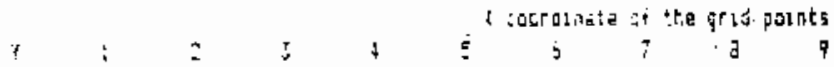

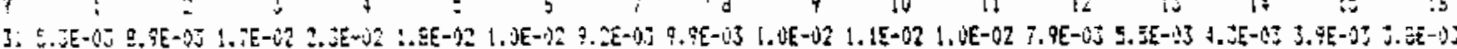

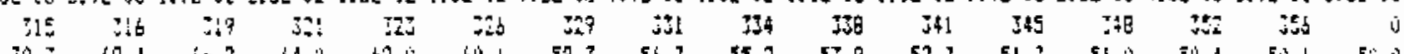

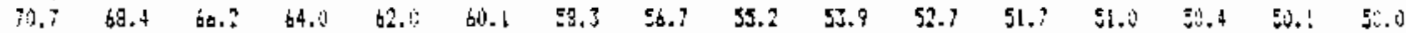

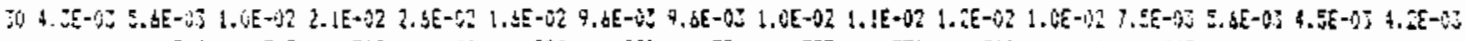

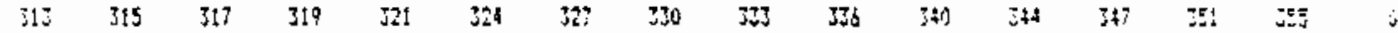

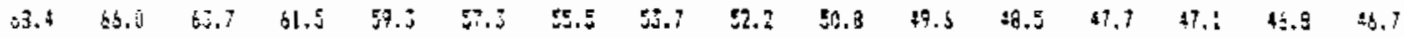

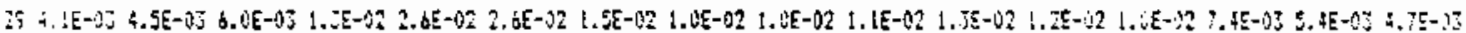

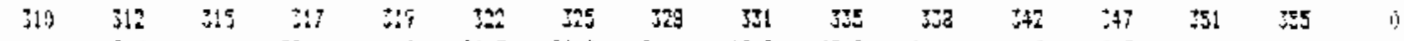

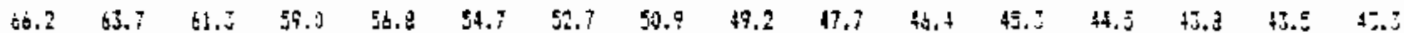

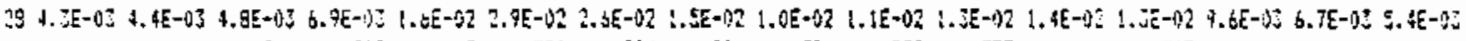

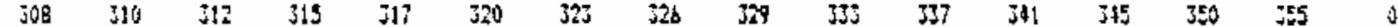

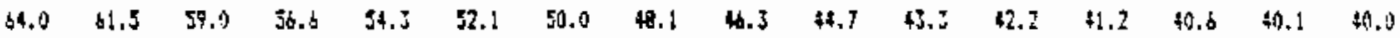

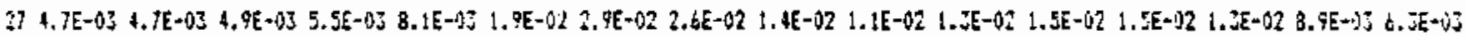

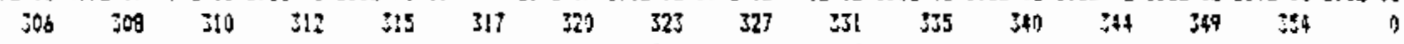

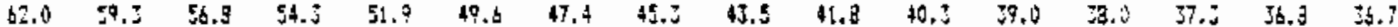

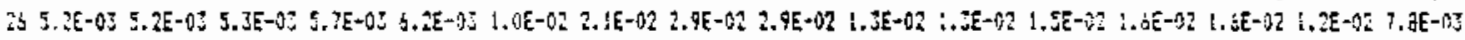

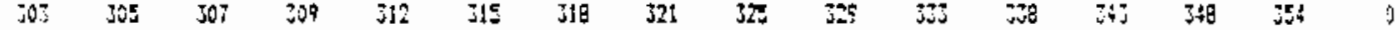

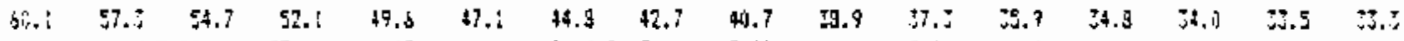

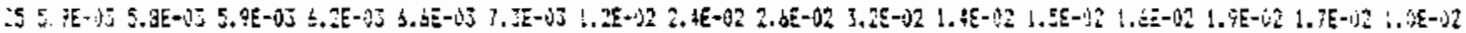

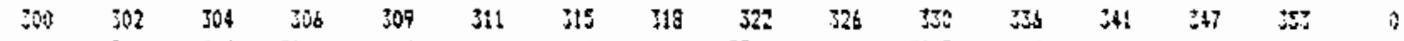

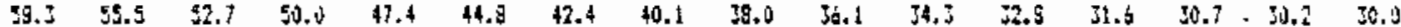

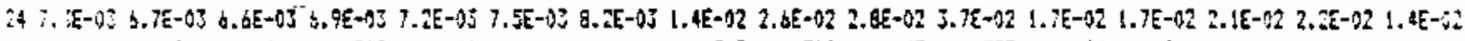

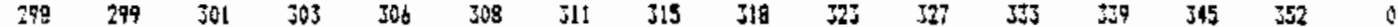

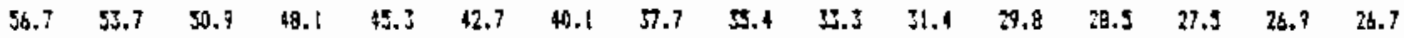

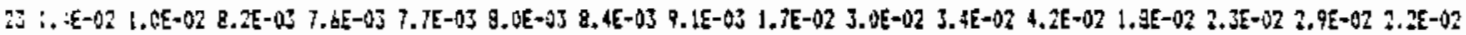
$2952796 \quad 298 \quad 300 \quad 302 \quad 304 \quad 307 \quad 311 \quad 315 \quad 319 \quad 324 \quad 330 \quad 336 \quad 344 \quad 351 \quad 3$

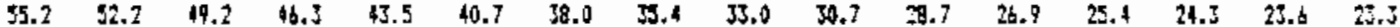

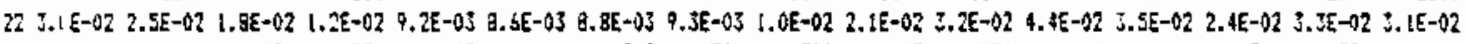

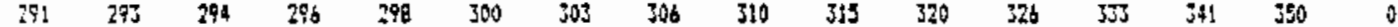

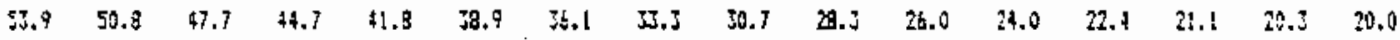

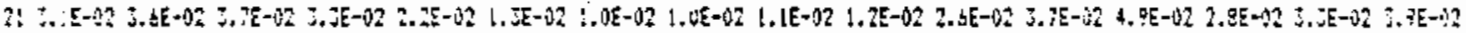

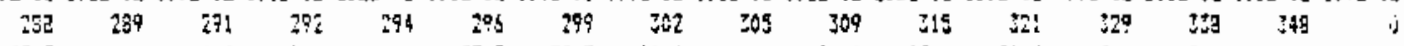

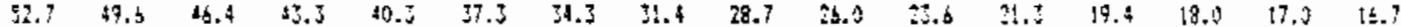

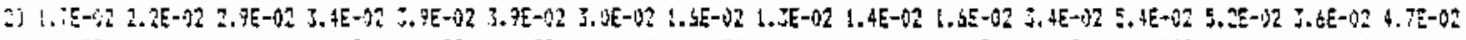

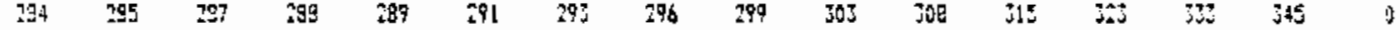

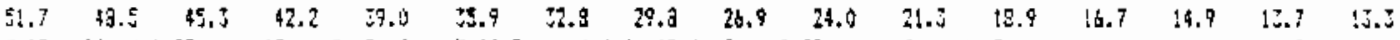

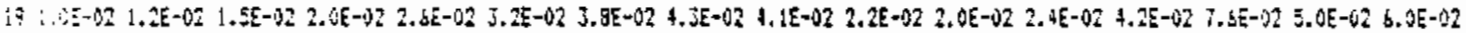

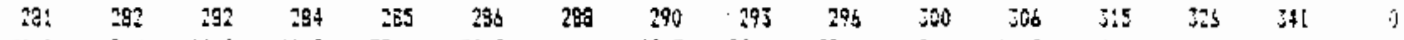

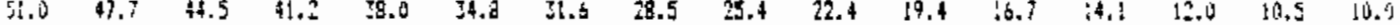

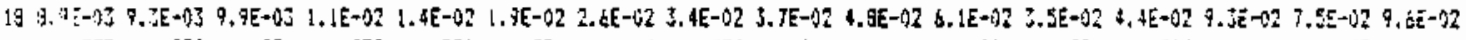

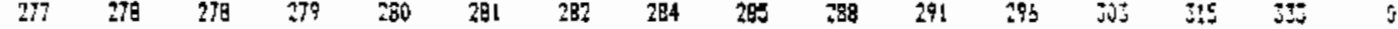

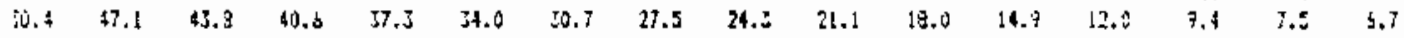

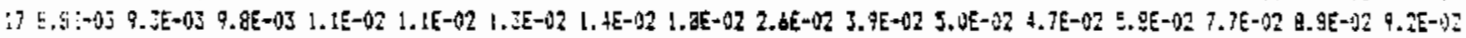

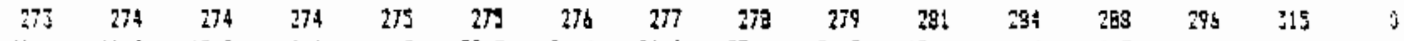
$\begin{array}{lllllllllllllll}\text { id.! } 4.8 & 4.5 & 40.1 & 36.6 & 33.5 & 30.2 & 26.9 & 23.6 & 20.3 & 17.0 & 13.7 & 10.5 & 7.5 & 4.7 & 3.2\end{array}$

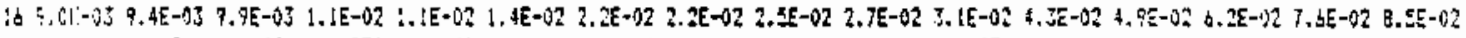

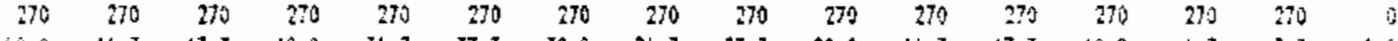

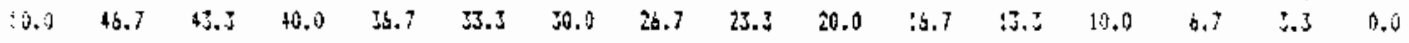

External Dose (rem) from the Plume Estimated for 0000Z May 7, 1986, for the Northwest Quadrant. Bearings are in Degrees and Distances are in Miles from Chernobyl. 


\begin{tabular}{|c|c|c|c|c|c|c|c|c|c|c|c|c|c|c|c|}
\hline \multicolumn{16}{|c|}{ t cogralrate pit the grt1 pourtis } \\
\hline 1 & 2 & j & 4 & \pm & $b$ & ; & 3 & i & $: 0$ & ! & 12 & 15 & $! 4$ & 15 & : \\
\hline & & & 4,4 & {$[-2$} & 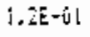 & $\bar{E}-2 \hat{2}$ & $=-j i$ & 5. & $4,1 E-\cdot 2$ & $5.6 E-02$ & $\Xi x-12$ & $1.0 \mathrm{E}-31$ & $3 E-12$ & $4[-172$ & ร. $0\{-1 ! ?$ \\
\hline 266 & 745 & $2 s 5$ & $? \leqslant 5$ & .64 & 264 & ב & 352 & 361 & 240 & 28 & 255 & El & 243 & 220 & $19 \mathrm{~g}$ \\
\hline 50.1 & 96.9 & 43.5 & $4(1) !$ & Ib.9 & 23.5 & 20.2 & 26.9 & 23.5 & 20.3 & 17.0 & 13.2 & i0.5 & 3.3 & 4.7 & 3 \\
\hline $\mathrm{Q} E-\$ 2$ & $6-i j_{i}$ & 3.UE- $) 2$ & $1 .: E-U !$ & [..2E-01 & $\{. j E=f 1$ & ธ. SE- 02 &,$+ \mid E-02$ & $4,9 E-\sqrt{2} 2$ & S.bE -12 ? & b. $15=\sqrt{2} 2$ & 4. $0 \mathrm{E}-02$ & $6.8 E-02$ & $1,3 E-0,2$ & $1,(E-1) !$ & 9.6E- 2 \\
\hline 262 & 261 & $2 s$ & 360 & 259 & 258 & 257 & 255 & 254 & 351 & $2+8$ & 245 & 236 & 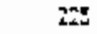 & 326 & : \\
\hline 50.4 & 47.1 & 43.9 & 40.5 & ב. ב.ד & 24.0 & 50.7 & 27.5 & 24.8 & $2 !, 1$ & 19.7 & 14.9 & 12.0 & 9.7 & $? .5$ & $\dot{3 . j}$ \\
\hline $8-\hat{2}$ & & $\{E-72$ & $5-0 !$ & $E-0$ & $2 E=02$ & $5-02$ & $E-t ; 2$ & {$[5-1 ?$} & $4 E-62$ & $5=02$ & ร. $15-42$ & S. $\{E-0\}$ & 1. IE-91 & $E-k$ & 8. $7 E-12$ \\
\hline 350 & 257 & 257 & 255 & 254 & 253 & $2 f !$ & 249 & 296 & 24: & $27 ?$ & 203 & 225 & $21 \%$ & 198 & $: 30$ \\
\hline 51.0 & 47.7 & 94.5 & $\$ 1.2$ & 38.0 & 54.3 & 31.6 & 28.5 & 75. 4 & 22.4 & 99.4 & 15.7 & !4.1 & 2.2. & : A.S & 10. \\
\hline$O E-1) 2$ & $5 . g E-1 \% 2$ & $1,9 \mathrm{e}-5,12$ & $5.7 \mathrm{E}-02$ & $1 E-12$ & 2. $1 E-12.2$ & $2.0[-12$ & $2.0 E-12$ & $\therefore A E-Q Z$ & $2.7 E=42$ & T.JE-4. & 4. $25-12$ & 6. $7 \varepsilon-\tilde{u} 2$ & $1.1 E=01$ & B.SE-112 & 9. $\pi t-j 2$ \\
\hline 25 & 254 & 252 & $25 !$ & 250 & 248 & 246 & $24 j$ & 240 & $2 \pi$ & $7 * 1$ & 225 & 216 & $20 b$ & 194 & !91) \\
\hline 51.7 & 48.5 & 45.: & 42.2 & 39.0 & 55.9 & \pm 2.8 & 29.9 & $2 b .9$ & $24.1)$ & 21.3 & 19.7 & 16.7 & 14.9 & 13.7 & ب.? \\
\hline $5 E-02$ & $1 E-02$ & $75-92$ & $5 E-82$ & $5 E-102$ & $E E=02$ & $5-02$ & $7 \varepsilon-02$ & $2.0 E-02$ & $+E-32$ & $9 E-02$ & $\therefore .9 E-02$ & $7.55-42$ & $6 E+112$ & 8. $0 \mathrm{E}-8 \mathrm{n}$ & $7.25-125$ \\
\hline $25 !$ & 250 & 243 & 247 & 245 & 243 & 240 & 237 & 34 & 30 & 235 & $2: 3$ & 2100 & $20 !$ & $19 !$ & 190 \\
\hline 52.7 & 49.6 & 44.4 & 43.3 & 10.3 & 37.3 & 34.3 & 31.4 & 29.7 & 26.0 & 23.6 & 21.3 & 19.9 & 19.0 & 17.0 & 16.7 \\
\hline$[-0,2$ & {$[\cdot ?]$} & $t-4$ & $(2-1)^{2}$ & $=-4 \pi$ & $=-7 / 2$ & $=-1+2$ & $E-12$ & $3 E-02$ & $2 E-32$ & $T E-02$ & $:=-12$ & $\{-02$ & $x-n 2$ & $5 E-122$ & b. $\{E->\}$ \\
\hline 246 & 246 & 245 & 243 & 241 & 279 & $2 \pi 6$ & 253 & 229 & 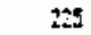 & 219 & 213 & 206 & 199 & $! 99$ & 50 \\
\hline 52.7 & E\{.a & 47.7 & 44.7 & $\$ 1.9$ & 0.7 & 法.1 & 3.3 & 30.7 & 28.3 & 26.0 & 24.8 & 22.4 & 21.1 & 20.3 & $20, j$ \\
\hline$E-02$ & $E=10$ ? & $E=02$ & $15-02$ & $2 E-02$ & $1.2 \mathrm{E}-02$ & 1.jE-02 & $1.55-02$ & $7 E-02$ & $15-02$ & ذ.:25-02 & 4.5E-1.2 & $3 . \pi E-12$ & $4.75-12$ & $5 . ! E-x^{2}$ & 5. $I \Sigma+i 2$ \\
\hline $2 \$ 4$ & 243 & 241 & 119 & 257 & 255 & 232 & 299 & 25 & 220 & 215 & 209 & 203 & 195 & Iag & 120 \\
\hline 55.2 & 52.2 & 49.2 & $46 x j$ & +3.5 & 40.7 & 50.0 & 35.4 & 33.0 & 30.7 & 20.7 & 26.9 & 23.4 & $24 . ?$ & 23.0 & 23.3 \\
\hline$E E-03$ & $9,3 E-93$ & $E-52$ & $1 \varepsilon=02$ & $1 E-1) 2$ & $1.25-02$ & $1,2 \varepsilon-02$ & $G E-12$ & $7 \varepsilon-02$ & $1 E-92$ & $3.5 E-122$ & $3.0 E=02$ & $3.3 \varepsilon-02$ & $3 E-12$ & $\int E-\sqrt{2}$ & +E-02 \\
\hline 341 & 240 & $2 \div 8$ & $23 b$ & 235 & $23 !$ & 229 & 225 & 221 & 216 & 212 & 206 & 300 & 194 & 197 & 150 \\
\hline 56.7 & 3.7 & 50.7 & 80.1 & 45.3 & 42.7 & 40.1 & 37.7 & 75.4 & 35.3 & 32.4 & 79.3 & 29.5 & 27.5 & 26.9 & 26.7 \\
\hline $05-03$ & $6-03$ & $E-03$ & $1 \varepsilon-02$ & $t E=102$ & $1 . \pi-02$ & $3 E-m$ & $4 E-02$ & $b \varepsilon=92$ & ZE-92 & $3 E-022$ & $4 e-02$ & 9E-152 & $95-62$ & $.3 E-02$ & $3.7 E-62$ \\
\hline 229 & 27 & 235 & 272 & 250 & 228 & 235 & $22 !$ & 17 & 21: & 209 & 203 & 198 & 192 & 26 & 190 \\
\hline 38.3 & $5 s .5$ & 52.7 & 90.0 & 47.4 & 44.8 & 42.4 & 40.1 & 38.0 & 36.1 & 34.2 & 32.8 & 31.5 & 30.7 & 30.2 & 30.0 \\
\hline$E-1) 3$ & {$[-1)$} & $E-0 z$ & $E+02$ & $\mid E-0)$ & $1.3 E-02$ & $\hat{\varepsilon}-02$ & t. $4 \varepsilon-02$ & $\Delta E-\imath 2$ & $9 \varepsilon-02$ & 2. $\Delta E-022$ & $2.2 E-02$ & $7 E-02$ & $E-02$ & $q E=\Delta ?$ & 3. $2 E-6+2$ \\
\hline 23b & 224 & $2: 2$ & 230 & ะ27 & 225 & $22 !$ & 218 & 214 & 2110 & 200 & 201 & 196 & [91 & 165 & 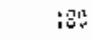 \\
\hline 30.1 & 57.3 & 54.7 & $52 . !$ & 49.6 & 47.1 & $\$ 4.9$ & 42.7 & 4i). & $33 . ?$ & 37.2 & $x$ & 34.3 & 34,0 & D. & ני. \\
\hline $2 E-03$ & $3 E-03$ & $9 E-03$ & 1. : $: 5-02$ & 1.: $: E-02$ & $1.2 E-02$ & {$[E-1] 2$} & $1,4[-1) 2$ & $6 E-142$ & $7 \varepsilon-1 \hat{2}$ & $9 E-92=$ & $\therefore 1 E-02$ & $E E-62$ & $: \varepsilon-22$ & SE-32 & $75-8]$ \\
\hline 233 & $25 !$ & 229 & 227 & 225 & 222 & 219 & 216 & 212 & 208 & 204 & 199 & 195 & $: 90$ & $: 95$ & $13 j$ \\
\hline 62.0 & 59.3 & 50.9 & 54.3 & 51.9 & $\$ 9.5$ & 17.4 & 45.3 & 43.5 & 11.8 & 40.2 & 39.0 & 39.4 & 3.5 & I. 2 & is.? \\
\hline$d E-0 \vec{j}$ & $3 t-v 3$ & $9 E-9 j$ & $15-12$ & $15-112$ & 1. $2 \mathrm{E}-02$ & $3 E-102$ & $4 E-12$ & $5 E-1,2$ & $j E-02$ & $1.38-02 ?$ & ר. & 2. $1 \mathrm{~A}-0$ ? & $2.7 E-02$ & $1 .: E+12$ & 2.SE-VI \\
\hline 231 & 239 & 227 & 22 & 222 & 219 & $21 \dot{ }$ & 213 & 250 & $20 s$ & 202 & 199 & 194 & 159 & 104 & [8] \\
\hline 64.0 & 61.5 & 59.0 & 56.6 & 54.2 & $5 . .1$ & 50.0 & 49.1 & 46.2 & 44.7 & 43.5 & 12..2 & 41.2 & 40.5 & $4 i, 1$ & 44.3 \\
\hline$E-0$ & $E-1$ & $95-05$ & $1 E-102$ & $1.1 E-02$ & 1.2E-02 & 1.3E-02 & $1.4 E=02$ & $1.5 E-132$ & ¿.6E- 02 & $1.7 \mathrm{E}-12$ & 1.3E- $\sqrt{2}$ & $2 . J E+02$ & $2.4 E-02$ & S.DE- -2 & $2.45-92$ \\
\hline .29 & 227 & 205 & 322 & 921 & 217 & 214 & 211 & 200 & 204 & 201 & 197 & 192 & 189 & 194 & (3) \\
\hline 66.2 & 63.7 & $b 1.3$ & 59.0 & 56.8 & 54.7 & 52.7 & 50.9 & 49.2 & 47.7 & 96.4 & 45.3 & 44.5 & 43.8 & 43.5 & $\$ 3 . j$ \\
\hline $7 E-03$ & $2 E-03$ & $g E-0]$ & $O E-02$ & $1 E-02$ & 25-0? & $3 E-1)$ & $4 E-1)$ & $5 E-102$ & SE-102 & $7 E=02$ & $7 \varepsilon-02$ & $\pi E-2 ?$ & $1 E-32$ & $: \Sigma+1) 2$ & 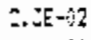 \\
\hline $2 a b$ & 225 & 222 & 200 & 213 & 215 & 212 & 209 & 296 & 203 & 179 & 145 & 192 & 198 & let & : \\
\hline 58.4 & 66,0 & 63.7 & 61.5 & 59.5 & 57.3 & $5 * .5$ & 8.7 & $\leq 2.2$ & 50.9 & 49.6 & 46.5 & 17.7 & 47.1 & 16.3 & $\$ 6.7$ \\
\hline & E- & $=-20$ & $U E=0$ & $1 E=02$ & $2 E-02$ & $3 E-022$ & $4 E-92$ & 1. $5 \varepsilon-02$ & $1.55-\diamond 2$ & I. $E E-n 2$ & 1.jE-g? & $2.0 E=02$ & $1.3 E-02$ & 1. $9 E-\sqrt{2}$ & $2.15-132$ \\
\hline 22 & 22 & 22 & $\eta !$ & 216 & 243 & 210 & 209 & 205 & 201 & 199 & 154 & 191 & 187 & 193 & 180 \\
\hline 70.7 & 68.4 & 6́. 2 & 84.0 & 52.0 & 60.1 & 58.3 & $5 b .7$ & 55.2 & 53.9 & 52.7 & 56.7 & 51.0 & 50.4 & 80.1 & 50.8 \\
\hline
\end{tabular}

External Dose (rem) from the Plume Estimated for 00002 May 7, 1986, for the Southwest Quadrant. Bearings are in Degrees and Distances are in Miles from Chernobyl. 


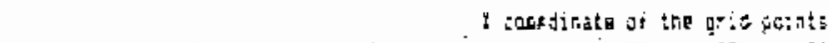

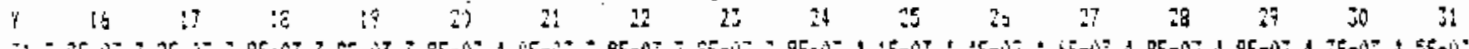

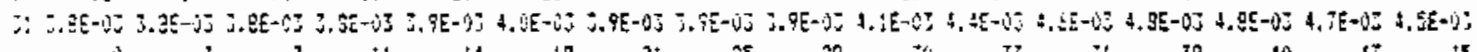

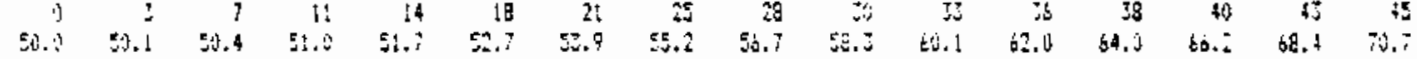

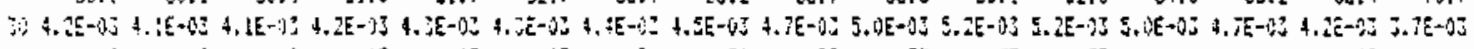

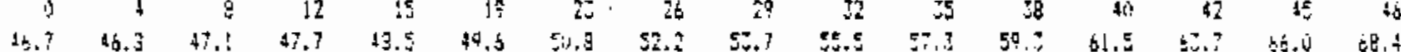

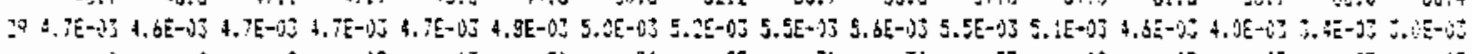

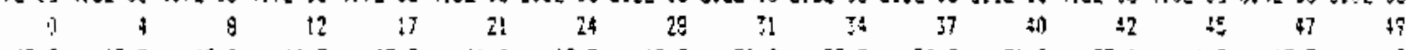

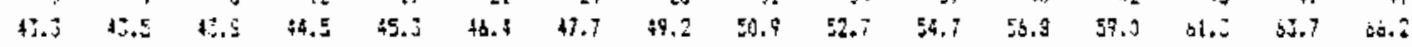

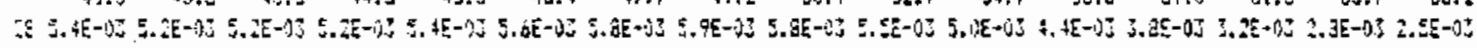

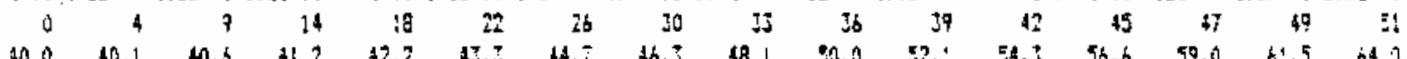

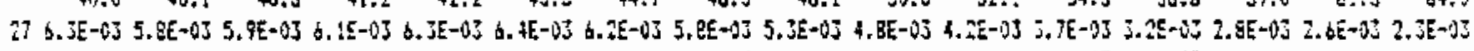

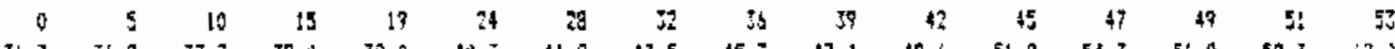

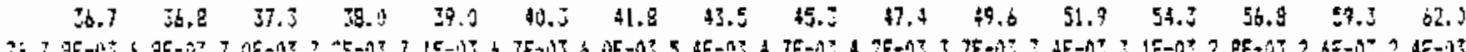

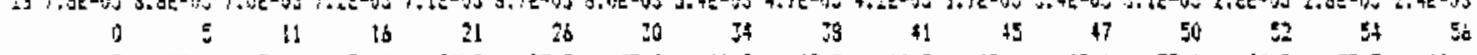

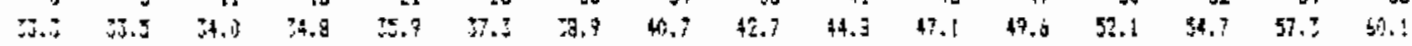

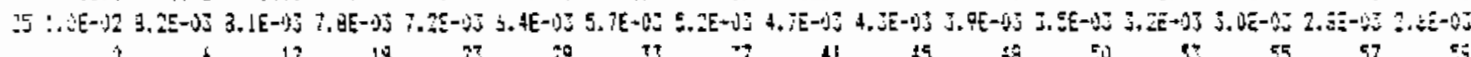

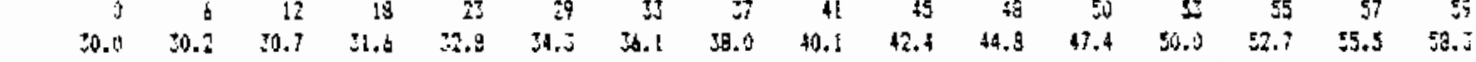

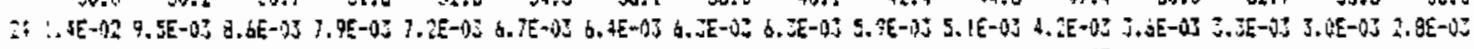

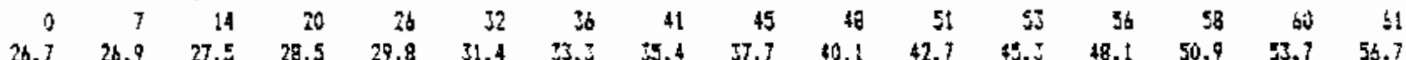

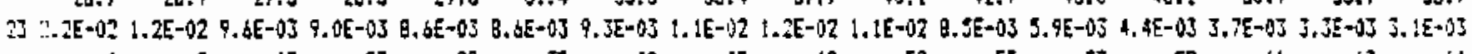

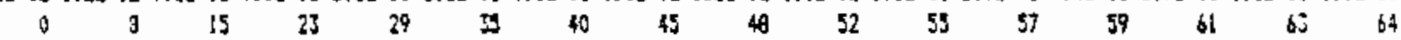

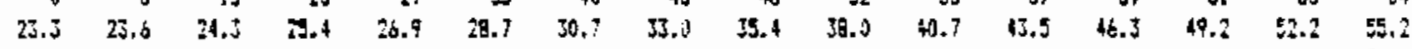

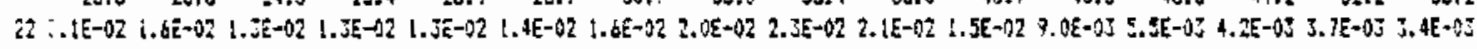

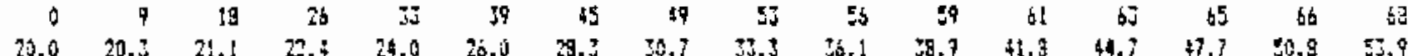

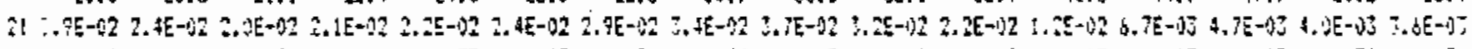

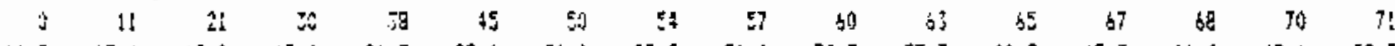

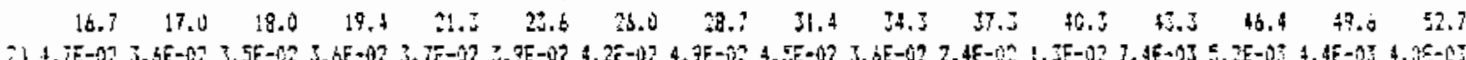

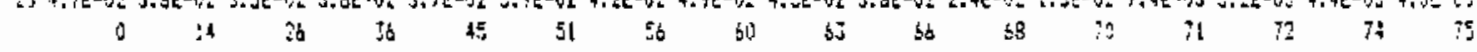

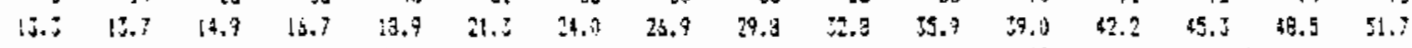

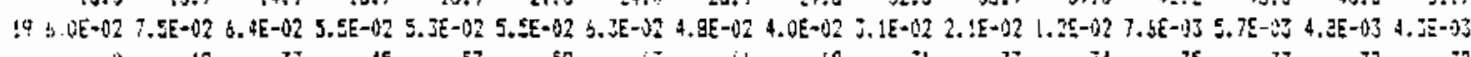

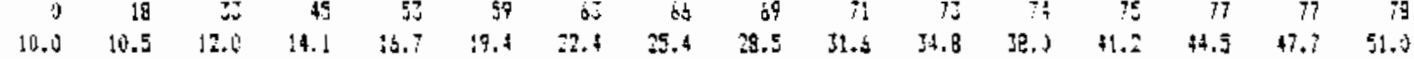

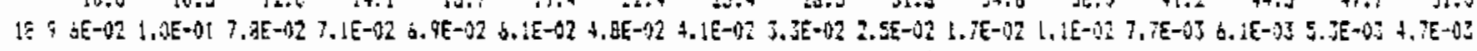

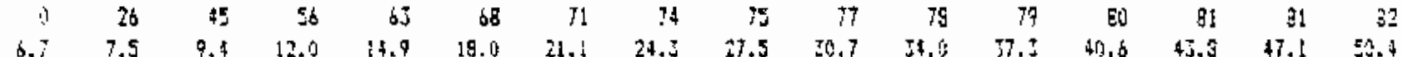

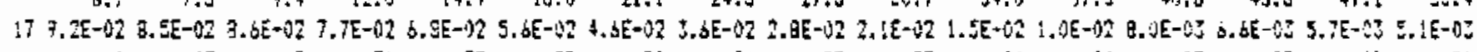

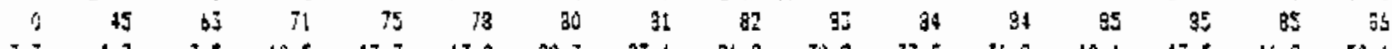

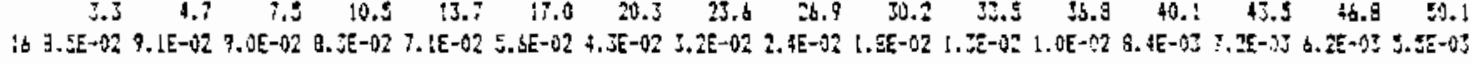

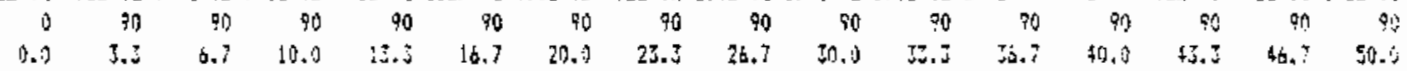

External Dose (rem) from the Plume Estimated for $0000 Z$ May 7, 1986, for the Northeast Quadrant. Bearings are in Degrees and Distances are in Miles from Chernobyl. 


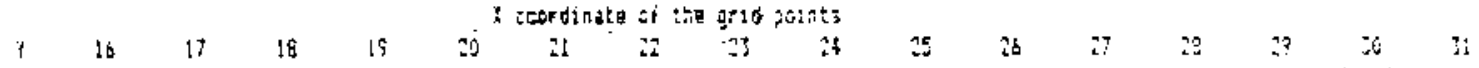

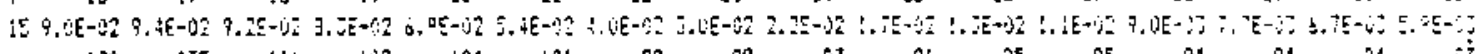

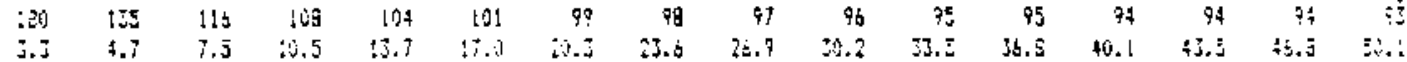

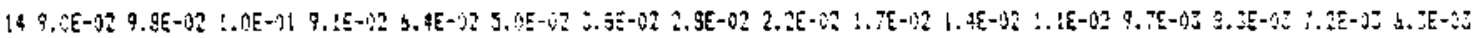

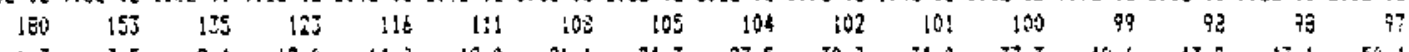

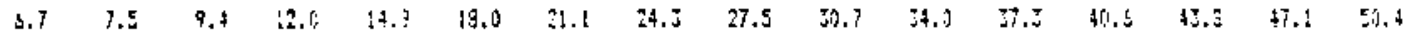

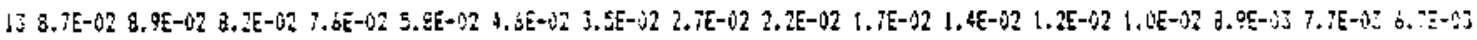

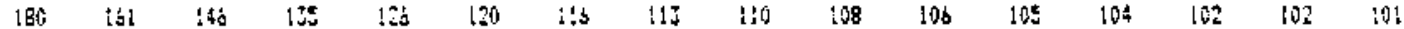

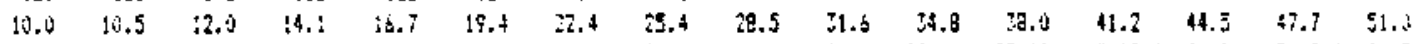

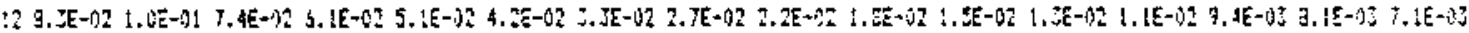
$\begin{array}{llllllllllllllll}180 & 165 & 153 & 143 & 155 & 126 & 125 & 119 & 116 & 113 & 111 & 109 & 108 & 107 & 105 & 104\end{array}$

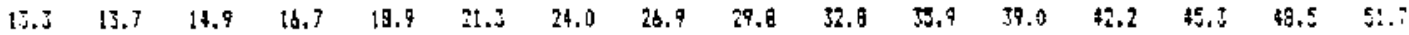

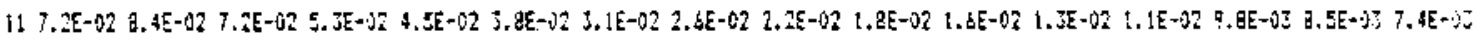
$190 \quad 168 \quad 158 \quad 149 \quad 241 \quad 135 \quad 127 \quad 125 \quad 122 \quad 119 \quad 116 \quad 114 \quad 112 \quad 112 \quad 109 \quad 163$

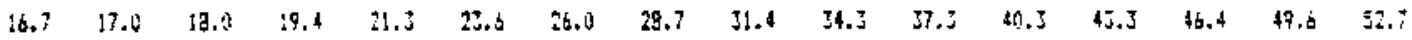

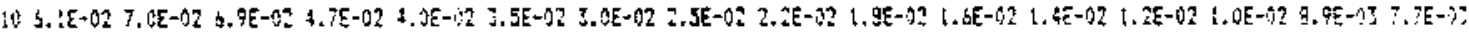

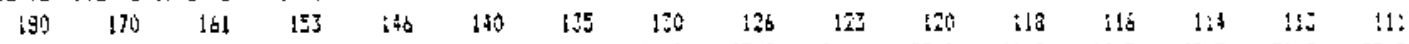

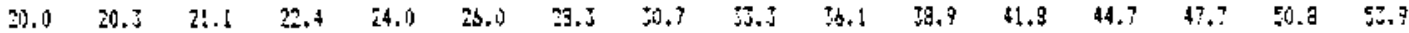

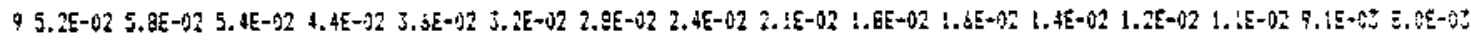

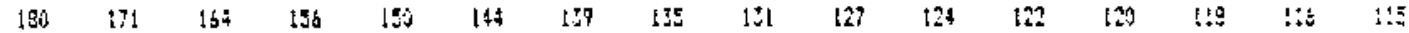

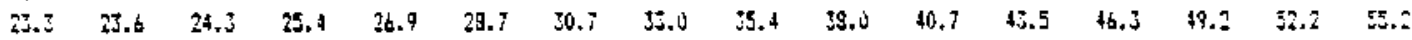

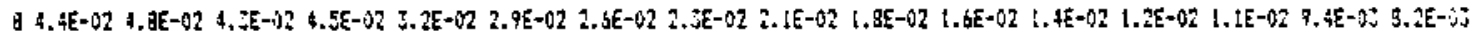
$\begin{array}{lllllllllllllllll}190 & 172 & 165 & 159 & 153 & 147 & 145 & 139 & 135 & 131 & 129 & 12 b & 123 & 121 & 119 & 115\end{array}$

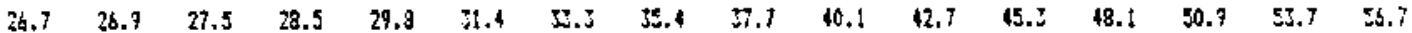

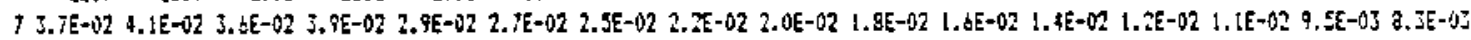

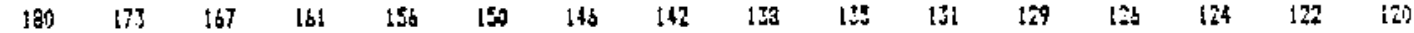

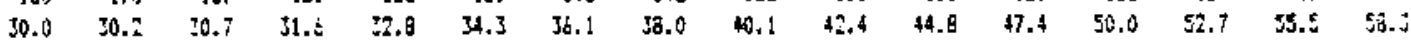

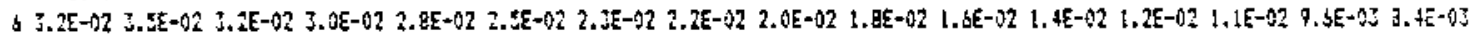

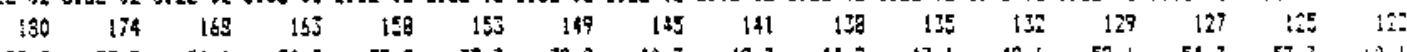

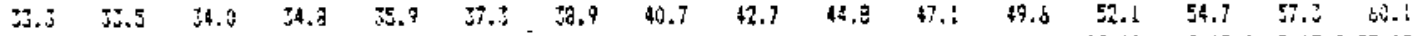

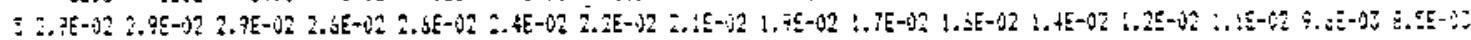

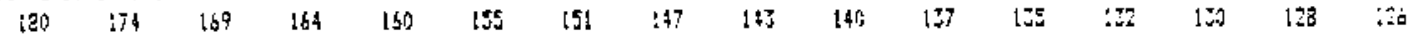

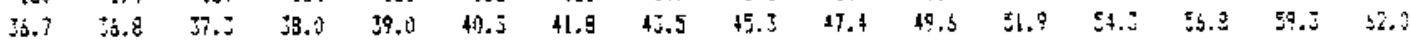

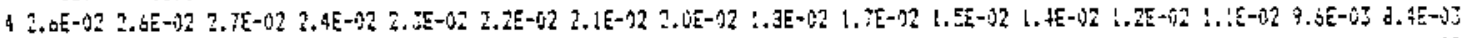
$\begin{array}{lllllllllllllllll}190 & 175 & 170 & 165 & 161 & 157 & 153 & 159 & 146 & 143 & 140 & 137 & 155 & 152 & 130 & : 39\end{array}$

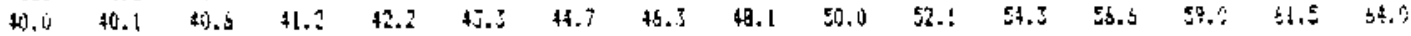

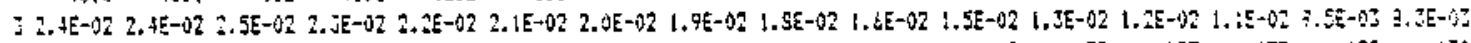

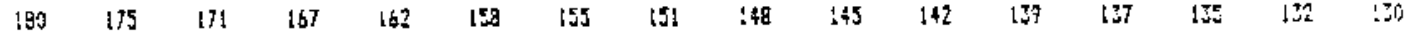

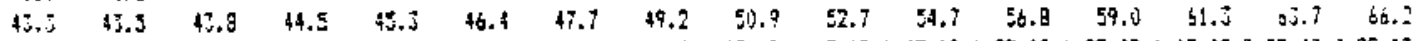

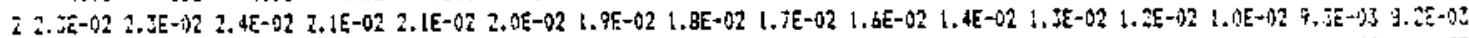

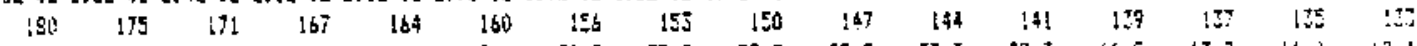

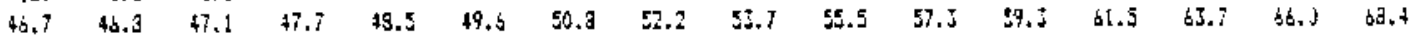

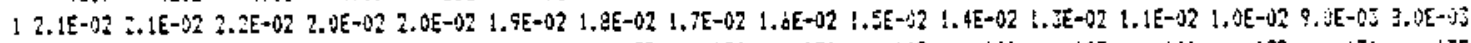

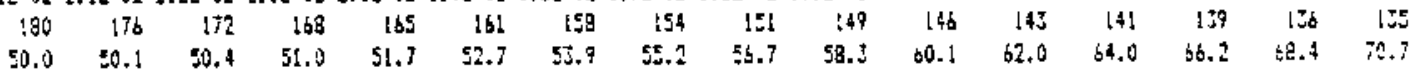

External Dose (rem) from the Plume Estimated for 00002 May 7, 1986, for the Southeast Quadrant. Bearings are in Degrees and Distances are in Miles from Chernobyl. 


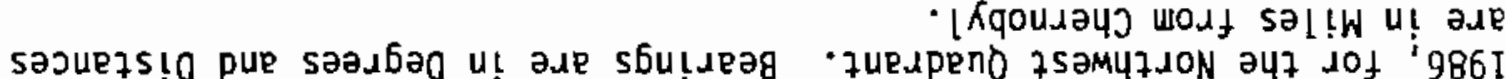

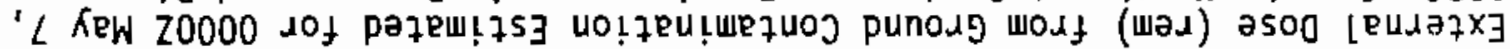

\begin{tabular}{|c|c|c|c|c|c|c|c|c|c|c|c|c|c|c|c|}
\hline 0 & $5 \%$ & $\therefore 9$ & 0.61 & $2 \hat{2} i$ & 1:9! & 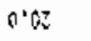 & 58 & $\log _{\pi}$ & 0.05 & 20 & 194 & $0.0 \mathrm{~s}$ & $\therefore$ & 196 & 0.05 \\
\hline c & $O L i$ & $\mathrm{Gii}$ & $0 i i$ & $\theta E$ & $0<z$ & $0 L$ & $0 / 2$ & $0 \leqslant 2$ & $0 \leqslant$ & WLt & $G L$ & $\theta C_{i}$ & ofiz & $O L Z$ & 012 \\
\hline $1+3 I V$ & $00+36=2$ & $00+36 \%$ & $0,47^{\circ} \cdot 1$ & {$[0-30 \%$} & $10-31 \%$ & $10-31+5$ & $10-32 \div 5$ & $10-36=$ & $10-39 \cdot 9$ & $10-39 \%$ & $10-33 \times 1$ & $10-3 \approx 1$ & $10-3 i+i$ & {$[0-30 \cdots$} & $20-3^{3} \cdot 6$ \\
\hline$\because$ & Ir & $5 \cdot 1$ & 500 t & 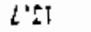 & 0.11 & $50 ?$ & $92 \pi$ & 692 & $2 * 0$ & $s, \underline{s}$ & $E^{\circ} 95$ & $100 t$ & S.St & $8^{\prime} 9 t$ & 100 \\
\hline & SiE & 962 & 882 & 452 & I I ב & 617 & $B Q Z$ & Lii & $92 \pi$ & $i 2$ & ${ }_{s t z}$ & $H L$ & 12 & $b i z$ & 21 \\
\hline $39 \div$ & $10+75 \%$ & $00+52=0$ & $6 \hat{6}+3 S^{2}$ & $n \hat{n}, \pm=1$ & $0 n+3: 1$ & $10-95=8$ & $10-3 b y$ & $10-3 b^{\circ} 2$ & $10-30 \%$ & $10-3 E^{2} \mid$ & $10-3 y^{\prime} 1$ & $10-350 i$ & $10-5 i+1$ & $16-3111$ & $20-3 i a$ \\
\hline :c & 34 & $t^{2} 6$ & 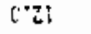 & $t+1$ & OEI & $1 \cdot 12$ & 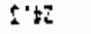 & $52 i$ & $10 \%$ & $i^{\prime}+1$ & $\because t s$ & $9+0 t$ & ESt & $T / t$ & 103 \\
\hline 0 & sis & sif & $20 \div$ & $93 i$ & 167 & 682 & sat & 19 & $\tau E i$ & $19 i$ & $08 i$ & $t \leq 2$ & 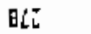 & $8 i i$ & $L L$ \\
\hline $0+32 \div 2$ & $00+36=2$ & $00+36=z$ & $10-3 \div-8$ & $10-3 t^{2}$ & $n+3=1$ & T & $10-3[2 \mathrm{~g}$ & $10-3112$ & $10+36.1$ & $10-30$ & $|0-3| z$ & $10-35 \cdot 1$ & $10-37 * 1$ & $10-3 i \%$ & {$\left[n-3 T^{+} !\right.$} \\
\hline $0.0 !$ & $50 !$ & $0 \% !$ & ;'t & . 91 & 5.61 & 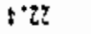 & $f=s z$ & $\Xi^{*} \theta ?$ & $9^{\prime} \sqrt{2}$ & $8^{\circ} \mathrm{t}=$ & $\hat{i} \cdot 8 i$ & $z+1 t$ & $5.6 t$ & $t \cdot 2 t$ & 0.15 \\
\hline (1) & $1 t=$ & $92 ?$ & sis & 908 & $n_{1} t_{0}^{2}$ & 952 & ctt & 062 & $8 Q_{i}^{*}$ & PG: & $58 i$ & HEE & 28 & 28 & $t E i$ \\
\hline$+3 E^{\prime} 1$ & $00+3=1$ & $00+3 x^{2} \mathrm{C}$ & $00+3+1 !$ & $10-3 t \cdot r$ & $10-36^{0} \div$ & $\mid \hat{v}=3 t+1$ & 1 & $001+3 ;)^{\circ}$ & $10-30)$ & $10-39+9$ & $16-3 B^{\circ}$ & IO-Y & $10-3: 2$ & {$[0-3 i *]$} & $10-j \mathrm{r} ;$ \\
\hline ! & {$[?]$} & 6010 & $1.9 i$ & 6ा & 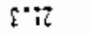 & car & $b^{2} \varphi^{2}$ & $\varepsilon^{\prime} \in z$ & $8 \cdot 2 \hat{z}$ & $b \Gamma$ & ris & $2 \cdots$ & $\tilde{2}>\mathrm{st}$ & $5^{\circ} 8 t$ & โרa \\
\hline & $\hat{3}+\hat{L}$ & as & $\pi:$ & sit: & $20 \Omega$ & $\cos$ & $b 6 \bar{z}$ & 962 & 85 & 162 & $\Delta Q \vec{i}$ & 852 & $\angle 8 i$ & 82 & at \\
\hline i & $\because i 1-3 i: t$ & $0,0+391$ & $0 i+3 t-1$ & $10-3.2$ & $10-3922$ & $10-3601$ & $10-3: 21$ & $10-3 \varepsilon \cdot z$ & $6-35 \cdot 5$ & $1\left(1-3 E^{*}\right.$ & $\mid[x-3 \mid \delta$ & $10+31 \cdot 2$ & $\mid Q-3,-5$ & $10-3 t \cdot 2$ & $16-3 !$ \\
\hline in & 0.21 & :L': & 5.61 & $\hat{i} \cdot \tau$ & $9 \cdot \llbracket z$ & 0.92 & $l^{\prime} B z$ & ris & s't? & 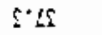 & 5 & s.s & $19 \%$ & 9.65 & 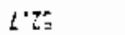 \\
\hline & $81 \%$ & 825 & ber & in & 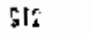 & $00 \Omega$ & 595 & $2 \sqrt{2}$ & 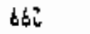 & 902 & $\$ 02$ & $E t z$ & bac & 632 & $88 \mathrm{i}$ \\
\hline$(-3:-2$ & IR-3n's & $10-3505$ & $\left(0,0+30^{\circ}\right]$ & $10-51 \%$ & $15-3 j=9$ & $10-36.1$ & {$[n-3+\cdots$} & 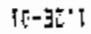 & $10-321$ & $10-312$ & $10-3 \div 4$ & $10-3044$ & $10-3 t \cdot 1$ & $10-3 \hat{\varepsilon} \times 9$ & $10-39:$ \\
\hline o & 502 & t'i? & $7 z$ & 0.62 & $0: 0 \%$ & 528 & los & $2 n$ & 198 & $6.8 i$ & $B^{\circ} 14$ & t'tt & $t \cdot t r$ & $0^{\circ} 05$ & $6 \%$ \\
\hline J & $0 \pi$ & 185 & $\mathfrak{s}$ & qZ: & $0 z i$ & SIt & ors & $90 \hat{\imath}$ & Cot & $00 \%$ & $\partial b^{2}$ & $56 z$ & 706 & 264 & 166 \\
\hline $0-35 \div 5$ & $10-36.5$ & $10-32 \cdot 4$ & $10-36=9$ & $10-30 \% 6$ & $10-3 \times 1$ & $1 c-3 t^{\circ}$ & $10-30 \div ?$ & ז' & $20-30 \%$ & $20-3 t+6$ & $10-51.1$ & $10-36.1$ & $10-3015$ & $10-31+1$ & $10-3 \Sigma^{\prime}$ \\
\hline 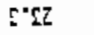 & zos & 5 & 10 & 6.92 & t $8 z$ & $t^{\circ} 0_{2}^{n}$ & $\mathrm{BO}^{\circ} \mathrm{EI}$ & r.SS & 0.65 & $10 \%$ & $s \cdot t$ & $\mathfrak{\varphi}$ & I'bt & $\because \pi$ & $2 \mathrm{KS}$ \\
\hline & IS & this & PIf & OS: & bir & 6Is & SIt̂́ & IIi & 105 & $10 / 2$ & 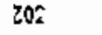 & wo. & 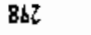 & 200 & 0 \\
\hline "? & $10-30 \%$ & $10-30^{\circ}+$ & โ & $10-37 \cdot 1$ & $10-3 ! I^{\circ} 9$ & $10-3179$ & $10-35 ?$ & $10-3]^{*} 1$ & $20 \cdot 3 t \div 6$ & $20-39 \cdot 8$ & $20-31 \cdot 8$ & $20-3+6$ & $20-3 S^{2} 6$ & $10-3+1$ & $10-31 \%$ \\
\hline$\therefore 9$ & $1-97$ & s'th & S'晒 & $0^{\circ} 6 z$ & 118 & 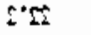 & $+\hat{x}$ & $l \cdot \hat{\mathfrak{s}}$ & 106 & $2 t$ & S\$ & 㷖 & 6.05 & $l \approx \hat{s}$ & $l^{\prime} \xi_{3}$ \\
\hline & $2 s$ & St: & $6 \Omega 5$ & sit & $t z \xi$ & str & Bt? & 512 & II & 805 & 905 & EOS & 105 & 662 & $80^{\circ}$ \\
\hline $10-30 \div$ & $10-33 \% 2$ & $10-3+8$ & $16-3518$ & $10-362$ & $10-3 \div \div 4$ & 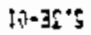 & $10-36 \times 5$ & $10-3 \Sigma^{2} z$ & $2 n-30 \%$ & $20-3 ; * 8$ & $20-3 z_{3}^{*} t$ & $20-30.2$ & $20-3: 9$ & $20-30 \div 2$ & $2:-3 x^{r}:$ \\
\hline w & $i \cdots$ & $\therefore 0 \Omega$ & 915 & $E^{\prime} Z_{i}$ & $r r^{\prime}$ & I' & $a^{2} B r$ & $i^{*} 0 t$ & $t$ & $\varepsilon$ & $r t t$ & 6.45s & 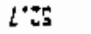 & 535 & $58 \%$ \\
\hline 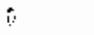 & $2 \mathrm{si}$ & It: & 15 & 98 & $n$ & $9 \mathrm{~K}$ & $z ?$ & $B F_{L}$ & $\sin$ & ii: & tof & 90 & bet: & $20:$ & tro: \\
\hline$n-3+1$ & $t \hat{0}+3=2$ & {$[0-3\} \div$} & $r 0-3 t \cdot z$ & I I & $: 0-30: 2$ & $k-3 t+s$ & $10-3)^{\prime} \mathrm{k}$ & $10-30^{\prime} \mathrm{k}$ & $10.366^{\circ} i$ & $2 n-3 \div=8$ & $21-3 B^{\prime} q$ & $20-3 i \cdot 9$ & $20-31 \% \mathrm{G}$ & $25=35 \% 5$ & $20-0$ \\
\hline$\because \hat{\imath}$ & $\because 2$ & Qta & $E$ & $\mathrm{j}^{\prime} \mathrm{si}_{\mathrm{j}}$ & $\because n$ & $\therefore \varepsilon_{i}$ & $t .0 t$ & $t$ & $E$ & $i t s$ & $\hat{\imath}$ & I'T; & In & $\because 3$ & $\because$ \\
\hline & tit & Et $\hat{\mathbf{L}}$ & $\$ \neq \hat{\imath}$ & $8 \%$ & 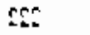 & Dis & S: & $i z$ & 81. & sis & it: & $0 . v_{2}$ & 10 & 508 & $\sin$ \\
\hline $20-360^{\circ}$ & $10-360$ & $10-3 P^{\prime} 2$ & $10-3572$ & $\mid t j-3 t^{\prime} z$ & $10-3 i \cdot 2$ & $\eta(1-3) \cdot z$ & $30-32{ }^{2} k$ & $16-305$ & $|6-3|^{\circ} \mathrm{i}$ & $10-3 \div 11$ & $20-350$ & $20-35 \times 5$ & $2 \hat{b}-3 t$ & $20-3 E^{2}+$ & $20-36$ \\
\hline$t$ l'og & $E^{\prime} 9 \hat{0}$ & S'ti & $0^{2} \varepsilon \hat{\alpha}$ & t. $6 \hat{:}$ & Sot & E't & $s+5$ & ו 12 & $t ? t$ & 9.64 & 615 & E' $k 5$ & 9.95 & $\{.65$ & B. 5 \\
\hline 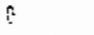 & 5 & $6 t: 2$ & the & at: & $5 \approx$ & $\ln$ & LIs & 282 & atr & tis & sis & 215 & is & ac: & 90 \\
\hline$\langle\hat{g}-3 \hat{\xi}=0$ & $\mid \hat{v}-3 z+1$ & $10-36.1$ & $10-3 t^{\prime} z$ & $10-3 i 2$ & $16 \times 30^{\prime} 2$ & $i b-3 t+i$ & $10-3 i<\varepsilon$ & $10-3: '\}$ & $10-36$, & $0 \%$ & 30.1 & & & & 35 \\
\hline 0 & I'ot & $90 \%$ & int & i'z & 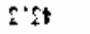 & $t " b t$ & $\because 9 p$ & $I^{\prime} \mathrm{zt}$ & 0.05 & 125 & $\therefore 5$ & 893 & 0.55 & 5019 & 0.19 \\
\hline & ss: & $0: \hat{2}$ & St5 & $1 \%$ & an & 25 & $62 \xi$ & 425 & $5 \pi$ & Q27 & :Is & sits & 11 & 015 & 805 \\
\hline$(4-30.5$ & $2(x-3) \cdot 1$ & $10-3 t \cdot 1$ & $10-30 \cdot 2$ & $10-\geq 27$ & $14-30$ & {$[\{* 3 t \cdot]$} & {$[0-3)^{n}+1$} & $10-37 \times ?$ & $t 5-30 .+$ & $10-25+1$ & $10-3 \div \div$ & $i c-3 i \cdot i$ & $20-39 \%$ & $20-30 \%$ & $20-3$ \\
\hline 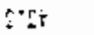 & G.5t & 8 & 5.65 & $\therefore$ & 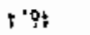 & $12 t t$ & $\tau \prime b t$ & 6.05 & : & $1+3$ & $\tilde{E}\}_{\xi}$ & 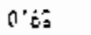 & $\because \because 3$ & $\therefore 3$ & $z 97$ \\
\hline 6 & $\mathrm{sni}$ & ISE & $2 t r$ & $2 t_{i}$ & E:r & $\Omega \Omega$ & In & $E_{z i}^{-i}$ & $50 \hat{z}$ & $z Z^{r}$ & bis & ia & 5 & 21! & $0: 2$ \\
\hline $26-3018$ & $2(1-3)^{*}+\xi$ & $20-3 \pm \cdot 6$ & $10-3 a+1$ & $10-3641$ & $t_{0}-3 ; z$ & {$[C-3 i \cdots$} & $t 0-5 \times 1$ & $10-37^{-1}$ & i $[-3] \cdot z$ & $1-3-3 * \hbar$ & $3 i-30 \%$ & $10-730$ & $z_{G-j i} \cdot q$ & $\left.20-3 a^{\prime}\right)$ & $20-3 \varepsilon^{\circ}$ \\
\hline 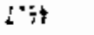 & $E^{\circ} \varphi_{t}$ & $\because t h$ & $L^{\prime \prime}: t$ & s.8t & 854 & $E \times 5$ & E. & ITs & $\bar{n}$ & $\because \Omega$ & $\because 6 \hat{z}$ & $\because 19$ & $1 \% 8$ & $0.9 \%$ & $E$ \\
\hline j & s:- & $\hat{M}$ & $\operatorname{tr}$ & $t \geqslant \varepsilon$ & the & $9 \mathrm{LS}$ & Cis & (1): & $L S$ & tis & {$[3$} & bIf & IIS & GIS & st5 \\
\hline & $20.3: * t$ & $2 i-3 t \cdot 9$ & $|0-3| 1$ & {$[1-39 \cdot 1$} & $\mid\left(6-3 E^{\prime} !\right.$ & $52-3 ! 1$ & $2\{-3\}=1$ & $I C-3: i$ & $\mid c-3 \approx 1$ & $w-3 x=$ & $10-3 \xi c$ & $\mid(1-3)=8$ & $31-3601$ & $20-3: \div 9$ & $20-19+10$ \\
\hline & 103 & 1.03 & 8 is & 243 & $\because \because 3$ & 60 & 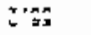 & $\because \varphi_{3}$ & $\because 33$ & $\because$ "lip & $82 ?$ & $C^{\prime} \mathbf{r}$ & 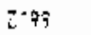 & $P \cdot B 7$ & $t \hat{\varphi_{2}^{\prime}}$ \\
\hline & $9 \hat{2 i}$ & $2 S s$ & $8 \neq:$ & $5+5$ & $i r_{i}^{\prime}$ & $\tilde{a x h}$ & nis & If & $b^{y=}$ & 9:2 & 25 & $i \hat{i} \hat{x}$ & \pm 18 & $91:$ & sit \\
\hline & $2 \div-32 \div$ & $3-3 x+1$ & $20-309$ & the & $\mid i-371$ & $19-3 ! 1$ & $10-35 \div 1$ & Is-is"t & $16=3: \div 1$ & $10-3 ; \div 1$ & $10-35 \cdot z$ & $I n-5+3$ & : & $1 \hat{-3}+1$ & $20-32$ \\
\hline $3:$ & il & $t$ & 1 & $\because:$ & 11 & of & & & 6 & $?$ & ; & $t$ & 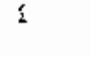 & $i$ & $i$ \\
\hline
\end{tabular}




\begin{tabular}{|c|c|c|c|c|c|c|c|c|c|c|c|c|c|c|c|}
\hline \multicolumn{16}{|c|}{ a corfoinate of the grid peints } \\
\hline 1 & 2 & 3 & ; & $\Sigma$ & s & 7 & $\mathrm{a}$ & 9 & $\because$ & $::$ & i: & $1:$ & 17 & $1-$ & is \\
\hline & & 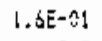 & & & & $E+10$ & 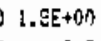 & cat? & $95+30$ & 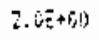 & $75+04$ & $E+C ?$ & $2+3$ & $1 E+x^{2} c$ & 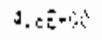 \\
\hline isb & 265 & 265 & 265 & 264 & 264 & 263 & $26 ?$ & $2 b:$ & $2 t \cdot j$ & $2 E_{0}$ & 255 & 751 & 25 & 225 & !E: \\
\hline 50.1 & 46.8 & 43.5 & $\$ 0.1$ & 36.8 & 33.5 & 50.2 & 25.9 & ه.j. & 20.3 & 12.0 & 10.7 & $1 \because \varepsilon$ & & $4 . \overrightarrow{.}$ & \\
\hline$\{E-0 !$ & $3 E-01$ & BE-0? & $4.2 E+1: D$ & $1.25+00$ & $5.0 E+100$ & $1 . \dot{a} E+\hat{v} 0$ & 7. $3 E-\hat{i} 1$ & $1.2 \varepsilon+0 j$ & $1.7 E+015$ & $(1, \boldsymbol{E}+\mathbf{E})\}$ & $1.3 E+40$ & {$[., 4 E+910$} & $1.35+1,3$ & $3, Z E+y 0$ & IE-ily \\
\hline 262 & 261 & $26 !$ & 260 & 259 & 258 & 257 & $25 s$ & 254 & 251 & 248 & 243 & $2 J b$ & 255 & 206 & $\$ 0$ \\
\hline 50.4 & 47.1 & 4.3 & 59.5 & 3.3 & 34.0 & 30.7 & 27.5 & 34.3 & 21.1 & 18.0 & 14.7 & 12.1 & 7.7 & 7.5 & i.? \\
\hline$[E-3 t$ & $5.9 E-0 l$ & $7 E \div 00$ & Pet $P E+60$ & $4.7 E+00$ & $3.1 E+\infty 0$ & $4 \varepsilon-31$ & $5.35=0.01$ & $\Delta E-0 t$ & $5,8 E-01$ & $0 . \$ E-01$ & $9 . \hat{z} E-0 t$ & $1.2 \mathrm{E}+1) \mathrm{U}$ & A.JE+00 & $2.15+100$ & Eforit: \\
\hline 259 & 257 & $\pi$ & 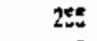 & 254 & 25 & 35 & 249 & $2+6$ & 243 & 377 & 203 & 225 & 213 & 178 & 180 \\
\hline $51 . \hat{5}$ & $\$ .7$ & 44.5 & $\because .2$ & 2.0 & 34.5 & 31.8 & 29.5 & 25.4 & 22.4 & $: 7.4$ & 9.7 & $: 4.1$ & $\therefore$ & 10.5 & $1 \ldots$. \\
\hline$[E+1]$ & $1.75+3,1]$ & $42+100$ & tetult & $E-0 !$ & $3.3 E-01$ & $7 E-01$ & 1 $2.9 E-01$ & $\varepsilon-\emptyset \mid$ & 4. $0 E-0 \mid 01$ & $5.38-3 !$ & $1.15-0,1$ & $1.1 E+10$ & $1.35+8$ & 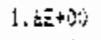 & $! . \Delta E+0)$ \\
\hline 255 & 254 & 252 & 251 & 25i! & 248 & 266 & 243 & 240 & 273 & 271 & $2: 5$ & $2 ! a$ & 200 & 194 & 150 \\
\hline 5.7 & 49.5 & 45,3 & 42.2 & 39.0 & 35.9 & 32.8 & 29.5 & 25.9 & 24.0 & $2 ! . j$ & 15.9 & 16.7 & 14.5 & 15.7 & 18. \\
\hline t & & $=-v$ & $[5-1)]$ & [E-9! & $1.1 E \sim 01$ & $E E-0 !$ & 2. $.0 E-01$ & $2,5 E-01$ & 3. $35-31$ & $4.5 E-0 !$ & a. & $1.2 \mathrm{E}+\infty 0$ & $1.25+80$ & $1.4 E+C O$ & $1.3 E-j i j$ \\
\hline 251 & 250 & 2* & 247 & 245 & 243 & 240 & 257 & $2 \pm t$ & 230 & $2 \hat{k}^{x}$ & 219 & 210 & int & 171 & 180 \\
\hline 52.7 & $\$ 9.6$ & 46.4 & 4.3 .3 & 40.3 & 57.3 & I4.J & 31.4 & 29.7 & 24.0 & 2.5 & 21.3 & 19.4 & $100 \times 2$ & 17.0 & 16.: \\
\hline $45-1\}$ & $2 E=01$ & $2 E-01$ & $2 E-01$ & |. . $E=-0 \mid$ & 1.JE -1$) !$ & $1.4 E-0 !$ & $7 E-01$ & 2. ZE-DI & $2 . \Delta E-01$ & 2. $7 E-D 1$ & $3 E-i) !$ & $7.7 \varepsilon-01$ & $G E-41$ & $15+\infty 0$ & $1 E+00$ \\
\hline 8 & 248 & 245 & 243 & 241 & 239 & 255 & 253 & 27 & 225 & 219 & 215 & 206 & 198 & 103 & 100 \\
\hline 50.9 & 50.9 & 1.7 & 44.7 & 41.3 & 8.9 & $2 b .1$ & 3.3 & 34.7 & 23.3 & 26.0 & $24.1)$ & 22.4 & 21.8 & 0.3 & 20.0 \\
\hline $2 E-8$ & $9.3 E-62$ & $E=-12$ & bE- $v_{2}$ & $E-01$ & $\varepsilon=01$ & JE-0! & 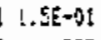 & $9 E-01$ & 2.5E-01 & 4. $25=0 !$ & 6.jE-jt & qE-01 & $1 E-01$ & $05-01$ & END! \\
\hline 244 & 243 & 211 & 239 & 23 & $x$ & 232 & 38 & 20 & 220 & 315 & 209 & 203 & 19 & 198 & 150 \\
\hline 55.7 & 52.2 & 49.2 & +6.3 & 43.5 & 40.7 & 30.0 & $3 \pm .4$ & $I .0$ & 30.7 & 29.7 & 36.9 & 25.4 & $24 .$. & 3.5 & 20.5 \\
\hline-1$) 2$ & $E=0$ & {$[-02$} & $E-1$ & $E-42$ & 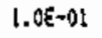 & 1 & $E-01$ & 91 & $\mid(-1)]$ & $2 E-01$ & gE- $0 t$ & $4 E-01$ & $1 E-0 !$ & $\mathrm{FE}-\mathrm{H} !$ & $65-01$ \\
\hline 2 & 240 & 278 & 238 & 233 & 231 & 28 & 225 & $22 !$ & 216 & 212 & 206 & 200 & 194 & 197 & $1 B C$ \\
\hline 5.7 & 58.7 & 30.9 & 18.1 & 4 & 42 & to.l & 3.7 & 50.4 & $\pi .3$ & $\$ 1.1$ & 29.8 & 29.5 & 27.5 & 26.9 & $26 . I$ \\
\hline $\mathrm{J}$ & $E-02$ & 2 & $k=02$ & $E=-02$ & $E=01$ & $\varepsilon=01$ & $4 E-0 t$ & $T E-0 \mid$ & 2.5t $=01$ & $F E-01$ & 5.AE-OU & $8 E-1)$ & $a-\sqrt{2}$ & $E=01$ & SE-O1 \\
\hline 259 & 237 & 23 & 28 & & 8 & 235 & 221 & 217 & $\because 3$ & 769 & 203 & 199 & 192 & $18 \delta$ & 180 \\
\hline $5 B .3$ & 25.5 & 32.7 & 30.0 & $\$ 7.4$ & 44,7 & 42.4 & $\$ 0.1$ & 58.0 & 36.1 & 34.5 & 32.8 & 21.6 & 50.7 & 70.2 & 53.6 \\
\hline$t=-9$ & $E=02$ & $4 E-02$ & IE- 02 & $E E-02$ & $0 E=01$ & $E-01$ & CE-0! & $\mathrm{SE}=0$ ! & $1.9 \mathrm{E}-0 \mathrm{t}$ & 2. $|E-0|$ & $5-11$ & J. ${ }^{n} \mathrm{E}-0 !$ & $E-4$ & $E-91$ & $A E=01$ \\
\hline 236 & 234 & $2 \pi$ & 730 & 227 & I25 & $22:$ & 213 & 214 & 210 & 306 & 201 & $!$ & 191 & 195 & 180 \\
\hline$\infty .1$ & 57.3 & 4.7 & 52.1 & 49.6 & 1 & 44.3 & 42.7 & 40.7 & 58.9 & 37.3 & 35.9 & {$[A, 9$} & 34.17 & 5.5 & 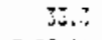 \\
\hline & $=-t$ & 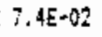 & $i$ & $E-14$ & $E-01$ & $1 E-01$ & ! ! 范- & $E-01$ & $7 E-01$ & $E-\Delta 1$ & $2.2 E-31$ & $95-131$ & E-c! & : $:-1 !$ & 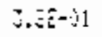 \\
\hline 23 & $\pi:$ & 22 & 2 & 2 & $2 m 2$ & 213 & $2: 5$ & $2 ! 2$ & 208 & 204 & $: 99$ & 195 & 100 & 155 & $18 ;$ \\
\hline b2 & 59.5 & 56.8 & 54.3 & 51.9 & 49.6 & 47.4 & 45.5 & 4.5 & 41.3 & 46.3 & 37.2 & 38.3 & 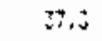 & 3. & 8.7 \\
\hline & & 0 & 19 & $E-02$ & $E-02$ & $E-01$ & $E-0$ ! & $E-9 !$ & $(E-0)$ & $1.35-\Omega 1$ & 2.0E-ओ & $2.3 E-111$ & $=.4 E-8 !$ & $2.9 E-B S$ & 3.ZE-?1 \\
\hline 23 & 224 & 2 & & $\therefore$ & 19 & a & 213 & 210 & 206 & 202 & 198 & 194 & 159 & 194 & $18 ?$ \\
\hline $34 . j$ & $6 ! .5$ & 89.0 & 56.6 & 54.3 & $52 .:$ & 50.9 & 46.1 & 44.3 & 4.7 & 4.8 & 42.2 & 41.2 & 40.6 & so.: & $\$ 9 . j$ \\
\hline$\angle E-\Delta 2$ & $7 E-62$ & $3 E-02$ & VE-02 & $85-02$ & $7 E-02$ & E $E-01$ & $2 E-01$ & 5 & SE- -0 & $E=91$ & $1.8 E-6 \mathrm{t}$ & $t-01$ & $3 E-9 !$ & bE-11 & $\Delta[-1) !$ \\
\hline 329 & 22 & 22 & 2 & 220 & 17 & 214 & 211 & 298 & 204 & 291 & 197 & 192 & 188 & $i \approx$ & 180 \\
\hline 56.2 & 65.7 & 01. & 59.0 & 56.9 & 54.7 & 52.7 & 50.9 & 49.2 & 47.7 & 46.4 & 45.5 & 44.5 & 43.8 & 5.5 & $4 \div, 3$ \\
\hline & $1=$ & $j E-1 / 2$ & 5 & $i E-102$ & $E E-62$ & $\{E-\hat{A} !$ & $1 . \pm 5-96$ & $\mathrm{t} t-\mathrm{j} l$ & 1. $4 E-01$ & 1..ะล & 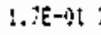 & $2.5 \Omega-1) !$ & C.45-0! & 2.JE-'!! & 2.:E E-1)! \\
\hline$\because$ & $\because 35$ & 2 & 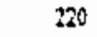 & & 215 & .12 & 209 & 206 & 203 & 197 & 195 & 192 & 199 & 184 & 18 \\
\hline tQ. & 66.0 & 63.7 & 81.5 & 59.5 & 53.3 & 55.5 & 53.7 & 52.2 & 50.9 & 49.2 & 49.5 & ti.? & $47 .:$ & +6.3 & 46.7 \\
\hline 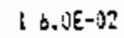 & $\varepsilon-02$ & $1 E-02$ & $B E-\theta 2$ & ₹E-12 & $\varepsilon-02$ & $D E-01$ & $1 E=0 t$ & IE-0t & HE-01 & $E \varepsilon-01$ & $6 E-01$ & $E-\$ 1$ & $0 E-n !$ & JE- & . $(E-1) t$ \\
\hline 225 & 223 & 220 & 218 & $2 ! 6$ & $\dot{x}$ & 210 & 98 & 205 & 201 & 198 & 194 & 99 & 187 & 132 & iec \\
\hline 70.7 & 63.4 & 6.2 & 64.0 & 2.0 & 60.1 & 59.3 & 56.7 & 55.2 & 53.9 & 52.7 & 51.1 & 51.0 & 50.4 & E.:! & 50.0 \\
\hline
\end{tabular}

External Dose (rem) from Ground Contamination Estimated for $0000 Z$ May 7, 1986, for the Southwest Quadrant. Bearings are in Degrees and Distances are in Miles from Chernobyl. 


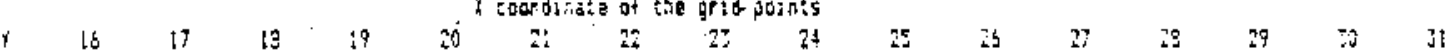

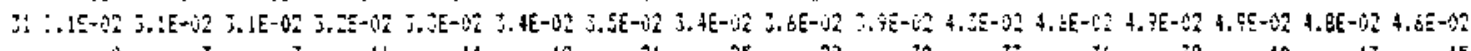

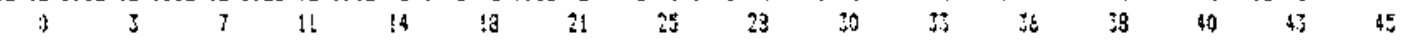

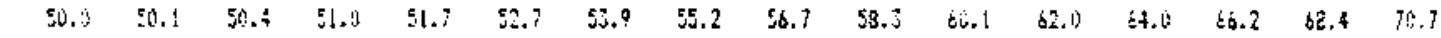

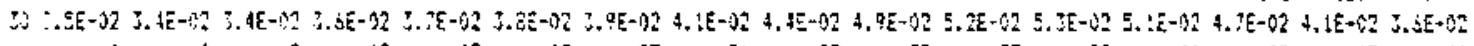

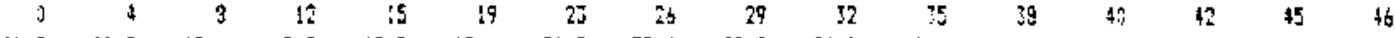

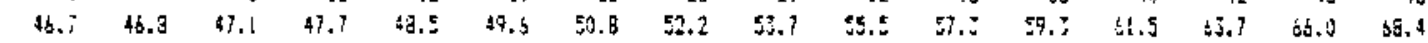

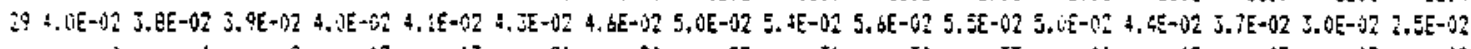

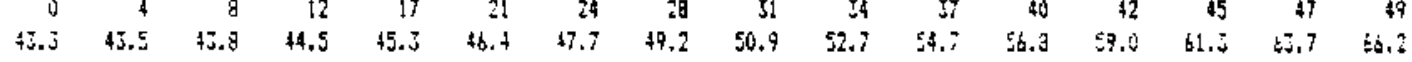

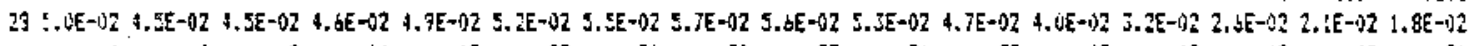

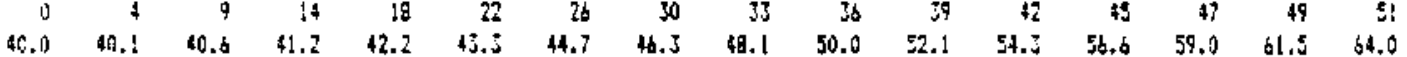

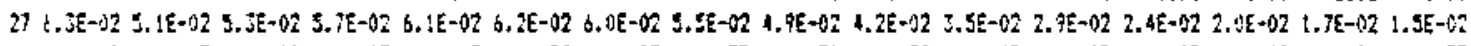

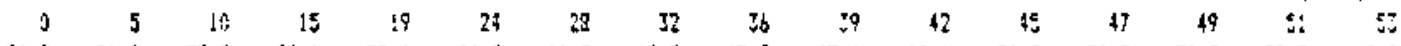

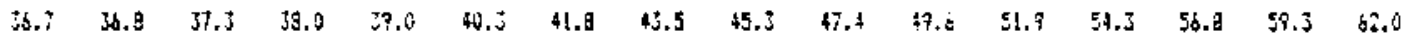

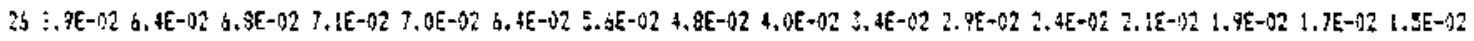

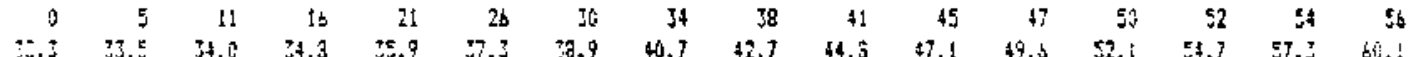

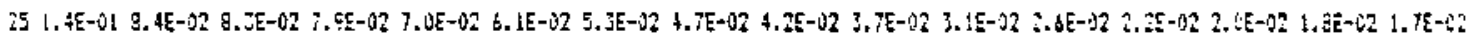
$\begin{array}{llllllllllllllll}0 & 6 & 12 & 19 & 23 & 29 & 3 & 37 & 4 & 45 & 48 & 59 & 53 & 5 & 57 & 57\end{array}$

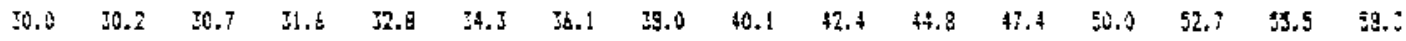

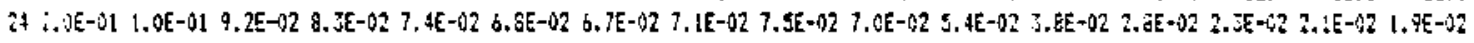

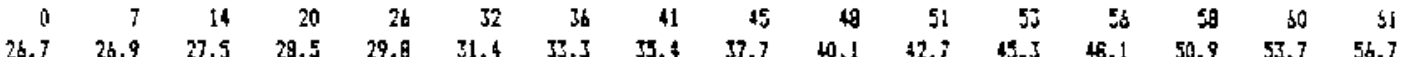

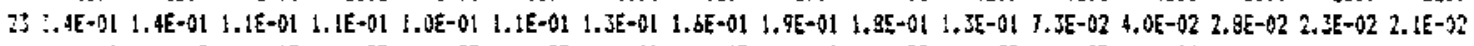

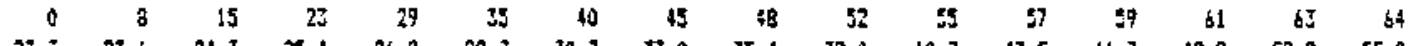

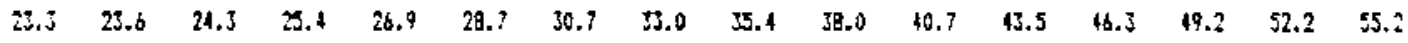

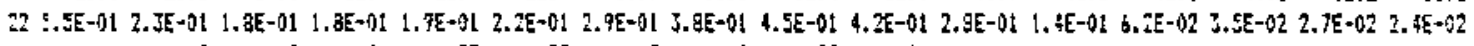

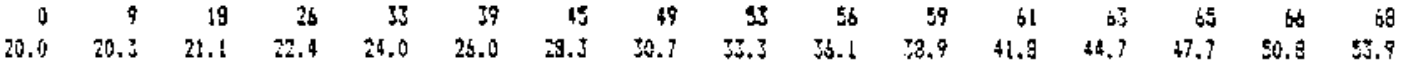

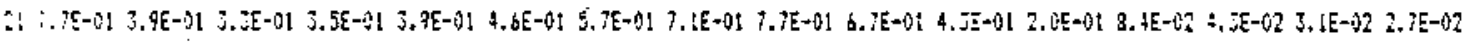

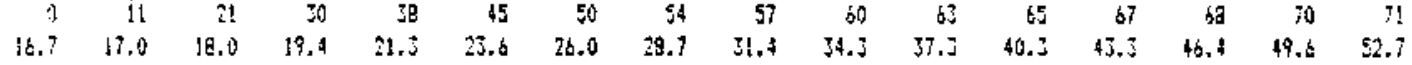

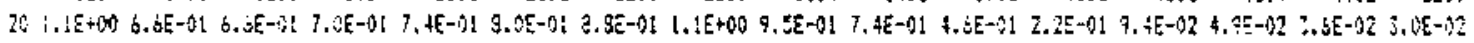

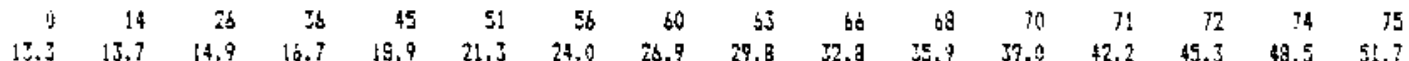

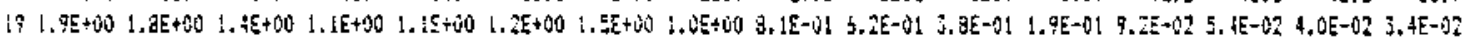

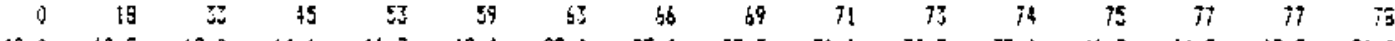

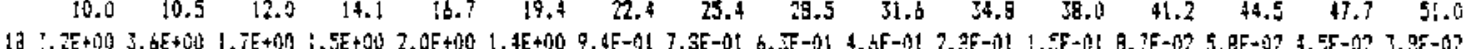

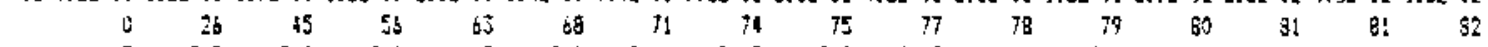
$\begin{array}{llllllllllllllll}0.7 & 7.5 & 9.4 & 12.0 & 14.9 & 13.0 & 21.1 & 24.3 & 27.4 & 50.7 & 34.0 & 37.5 & 40.6 & 43.3 & 47.1 & 50.4\end{array}$

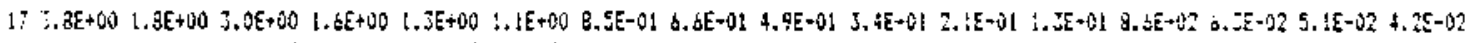

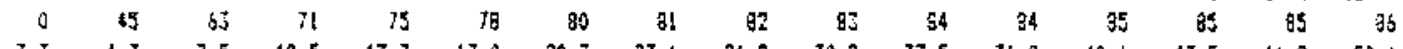

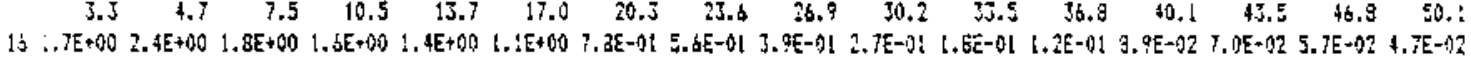

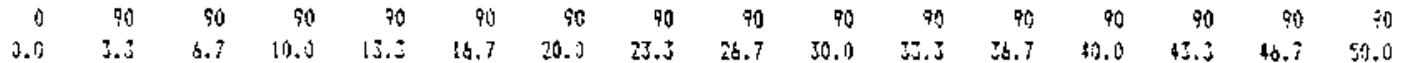

External Dose (rem) from Ground Contamination Estimated for $0000 \mathrm{Z}$ May 7 , 1986, for the Northeast Quadrant. Bearings are in Degrees and Distances are in Miles from Chernobyl. 


\begin{tabular}{|c|c|c|c|c|c|c|c|c|c|c|c|c|c|c|c|}
\hline & & & & & i acors & ate 0 & grid & 010 s & & & & & & & \\
\hline ts & $1 i$ & : & $\because$ & $\therefore$ & 3 & $\ddot{z}$ & $Z$ & 24 & $\therefore$ & 23 & $\because$ & 29 & $\therefore$ & $3 i$ & $\because:$ \\
\hline & & & & & & & & & & & & & & & \\
\hline$: 30$ & 135 & $1: 5$ & 1.29 & $i 24$ & 101 & $\varphi 9$ & 99 & 97 & $9 b$ & 75 & 75 & 94 & 94 & 9.4 & $\because$ \\
\hline i..j & 4.7 & 7.5 & 0.0 & 12.7 & 17.0 & 80.3 & 23.6 & 26.9 & 50.2 & 5.5.5 & 36.8 & $4 i .1$ & 4.5 & +6.3 & 滋1 \\
\hline+0 & $1.75+10$ & $E+i$ & $9 E+1)$ & $2 E+00$ & $5-0 !$ & $3 E-1)$ & $E-0$ & $t z-0 !$ & ; & $\mid E-1) \mid$ & $(2 E-0) !$ & OE $-i$ & $4 E+62$ & FE- 02 & 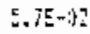 \\
\hline$! 80$ & $15 \mathrm{~J}$ & 175 & $12 \mathrm{~J}$ & 116 & 111 & 108 & 105 & 104 & 102 & $10 !$ & 160 & 79 & 98 & 汨 & $3 i$ \\
\hline 3.: : & 7.5 & 9.4 & (2.1) & 14.9 & 18.9 & 21.1 & 24.3 & 27.5 & $39 . ?$ & 34.0 & $5 \pi .3$ & 40.5 & $4: .8$ & 17.! & 50.7 \\
\hline$\partial E+\infty \theta$ & $2.5 \bar{z}+60$ & $1.6 E+00$ & $1.5 E+90$ & $1.0 E+00$ & . $3 E-31$ & $5.7 E=01$ & 4. IE-G1 & S. HE-OI & $2.3 \Sigma-111$ & 1.9E-3! & 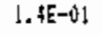 & $1, ! E-t) !$ & $9.1 E-122$ & $\sqrt{2}-72$ & :[E-4]? \\
\hline 180 & 161 & 190 & 123 & $12 b$ & [2] & 115 & 113 & 110 & !96 & 105 & 105 & 104 & 102 & 102 & 121 \\
\hline 10.0 & 10.5 & 12.0 & 14.1 & 16.7 & 19.4 & 22.4 & 25.4 & 29.5 & 31.4 & 34.3 & 39.13 & 41.2 & $1+.5$ & 47.7 & 5.2 \\
\hline (. $.5 E+1) 0$ & $2.1 E+\infty 0$ & $|0.4 E+i| j \mid$ & $1.1 \leqslant+00$ & $8,9 E=0 !$ & $9 E-01$ & ક.2飞-01 & $3.4 E+01$ & 3.UE-96 & 2. & {$[. \mathrm{I} E-1)$} & $1.5 E-1) !$ & $1.2 E-01$ & $7,7 \mathrm{E}-1) 2$ & $3 . A E-D 2$ & t: \\
\hline 150 & lbs & 155 & 14 & $1 \leqslant \pi$ & 128 & 123 & 119 & 112 & 112 & 111 & 109 & 108 & 107 & ids & int \\
\hline $1+2$ & [3. 7 & 14.7 & 16.7 & 19.9 & 21.3 & 24.0 & 26.9 & 29.9 & 52.3 & צE.9 & 39.0 & 42.2 & 45.2 & 13.5 & $5 . .7$ \\
\hline$t=t+1$ & 7 cro & (c) & {$[-2]$} & $6-1$ & $t-0 t$ & $75=01$ & 3. $7 E-6 !$ & $B E=0$ & 2. उE-2! & $1,95-0 !$ & 1.5E-41 & $2 x-51$ & $O E-\Delta$ & $45-\sqrt{42}$ & $35-192$ \\
\hline เสอ & 168 & 158 & 149 & 141 & 175 & 129 & 125 & 122 & 119 & :ts & 1.14 & $1: 2$ & 111 & 109 & 168 \\
\hline 16.7 & 17.0 & 19.0 & 19,4 & 21.3 & 23.6 & 26.0 & $29 . i$ & In. & 74.3 & 37.3 & 49.3 & $4 . . j$ & 16.7 & 47.0 & 5.7 \\
\hline $1=+130$ & $\therefore .3 E+00$ & $4 E+10$ & 7. $\mathrm{gE}-1 \mathrm{i})$ & 6. $4 E-0 !$ & 5. JE-0) & $4, J E-0 !$ & $3.5 E=01$ & $2.8 \mathrm{E}-\mathrm{dl}$ & $\{, \pi E-01$ & $1.92-1) !$ & $1,+E-0 !$ & $1.3 E-01$ & $15-9 !$ & $8.3 E-02$ & $3 E-1: 2$ \\
\hline 180 & 170 & $|5|$ & 153 & $1+6$ & $1+0$ & 12 & 130 & 126 & 123 & 120 & 118 & 190 & 114 & 112 & $11 !$ \\
\hline 20.6 & 20.3 & $2 t .1$ & 22.4 & 24.02 & 26.0 & 28.3 & 20.7 & 33.2 & 治, l & $\stackrel{5.7}{*}$ & 11.8 & 44.7 & 47.7 & 50.3 & 5.9 \\
\hline$E=-1$ & $0 F+50$ & S. LE-01 & $2.2 \mathrm{E}-01$ & $d E-0 t$ & $4.6 \mathrm{E}-0 \mathrm{l}$ & $9 E-01$ & $\mathrm{iE}-0 \mathrm{l}$ & $2.7 E-01$ & $2.3 E-0 !$ & $95-101$ & SE- & $3 E-01$ & !E-UI! & . & . \\
\hline نئ & $17 !$ & 1Et & 156 & isw & 144 & 187 & 13 & 121 & 127 & 124 & $! 22$ & 120 & {$[4]$} & 116 & $: 13$ \\
\hline 2.5 & 23.6 & 24.5 & 24.7 & 26.9 & :E.7 & 50.7 & 33.0 & 35.4 & 23.0 & $4 \%$. & 43.5 & $46 . Z$ & 49.2 & 52.2 & $55 . ?$ \\
\hline$x-0 !$ & $\mid[-6]$ & $E-1)$ & $2 x-121$ & $\delta \varepsilon-0 !$ & E- & 1 & UE-0I & $S E-$ & $2 E-0 t$ & $9 E-01$ & SE & $3 E-01$ & $\mid[\varepsilon-0 \mid$ & $3 E-121$ & $B E-52$ \\
\hline 180 & 172 & 165 & 159 & 153 & $1+7$ & 143 & 138 & 135 & 131 & 128 & $12 b$ & 123 & 121 & 119 & 198 \\
\hline 26.7 & 26.9 & 27.5 & 29.5 & 29.8 & $3 !, 4$ & נj.3 & 5.4 & 57.7 & $40 .:$ & 42.7 & 45.3 & 48.1 & 50.9 & 83.7 & 56.7 \\
\hline$[-1]$ & $15-01$ & $\xi-3 !$ & $\{E-0 \mathrm{i}$ & $\vec{j} \varepsilon-\theta$ & 3. $\mathrm{BE}-0 \mathrm{O}$ & $3.2 E-01$ & de-01 & $5[-01$ & $1 E-01$ & $B E-J 1$ & $6 E-01$ & उE-จ1 & {$\left[E^{-1} \mid 1\right]$} & $5 E-122$ & อ. $0 \epsilon-02$ \\
\hline 200 & it & $1 \$ 7$ & $16 !$ & 1.56 & 100 & 16 & 142 & 130 & $1 \pi 5$ & [1] & 129 & $12 \mathrm{~b}$ & 127 & 122 & 123 \\
\hline 30.0 & 30.2 & 30.7 & 31.6 & 32.8 & 34.3 & 36.1 & 58.9 & 40.1 & 42.4 & 44.9 & 4.4 & 50.0 & 52.7 & 55.5 & 50.3 \\
\hline$(E-0):$ & (E-1)! & 4. .5E-6! & 4. $Z E-01$ & $t E-0 t$ & $3.2 E-\uparrow 1$ & $2.9 E-01$ & $2.8 E=011$ & 2.JE-1!! & $2.15-01$ & $1.8 E+0 !$ & $1 . \leq \Im-n !$ & $1.3 E-01$ & $1.1 \mathrm{E}-0 !$ & 9.5E-1) & $3.18 E-02$ \\
\hline 10 & 174 & 169 & $1 \in j$ & $: \leq 5$ & 153 & 149 & 195 & $14 !$ & $1: 3$ & 135 & 102 & 129 & $: 27$ & $1=5$ & 193 \\
\hline 5.7 & ن.3.5 & 34.0 & 34.9 & 75.9 & 37.2 & 53.9 & $\$ 0.7$ & 12.7 & 34.3 & 17.1 & 49.6 & 52.1 & 54.7 & 57.2 & $50 .:$ \\
\hline aE -0 & eE-Sit & -6 & 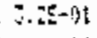 & $3 \mathrm{E}-0$ & $9 E-01$ & 7. $. \tau-01$ & $4 E-191$ & 2. $z E-0 !$ & $05-01$ & $T E-01$ & EE-i]! & $\therefore=25-01$ & !. EE-U! & $3,5=-02$ & a. $6 E-02$ \\
\hline 120 & 174 & 167 & 164 & 160 & 155 & 151 & 147 & $: 43$ & $14 ?$ & 157 & is & 132 & (iv) & 122 & 121 \\
\hline 36.7 & $\pm d .3$ & 37.5 & 38.0 & 39.0 & 40.3 & +1.8 & 43.5 & 45.3 & 47,4 & 45.6 & $51 . ?$ & 54.3 & 5.3 & 59.3 & 52.3 \\
\hline$\varepsilon^{-}$ & 5 & $c^{-2}$ & PE-01 & $|E-j|$ & bE- 1 & $E E-91$ & $2 . \pi E-11$ & $2.1 \varepsilon-\$ 1$ & $7 E-n t$ & $(.7 E-1) 1$ & $5:-13 !$ & 1. $. Z E-31$ & $1,|E-0|$ & $9,4 E-32$ & $3,1)-12$ \\
\hline 19 & 175 & 170 & 165 & 161 & 157 & $1 \leq t$ & 149 & $1+6$ & 143 & $i \neq 0$ & 137 & $1: 4$ & $\mathrm{N2}$ & 130 & 120 \\
\hline$\$ 0,0$ & 40.1 & 911.6 & 41.2 & 42.2 & $\{3.3$ & 4.7 & 46.3 & 48.1 & 50.0 & 82.1 & 54.3 & 5.6 & 57.4 & b1.5 & 34.13 \\
\hline $0 E-01$ & $P E=0 t$ & 3. $2 \mathrm{E}-\mathrm{i} \mathrm{I}$ & 2.6E-j] & $5 \varepsilon-01$ & $4 \varepsilon-01$ & $2.3 \dot{z}=01$ & $2.25-01$ & $2.0 \mathrm{E}-01$ & SE-W1 & $1.5 E \cdot 01$ & $4 E-01$ & $2 E-31$ & :E-vì & $3.2 \mathrm{E}-102,2$ & $7.95-i 2$ \\
\hline 190 & 175 & $17 t$ & 167 & 162 & 158 & 155 & $15 t$ & 248 & 145 & $: 42$ & 199 & 157 & 135 & $1: 2$ & $: \Delta$ \\
\hline 43.3 & 43.5 & 43.9 & 44.5 & 45.3 & 46.4 & 47.7 & 49.2 & 50.9 & 52.7 & 54.7 & 5.8 & 59.0 & 61.5 & 53.7 & ti.2 \\
\hline & 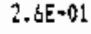 & $7 E-0 !$ & $z-0$ & - & $E=-0$ & $2 E-91$ & $N E-01$ & $9 E-5 !$ & $n E-\Delta]$ & !.5E-0! & . $4 t^{2}-11$ & $1.2 \xi-01$ & 1.JE-1): & $9,9 E-3: 2$ & D. $7 \mathrm{E}^{-172}$ \\
\hline $1 B$ & ir & $: 71$ & 167 & !b4 & 160 & : & 153 & $! 50$ & 167 & 144 & 141 & 199 & 137 & 1.5 & בנ: \\
\hline 46.7 & 45.3 & $67 .:$ & 47.3 & 4.5 & 49.6 & 50.3 & 52.2 & 57.7 & 55.5 & 57.3 & 57.3 & 61.5 & 53.7 & $66 . \dot{x}$ & 68.7 \\
\hline JE-01 & $5=-0 !$ & 2.SE-01 & 2. $₫-0 !$ & 2. IE-2)! & $2.1 E=01$ & $2.0 E-21$ & $1.9 E-0 !$ & EE-01 & SE-0! & AE-Dit & $3 E-91$ & $E-01$ & $9 E-42$ & $b \bar{\varepsilon}-12$ & $.4 E-02$ \\
\hline 180 & 178 & 172 & 168 & $! 65$ & 161 & 158 & 154 & !5! & 149 & :46 & $! 13$ & $1+1$ & 139 & 106 & :.35 \\
\hline 50.0 & 50.1 & 50.7 & 51.0 & 51.7 & 52.7 & 53.9 & 5.2 & 56.7 & $58 . j$ & 50.1 & 52,0 & 84.0 & 66.2 & be.t & 71..: \\
\hline
\end{tabular}

External Dose (rem) from Ground Contamination Estimated for $0000 z$ May 7 , 1986, for the Southeast Quadrant. Bearings are in Degrees and Distances are in Miles from Chernobyl. 


\begin{tabular}{|c|c|c|c|c|c|c|c|c|c|c|c|c|c|}
\hline & & & & & 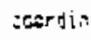 & of of & $g r: d$ & & & & & & \\
\hline 1 & : & $y$ & 4 & 5 & s & 1 & 8 & 9 & 20 & 11 & 12 & 14 & lt \\
\hline & 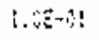 & GE. & & & & & & & ? I) & $\because-\because$ & $c=-12:$ & 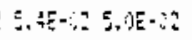 & $E+12$ \\
\hline 215 & تts & 119 & $\because n$ & $\because$ & $\therefore 5$ & 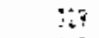 & $\because i$ & $3+4$ & $\because 2$ & 71 & $\because 3$ & b & 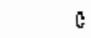 \\
\hline$\because ?$ & 3.4 & מ. & is & $\therefore$ & ei,: & 2 & 5. 7 & 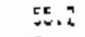 & $5 \times 7$ & 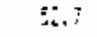 & $5 i, 7$ & $3 x .1$ & 50.15 \\
\hline$\varepsilon, 2 E-v_{2}$ & $0.7 E-12$ & $|.25-\mathrm{i}|$ & 2. 4 +E-31 & $2.95-51$ & 1.3ิE- & $1 . \mid\{-j \mid$ & $i . \mid E-81$ & $\therefore . . \pm+1 !$ & {$[.35-0 \mid$} & $1 . d E-11$ & 1.IE-@! $3 . \exists E-12$ & $0.0 E-025.56-62$ & $5.5 E-n 2$ \\
\hline$\because 3$ & it5 & $\therefore 7$ & 319 & in: & 324 & 227 & 300 & 30 & 30 & $j \neq 1$ & 34 & ZES & a \\
\hline 5.4 & s.: & 5..7 & ai. $\bar{E}$ & $59 . \dot{3}$ & 5.3 & 5 & 5.7 & $52 .:$ & 5.3 & ذ. & 47.7 & 46.9 & th. 7 \\
\hline & 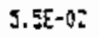 & $E-t$ & 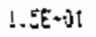 & $E-91$ & $E-0 !$ & $1,7 E-01$ & {$[.2 \varepsilon-01$} & $\mid, 2 E-1) !$ & $\mathrm{je}-0 !$ & 1. $5 E-13 t$ & 1. $+(-n-1,25-1)$ & 3. TE- -02 6. 3 .3E-J? & $E=5 ?$ \\
\hline 310 & $3 \hat{2}$ & 315 & $\$ 17$ & 315 & 522 & 325 & 395 & $3 !$ & 355 & 33 & 247 & 355 & c \\
\hline 30.2 & 5.7 & $81 . ?$ & 59.0 & fa.a & 54.7 & 52.7 & 50.9 & 49.2 & 47.7 & 18.4 & $\$ 4.5$ & 43.5 & $43, z$ \\
\hline$(\varepsilon-4)$ & $5+5 E-\sqrt{2}$ & $t E=0 ?$ & A. $\approx E-02^{\circ}$ & $1,35-01$ & 3. :E-D! & a.9E-Ll & $1.7 \mathrm{E}-0\}$ & ¿..E-u! & {$[.35-31$} & 1.SE-v1 & {$[. s[-0+1 \mid 1.5 E-0]$} & $1.1 E-018.5 E-12$ & $\therefore .0 E-v_{2}^{2}$ \\
\hline 398 & 310 & 312 & 315 & 317 & 320 & 323 & $52 b$ & sit & 33 & 357 & 345 & \$55 & 1) \\
\hline 54.4 & 61.5 & 59.4 & $\$ 6.6$ & 54.3 & 52.1 & 50.0 & 48.1 & 46.5 & 4.7 & $43 . j$ & 41.2 & 40.1 & 40.0 \\
\hline$g E-92$ & $5.9 E-192$ & $4.25-02$ & $8.75-02$ & $9.6 E-02$ & $2.15-01$ & $3.2 E-0 !$ & $2.7 E-01$ & $1.68-61$ & $1,3 E-0 \mid$ & $\{.5 \varepsilon-01$ & 1. TE-01 [..?E-01 & $1.5 E-01: . .: E-1]$ & $8.0 E-93$ \\
\hline 305 & 309 & 310 & 312 & 315 & $3: 7$ & 30 & 323 & 327 & 江 & 3 & 344 & 34 & 0 \\
\hline 62.5 & 53.5 & 56.3 & 54.2 & $5: .7$ & $\$ 9.6$ & $\$ 7.4$ & 45.3 & 45.5 & $4 ! .8$ & 90,3 & 39.0 & 54.8 & 34.2 \\
\hline$+5-12$ & $6.35-02$ & 6. $4 E-32$ & $1 E-22$ & $J E-32$ & $\{.2 E+0 !$ & $\because E-D$ & $3.2 E-M 1$ & 3. $2(2-9)\}$ & SE-01 & $55-01$ & $7 E+01 \quad 1.5 E-81$ & 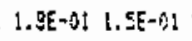 & $9 . B E-02$ \\
\hline 305 & 50 & 307 & 167 & $\Sigma ! \hat{s}$ & $\because 5$ & 113 & 321 & 325 & 37 & ji3 & גio & $\mathbb{N}_{4}$ & $\hat{i}$ \\
\hline 50.: & 53. & 54.7 & 52.1 & $\$ 7.6$ & 17.: & 44.9 & $42 .+7$ & 40.7 & IE. 7 & 8.3 & E.? & 33.5 & 3.3 \\
\hline$?\{-02$ & $1.2 \div-02$ & $1, j \bar{c}-2 \hat{2}$ & $7.7 E \cdot \mathrm{i} ?$ & $8.15-22$ & a. $9 E-02$ & {$[.4 E \cdot 0]$} & 2. (E- E-1) & $F E-1 ! !$ & 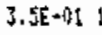 & $J E-1) !$ & $7 \varepsilon-0 !$ & $2.25-01 \quad 2.0 \varepsilon-01$ & $1 . j E-01$ \\
\hline 30 & 302 & 304 & 396 & 369 & 311 & 315 & $3 ! 9$ & 32 & $i 25$ & 30 & 341 & 353 & 0 \\
\hline 59.3 & 55.5 & $3 . .7$ & 59.7 & 4.8 & 44.3 & 42.4 & $40 . !$ & 59.0 & 话.! & 34.3 & $=2.8$ & U.2.2 & 3,0 \\
\hline$E=02$ & $E-122$ & -02 & 2 & $E-102$ & $9.2 E-82$ & $\mid-1)$ & $\mathrm{E}=\mathrm{Bl}$ & $5-01$ & $! E-\$ ; \mid$ & 4. $1 \hat{E}-01$ & $9 E-1) \quad 2,65-0:$ & $2.45-013.55-01$ & $75-4 !$ \\
\hline 288 & 299 & 301 & 503 & 306 & 309 & 311 & 315 & 319 & 323 & 327 & 359 & 352 & 0 \\
\hline 56.7 & 5.7 & 50.9 & 48.1 & 45.3 & 42.7 & 90.1 & 37.7 & 35.4 & 33.3 & 31.4 & 29.5 & 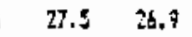 & $2 b .7$ \\
\hline$[-0 \mid$ & $1.2 E-0,1$ & $9.3 E-02$ & $9.3 E-02$ & $9.5 E-12$ & $9.8 E-12$ & :.0E-0! & I. $.5-0 !$ & $1.9 E-01$ & 3.JE-91 : & $3.7 E-191$ & 4. $d E-31$ 2. $[E-0\}$ & $2.7 E-013.3 E-01$ & $6 E-01$ \\
\hline 295 & 296 & 238 & 300 & 302 & 304 & $30 ?$ & 311 & 315 & 319 & 324 & iss & $35 !$ & 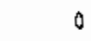 \\
\hline 5.2 & 52.2 & 49.2 & 46.5 & 43.5 & 40.7 & 78.0 & 35.4 & 5.0 & 30.7 & 28.7 & 25.4 & 23.6 & $2 \pi .5$ \\
\hline$P E-O I$ & $2.65-01$ & $1.9 E-31$ & 1. $9 E-01$ & $1.1 E-0 !$ & $! . ! E-0 !$ & $E=0 !$ & $1.1 E-21$ & 1. $3 E-91$ & $2.3 \mathrm{t}-01.3$ & $3.6 E-0 !$ & $4,8 E-014,1 E-01$ & $3.8 E-013.9 E-91$ & $3.7 E-01$ \\
\hline 291 & 293 & 294 & 396 & 298 & 300 & 303 & $30 \mathrm{O}$ & 310 & 315 & 320 & 236 & 350 & 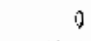 \\
\hline 53.9 & 50.8 & $\$ 7.7$ & 44.7 & $\$ 1.9$ & 38.9 & $i b .1$ & 33.2 & 30.7 & 泹. 3 & 26.0 & 24,0 & 30.2 & 30.6 \\
\hline |E-?! & $|E-i| d$ & $E-41$ & $-1) !$ & $E-01$ & $E+1)$ & $\{-1$ & $E-1$ & 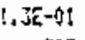 & $E=0 ! 2$ & PE-OI! & $t E-4 !$ & JE-91 & SE-91 \\
\hline 259 & ?B9 & 291 & 37 & 294 & 295 & 209 & $30 !$ & 725 & 309 & 315 & ?:? & 349 & b) \\
\hline 52.7 & 49.5 & $\$ 8.4$ & 43.3 & 40.3 & 37.5 & 84.5 & 31.4 & 28.7 & 26.0 & 23.6 & 21.3 & 17.2 & 18.7 \\
\hline$\xi \dot{E}+01$ & 2.JE-01 & $3.02-01$ & $3.6 \mathrm{E}=\mathbf{1} t$ & 3. $7 E-B b$ & 3.?E-6! & $3.1 E-0 !$ & $1.95-91$ & i.EE-OL & 1. $7[-91:$ & $2.0 E+0 i$ & Z.3E-i1 (6.JE-0)! & 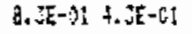 & 5. $د \varepsilon-4 !$ \\
\hline $20:$ & 205 & 297 & 238 & 289 & 291 & 293 & 296 & 299 & 383 & 298 & IIS & 545 & ? \\
\hline $5: .7$ & 48.5 & 45.3 & 46.2 & 89.0 & 35.9 & 32.8 & 29.8 & 26.9 & 24.0 & 24.3 & $: 9.9$ & 12.7 & 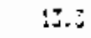 \\
\hline EE-OI & $2 E-9 t$ & $z-01$ & $2 E-4$ & $7 E-01$ & $3 . j(2-01$ & $9 E-1 \mid 1$ & HE-D! & $E[-0 t$ & $2 . \Delta E-0$ & $\neq \varepsilon-0 !$ & $F E-0 ! b .2 E-3 !$ & $9.9 \varepsilon=0 ! 7.5 E-31$ & $4 E-90$ \\
\hline $25 !$ & 282 & 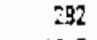 & 294 & 295 & $3 B E$ & 283 & 290 & 293 & 296 & 500 & 206 & $3+1$ & 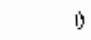 \\
\hline 51.3 & 97.7 & 44.5 & ti. ${ }^{*}$ & 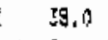 & 24.8 & $2 ! .3$ & 23.5 & 25.4 & 23.4 & 19.4 & 14.1 & 214.5 & 10.3 \\
\hline E-SIt & E-0! & $E-0 !$ & SE-D! & $1.6 E=01$ & $E-01$ & $35-01$ & $7.65-4 !$ & $4.0 E-01$ & S. $\{E-3)$ & $6.5 E-4 !$ & $4.35-11 \quad 5,2 E-01$ & $1,2 E+\infty 0 \quad 1,3 E+40$ & $.2 E+50$ \\
\hline 277 & 278 & 278 & 2 & 280 & 25.1 & רE: & 284 & 285 & 298 & $39 t$ & 296 & 850 & 0 \\
\hline 50.4 & $\$ 3.1$ & 43.3 & 40.5 & 37.3 & 39.0 & 30.7 & 27.5 & 24.3 & 24.1 & 19.0 & 14.9 & i.5 & b. 3 \\
\hline$(E-1) !$ & $1.1 E-0 !$ & $.2 E-3]$ & $E-01$ & $E-0 I$ & !.5E-0! & $z-6 !$ & $\therefore ! E-0 !$ & 2. YE-OL & [E-31 & ธ..ZE- 11 & 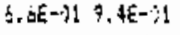 & $\varepsilon+10 ?$ & $i . \$ E+00$ \\
\hline 273 & 274 & 274 & 274 & 275 & 275 & $27 \mathrm{~b}$ & 277 & 279 & 277 & $26 i$ & 208 & $3 \mathfrak{3}$ & 0 \\
\hline 50.1 & to.a & 43.5 & 40.1 & 24.3 & 33.5 & 30.2 & 26.9 & 23.6 & 20.3 & 17.0 & 13.7 & 4.7 & 3.3 \\
\hline $5-1$ & $E=0$ & $E=$ & $E-D 1$ & $E-0$ & 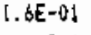 & $E=0$ & & & $F_{-}$ & GE- & $3 E-016.9 E-$ & $9.2 \Sigma-01 \quad 1.2 E+00$ & $E+60$ \\
\hline i: & 270 & 2) & 7 & 270 & 270 & 270 & 270 & 271 & $2 \pi$ & 270 & 270 & 37 & 0 \\
\hline 50.0 & 46.7 & 43.3 & 40.3 & 36.7 & 35.3 & 30,0 & 26.7 & 23.5 & 23.0 & 16.7 & 13.3 & 5.7 & 0.8 \\
\hline
\end{tabular}

Whole Body Dose (rem) from Inhalation Estimated for 00002 May 7, 1986, for the Northwest Quadrant. Bearings are in Degrees and Distances are in Miles from Chernoby?. 


\begin{tabular}{|c|c|c|c|c|c|c|c|c|c|c|c|c|c|c|c|}
\hline & & & & & coser & 0 & the $y^{2}$ & at: & & & & & & & \\
\hline 1 & 2 & 3 & 1 & $s$ & b & 7 & 8 & 9 & 10 & 11 & 12 & 13 & 14 & 18 & 15 \\
\hline$: E-3$ & $E-01$ & $3 E-0$ & $6 \varepsilon-01$ & 4. $35-1) ! 5$ & S. 4E. & $4.82+0$ & J.bE- & te- & $j E-01$ & 6. $\tilde{6} E-1$ & $9.6 E^{-1}$ & $1.3 E+000$ & 9. $\Delta E-Q !$ & $1 . z E+0$ & $O E=00$ \\
\hline 256 & 265 & .55 & $2 t 5$ & 264 & 264 & 25 & 262 & $26 t$ & 260 & 258 & 255 & 251 & 243 & 225 & 180 \\
\hline 5 & 46.8 & 43.5 & th. $i$ & 24.9 & 3.5 & 30.2 & Za. & $\because+.6$ & 79.2 & 17.0 & 0.7 & 10.5 & 7.5 & 4.7 & 3.3 \\
\hline $2 E-01$ & $1.3 E-01$ & $2+|\varepsilon-\hat{v}|$ & $5.3 E=01$ & 6. $2 \varepsilon-01$ & $6.6 E-01$ & $4.15-106$ & $4, A E-81$ & $4.8 E-0:$ & $6 E-01$ & $6.3 E-31$. & 4. E-0! & $8.1 E=01$ & DE $+00 ?$ & $E+0$ & $O E+00$ \\
\hline 262 & $26 !$ & $26 !$ & 260 & 259 & 258 & 257 & 298 & 254 & $25 !$ & 248 & 243 & 238 & 225 & 206 & 180 \\
\hline 50.4 & 47,1 & 43.8 & 40.6 & 37.3 & 34.0 & 30.7 & 77.5 & $24 . j$ & 24.1 & 18.0 & 14.9 & 12.0 & 9.4 & 7.5 & 6.7 \\
\hline 1.5E-11: & 2. $1 E-10 t$ & 4. $9 F-01$ & $7.7 t-12 t$ & $+\varepsilon-01:=$ & 5.7E-01 & $3.4 E-3) t$ & $3.3 E+01$ & $3.5 E-01$ & $3.7 E-0 !$ & $4.7 \varepsilon-01$ & $6.0 E-01 ?$ & 7. $\delta E-0 ! !$ & $1.5 E+00$ & AE+BO & $2 E+1 !$ \\
\hline 258 & 257 & 257 & 255 & 254 & $2 \pi$ & 251 & 249 & 246 & 243 & 239 & 253 & $2 \pi 5$ & 213 & 198 & 180 \\
\hline $5 ! .0$ & 47.7 & 44.9 & $\$ 1.2$ & 39.0 & 34.8 & 31.6 & 28.5 & 25.4 & $2 \pi .7$ & 19.8 & 16.7 & 14.1 & 12.0 & 10.5 & 19.0 \\
\hline $9 E-01$ & 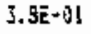 & 4, ate- l] & $3.8 E-0 !$ & 2. $\Delta E=012$ & $2.2 E-01$ & $2.2 t-61$ & $2,4 E-01$ & $2.7 E-01$ & 3.JE -01 & 4.1E-0I & 5. $2 F-01$ & B. $35-01$ & $1.4 E+0.0$ & $1.15+00$ & $E E+\infty 0$ \\
\hline $2 \leqslant 5$ & 254 & 252 & 28 & 250 & 248 & 246 & 243 & 240 & 278 & 231 & 225 & 216 & 206 & 194 & 180 \\
\hline 5t.7 & 48.5 & 45.3 & 42.2 & 39.0 & 53.9 & 32.8 & 29.8 & 26.7 & 24.0 & 21.3 & 19.9 & 16.7 & 14.9 & 13.7 & 15.3 \\
\hline & 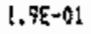 & -6 & $\nabla r$ & $5-012:$ & 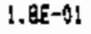 & $=-61$ & $z-n$ & 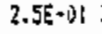 & $\theta=0 t$ & $k^{-01}$ & $0 t-01\}$ & $9.8 \mathrm{t}-0 \mathrm{t}]$ & $1.0 E+00$ & $0 E+00$ & {$[E-01$} \\
\hline 251 & 250 & 240 & 247 & 245 & 243 & 240 & 237 & 234 & 230 & 227 & 218 & 210 & 201 & 191 & 150 \\
\hline 52.7 & 49.6 & 46.4 & 43.3 & 40.3 & 57.3 & 34.3 & I.. & 28.7 & 26.0 & 23.6 & 24.3 & 19.4 & 19.0 & 17.0 & 16.7 \\
\hline 0 & $E-0$ & $E-01$ & 1.SE-g1 & I. $3 \mathrm{E}-01$ & 1.JE-01 & $1.8[-81$ & $O E=0 I$ & IE-0! & $2.9 E-\Delta !$ & $z E-01$ & $55-61$ & $\{\varepsilon-0 !$ & $25-018$ & $2 \varepsilon-01$ & eE-St \\
\hline 218 & 246 & 245 & 243 & 241 & 259 & 236 & 203 & 229 & 223 & 219 & 213 & 206 & 198 & 199 & 180 \\
\hline 53.9 & 50.9 & 17,7 & 44.7 & 11.8 & SB. 9 & 36.1 & 33.3 & 30.7 & 28.3 & 26.0 & 24.0. & 22.4 & 21.1 & 20.3 & 20.0 \\
\hline $3 E-01$ & EE-61 & $1.1 E-01$ & $4 E-01$ & $E-011$ & $6-01$ & $E=-01$ & $E-01$ & $\varepsilon=01$ & 2. $7 E-01$ & $4.2 E-01$ & EE-at & $3 E-31$ & $6.0 E-01$ & b.5E-01 & 6.EE-Ct \\
\hline 244 & 243 & 241 & 234 & 237 & 235 & 752 & 228 & 229 & 220 & 215 & 209 & 205 & 195 & 189 & 180 \\
\hline 55.2 & 4 & 49.2 & 3 & 45.5 & 40.7 & 30.0 & 53.4 & 33.0 & 30.7 & 28.7 & 26.9 & 25.4 & 24.3 & 23.8 & J.2. \\
\hline $25-0 !$ & $\varepsilon-0\}$ & $E-01$ & E. & $5 E-0 ! !$ & $b E-0 !$ & $8 E-01$ & 1 & $E-0 !$ & $B E-91$ & 1.6E-01 3 & $3 E-01$ & !E- $01:$ & EE-0!: & 5. $t E-0 !:$ & $6 E-01$ \\
\hline 241 & 240 & 258 & 256 & $2 \pi$ & 221 & 228 & 225 & 221 & 216 & 212 & 206 & 200 & 194 & 187 & 180 \\
\hline 56.7 & 35.7 & 50.7 & 48.1 & 45.3 & 42.7 & 40.1 & 37.7 & 35.4 & 33.3 & 31.4 & $x, 8$ & 28.5 & 27.5 & 26.9 & $2 s .7$ \\
\hline & 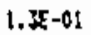 & E- $=9 !$ & 1 & $\delta E=0 !]$ & $=-a l$ & 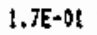 & $E=9 !$ & -01 & $\varepsilon-01$ & {$[-013$} & $E-01\}$ & $E-01$ & $x-01$ & EE-01 & $7 E-21$ \\
\hline 23 & 257 & 23 & 3 & 230 & 288 & 25 & $22 !$ & 317 & 213 & 209 & 203 & 198 & 192 & 10 & 180 \\
\hline 58.3 & 35.5 & 52.7 & 50.0 & 47.4 & 44.8 & 2.4 & \$0.1 & 58.0 & 36.1 & 34.3 & 58.3 & 31.6 & 30.7 & 30.2 & 30.0 \\
\hline $2[-01$ & $3 E-01$ & $1.3 E-01$ & 1.4E-01 & $E=011$ & $1.6 E-01$ & $E-01$ & $E=01$ & |E-3! & $2.5 E-01$ & 2.SE-01 2 & $2.75-013$ & $3.55-01$ & $1.6 \mathrm{E}-\mathrm{-1} ?$ & $9 E-91$ & $\pi=01$ \\
\hline $23 b$ & T) & $25 \%$ & & 227 & 225 & 221 & 218 & 214 & $2: 0$ & 206 & 201 & 196 & 19: & IEs & 190 \\
\hline 50.1 & 07.3 & 54.7 & 52.1 & 49.5 & 47.1 & 4.9 & 42.7 & 40.7 & 38.9 & 37.3 & 35.9 & 34.8 & 24.0 & 33.5 & $\therefore, 2$ \\
\hline (c) & $0+01$ & $1.3 \mathrm{E}-01$ & $4 \varepsilon-01$ & EE-0) 1 & $6 E-\$ 1$ & $\mathrm{t}=0 !$ & $3 E-01$ & $\mid \Sigma-0 !$ & 2.JE=01 & $2.5 E-01 ?$ & $2.7 E-0: 3$ & $3.2 \mathrm{E}-0 !$ & 4.0E-01 3 & IE-S1: & $3 E-0 l$ \\
\hline 37 & 231 & 229 & 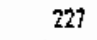 & 225 & 2 & 19 & 216 & 212 & 208 & 204 & 199 & 195 & 190 & 185 & 180 \\
\hline 62.9 & 99.2 & 56.8 & & 51.7 & 49.6 & 47.4 & 45.2 & 43.5 & 41.9 & $40 . \overrightarrow{3}$ & 39.0 & 39.0 & 57.3 & 36.9 & 36.7 \\
\hline & $z^{-1}$ & $=-0$ & 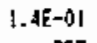 & & 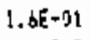 & 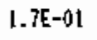 & 1 & 2 & 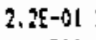 & {$[E-0 \mid 2$} & E-01 & {$[E-0 \mid 5$} & $E=0$ & $\varepsilon-01$ & $\varepsilon-01$ \\
\hline 23 & 22 & 22 & 22 & 222 & 7 & $21 d$ & & 10 & 206 & 202 & 178 & 194 & 189 & 184 & 180 \\
\hline 54.0 & b1.5 & 59.0 & 56.6 & 54.3 & $52 .:$ & 50.0 & 49.1 & 46.3 & 44.7 & 43.3 & 42.2 & 41.2 & 40.5 & 10.1 & 40.0 \\
\hline$(E-1) t$ & $3 E-01$ & !.JE-01 & $1,+2 E-01$ & 1.5E-01 & $1.6 E-01$ & $E-01$ & $1.9 E-91\}$ & $E-01$ & IE -01 & {$[E-c 12$} & $\mid E-A 1]$ & $E+013$ & $\{-0 \mid$ & $E-0 ! 3$ & 3. ZF-01 \\
\hline 229 & 227 & 225 & & 220 & 217 & 214 & 211 & 68 & 204 & 201 & 197 & 192 & 189 & เ64 & 180 \\
\hline 66.2 & 63.7 & b1.j & 59.0 & 56.8 & 54.7 & 52.7 & 50.9 & 49.2 & 47.7 & 4.4 & 45.3 & 4.5 & 43.8 & 43.5 & 43.3 \\
\hline $2 E-01$ & $2 E-01$ & 3E-91 & $4 \varepsilon-0$ & $5 E-01 !$ & bE-0 & TE-01 & PE. & $\mathrm{BE}-\mathrm{0}$ & $1 E-01$ & $2.2 E-01$ & $3 E-912$ & $E-0 ! 2$ & $2.8 \mathrm{E}-0 \mathrm{t} 2$ & $2.7 E-\hat{1}]$ & J. $\hat{v} E-i_{i}[$ \\
\hline .2 & 12 & 22 & & 79 & 5 & 212 & & 206 & 203 & 179 & 195 & 192 & 1BE & 194 & 120 \\
\hline 68. & & 63.7 & & 59.3 & 57.3 & 59.5 & 53.7 & 52.2 & 50.8 & 19.6 & 48.5 & 47.7 & $\$ 7.1$ & 46.8 & 46.7 \\
\hline & s & & & $5 E-0 t$ & 1. $b E=01$ & $1.7 E-01$ & t. $8 E-0 !$ & $\mathrm{DE}-0 !$ & 2. UE-01 & 2. $\triangle E-012$ & 2. $2 E-0 ! 2$ & 2. $.6 E-01 ?$ & 2.5E-01? & 2.5E-G1 ? & $2.75-1) 1$ \\
\hline 225 & 20 & 370 & 21 & 316 & 213 & 210 & 208 & 05 & 201 & 198 & 194 & 191 & 197 & 183 & $1 B 0$ \\
\hline$n .7$ & 68.4 & ba. 2 & 64.0 & 62.0 & 60.1 & 58.2 & 56.7 & 39.2 & 53.9 & 52.7 & 51.7 & 51.0 & 50.4 & 50.1 & 50.0 \\
\hline
\end{tabular}

Whole Body Dose (rem) from Inhalation Estimated for 00002 May 7, 1986. for the Southwest Quadrant. Bearings are in Degrees and Distances are in Miles from Chernobyl. 


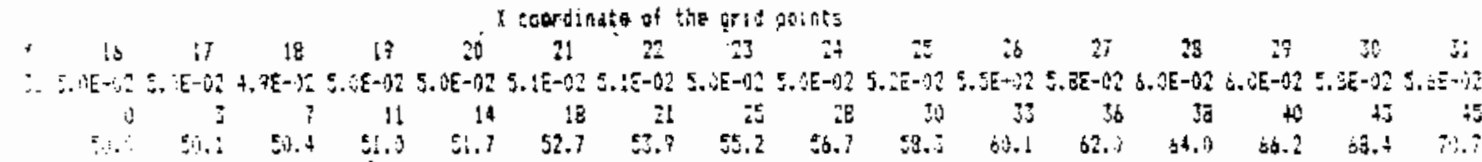

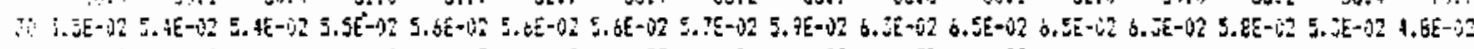

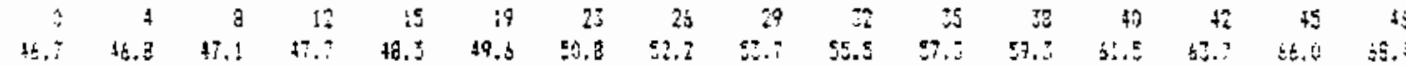

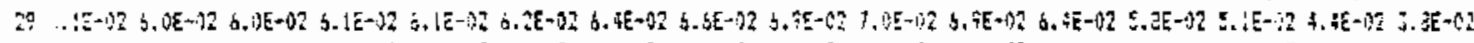

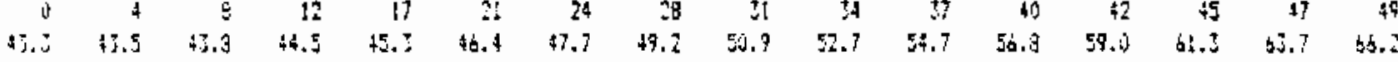

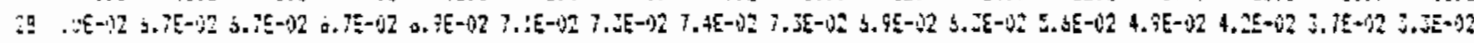

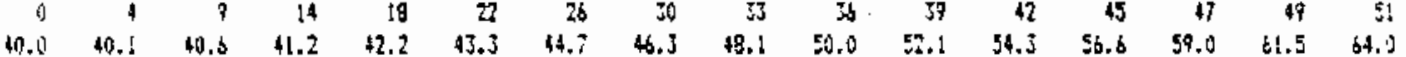

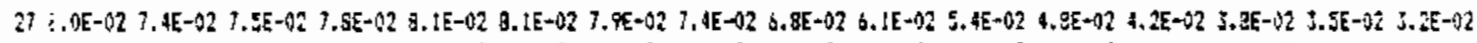

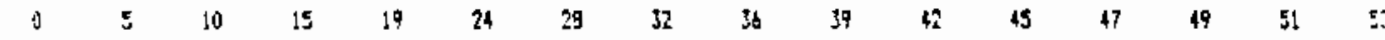

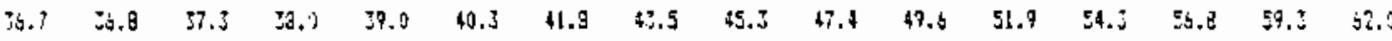

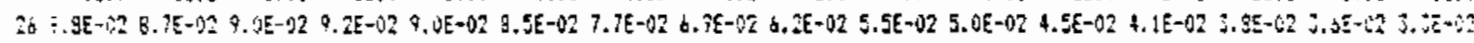

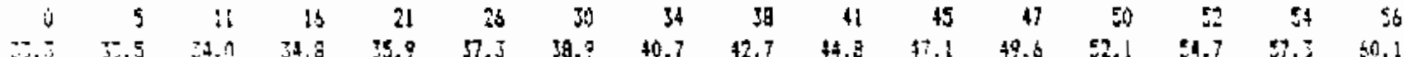

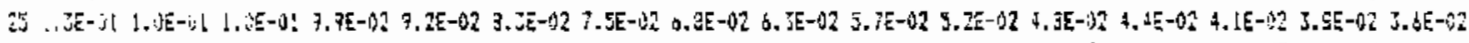
$\begin{array}{llllllllllllllll}0 & 3 & 12 & 19 & 23 & 29 & 3 & 37 & 41 & 45 & 48 & 50 & 53 & 55 & 57 & 57\end{array}$

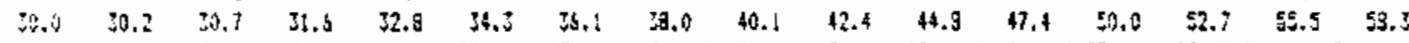

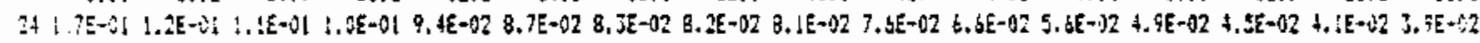

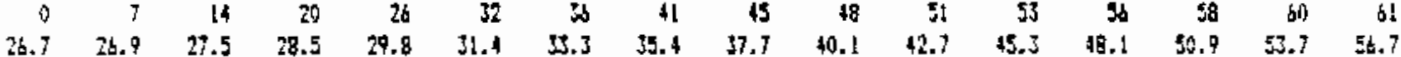

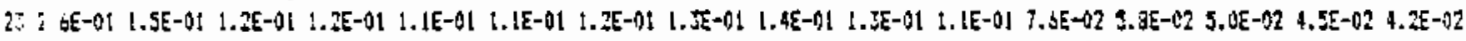

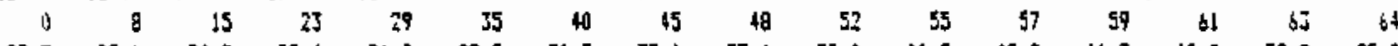
$\begin{array}{llllllllllllllll}23.3 & 30.5 & 24.3 & 25.4 & 26.9 & 28.7 & 30.7 & 33.0 & 35.4 & 38.0 & 40.7 & 43.5 & 16.3 & 49.2 & 52.2 & 55.2\end{array}$

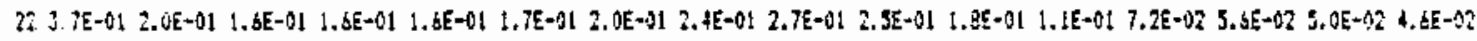

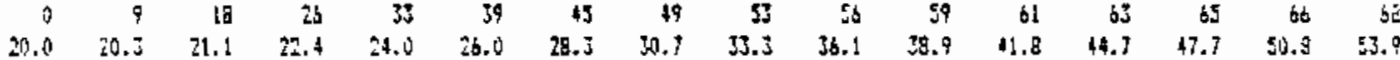

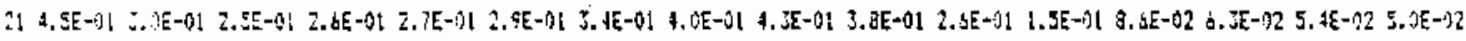

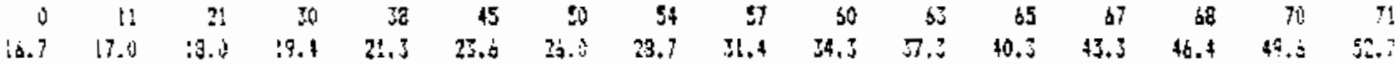

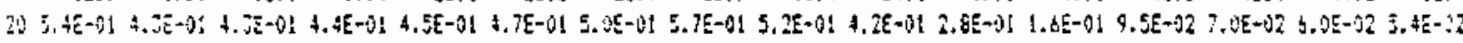

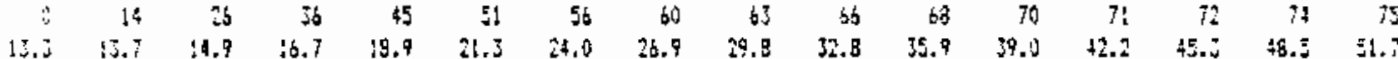

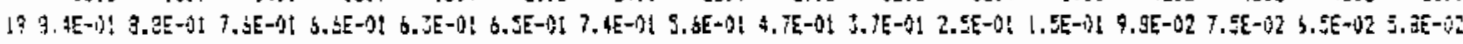

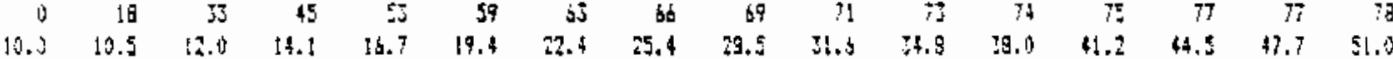

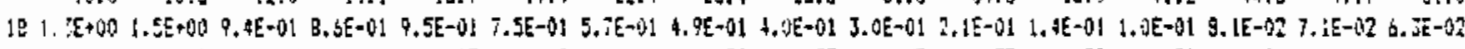

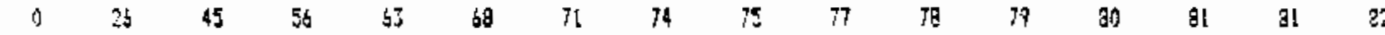

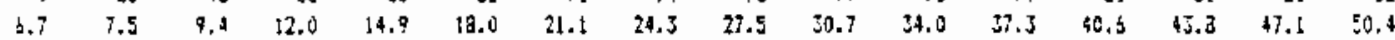

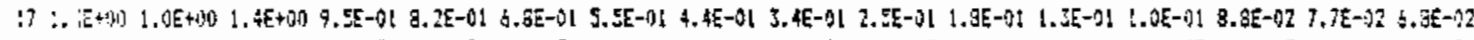

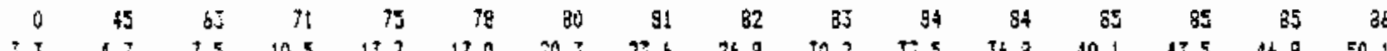

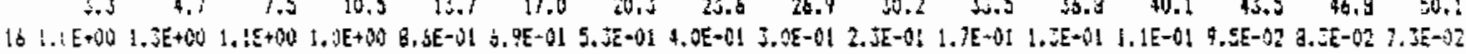

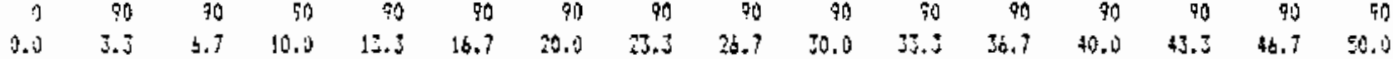

Whole Body Dose (rem) from Inhalation Estimated for $0000 z$ May 7, 1986, for the Northeast Quadrant. Bearings are in Degrees and Distances are in Miles from Chernobyl. 


\begin{tabular}{|c|c|c|c|c|c|c|c|c|c|c|c|c|c|c|c|}
\hline & & & & & & & & & & & & & & & \\
\hline ; & 17 & 12 & 19 & 20 & 31 & 23 & $\because 2$ & 24 & 25 & is & 27 & $: 3$ & 29 & 30 & $\because$ \\
\hline WE: & $\therefore E+i) 0$ & Erenit & 4 & $E-$ & $=$ & $E-\eta !$ & $E-4 t$ & is-1); & 2.ZE-fit & $T E-0:$ & $1, \pm E-1) !$ & 1. IE- 0 ! & $1.2 E-1,1$ & $.2 E-32$ & $i E-i ; z$ \\
\hline $16: 3$ & $1: 5$ & 116 & 108 & 104 & $10 !$ & $8:$ & 98 & 73 & 98 & 唡 & 75 & 94 & it & $9+$ & 73 \\
\hline 3.2 & 4.7 & 7.5 & 20.5 & [2.? & $\therefore !$ & $\therefore i$ & $\therefore, b$ & 3.9 & 2 & S. & S. & 0.1 & +3.5 & 46.5 & $5 \mathrm{in}:$ \\
\hline$(8 E+01)$ & $2.4 E+00$ & $1,45+00$ & $1.1 E+10$ & $7.3 E-11$ & L. $2 E-\hat{u}:$ & 4. $7 \pm-15 !$ & $\xi=-11$ & :.PE-:!! & A.IE-: :! & 1. 乘-6! & $\{. E E-3 \mid$ & 1. & $1.15+v i$ & 8.bE- - $22=$ & $\{E-132$ \\
\hline 180 & 153 & 1.5 & 125 & lib & $11 !$ & 108 & 105 & $: 24$ & $: 22$ & $: 21$ & 649 & $\$ 9$ & 98 & 79 & 97 \\
\hline 6.7 & 7.5 & 9.5 & 12.0 & 14.7 & 18.0 & $2 . .1$ & 24.5 & $2 . .8$ & 活: & 35.0 & 3.3 & 40.6 & 43.3 & $\$ 7 . \$$ & 5.4 \\
\hline+0. & AE+ & - & $E-0$ & 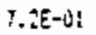 & 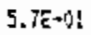 & $E-A !$ & $\varepsilon E-9$ & 2.JE-D! & $z-31$ & $1 . \sqrt{E}-1$ & $1, \frac{(4-1)}{(2)} 1$ & $\{E=01$ & $1.2 E-1$ & JE-OL & $35-32$ \\
\hline$! 50$ & 161 & $1+6$ & 175 & 125 & 120 & $1: 5$ & 113 & 190 & 100 & : & 105 & 104 & $\log 2$ & $: 02$ & (D) \\
\hline ?ח & li). 5 & 12.0 & $\because \div .1$ & is.? & 19.4 & 23.4 & $2 \approx .4$ & 39.5 & 2.6 & 24.3 & ‡a.. & $4: .2$ & $\$ 4.5$ & $47 .:$ & $\$ 1 . j$ \\
\hline $1 \subseteq+00$ & $25+00$ & $7.2 E-i, 1$ & 7.LE-D! & $6.45-\$ 1$ & 5. $2 E-0 !$ & A. $.3 E-01$ & $3.4 E-v 1$ & $2.8 \mathrm{~g}-0 \mathrm{l}$ & $2.3 E-04$ & $2 . \because E-A 1$ & $1.75-41$ & i. $4(E-1) t$ & 1. $25-4 !$ & L. $\{E-1)\} ?$ & $9.7 E-j 2$ \\
\hline 130 & 165 & 15 & 145 & 128 & 228 & 123 & 119 & 115 & 11: & : $: !$ & $: 09$ & 10 & 107 & $1: 5$ & 107 \\
\hline $\mathfrak{i j . j}$ & 13.7 & 14.9 & 16.7 & $: 8.8$ & 21.3 & 24.0 & $2 k .9$ & 29.8 & $\because 2.8$ & teq & $27.0^{2}$ & $4 \hat{2}=2$ & $45 . j$ & $4 \hat{A} . \bar{\Xi}$ & 51.7 \\
\hline $9 . ! 5-3 !$ & $1.0 E+\$ 0$ & $9 .|E-9|$ & 6.7E-01 & ง. $75-01$ & 4.9E-0! & 4. . $E-0 t$ & S.ZE-01 & 2. $8[-\rightarrow) !$ & $2.4 E-9 !$ & $2.3 E-0 !$ & $(.7 \varepsilon-1) 1$ & 1.SE=01 & 1. $\because E-81$ & 1, LE $-1: !$ & $9.9 E-12$ \\
\hline 180 & 168 & 19 & 149 & $14 t$ & its & 129 & 125 & $1 \hat{2}$ & $1: 9$ & 13 & 114 & 112 & 111 & 109 & 108 \\
\hline 16.7 & 17.3 & 19.0 & 19.4 & $24 . J$ & 3.6 & 26.0 & 3.7 & 21.7 & inj.j & 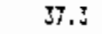 & $17+4$ & 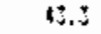 & 46.4 & 49.3 & 52.7 \\
\hline $3 E-01$ & $32-1)$ & $t=6 !$ & $2-0 !$ & $5-3 !$ & $A E-\dot{i} \mid$ & $E E-9 t$ & $E E-01$ & $B E-0 ! !$ & E-0! & $1 t-\theta i$ & $25-01$ & $6 E-01$ & $\overline{\mathrm{J}}-0 \mathrm{O}$ & $\hat{x E-\hat{\Delta L}}$ & DE- 1 \\
\hline 120 & 179 & 161 & 10 & 146 & 140 & 175 & 100 & 125 & $: 23$ & 20 & 18 & lí & 114 & $1: 2$ & :1: \\
\hline 20.0 & 20.3 & 21.1 & $2 \approx .4$ & 34.0 & 26.2 & 29.3 & 20.7 & 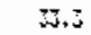 & $2 t .1$ & 8.8 & 41.8 & 44.7 & $\$+1$ & 50.9 & 5.9 \\
\hline & & 28 & $=\sqrt{2}$ & 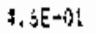 & $E=0 ?$ & $2-01$ & $15-01$ & $E-1$ & $4 \vec{t}-01$ & {$[E-n !$} & $3 E-0 !$ & tE(-1)! & $1, f E-g \mid$ & $2 E-31$ & LE- \\
\hline $18 i$ & 171 & $16 d$ & ط. & 15 & 144 & 1.7 & $1 \pi$ & 121 & $: 27$ & 124 & 122 & 20 & $1: 9$ & : & $: 15$ \\
\hline 20.3 & 23.6 & 24.3 & 25.4 & -26.7 & 29.7 & 约.7 & ) & 35.4 & 53.9 & 49.6 & 43.5 & 45.3 & 49.2 & 52.3 & 55.2 \\
\hline 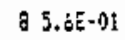 & t. $25-01$ & $5 F-i n$ & PE-OAt & A. $1 E-D !$ & $3.7 \mathrm{E}=0 \mathrm{l}$ & 3. AE-01 & CE-O: & 2.7E-OI & $+E=01$ & 2. $1 E-01$ & $1.9 \mathrm{E}-01$ & $1.6 \vec{E}-01$ & $1,+2 E-8:$ & $2 E-31$ & $\{.\{E-1\} !$ \\
\hline 180 & 172 & 165 & 159 & 153 & 147 & 143 & 139 & 135 & $i 31$ & 128 & t.2b & 123 & 121 & $1: 9$ & 118 \\
\hline 26.7 & 26.9 & 27.5 & 29.5 & 29.8 & 31.4 & 33.3 & 35.4 & 57.7 & 10.1 & 42. & 45.3 & 48.1 & 50.9 & $\$ 3.7$ & Sh. I \\
\hline $7 \varepsilon-0 !$ & 5.JE-0i & $7 t-0 t$ & $0 E-01$ & $1 E=0 !$ & $\varepsilon-9$ & $2 E-01$ & $Q E-01$ & SE-vt & $4 E-01$ & {$[E-0 !$} & FE-OI & $: .35-9 !$ & L. $4 E-D t$ & $1 . \pm E-\$ 1$ & $1.1 E-0 !$ \\
\hline 180 & 175 & 167 & $: 61$ & 156 & 100 & 146 & 142 & 8עו & te & 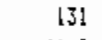 & 129 & 126 & 124 & 122 & 120 \\
\hline 30.0 & 30.2 & 20.7 & 31.5 & $72 . \mathrm{a}$ & 54.3 & 36.1 & $99.1)$ & 40.1 & 22.4 & 74.8 & 47,4 & 50.0 & 52.7 & $\$ 3.5$ & $53 .:$ \\
\hline $1 E-0$ & $S E-A$ & $2 E-31$ & $=-01$ & $E-0 !$ & $\mathrm{BE}+\mathrm{G!}$ & $\mid E-\$ 1$ & SE-01 & $b E-9 t$ & $\mathrm{EE}=01$ & $15-9 !$ & $9 €-01$ & dE-OI & $4 E=0 !$ & $3 E-\$ !$ & $1 E-01$ \\
\hline 180 & 174 & 19t & 153 & 153 & 153 & 149 & $: 45$ & 141 & 173 & 105 & 13 & 129 & $: 27$ & 179 & $12 z$ \\
\hline 3.5. & 33.5 & $\therefore 4.0$ & 54.3 & 25.9 & 37.5 & 38.9 & 40.7 & 42.7 & $+4,8$ & 47.1 & 99.6 & 52.1 & 5.7 & ST: & Siv.: \\
\hline$[E-3 !$ & 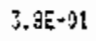 & $3 \varepsilon-91$ & $4 E-01$ & J.*t-\$1 & $t e-v t$ & $9 E-0 !$ & {$[2-3 !$} & 2.5E-0! & JE-t̂t & 2.BE-ग & $1.3 E=01$ & $\therefore . b E-01$ & 1. $4[-0]$ & $1.3 E-A 1$ & :. $\{[E-1\} !$ \\
\hline !at & 174 & 189 & 164 & 100 & 155 & 151 & 197 & 115 & t) & $! 7$ & 155 & $1 \pm 2$ & 120 & 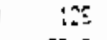 & $\therefore 6$ \\
\hline 36.7 & 36.3 & 37.3 & 39.10 & 54.7 & 40.3 & 41.3 & 43.5 & 15.2 & 47,4 & 49.6 & 54.7 & 54.7 & 56.8 & Si.: & 52.1) \\
\hline $4 E-51$ & J. $+E-01$ & $3.5 E-0 !$ & $1 E-0 t$ & Cf-DI & SE-3I & $3 E-41$ & bE- -31 & $4 E-\infty 1$ & $85-01$ & $\varepsilon E-0 !$ & $a E-0 !$ & $5 E-21$ & $f \vec{c}-8 \hat{b}$ & $\mathrm{JE}-\hat{\mathrm{y}} \mathrm{i}$ & !:-\$! \\
\hline 180 & 175 & (7) & 165 & 161 & 157 & 153 & 149 & 146 & 143 & 10 & 187 & lis & 122 & 20 & $1: 9$ \\
\hline 40.0 & 40.1 & 40.6 & 41.2 & 12.2 & $\$ 3.7$ & $\$ 4.7$ & $4 b .3$ & 46.1 & 50.2 & 52.1 & $54 .:$ & 5 & 59.0 & ¿1.5 & 54.6 \\
\hline & & $5-01$ & $O E-0 !$ & $9 t-0 t$ & $3[-1,4]$ & (1) & $65-91$ & JE-0! & $5-01$ & $C E \sim B !$ & $9 E=01$ & ¿. Eq -01 & $1,4(-1) !$ & $1.3 E+4 \mid$ & {$[2-0)$} \\
\hline t8 & 175 & $17 !$ & 167 & 162 & $1 \equiv 8$ & 155 & 151 & 149 & 145 & 142 & 139 & 137 & 105 & $13 \hat{2}$ & 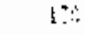 \\
\hline 43.3 & 43.5 & 43.3 & 44.5 & 65.3 & 46.4 & 47,1 & 49.2 & 50.9 & 52.7 & 54.7 & 56.9 & 59.10 & ذاذ & 63.7 & ca.? \\
\hline EE-61 & $3.0 E-01$ & $3 .: E-91$ & 2. $3 E-6 t$ & 2. IE-C! & 2. . IE-ol & $2.55=01$ & $2,4 E-01$ & I. ลE-ט́: & {$[E-d t$} & $9 E-1) !$ & $\mathrm{PE}-\mathrm{C:}$ & $5 E-91$ & 1. $+[-3) 1$ & ZE-1! & [E-ji! \\
\hline 130 & 175 & 171 & 157 & $: 64$ & 160 & 155 & 153 & 153 & 147 & 144 & $1+1$ & 159 & $: 37$ & เพ: & $: \because 3$ \\
\hline 46.7 & 46.8 & 47.6 & $\$ 7.7$ & $4 B .5$ & 49.8 & 50.6 & 52.2 & 53.7 & 5.5 & 3. & 59.3 & 61.5 & 23.7 & 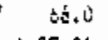 & 53.4 \\
\hline TE-01 & -18 & ;-1) & 8 & & $\varepsilon=0$ & & & & $5 E-6$ & & & $\tau$ & $3 E-13$ & IE-01 & $: E-: t\}$ \\
\hline 180 & 176 & 172 & 16 & 203 & 161 & 158 & $\ldots$ & $15 t$ & 149 & & $1 \neq J$ & $1 \div 1$ & [? & $13 \dot{s}$ & LE \\
\hline 50.7 & 0.1 & 50.4 & $\$ 1.0$ & 51.7 & $\$ 2.7$ & 5.7 & $55 . ?$ & 56.7 & Es.: & 50.1 & 52.0 & 54.0 & 36.2 & Ee.7 & +in. \\
\hline
\end{tabular}

Whole Body 0ose (rem) from Inhalation Estimated for 0000Z May 7, 1986, for the Southeast Quadrant. Bearings are in Degrees and Distances are in Miles from Chernobyl. 


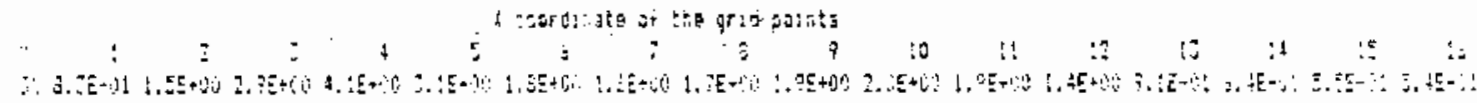

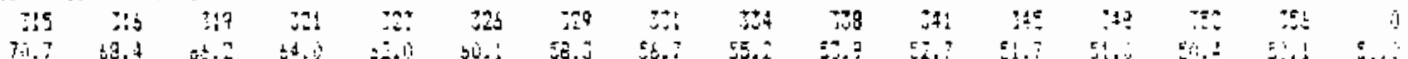

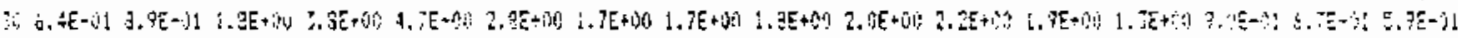

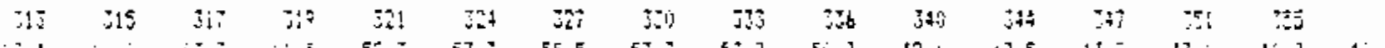

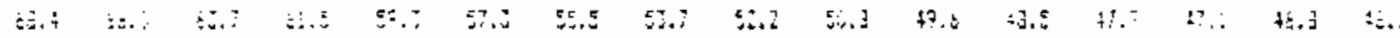
$\because$ 马.

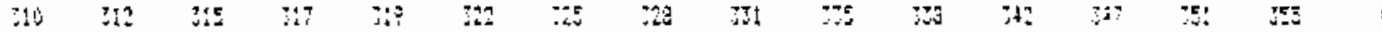

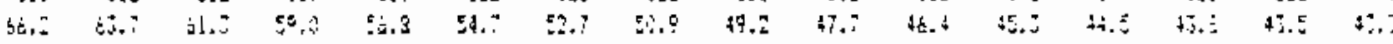

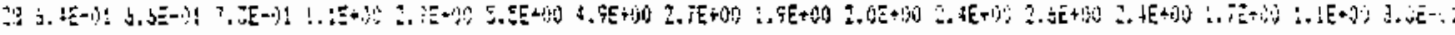

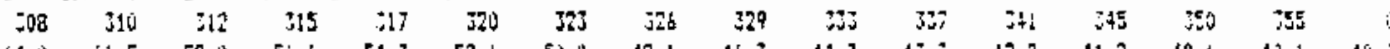

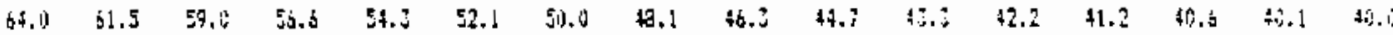

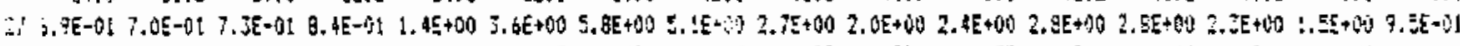

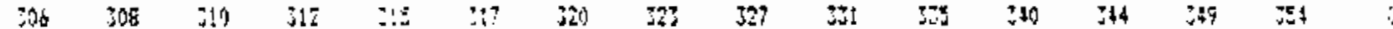

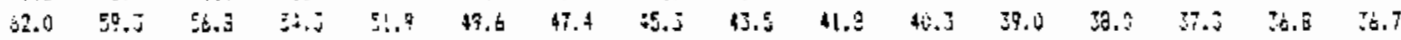

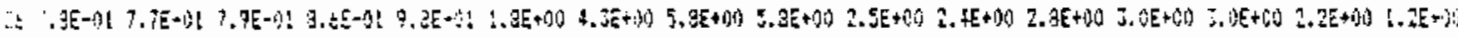

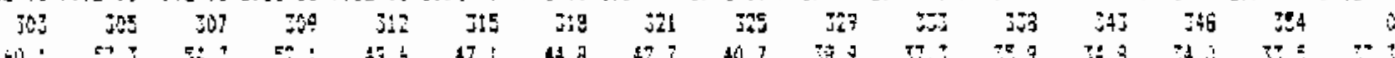

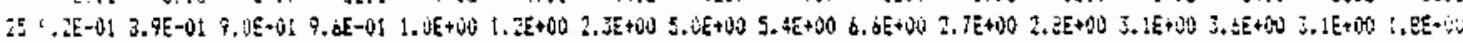

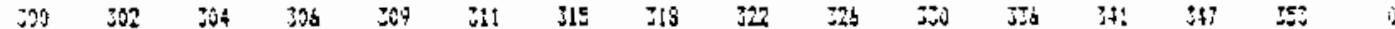

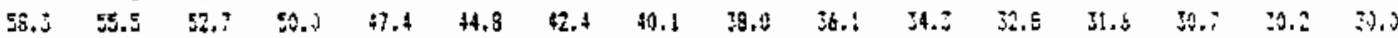

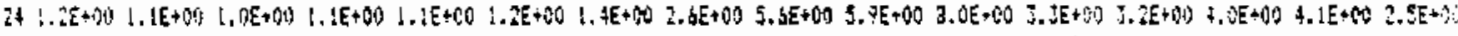

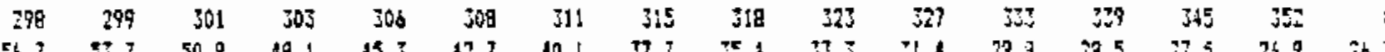

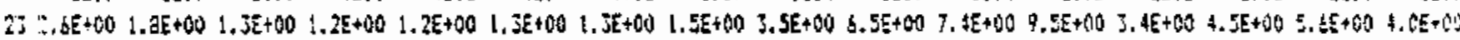
$\begin{array}{lllllllllllllll}295 & 296 & 299 & 300 & 302 & 304 & 307 & 311 & 315 & 319 & 324 & 350 & 336 & 344 & 35 !\end{array}$

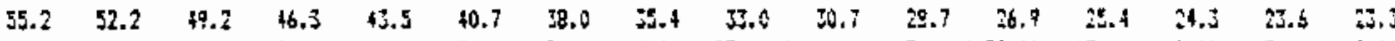

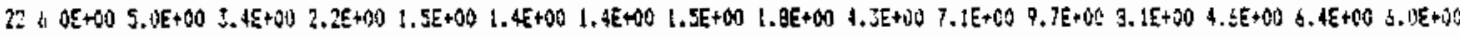

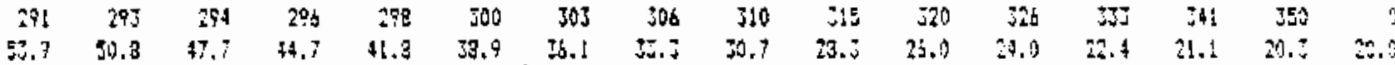

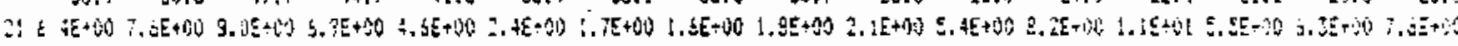

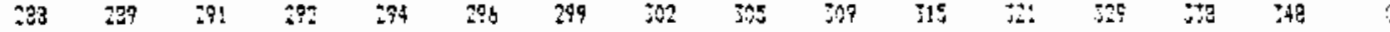

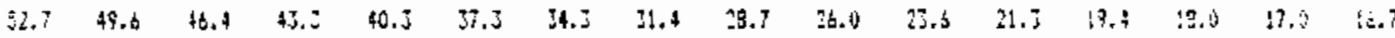

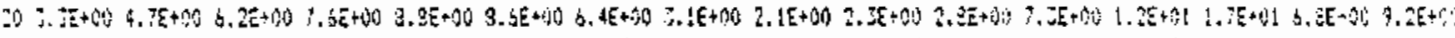

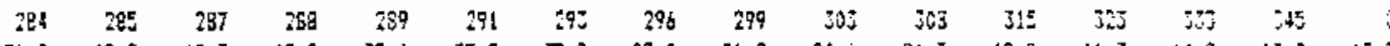

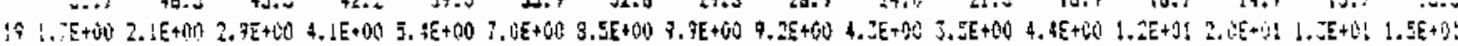

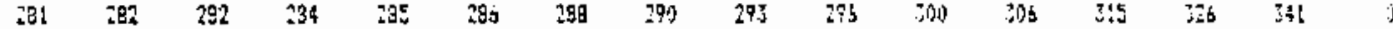

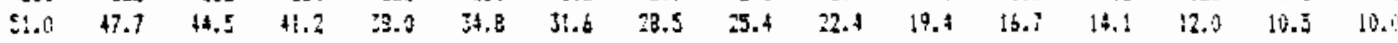

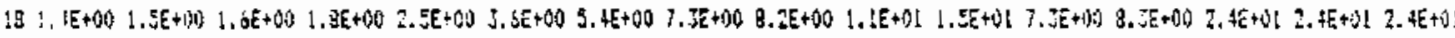

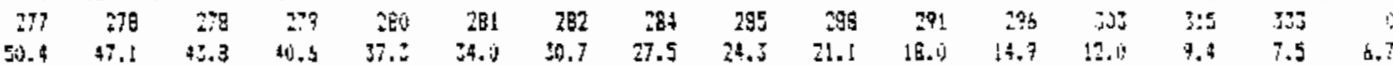

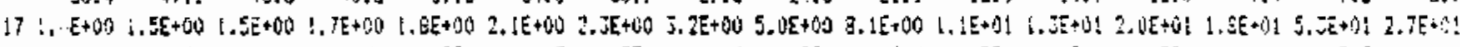

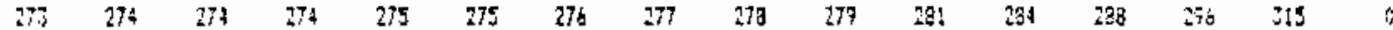

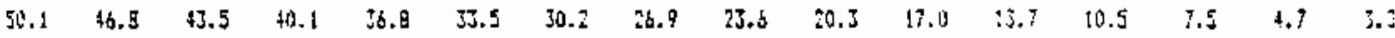

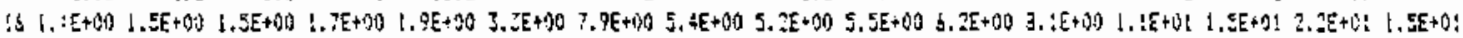

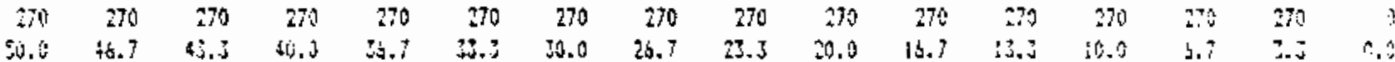

Thyroid Dose (rem) from Inhalation Estimated for 00002 May 7, 1986, for the Northwest Quadrant. Bearings are in Degrees and Distances are in Miles from Chernoby?. 


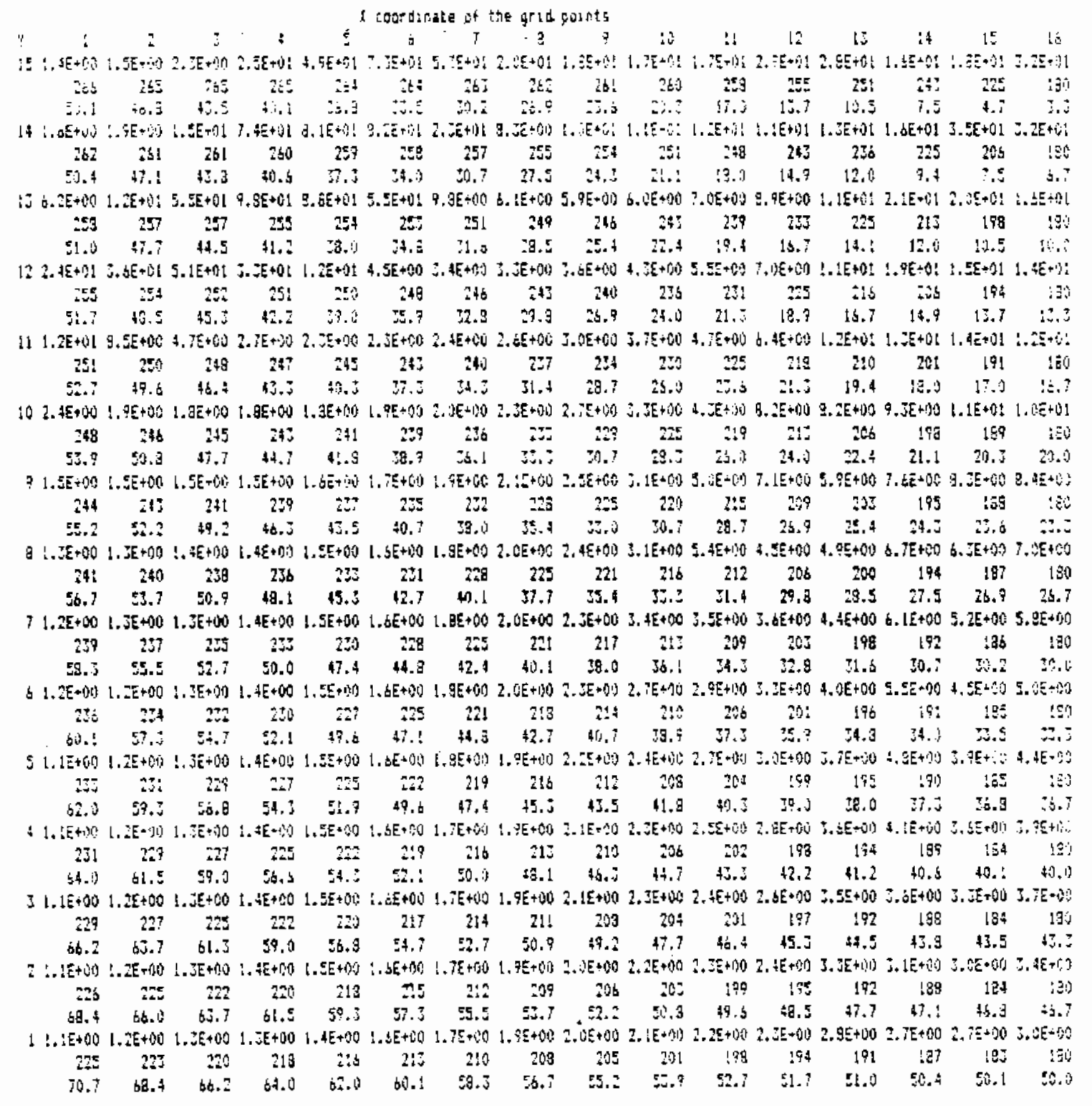

Thyroid Dose (rem) from Inhalation Estimated for $0000 z$ May 7, 1986, for the Southwest Quadrant. Bearings are in Degrees and Distances are in Miles from Chernobyl. 


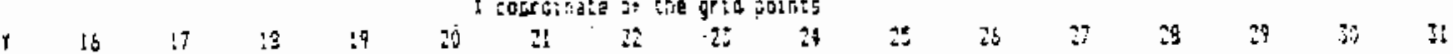

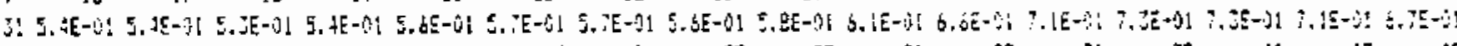

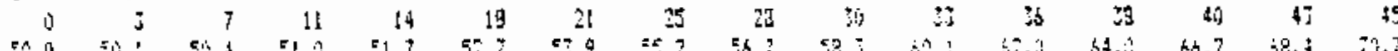

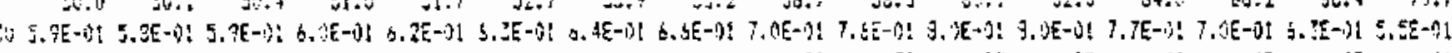

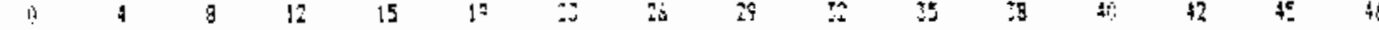

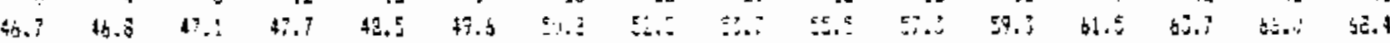

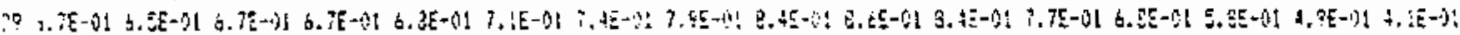

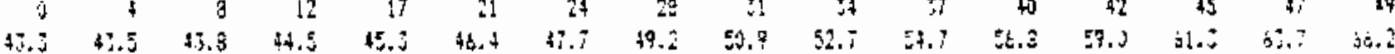

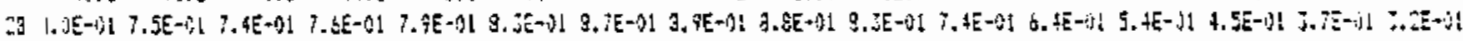

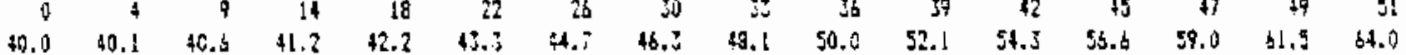

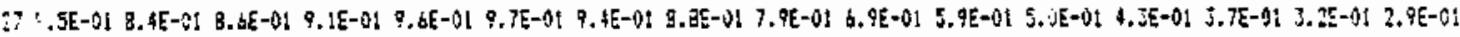

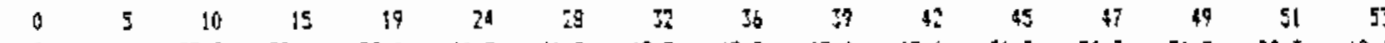

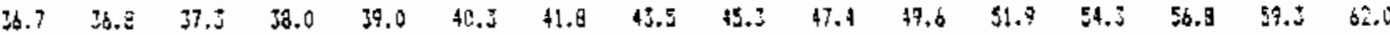

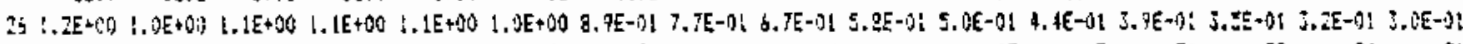

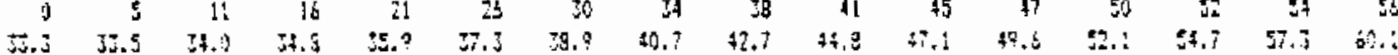

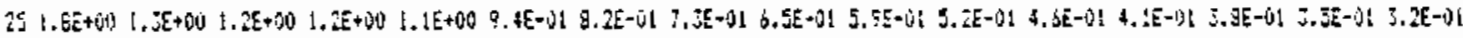

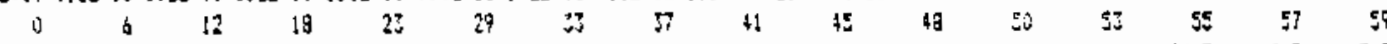

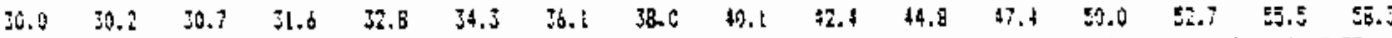

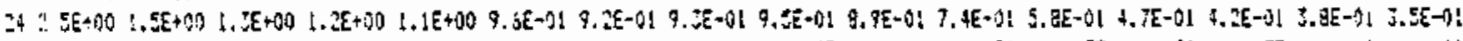

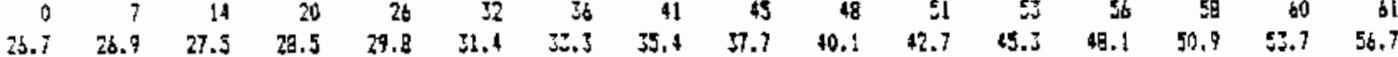

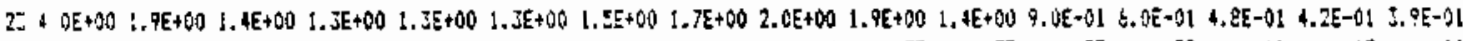

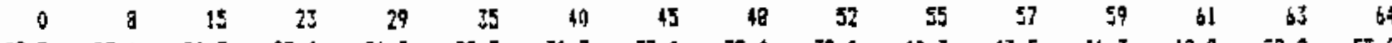

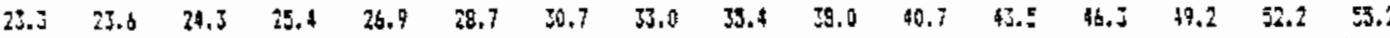

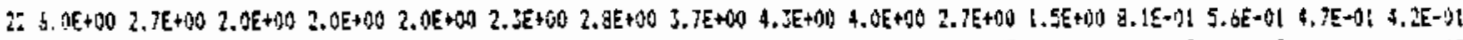

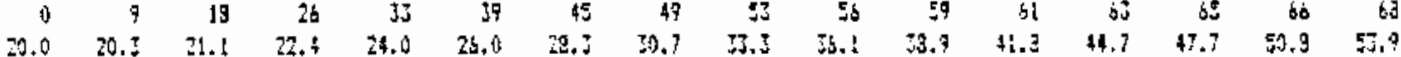

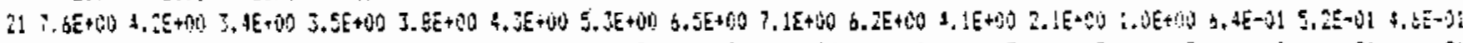

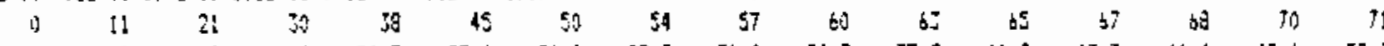

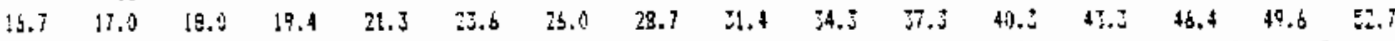

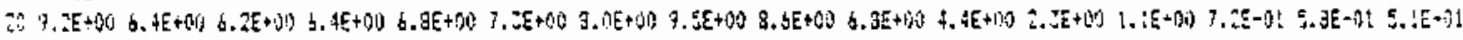

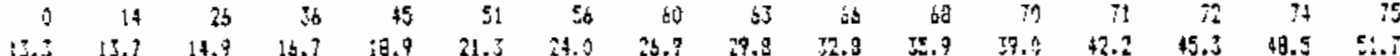

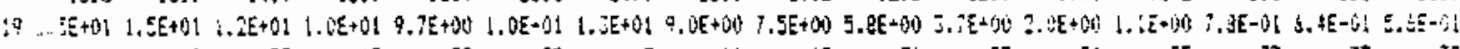

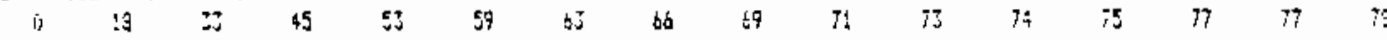

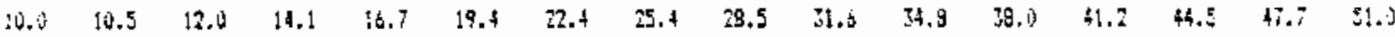

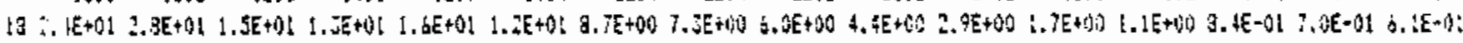

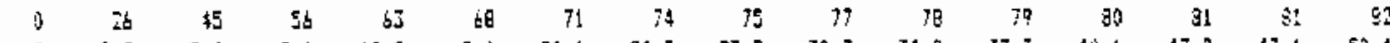

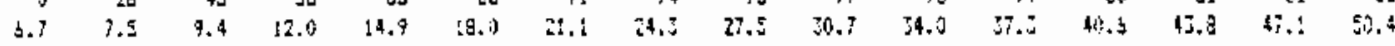

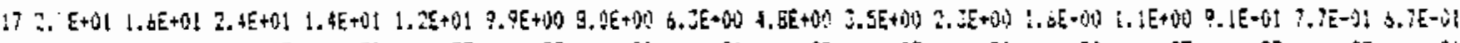

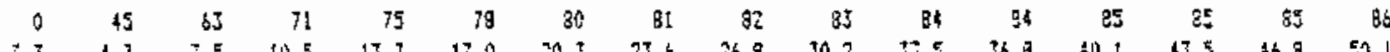

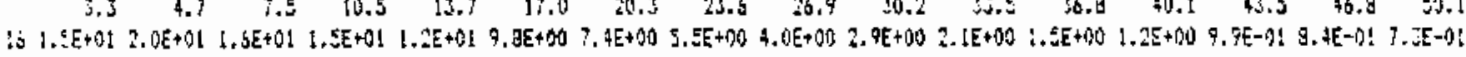

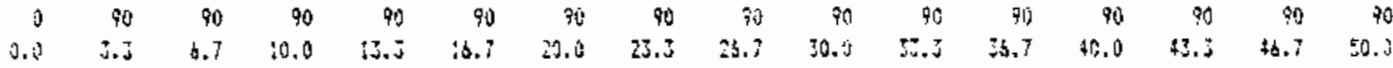

Thy roid Dose (rem) from Inhalation Estimated for 00002 May 7, 1986, for the Northeast Quadrant. Bearings are in Degrees and Distances are in Miles from Chernobyl. 


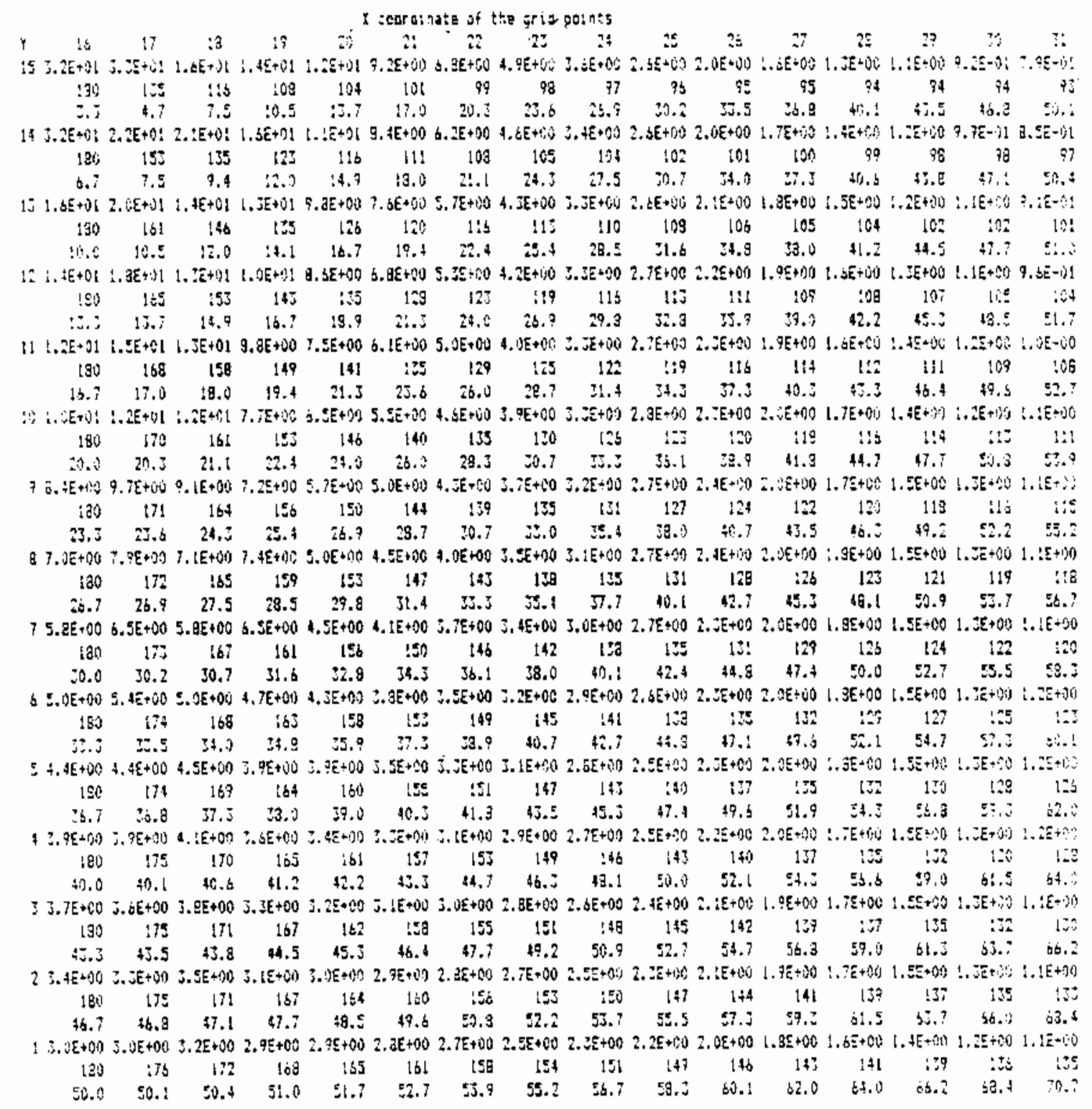

\section{Thyroid Dose (rem) from Inhalation Estimated for 00002 May 7, 1986, for the Southeast Quadrant. Bearings are in Degrees and Distances are in Miles from Chernobyl.}




\begin{tabular}{|c|c|c|c|c|c|c|c|c|c|c|c|c|c|c|c|}
\hline & & & & & & t & - & & & & & & & & \\
\hline : & 2 & : & ; & 5 & in & $i$ & $\hat{o}$. & 9 & 10 & 11 & 12 & 13 & 14 & 15 & $1 ;$ \\
\hline 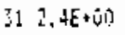 & $15+90$ & 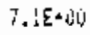 & 7. & $\therefore .55+20$ & setati & $4+(E+1)$ & $A, \vec{E}+\vec{E}+i d$ & $4 . S E+i)$ & $4 . Z E+00$ & & $3.55-(0)$ & $\therefore \Delta E+00$ & 2. $\left.i S+\hat{N}^{2}\right)$ & $\{.4 E+0\} !$ & $E+1$ \\
\hline 215 & 31s & $3: 7$ & ist & כמה & $3: 6$ & 327 & $M$ & 334 & 站 & itl & 345 & 348 & 352 & 25 & 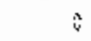 \\
\hline 70.7 & $66 . ;$ & bt.? & 34.0 & 52.8 & 60.2 & 58.3 & $5 b .7$ & 35.2 & ك. ك. & 52.7 & 51.7 & Si.人̂́ & 50.9 & $5 i .1$ & 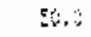 \\
\hline & & & & & & & & & & & & 1500 & E+ous & $i q+j$ & \\
\hline $1: 3$ & 315 & 315 & 319 & $32 !$ & 324 & 327 & 330 & 335 & 336 & 310 & $\$ 44$ & 347 & 35 & Es & : \\
\hline 26.4 & ob.:" & 6.5 & b1.5 & 57.2 & 57.3 & 55.5 & 53.7 & 52.2 & $5 . .8$ & 77.6 & 49.5 & $\$ 7.7$ & 47.1 & 46.3 & 45.7 \\
\hline$\therefore A E+10$ & 2. $\{\subseteq+5$ & $2.35+20$ & $5.0 E+40$ & $!+! \subseteq+60$ & $1 E+0 !$ & t. $\tilde{E} E+v($ & $4.55+00$ & $A E+150$ & $4.05+0.0$ & S. $\pm E+\infty$ & $f E+m !$ & $4,5 E=0]$ & 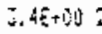 & 2.LE+QOS & $2.35+5$ \\
\hline 311 & 312 & 315 & $31 ?$ & $7: 9$ & 32 & 325 & 309 & $33 !$ & $\mathrm{XE}$ & $\because 3$ & 342 & 347 & zis! & 205 & 3 \\
\hline 6.2 & 43.7 & $\$ 1 . z$ & 59.0 & 56.3 & 54.7 & 52.7 & 50.9 & 49.2 & 17.7 & 4.4 & $\$ 5.3$ & 44.5 & 45.8 & 43.5 & 4.2 \\
\hline 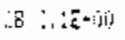 & $\therefore \therefore E+i a$ & 7.JE, di & $3,25+18 s$ & 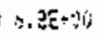 & 2. $E E+171$ & 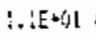 & b. $\{E+919$ & : SE $=0$ & 4. $3 E+30$ & $\Sigma, a \bar{c}+b i$ & $5 . E+5+i d 0$ & S. $. E+100$ & 1. $4 E+\infty 0$ & IE+PS & ietst: \\
\hline 243 & 30 & $\$ 12$ & Jis & 517 & 320 & 525 & 325 & 329 & 33 & זَבנ & 341 & 345 & 50 & 355 & \\
\hline 64,0 & 61.5 & 59.0 & 5.5 & Ei.s & 52.1 & 50.0 & $\$ 9.1$ & 46.2 & 4.7 & 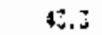 & 42.2 & $4 ! .2$ & 40.6 & 40.1 & 40.0 \\
\hline$C E+C O$ & 2 & $E=00$ & $E=00$ & $f E+80$ & 列 & $2 E+$ al & $E+01$ & $15+90$ & $A, 9 E+O C$ & $5.6 \varepsilon+00$ & $4.5 E+0 C$ & $6 . d E+\infty O=$ & $\dot{E E}+9 \hat{i})$ & $4.1 \varepsilon+\infty)$ & $15+80$ \\
\hline 306 & 396 & 310 & 312 & je & 317 & 320 & 328 & 327 & 331 & $\because 5$ & 340 & 344 & 349 & $3 E t$ & 0 \\
\hline$\pm \therefore 0$ & 59.3 & 5.3 & 54.2 & 54.9 & 49.6 & 47.4 & i5.j & 45.5 & $\$ 1.8$ & 40.3 & 29.0 & ?S. & 37.5 & 2,8 & 30.7 \\
\hline EE+1! & $2.5 E+i 0$ & $2 . b e+80$ & $2 . J \subseteq+40$ & $1,1) E+? 0$ & $4,5 E+60$ & $9.4 E+060$ & $2 E+01$ & $1.2 E+01$ & $5.7 E+60$ & $5.7 E+30$ & $6.6 E+010$ & $7.2 E+(3)\}$ & $5+40$ & $5.3 \overline{\mathrm{E}}+2)$ & 3.EE+R \\
\hline 30 & & & 309 & $3+2$ & $3 i 5$ & Jis & 321 & 125 & 争 & 323 & 320 & 345 & 343 & 353 & 1 \\
\hline$\delta j .1$ & 57.2 & 54.7 & 51.1 & 49.6 & 47.1 & 4.8 & 42.7 & 413,7 & ;a.? & $n .3$ & 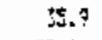 & 34.2 & 34.0 & I.s & J \\
\hline 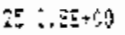 & $5+d i$ & 2.E+ & $3,6 \varepsilon+\infty)$ & $3.15+010$ & $4 E+4 i$ & $5 .+1 E \div 6$ & OE+v́! & !E+0! & VE+1)! & 5. & SE $\div 00$ & $7.3 E+100$ & $E E+\infty n$ & EE SO : & 5. $62+95$ \\
\hline $\mathrm{si}$ & 302 & int & Sos & 509 & itt & ris & 319 & 322 & 323 & 300 & 336 & 74 & it & 353 & ; \\
\hline 53.3 & 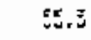 & 52.7 & 80.0 & 47.4 & 44,9 & 42.4 & 40.1 & $\$ 8.6$ & 75.1 & 34 & 2.9 & 1.6 & 20.7 & 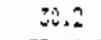 & No \\
\hline$i+n$ & $E+b ?$ & $5+1+2 x$ & 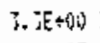 & $A E+i$ & $5 \varepsilon+39$ & $S \bar{E}+20$ & {$[4$} & 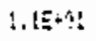 & $\Delta+\sigma_{1}$ & $1.5 E+$ & $E+10$ & - & $15+199$ & $E+\infty$ & $i=9 . j$ \\
\hline 238 & 299 & Jü! & 303 & 306 & 308 & 311 & 315 & $3: 8$ & 323 & 汭? & 30 & 539 & 345 & 552 & 0 \\
\hline 54.7 & 3.7 & 50.9 & 48.1 & 45.3 & 42.7 & to. ! & 5.7 & 85,4 & 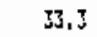 & 31.9 & 29.8 & 23.5 & 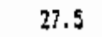 & 26.9 & 26.7 \\
\hline FE+201 & $4,4 E+00$ & $3 . \mathrm{AE}+\mathrm{OD}$ & $3.0 E+\infty)$ & $Z E+\infty 0$ & $3.9 E+00$ & $3.3 E+100$ & $4.2 E+\infty D$ & $4 E+00$ & $2 E+101$ & $1 .+2 E+01$ & $1.95+9 !$ & $7.9 E+00$ & 1. $\Delta E+0 !$ & $3 \mathbf{E}+01$ & 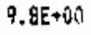 \\
\hline 27 & $27_{b}$ & 299 & 300 & 302 & 304 & 307 & $3: 1$ & 315 & 319 & 324 & 350 & is & 344 & $35 !$ & $\hat{0}$ \\
\hline 55. & 52.2 & 49.2 & 46.3 & 43.5 & 40.7 & 38.0 & 35.4 & 15.0 & \pm 0.7 & 28.7 & 26. * & 25.4 & 24.3 & 23.5 & 25.3 \\
\hline $1 \mathrm{IE}+\mathrm{DI}$ & $9.7 E+00$ & $7.25+10$ & 5. $2 E+100$ & $2 E+0 D$ & $1 E+130$ & $2 E+00$ & $A E+\infty 0$ & $15+\infty$ & $8+00$ & $E+0 !$ & $E+O I$ & EEt+1) & $E+0$ & $E E+01$ & $\varepsilon+1 !$ \\
\hline $29 !$ & 993 & 294 & $70 \%$ & 290 & 300 & 305 & 306 & 310 & 315 & 30 & 328 & 33 & 3 & 350 & 0 \\
\hline 5.9 & 50.8 & 47.7 & 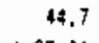 & 41.8 & 38.9 & 35.1 & 3.2 & 30.7 & 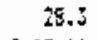 & 2 & 24.9 & 22.4 & $2 ! .1$ & 20.7 & 20.0 \\
\hline Fin: & tol. & 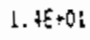 & 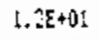 & $\Delta E+00$ & $3 E+00$ & $50+00$ & $85+60$ & $E+0 \hat{\theta}$ & $3 E$ & E. & $(5 E+1) !$ & $25+0$ & te & $=E+21$ & {$[<+01$} \\
\hline$: \Xi$ & 239 & $\hat{\imath} \hat{Y} !$ & & 574 & 294 & 399 & $\min 2$ & . 5 & 30 & II & $j 21$ & ;a? & xa & 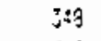 & j \\
\hline 52 & 99.6 & 46.4 & 4 & 40.3 & 32.5 & 34.3 & Ii.t & 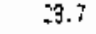 & " & 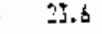 & 21.3 & .4 & .8 & 57.0 & L. 7 \\
\hline & 40 & & 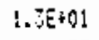 & +0 & 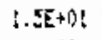 & $=$ & E+Q & $E+10$ & 6. $\$ E+\$ 30$ & $5 E+$ & $E=01$ & $E+01$ & $E+1):$ & $4=+9 !$ & $E+01$ \\
\hline 75 & 29 & 25 & & $2 \div 9$ & 391 & 293 & 396 & 299 & 303 & 3 & jis & in & 3 & $j 4$ & 4 \\
\hline 51.7 & 48.5 & $4=2.3$ & 42.2 & 83 & TE.? & 32.3 & 29.8 & 23.7 & 24.0 & 21.3 & 15.9 & : 1.7 & $: 9.3$ & $: 2.7$ & 15.3 \\
\hline $5+00$ & 5. $\{\{+1\} ?$ & a. 洰我 & 8. $25+50$ & $E+4 ! !$ & $E+13 t$ & $1.5 E+01$ & $1.7 E+31$ & [. $1 E+01$ & $2+00$ & $9.25+60$ & $E \div 01$ & $E+0:$ & $3,05+8$ & $2.9 E+2 t$ & $E+1$ \\
\hline $75 !$ & 9 & $=97$ & & 295 & 296 & 248 & ?70 & 29 & 296 & To & :16 & & 86 & 341 & 3 \\
\hline 51.3 & 47.7 & 44.5 & $4 ! .2$ & 30.2 & 34.3 & 31.6 & 23.5 & 25.4 & 22.9 & 14. & is. 7 & 13.1 & $: 2.0$ & 10.5 & 10.0 \\
\hline$E+10$ & $3 E+10$ & $(E+N)$ & {$[2 x+3]$} & $5+94$ & $9 E+i: 0$ & $\mid E+105$ & EE+i) & $5 \mathrm{~F}$ & & EE+ & $\Delta[-D !$ & Eet & 5 & 8 & $a+9$ \\
\hline 27 & & & & $m+a$ & $M A i$ & 987 & 704 & & & & & & & & \\
\hline$\varepsilon ;$ & & & & 3.2 & 54,0 & 30.7 & 27.5 & & & 13.2 & 14.9 & 12.0 & 9.4 & S... & .7 \\
\hline & & 0 & 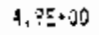 & $0 E .2 E+\hat{O}$ & $\pm . E E+10$ & $E+b)$ & $3.9 E+40$ & $E+01$ & th & $2.25-01$ & $\hat{2} .5 \pm+0 !$ & $3+\Delta E+111$ & Z. $35+01$ & A. $J E+0 !$ & $E,\{E+\})$ \\
\hline 27 & 27 & 274 & 174 & 279 & 175 & $27 b$ & $27 ?$ & 278 & 279 & $23 \mathrm{E}$ & 234 & 3. & $b$ & 515 & 0 \\
\hline 50.1 & 46.9 & 43.5 & 40.! & 36.5 & 5.5 & 30.2 & 26.9 & 23.6 & 26.2 & 17.0 & 13.7 & 10.5 & 7.5 & 4.7 & 3.2 \\
\hline$E+00$ & $4 E+20$ & $7 E+100$ & $A E+O D$ & $\varepsilon+00$ & $\{E+\infty)$ & $5 E+60$ & $\{E+00$ & {$[+3)$ L } & & $t E+13 !$ & $0 E+19 !$ & $b=+1$ & $E+01$ & $E+01$ & $+19 !$ \\
\hline$? 7$ & 27 & 0. & & tov & 270 & 270 & S & 80 & & & Ste & 27 & & 70 & \\
\hline 50. & 46.2 & 43.5 & $46 . j$ & Tंड.: & ن. & 30.0 & 26.7 & 23.2 & 9.8 & 16.7 & 12.3 & (1). & b. 7 & j.j & .0 \\
\hline
\end{tabular}

\section{Lung Dose (rem) from Inhalation Estimated for $0000 z$ May 7, 1986, for the Northwest Quadrant. Bearings are in Degrees and Distances are in Miles from Chernobyt.}




\begin{tabular}{|c|c|c|c|c|c|c|c|c|c|c|c|c|c|c|c|}
\hline & & & & & & & & & & & & & & & \\
\hline : & 2 & ; & 7 & $\vdots$ & $\varepsilon$ & 7 & $?$ & 9 & :0 & $\therefore:$ & :? & 15 & $!$ & !c & 16 \\
\hline & & Erol & $j E+i c$ & $E+i_{i}$ & TE+? & $E+B \mid$ & $E *$ & $\lfloor .5 E * 01$ & 1. $3 \leq-3\}$ & $E+$ & $E+i$ & $75+t$ & Et & 5 & Ex+: \\
\hline 850 & 265 & 35 & 265 & 767 & $26 t$ & $26:$ & 262 & 31 & 260 & 259 & 258 & $2=1$ & 243 & $2 \div 5$ & $: \$ j_{j}$ \\
\hline 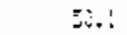 & 46.2 & $4 \% .5$ & 40.1 & 3.5 & 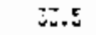 & 3.2 & 26.9 & $\approx \pi, b$ & 2 & 17.6 & 13.7 & 14.5 & 3.5 & 4.7 & $\therefore$ \\
\hline 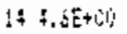 & 5.'2E+100 & B. $5 E+19$ & as & Z.. & 2 & 1.55 & $1.5 E+01$ & 1. $g \varepsilon+1 j !$ & $2 . ! 5+! ! !$ & $2,+E+6 !$ & $2.5 E+1) !$ & $3 .(\varepsilon+1):$ & $\therefore .7 E+11$ & 7. $\mathrm{X} E+3 !$ & bE= $=1 ?$ \\
\hline ch? & 351 & $i s 1$ & 260 & 259 & 253 & 257 & 255 & 84 & הE: & 246 & 243 & 236 & 1.5 & 205 & 180 \\
\hline 80.4 & 47.1 & 8.3 & 40.5 & כ. & 34.0 & 30.7 & 27.5 & 24.3 & 21.1 & $13 . s_{1}^{4}$ & 59.7 & (2.) & 2.7 & ?.5 & 5.". \\
\hline & $\mathrm{E}+$ & $E+9 t$ & & . & $5-0$ & 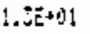 & $5+0$ & $E+21$ & 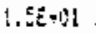 & {$[+1$} & 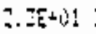 & ;et; & $5+0 !$ & 5 & {$[x+1)$} \\
\hline 200 & 257 & 257 & 255 & 254 & J J & 751 & 249 & $2+6$ & 343 & 239 & 23 & 225 & 215 & 198 & 120 \\
\hline 51.3 & $\$ 7.7$ & 44.5 & $\$ 1 .=$ & 洁. & 活. & $3 ! .6$ & 23.5 & 25.4 & 54 & $\mathfrak{i}, 4$ & 10,7 & 11.1 & $12 . j$ & 14.5 & $\therefore$. \\
\hline$E+j$ & IE+ !1 & (., $T E+1\}:$ & 1. AE+1)! & $E+8 \pi$ & S.SE+ONI & $3 . \$ E=09$ & $\left.9.2 E+i_{i}\right]$ & $1, Q E+v i$ & $35+0 !$ & $! . S E+0 !$ & 1. $.5+01$ & ?E+4)! & 5. $\varepsilon[+0:$ & $4.42+6 i$ & $4 . \Omega \varepsilon+\dot{v} !$ \\
\hline 255 & 754 & 252 & $2 \varepsilon_{1}$ & ניוֹ & $2+6$ & 245 & 243 & 343 & : & $2:$ & $7 n z$ & ל! & 286 & 194 & ! \\
\hline 51.7 & 43.5 & 45.2 & 42.2 & 37.0 & 35.9 & 2.3 & 39.3 & 25.7 & 24.2 & $2: .3$ & 13.9 & $: \varepsilon .7$ & 14.9 & 15.7 & 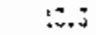 \\
\hline $7.3 E+31)$ & $1,2 E+30$ & d. $7 E+j U$ & b.SE+0ro & $6.7 E+100$ & $8.9 E+40$ & $7.4 \varepsilon+1), j$ & 8. $\pm E+0 ?$ & : & $2 E+8 \mid$ & $.4 E+1: i$ & 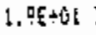 & $\mathrm{FE}+\mathrm{i} 1$ & S. $F E+01$ & 3., & 3.es+: \\
\hline$A E:$ & 250 & 248 & 247 & 245 & 247 & 240 & דיב & 124 & 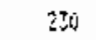 & $2 n$ & 219 & 211) & $30:$ & 171 & $\because 3$ \\
\hline 52.7 & 49.1 & 46.4 & .3 & 49.: & 3.3 & 3.3 & 31.4 & 3.3 & 75.18 & Zsos & $2: .3$ & 19.9 & 9.2 & 17.2 & !...? \\
\hline $5.3 E+C D$ & $5.4 E+100$ & $5 E+100$ & $E E+00 \mathrm{~S}$ & $1 E+50$ & $4 E+\Delta O$ & $2 E+30$ & $8 E+10$ & $1 E+20:$ & $\{E+B !$ & EF+? & $E[-1)$ ! & $E E+n 1$ & $3 E+2 t$ & EE+1)! & iE +0$\}$ \\
\hline $2 \div 8$ & 216 & 245 & 243 & $24:$ & 79 & $2 z$ & 203 & 227 & 725 & 219 & 13 & $2 t$ & 102 & 99 & 130 \\
\hline 5.7 & 90.3 & 4.7 & $\$ 4.7$ & : $: .9$ & 38.9 & 35.1 & $3 . .3$ & 30.7 & I... & $36.1)$ & 24.17 & 23.4 & $21 . !$ & 30.5 & $2 \hat{i}, j$ \\
\hline $9+\$ 0$ & $i \bar{i}+j \bar{j}$ & $E+C O$ & $E+003$ & $E+6 u$ & $E+\infty 0$ & $E+C B$ & $E+40$ & $E+\$ 0 ：$ & $1 E+? ! 1$ & $t E+A$ & $\$ E+01$ & $85-0 !$ & $\mathrm{EE}+1 !$ & $E E+0 !$ & EE+4! \\
\hline $2 \div 4$ & $24:$ & $24 !$ & 237 & די & 275 & 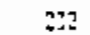 & 213 & 269 & 220 & $2: 5$ & 209 & $0 \vec{i}$ & 195 & !9a & $! 3 ;$ \\
\hline$\varepsilon z$ & 52.2 & 19.2 & $4 \delta, ;$ & 47.8 & 40.7 & $\pi .0$ & 85.4 & J.j.j & 50.3 & 3.7 & 26.7 & 25.4 & 24.5 & 25.6 & $2: 2$ \\
\hline$E+B D$ & $E+10$ & $x E+\infty 0$ & $5.5 E+C O S$ & S.REr0O & $E E+c, E$ & $1 E+190$ & $65+0,3$ & $E+C 0 !$ & $E+101$ & $8 E+\cap 1$ & EE+lit & $E E+0 !$ & $1 E+0 !$ & OE+14: & $2.3 E+96$ \\
\hline $2 * 1$ & 240 & 258 & 236 & 230 & 231 & 228 & 175 & 221 & 6 & 215 & 206 & 200 & 94 & 167 & 185 \\
\hline 56.7 & 35.7 & 50.7 & $49 . !$ & 45.3 & +2.7 & 40.1 & 37.7 & $5 * 4$ & 3 & $3: 4$ & 70.8 & 28.8 & 27.5 & 26.9 & 26.7 \\
\hline $6 E+90$ & $4.9 E+00$ & $E+\infty 0$ & $8 E+00$ & $E E+00$ & $6 . j E+00$ & d. $.5 E+100$ & $7,\{E+00$ & $E+40$ & $E+0$ & ¿. $.2 E+01$ & $2 E+i j !$ & $E+0 !$ & 1. $. F E+01$ & $1.7 E+e t$ & 1. $Q E+v 1$ \\
\hline 25 & 18 & 235 & 230 & 270 & 223 & 225 & $22 i$ & $2: 7$ & 215 & 1007 & $20 z$ & $\operatorname{lgg}$ & 192 & !ab & 100 \\
\hline 58.3 & 55.5 & 52.7 & $\$ 0.0$ & 47.4 & 74.8 & 42.4 & 40.1 & 58.0 & 36.1 & 34.3 & 32.9 & T1.6 & 0.7 & 30.2 & 20.2 \\
\hline $\mathrm{x}^{2}+[1]$ & $g E+m i$ & $5+20$ & 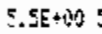 & 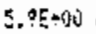 & $E+\hat{\infty} 0$ & $E+100$ & $8 E+2) i$ & $E+009$ & $9.7 E+150$ & $D E+4$ & IE+? & $z E+$ & $g E+C$ & $E E+0 !$ & $5 \bar{E}+c 1$ \\
\hline 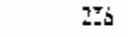 & $2 ? 4$ & 232 & 230 & $23 i$ & 225 & $22 !$ & 219 & $: \because 4$ & $21 \%$ & 206 & $20:$ & $: 95$ & is: & !ล̄ & $: 5 \%$ \\
\hline 80.1 & 57.3 & 54,7 & $52 . !$ & 43.6 & 4,8 & 44.8 & 22.7 & $\$ 0.7$ & 3.7 & 57.8 & 35.7 & $2: 9$ & $24 . j$ & J.E & 3.8 \\
\hline $5+10$ & 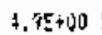 & $E+\infty 0$ & $=80$ & $E+10$ & $E+\infty 0$ & 6.7E+50 & $7,2 E+80$ & $2.0 E+\infty 0$ & S.AE-U: & $3.6 \bar{E}+00$ & $: E+01$ & $25+51$ & $1.6 E+W 1$ & 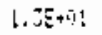 & $1,4 E+1\} 1$ \\
\hline 22 & 25 & $2 \pi 9$ & $2 n$ & ת & בan & 217 & $2: 5$ & $2 i 2$ & 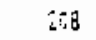 & 324 & 179 & $: 75$ & $180)$ & $: 95$ & 158 \\
\hline 62.0 & 59.3 & 56.3 & 54.3 & 51.9 & 49.6 & 47.4 & $\$ 5.7$ & 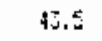 & 41.3 & sù.J & 39.12 & II. & 27.3 & צ..9 & $\therefore .7$ \\
\hline$(E+1,1)$ & $9 E+00$ & $5.25+c 0$ & $E+0 i$ & $E+1 ; i, i)$ & 8. $2 E+90$ & $E+1,0$ & $7, \mathrm{NE}+00$ & $E+i f t i$ & $\theta .6 E+69$ & $\overline{i z}+i^{\prime \prime 11)}$ & ET: & L.IE+0! & $+\varepsilon+1) !$ & $E+c i$ & $2 E+04$ \\
\hline $2 \pm 1$ & 2 & $22 ?$ & $2 n=$ & $2 n 2$ & 219 & $2 ! 6$ & $21 ?$ & 210 & 106 & 292 & 198 & 194 & 189 & 154 & $: 30$ \\
\hline 59.6 & 81.5 & 59.0 & 5.5 & 54.3 & \$2.:! & 50.3 & 48.1 & 46.3 & $44 . ;$ & 4.3 & 42.2 & 11.2 & 40. & 40.: & $40.0^{3}$ \\
\hline+80 & $7 e+60$ & $\xi+0$ & & $E E+\infty O$ & [t+ & $76+00$ & $3 E+60$ & $G E+20$ & $A E+Q 0$ & $0 \subseteq+00$ & $9.4 E+100$ & $x+v+1$ & $\{.25+0 !$ & 1. $\{\subseteq+u\}$ & $\{.2 E+0 \mid$ \\
\hline 24 & 27 & 275 & 222 & 2.20 & 217 & 214 & 3 & 709 & 204 & 201 & $\$ 97$ & 192 & :39 & $1 \mathrm{E} 4$ & (3) \\
\hline 5.2 & 63.7 & 61.3 & 59. & 5.8 & 54.7 & 51.7 & 50.9 & 49.2 & 6.7 & 46.9 & 15.: & 44.5 & 4.5 & 43.5 & 45.2 \\
\hline$E+$ & Den & & & $E+i j 0$ & $E E+00$ & $E+00$ & $2 \varepsilon+10$ & E+at) & B. $1 \hat{a}+; ; j$ & 9. SE- & $8.9 E+000$ & $1 .: 5+0 !$ & $1 . ! E+4 !$ & $\therefore, E+B !$ & [..ZET⿳亠丷厂 1 \\
\hline 39 & 32 & 227 & 220 & $2: 8$ & 215 & 212 & 209 & 205 & 205 & $19 \%$ & 180 & $: ; 72$ & 158 & :\$4 & 180 \\
\hline 53.4 & 56.0 & 65.7 & 61.5 & 59.3 & 57.3 & 58.5 & 53.7 & 5.2 & $5 ! .3$ & 99.6 & 49.5 & $\$ 7.7$ & 47.1 & 46.8 & $16 .:$ \\
\hline & Eft & $E+\infty$ & +605 & $E+\infty 0$ & $\mathrm{~F}+0 \mathrm{O}$ & $E+130$ & $15+90$ & $E+\infty 0 ?$ & $B E=0$ & $E=0$ & $6 E+60$ & $E$ & $i E+i$ & $E E+\infty$ & $5+01$ \\
\hline 225 & $22 j$ & 220 & 213 & 24 & 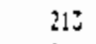 & 210 & 208 & 265 & 10 & 193 & :P4 & 27 & 137 & $: \notin 3$ & 190 \\
\hline 70.7 & ف8.4 & $8 b .2$ & 54.0 & 52.0 & so.1 & 58.3 & 56.7 & 55.2 & 53.9 & 5?.7 & $5 ! .7$ & 51.2 & 50.4 & 50.1 & 5ㅔㅔ, 2 \\
\hline
\end{tabular}

Lung Dose (rem) from Inhalation Estimated for 00002 May 7, 1986, for the Southwest Quadrant. Bearings are in Degrees and Distances are in Miles from Chernobyl. 


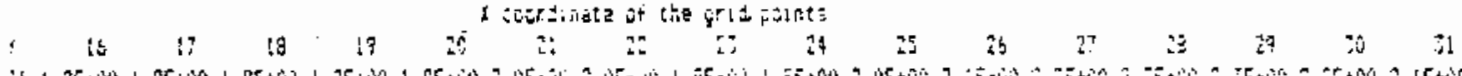

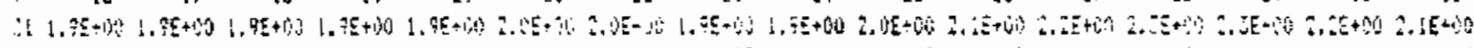

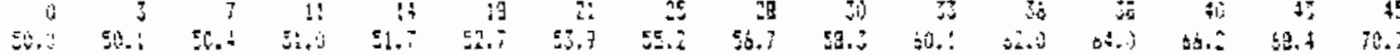

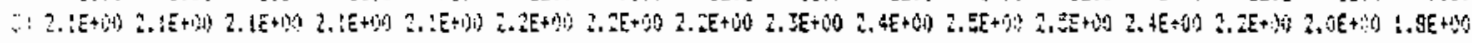

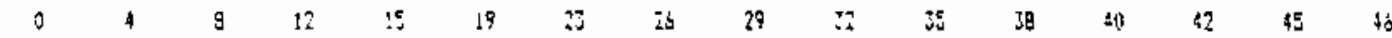

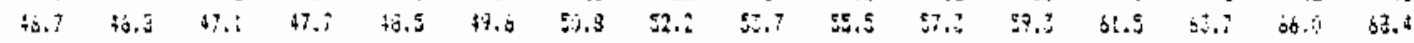

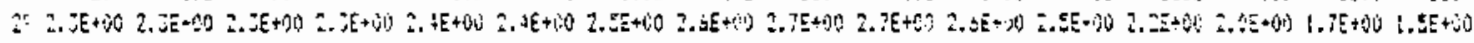

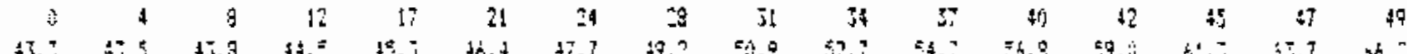

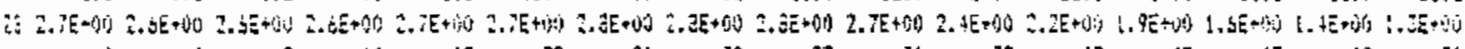

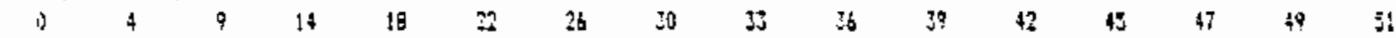

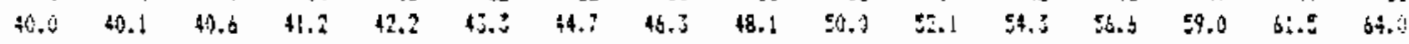

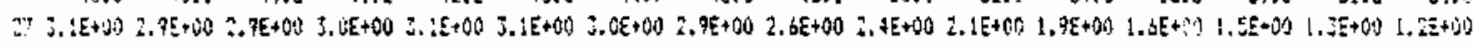

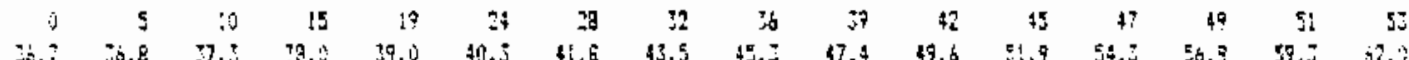

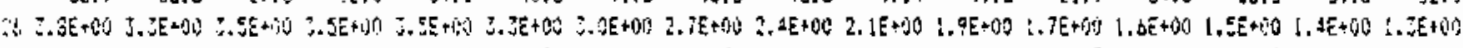

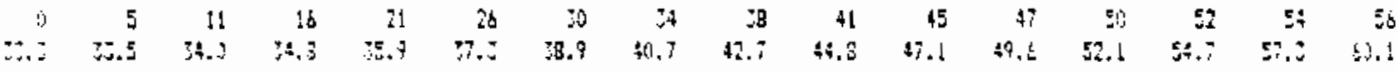

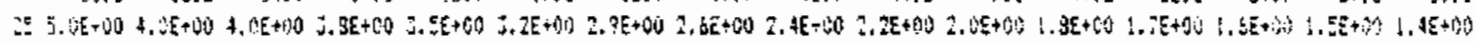

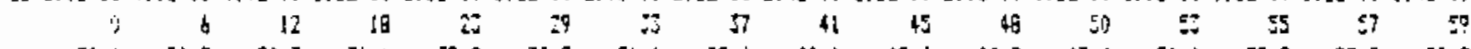

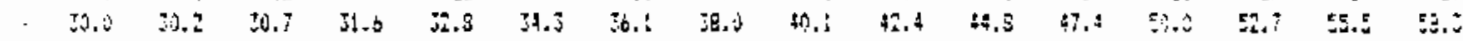

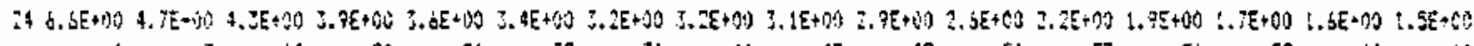

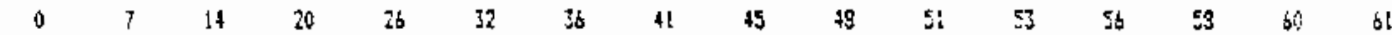

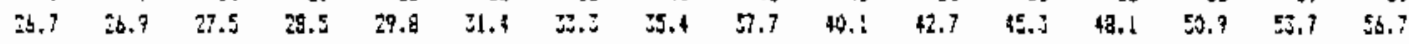

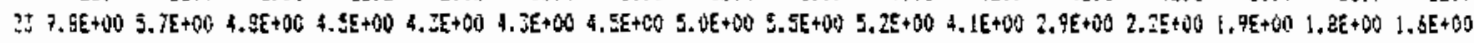

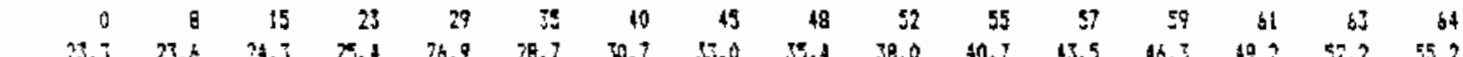

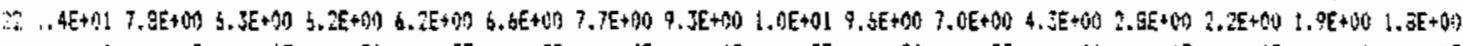

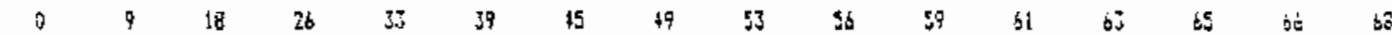

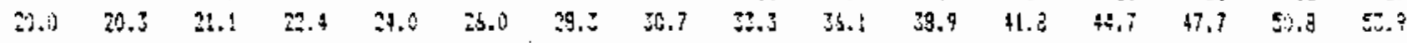

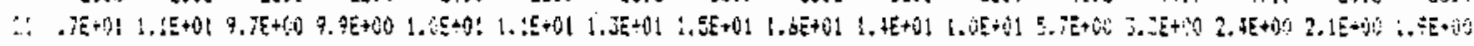

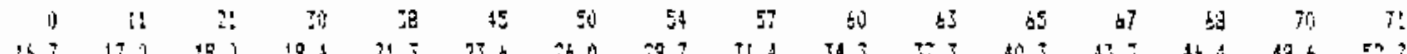

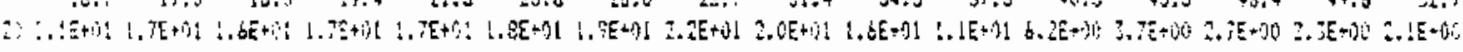

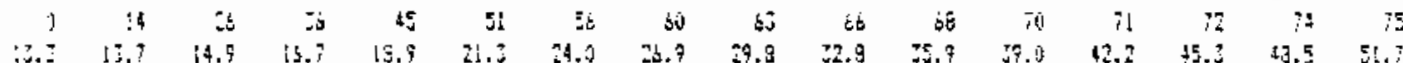

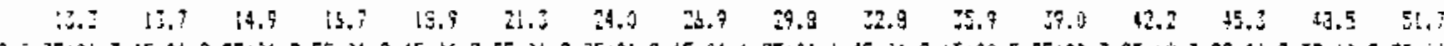

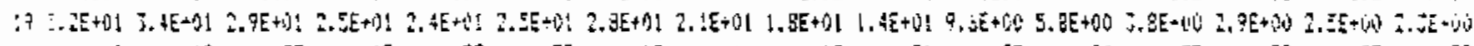

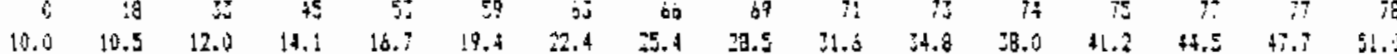

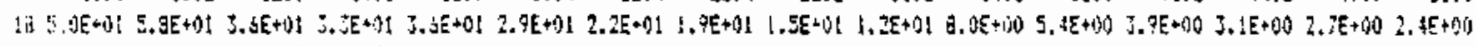

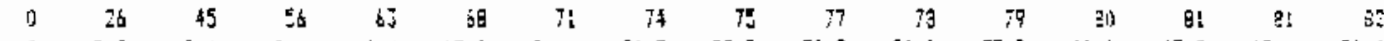

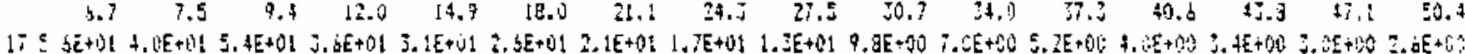

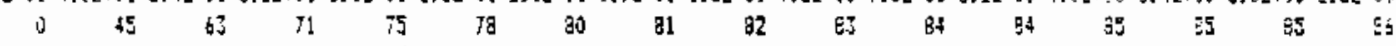

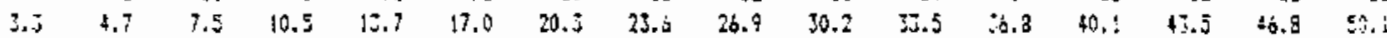

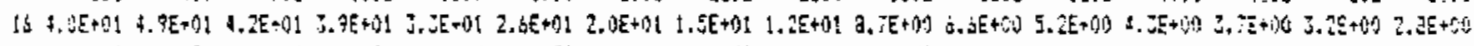

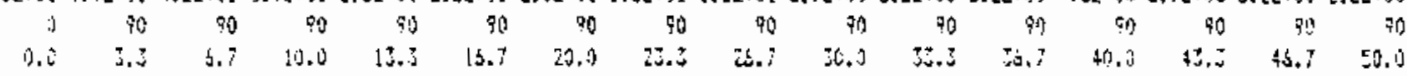

Lung Dose (rem) from Inhalation Estimated for 00002 May 7, 1986, for the Northeast Quadrant. Bearings are in Degrees and Distances are in Miles from Chernobyl. 


\begin{tabular}{|c|c|c|c|c|c|c|c|c|c|c|c|c|c|c|c|}
\hline & & & & & & $=0$ & $g:$ & & & & & & & & \\
\hline it & $1 ?$ & 12 & ¿? & 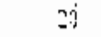 & 21 & 22 & $\because$ & it & $\approx \Sigma$ & $i t$ & 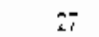 & 23 & 27 & $\pi$ & $\therefore$ \\
\hline & & & & & & & & $E=?$ & Exis & 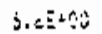 & $E+1$ & 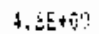 & $5+\infty$ & 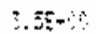 & {$[E+6$} \\
\hline 130 & 1J5 & $11 \%$ & 108 & 1.94 & 91 & 93 & 79 & 27 & io & 95 & 35 & 74 & 94 & 94 & \$: \\
\hline 3.3 & 4.7 & $\therefore .5$ & i⿱口. $z$ & 15.7 & 17.0 & 20.3 & $\because .3$ & 25.9 & 33.2 & $\because .5$ & 36.6 & 40.1 & 43.5 & 40.9 & 5. \\
\hline 1.6E+01 & 5. $5 E+0:$ & $5 . J E+7$ & $. j E+\eta$ & i)E+111 & $2 .+[2+9 !$ & $1.8 E+01$ & $1 .+E+8$ & $\lfloor,(E+1) !$ & $3 .+c+00$ & $5,9 E-00$ & $5.9 E+60$ & $4.7 E+Q U$ & $1.3 E+\hat{y} 0$ & $\therefore .7 E+D D F$ & $3.35+169$ \\
\hline 180 & 153 & 135 & 123 & 116 & 111 & 100 & 105 & 104 & 102 & 101 & 160 & 99 & $9 B$ & 72 & $\hat{3} ?$ \\
\hline 6.7 & $i .5$ & 4 & 12.6 & 14.9 & (3.1) & 21.1 & 24.2 & 37.5 & 20.7 & 34.0 & 3.2 & 10.6 & $4.3,3$ & 47.1 & 5i, 4 \\
\hline$x+2+12$ & $3.0 k+4$ & qE+U! & $s[+0]$ & $i \varepsilon+0 !$ & 2. $.2 E+01$ & 1. $.7 E+0 R$ & $1.35+01$ & $1.2 E+0 !$ & $5.1 E+C 0$ & $1.2 \mathrm{E}+\mathrm{jO}$ & b. [E+(I) & $5.3 E+00$ & $\left\{. \Delta E+t^{t} \|\right.$ & 4. OE+ 00 & $2.5 \mathrm{E}+[, 0]$ \\
\hline 160 & 161 & 146 & 125 & 26 & 160 & 116 & 113 & 116 & 133 & 105 & 05 & 104 & 102 & 102 & $: 01$ \\
\hline 10.0 & 10.5 & 13.0 & 14.1 & 96.7 & 19.4 & 22.4 & 35.7 & 22.5 & ri.s & $j 1.9$ & 39.0 & 41.2 & 44.5 & $\$ 7.1$ & E!..? \\
\hline & $4.35+01$ & & $E+1$ & 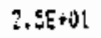 & $E+0 !$ & $6 E+Q !$ & JE+1) & $1 E+0 !$ & S. $5 E+1) 0$ & $7.65+00$ & E.SEE+0U & $3.5 E+00$ & $4.3 E+9)$ & 4. $2 E+\hat{p} 9$ & SETOS \\
\hline 120 & 105 & 153 & $14 J$ & 135 & $1 z$ & 123 & $11^{9}$ & i16 & $1: j$ & $! ! !$ & 109 & $10 E$ & 107 & 108 & 14 \\
\hline 13.3 & 13.7 & 14.9 & 16.7 & 15.9 & 21.3 & 24.0 & 25.9 & 39.8 & $3 n .3$ & 55.9 & 39.0 & 42.2 & 45.3 & 49.5 & 51.7 \\
\hline $3.5 E+01$ & 4. $0 E+5]$ & $3.55+c 1$ & $6 \overline{\bar{c}}+01$ & $2 E+01$ & $.35 \div 01$ & 1.SE+U1! & $1 . J E+00 t$ & $.|E+i| l \mid$ & $9.7 f+00$ & $7 . \hat{A E}+\$ 0$ & $8.7 \varepsilon+00$ & S. $\Xi E+60$ & S. $0 E+90$ & $4 E+20$ & $3.2 E+40$ \\
\hline 180 & 169 & 155 & $: 49$ & 141 & $1: 3$ & 129 & 125 & 122 & $1: 9$ & 116 & 114 & 112 & $11 t$ & 107 & $: 03$ \\
\hline 16.7 & 17.0 & 80.0 & 4. & 21.3 & 23.5 & 26.0 & $25 . ?$ & 31.4 & $\therefore .3$ & 5.3 & $\$ 0.3$ & 43.3 & 46.1 & 49.5 & $5 \approx .5$ \\
\hline $0 E+f !$ & $5+[E+01$ & $\varepsilon+\hat{Q} !$ & $E[001$ & QE+o! & $7 E+91$ & $1.5 E+0 t$ & $2 E+n 1$ & {$[\varepsilon+1) !$} & $2 \varepsilon+00$ & $\theta E+(1)]$ & $6.9 E+i) 0$ & $0 E+00$ & $\varepsilon .\{E+\delta \hat{S}$ & $5 E+60$ & $E-64$ \\
\hline 180 & 170 & ib! & 157 & [46 & 140 & 135 & 150 & 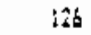 & 123 & 120 & 118 & 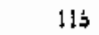 & 144 & is & $1: 1$ \\
\hline 20.) & 30.5 & 21.1 & 22.9 & 24.0 & 26.0 & 23.3 & $\$ 0.7$ & 弶. & $30 .:$ & 58.9 & 41.. & 74.7 & 47.7 & 51.9 & 5 \\
\hline $55+01$ & Et & $25+0$ & EE & $8 E+0 \vdots$ & +01 & +0 & $25+01$ & $15+81$ & JE+QLO & $1 E \div 00$ & $15+00$ & $6.25+300$ & $+5+0,0$ & $4.3 E+\% 0$ & $15+\infty$ \\
\hline L8: & $17 !$ & $16 t$ & 155 & !50 & 144 & 5 & $1: 5$ & 131 & ד] & 124 & [ี2 & 12i) & 5 & It & lis \\
\hline $2 j .5$ & 23.6 & 24.3 & 35.4 & 26.7 & 28.7 & 20.7 & 5.00 & 35.4 & 38.0 & 40.7 & 43.5 & $46 . \overline{5}$ & 49.2 & 26,2 & 5.2 \\
\hline 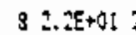 & $E+C$ & $E+0$ & {$[+01$} & 1 & $E+01$ & $3 E+0 !$ & [. $2 E+1] !$ & $0 E+0 !$ & $9,2 E+00$ & B. $\{E+00$ & $1.2 E+00$ & 6. $\left.3 E+0^{i}\right)$ & S.SE+\$O & 4. $a E+m 0$ & a, $2 E+D O D$ \\
\hline 180 & 172 & 165 & 159 & 152 & 147 & $1 \$ 3$ & 153 & LES & t2i & 8 & lat & 125 & $12 !$ & 619 & ב: \\
\hline 25.7 & 25.9 & 27.5 & 20.5 & 29.8 & 31.4 & 3.3 & 33.5 & 37.7 & Ho.l & 42.7 & 45.J & 40.1 & 5i). 9 & 5.7 & 56.7 \\
\hline+01 & $2.0 E+01$ & {$[E+0 \mid$} & $E+01$ & $\{+0\}$ & $E+01$ & $1.2 \mathrm{E}+0 \mathrm{I}$ & 1. $! E+0 !$ & 1. OE+O! & $9.1 E+10$ & B. $1 E+00$ & $1.2 E+00$ & $4,3 E+10$ & $5.6 \Sigma+70$ & $4.9 E+90$ & $4,2 \xi+010$ \\
\hline :BO & $1 \pi$ & 167 & ibl & ل & 190 & $14 b$ & 142 & 173 & 100 & $13 t$ & $1: 9$ & 126 & 124 & 122 & 124 \\
\hline$: 0.0$ & 30.2 & 36.7 & $3 i .6$ & 32.8 & 34.3 & 36.1 & 39.6 & 40.1 & $4 \mathbf{2} .4$ & 4.3 & 47.4 & 50.3 & 52.7 & 54.5 & 53.2 \\
\hline $5 E+101$ & $\{. j E+1\}$ & $S E+01$ & setot & $E+1) !$ & $j E+01$ & $1.2 E+1) t$ & $\{, \mid E+1)\}$ & $7.9 E+10$ & QE+ +ijo & $9.0 E+00$ & $7.25-04$ & 4. $. j E+130$ & $5.6 c^{2}+30$ & $4.9 e+90$ & 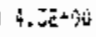 \\
\hline 192 & 174 & 163 & $16 j$ & 153 & IE & 149 & 145 & 141 & $\because z$ & ! 55 & $\mathrm{IJ2}$ & 129 & 127 & ב: & $\therefore$ \\
\hline JJ.2. & 30.5 & 34 & it & 25.9 & 7.3 & 38,9 & 40.7 & 42,7 & 44.8 & 97.1 & 47.5 & $52 .:$ & 54.7 & 57.3 & \pm 2 \\
\hline & 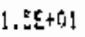 & & ZE+ & $\mathrm{EE}+01$ & $8 E+31$ & $5+0 !$ & IE+ul & $6 E+00$ & 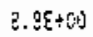 & $? E+C O$ & 1. IE+OS & a. $20+i b$ & 5.EE & $4.95+0,0 ;$ & $4,4 E+79$ \\
\hline :30 & 174 & 167 & ! & $i b j$ & 105 & I5! & {$[1]$} & $14 j$ & $1 \neq 0$ & 137 & 135 & $1 \approx$ & ! & $1 \hat{\imath} \hat{3}$ & $: 23$ \\
\hline 3.7 & Ib. $\hat{0}$ & 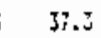 & 28.0 & 39.11 & 40,3 & 41.3 & 43.5 & 55.3 & 47.4 & 49.0 & 51.9 & 54.3 & 55.3 & 89.3 & 22.3 \\
\hline EE+OI & $B E \div 01$ & $1, a E+?$ & $2 E+? !$ & $1 c+01$ & $\therefore A E+31$ & $: .1 E+0:$ & EE +0 : & $(c+3)$ & 3. $3 \bar{c}+1 ; j ;$ & gE+90 & DE & e. $A[+\infty i\}$ & $5.5 E+1) 6$ & $7 \subseteq+100$ & 4.04 \\
\hline 120 & 175 & I0 & 165 & :\$1 & 157 & 193 & 149 & i46 & 143 & 140 & 137 & $\sin$ & 132 & 100 & 130 \\
\hline 40.11 & $40 . !$ & 49.6 & $\$ 1.2$ & $\$ 2.2$ & $43:=$ & 44,7 & 46.5 & 49.1 & 50.0 & 52.1 & 54.3 & 56.5 & $99 . \hat{0}$ & 61.5 & 54.3 \\
\hline $3 E+01$ & ה & & $: E+$ & 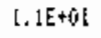 & $1 E+0$ & $55+91$ & $7 \varepsilon+00$ & $0 E+00$ & $B, J E$ & bE & $\theta E+C$ & !E+ & $5+30$ & $4,0 \mathrm{~g}+\mathrm{at} t$ & 4. \\
\hline II & 175 & $17 t$ & 1 & 16 & is & 155 & $15 !$ & 148 & 145 & 142 & 137 & 157 & 105 & $1 \pi$ & 130 \\
\hline 43.3 & 43.5 & 13.8 & 44.5 & $\$ 5.3$ & 46.4 & 47.7 & 49.2 & 50.9 & 52.7 & $5: .7$ & 56.8 & 59.0 & st. $\tilde{i}$ & 63.7 & ¿a. \\
\hline 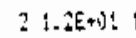 & SO & & f. & Etitil & . $0 E+01$ & $9 E+60$ & G. $3 E+00$ & a. $x+100$ & $8.0 E+00$ & 7. $(E+3)$ & t. $.5 E+00$ & $0.0 E+60$ & s.zE+jo & $E E+18$ & 4.2E+1, \\
\hline 180 & 175 & $17 !$ & 167 & 164 & 0 & 158 & 158 & 150 & 147 & 144 & 141 & 137 & 177 & $12 x$ & 18 \\
\hline 96.7 & 46.2 & 47.1 & 47.7 & 4.5 & 49.6 & 50.3 & 52.2 & $\$ 3.7$ & 5.5 & 57.3 & 59.3 & $\$ 1.5$ & 65.2 & bi & 6.4 \\
\hline$E+01$ & $1.1 E+0 \mid$ & $E+0\}$ & $\theta E+01$ & $2 E+04$ & $7 E+\infty$ & $3 E+00$ & $9 E+50$ & $3 E+00$ & $7 E \div 00$ & $i \xi+\geqslant 0$ & t5 & re & $2 E+90$ & $E+0$ & {$[E+2]$} \\
\hline 180 & 176 & 172 & 168 & ibs & I & 159 & $: 54$ & $15 !$ & 49 & :is & 143 & $14:$ & 177 & 136 & i: \\
\hline 5 & 50.1 & 50.4 & 51.0 & 51.7 & 52.7 & 53.9 & 5.2 & 56.7 & 58.5 & 60.1 & $(2.1)$ & $84 . j$ & 66.2 & 88.4 & 70.7 \\
\hline
\end{tabular}

Lung 0ose (rem) from Inhalation Estimated for 00002 May 7, 1986, for the Southeast Quadrant. Bearings are in Degrees and Distances are in Miles from Chernobyl. 


\begin{tabular}{|c|c|c|c|c|c|c|c|c|c|c|c|c|c|c|c|}
\hline \multicolumn{16}{|c|}{ 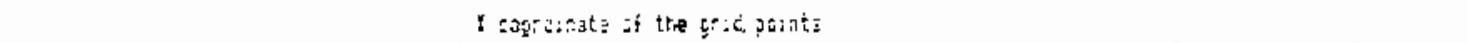 } \\
\hline+ & : & 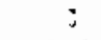 & ; & $s$ & b & $y$ & 3 & $\dot{y}$ & 10 & 11 & 12 & 13 & : & is & 10 \\
\hline & & 5 & 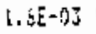 & t & . & $\{-1)$ & 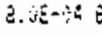 & $\hat{E} . \bar{Z}=-0.4$ & 7. $1 E-6:$ & $2.9 E-1: 4$ & $7,15-\hat{\imath} 45$ & 5. ละE- & J, & $7 E+i$ & \\
\hline 315 & & \pm 9 & 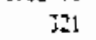 & $52 ;$ & 225 & 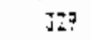 & $3: 1$ & int & היגוs & $34 i$ & $j 45$ & 348 & 32 & 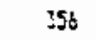 & \\
\hline $3 . .7$ & b. & 66.2 & bis & ย..) & bi.! & $5 \%,:$ & \$... & 5.2 & E.? & $z_{2} z_{i} ;$ & si. & $51,1)$ & 50.4 & iv. & 50.0 \\
\hline & 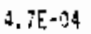 & -1 & 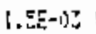 & $E-12$ & S & $\mathrm{E} \cdot) 4$. & $\varepsilon-\{4$ & $5-194$ & $4 E-94$ & $E-13$ & $E-\vec{v} \mid$ & $9 E-34=5$ & $\varepsilon-i\rangle$ & $\operatorname{CE}-1) 4$ & $E-44$ \\
\hline : & 314 & 317 & כ! & 321 & 324 & 327 & 30 & $33 j$ & SUt & 301 & 244 & 347 & $\because 1$ & 250 & \\
\hline 2.4 & 60.3 & b.:. & $5 ! .5$ & 59.5 & 57.2 & 5.5 .5 & 5.3 & 52.2 & $5 j .3$ & 45.6 & 49.5 & 47.7 & 47.1 & 9.8 & 46.7 \\
\hline$E-0 ;$ & $E-j$ & $E=04$ & $D E-93$ & $9 \varepsilon-8\}$ & $1.9 E-031$ & $1.25-0\}$ & $5-049$ & EE-134 & $9.75-04$ & $1 E-03$ & $15-153$ & $\{\leq-1)\}$ & QEE-14t 5 & $E-124$ & $18-14$ \\
\hline 3118 & 31. & 315 & 317 & 319 & $\approx 22$ & 35 & 322 & 331 & 35 & 378 & 342 & 377 & 51 & ?Ex & \\
\hline Ś..: & Eस.? & Si.j & (נ). & Só. 8 & 54.7 & $\Sigma i . i$ & 50.7 & 49.2 & 47.7 & 45.7 & $15, i$ & 44.5 & $4 j . z$ & 4.5 & 43.5 \\
\hline$i \varepsilon-1,4$ & $4,(E-1) 4$ & $E-14$ & 6.0E-..14 & {$[E-0]$} & I.IEAC & 2.0E-GS & $1.2 E-537$ & $9,2 E-04$ & $\mathrm{BE}-\mathrm{L}: 4$ & $i=-0]$ & $C E+2)$ & $E-5 j 5$ & $7 E-7+8$ & $4 t-14$ & $1 E-04$ \\
\hline 308 & 310 & 312 & 355 & 317 & 320 & 3.3 & 323 & 329 & 333 & 337 & 341 & 345 & 350 & 35 & i \\
\hline 64.0 & 61.5 & 59.0 & 56.6 & 54.5 & 57.1 & 50.3 & 48.1 & 46.3 & 14,7 & 45.3 & 42.2 & 41.2 & 80.5 & 4 & 40. \\
\hline CE-04 & $4 E+04$ & $\varepsilon-24$ & $E-04$ & $3 E-54$ & $1.6 E=032$ & $E-0 ;$ & $2.15-03 !$ & $1.2 E-03$ & $E-03$ & $1.2 E-0 j$ & $3 E-03:$ & $5-3\}$ & $2 E-4 \div 8$ & $E-04$ & $\{-\hat{p}+4$ \\
\hline उCb & 诲 & 210 & 312 & 315 & $3: 7$ & 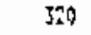 & inj & 327 & \pm 1 & 225 & 340 & 344 & 349 & 354 & \\
\hline 62.0 & 39.5 & 50.8 & $54 . j$ & 51.7 & 49.6 & 17.7 & $45 . j$ & 43.5 & th. & 40.3 & 39.13 & $\$ 8.0$ & sil. & 35.7 & 4.7 \\
\hline 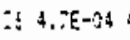 & {$[-1]$} & 174 & 5.124 & $15^{-i j 4}$ & $\varepsilon-\infty !$ & E-2 & {$[-0] 2$} & $E-1)$ & $2[-0]$ & $E E-9 j$ & $4 E-0 ;]$ & $5 E-03:$ & $4 E-0$ & {$[E-0]$} & -14 \\
\hline 305 & 305 & $30 ?$ & 399 & 312 & 315 & 319 & 321 & 325 & 329 & 3 & 30 & $3: j$ & 348 & 34 & \\
\hline ơ.! & 5.2 & 9.7 & $2 i . i$ & 43.6 & 47.1 & 44,7 & 42.7 & 4.7 & 3.9 & $\because 2.2$ & 5.5 & $5 \div 3$ & $\because:$ & M. & 3 \\
\hline$E-94:$ & $=-164$ & $E-34$ & $5-104$ & $\varepsilon-0\}$ & $E E-0+1$ & $5-0 j$ & $2 E-032$ & $z-0 j$ & $E+i j$ & $\{-0\}$ & $E-03:$ & $\{-0,5$ & $5-031$ & {$[i-4]$} & $5-54$ \\
\hline 300 & 347 & 304 & 30 & 209 & 31 & 315 & $2 ! 9$ & 322 & 325 & 愺 & " & 341 & $i$ & $\mathrm{IEJ}$ & 0 \\
\hline 88.3 & 55.5 & $\$ 2.7$ & 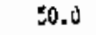 & 17.4 & 44.8 & 42.4 & 40.: & 38.4 & 80 & 34.3 & :2.8 & 31.6 & 50.2. & 4.2 & 50.0 \\
\hline 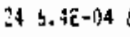 & 3. IE-DS & -04 & $A=-A 4$ & $\varepsilon-04$ & $7.1 E=64$ & $1.9 E-04$ & $E E-13:$ & {$[E-0]$} & {$[E-03$} & {$[-10]$} & {$[E-1)$} & $\sum E-\hat{\jmath} J 1$ & {$[E-1) ?$} & $E=05$ & $E-43$ \\
\hline 798 & 299 & 301 & & & 308 & 311 & 315 & 316 & & 327 & & 179 & & 392 & \\
\hline 56.7 & $\$ 3.7$ & 50.7 & 8.. & 45.3 & 42.7 & 0.1 & 37.7 & 3.7 & 3.5 & 3.4 & t. & 28.5 & $2 \pi$ & 26.9 & 23.7 \\
\hline$\{E-0\}$ & $5=-04$ & 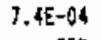 & -8 & 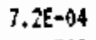 & SE-04 & $E-04$ & {$[-04]$} & $x=05$ & $25-6$ & $\mathrm{E}=-03$ & $E-$ & $3 E-032$ & E- & $7 E+15=$ & $E-23$ \\
\hline 295 & 286 & 298 & 30 & 2 & 304 & 307 & 311 & 5 & 319 & 324 & 300 & 336 & 344 & $35 t$ & \\
\hline 55.2 & 52.2 & 47.2 & 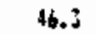 & 43. & 10.7 & 38.0 & IS. 4 & . & 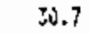 & 23.7 & 26.9 & 25.4 & 24. & 23.6 & 23.3 \\
\hline 032 & 2 & $=-193$ & $E-1) 3$ & $=-94$ & 3. $2 E-64$ & {$[-04$} & E-04: & $E+03$ & $\{x-03$ & $E-\hat{s}$ & $E-03]$ & $E E-032$ & $2.5 E-03$ & $2[-03$ & $1 E-93$ \\
\hline 291 & 999 & 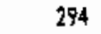 & & & & 303 & $T$ & & & 0 & & 3 & & \pm 50 & \\
\hline 8.7 & 5 & 47.7 & & 41.8 & 79.9 & 36.1 & 53.3 & 0.7 & 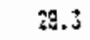 & 23.0 & 24,18 & $2 \pi+4$ & $2 \ldots+$. & 22,3 & $2 \hat{2} \hat{.}$ \\
\hline E & $3.95-1]\}$ & $z E-Q\}$ & " & $:(E-1)$ & $1, \pm[-1]=9$ & $?$ & $G E-031$ & $1.1 E-03$ & $E E-4 J$ & $[\varepsilon-1)\}$ & $5-103=$ & $(5-1) j j$ & $E-i z$ & $5-93$ & נ) \\
\hline 28 & & 291 & & 294 & & 799 & 302 & & & 715 & $\pm n 1$ & 399 & & 243 & i) \\
\hline 52.7 & $\$ 9.5$ & 16.4 & 43.5 & 40.3 & 31.3 & 34.2 & 31.4 & 28.7 & 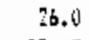 & 23.5 & 21. & 19.4 & 88.6 & $17 . c$ & 16.7 \\
\hline 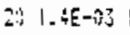 & 5,7 & $=-0$ & $15-07$ & - & SEAS & $5-1)$ & $E-n]$ & ; & $\varepsilon-$ & $E-15 ;$ & $E-1$ & $E-1$ & $\mathrm{E} E-1$ & $4,1 E-C$ & $15-3\}$ \\
\hline 334 & & 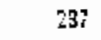 & & & & & 293 & 7099 & ה & 毦 & & $n$ & & 4 & \\
\hline 5.7 & 48.5 & $\$ 5.3$ & & 39.0 & & 32.3 & 27.8 & 9 & 67.8 & 21.5 & is. & ls.. & 24. & 5.7 & $\therefore$ \\
\hline (1) & & 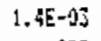 & & 7 & -07 & $\{-1\}$ & $=$ & $=0$ & 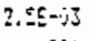 & 5 & 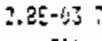 & $2 E-03:$ & E. & $S E-13$ & $E=0 j$ \\
\hline 28 & 28 & 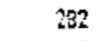 & & & & 3 & 230 & 3 & 905 & 300 & 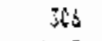 & its & $m$ & 1 & \\
\hline 51.0 & 7 & 44.3 & & & & 31.6 & .5 & 4 & 14 & .4 & 10.7 & 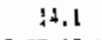 & 12.0 & $\hat{4.5}$ & 10.0 \\
\hline$=-104$ & $5-94$ & $\mathrm{E}=-1) 4$ & $E-03$ & $\{[-1\}]$ & $1.85-032$ & 2. $5 E-0 \hat{3}$ & $E-U 54$ & 4.!E-DJ] & 3. $2 E-9]$ & $9,4 \varepsilon+13$ & $4.5 E-65$ & $E=-1 ; \div 1$ & $1.5[-\sqrt{2} \mid$ & $1.45-62$ & 1., $35-12$ \\
\hline$\pi$ & 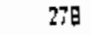 & 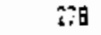 & & & & & & 5 & & 1 & & 303 & & 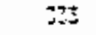 & a \\
\hline 顼.4 4 & $\$ 7.1$ & 43.8 & 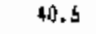 & $Y .3$ & 34.0 & 50.7 & 27.5 & 24.8 & 21.1 & $: 9.8$ & 19.9 & $12,1)$ & . & 7.5 & b. ? \\
\hline $5 E-04$ & $2 E-04$ & {$[-34$} & & $E-0$ & $E=-03:$ & $5 E-0 j$ & $E=032$ & $2.8[-0]$ & $5-0 j$ & 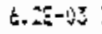 & 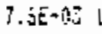 & $E-62$ & $5=17$ & {$[-1)^{\pi}$} & $E-12$ \\
\hline & & & & & & & & & & & & & & & \\
\hline 50.1 & & & & & & 00.2 & & 23.6 & & 17.0 & I & W.a. & $1 \%$ & .7 & 3.: \\
\hline & & & & & & & & & & & & & & 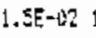 & $E-1 ; 2$ \\
\hline 270 & 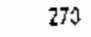 & 27 & & 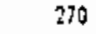 & & 270 & 270 & 270 & $\because$ & 270 & 270 & 270 & 270 & 270 & 0 \\
\hline 50.0 & 96.7 & 13.3 & $\$ 0.0$ & 35.7 & i..3 & 30.0 & 26.7 & 23.3 & 20.0 & 16.7 & iJ.: & 10.0 & s.7 & $\vdots . \dot{Z}$ & 4.9 \\
\hline
\end{tabular}

Residual Dose (rem/h) from Ground Contamination Estimated for 00002 May 7. 1986, for the Northwest Quadrant. Bearings are in Degrees and Distances are in Miles from Chernobyl. 


\begin{tabular}{|c|c|c|c|c|c|c|c|c|c|c|c|c|c|c|c|}
\hline & & & & & 20 & 2. & ari & 10ts & & & & & & & \\
\hline 1 & ? & $\therefore$ & 4 & 5 & $s$ & 1 & -1] & 7 & 1י & $1:$ & $: 2$ & $: Z$ & : 1 & 15 & is \\
\hline & & & $E+0$ & & E- & & $\therefore$ & 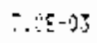 & $\cdots$ & se. & $=$ e. . & $\because-$ & $=$ & $\therefore \equiv$ & $=$ \\
\hline 245 & 2.5 & 65 & $\therefore 65$ & 284 & 264 & $2 t$. & 2s? & 25: & $1 \leq 0$ & SE & 9:E & $\because=:$ & 35 & 29 & $y$ \\
\hline 50.1 & 46.9 & 4.5 & t.: & $\ddot{0.0 . \bar{c}}$ & 5.5 & 50.2 & 35.9 & 3.3 & 0.2 & $17 . \mathrm{j}$ & 2.8 & 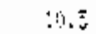 & 7.5 & 7.: & \\
\hline $\mathrm{AE}-\hat{\mathrm{v}} 4$ & -17 & {$[-1\}$} & -72 & $E-j 2$ & $E-10$ & -05 & $E-1 ;$ & $6 z-0 z$ & $a E-2 z$ & 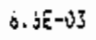 & $3 E-i$ & QE- & {$[2-6]$} & Q3. PE- & $E-4$ \\
\hline 262 & 261 & 251 & 25i) & 9 & 758 & 297 & 255 & 254 & $25 !$ & 248 & 243 & $23 \dot{a}$ & QNE & 206 & 100 \\
\hline E., & 47.1 & $1: .3$ & tit. 5 & : & $\geqslant 2.9$ & 30.7 & 2:. & 24.2 & 21.: & 18.4 & 14.9 & :2. & .4 & $i .5$ & $\cdots$ \\
\hline $1: 1.35-65$ & $2.0-05$ & Q. $\hat{E} E-\hat{i} \mathrm{~J}$ & $4 E-27$ & $A E-i_{2}$ & $9.7 \xi-03$ & $3.4 E-03$ & $\{E-J 3$ & $2 . J E-10]$ & $3.75-93$ & $4.5 E=0:$ & $9[-9]$ & $3.45-03$ & $1 E-22$ & 4 & {$[-8 ?$} \\
\hline $2 * 3$ & 257 & 257 & 255 & 254 & 25 & $\pi !$ & 249 & 24b & 243 & 277 & 23 & 225 & 713 & 198 & . \\
\hline $5:, v$ & $\$ 1.7$ & 44.5 & $4 ! .2$ & :3.. & $34 . \$$ & 31.5 & 29.5 & 25.4 & 2.4 & 17.t & 18.7 & 17.1 & 12.0 & $4 . Z$ & $\therefore$ \\
\hline SE- & $(t-1) 5$ & $12+1) j$ & $d$ & $\left(x^{2}-1,5\right.$ & $2.7 t+2$ & 1. TE-tis & $2,[E+1)]$ & ; & $0 E+2]$ & S.PE-1, & $5.1 E-65$ & $9,0 E-1, j$ & $T_{E-1) 2}$ & $4-3$ & $z E-\cdots$ \\
\hline $2 \pm 5$ & 254 & S5? & $2 \leq !$ & 75 & 248 & 146 & $2 \pm$ & 240 & 225 & $z:$ & ت & $6: 1$ & 205 & 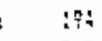 & 150 \\
\hline 51.7 & 48.5 & 45.3 & 43.2 & 57.0 & 35.9 & 32.8 & 27.3 & 23.8 & 24,2 & $2: .3$ & 13.7 & 16.7 & 4.7 & 13.7 & i.. \\
\hline & & 2. & -8 & {$[-10 \bar{J}$} & E & $2-\sqrt{3}$ & 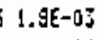 & $=-42$ & $82-03$ & 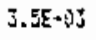 & !E-1] & $35-02$ & $2 \leq-\sqrt{2}$ & $F-02$ & $E-4$ \\
\hline $25 !$ & $2 \mathbf{E}_{0}$ & 243 & 247 & 245 & $27: 3$ & 240 & 237 & $2 \pi$ & 230 & 205 & 219 & 210 & Li! & $19 t$ & 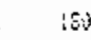 \\
\hline 5.7 & 49.6 & 46.4 & $4 . \Sigma$ & 40.3 & 3.3 & 34.3 & $\pm i,:$ & 28.7 & 36.0 & 23.6 & $\therefore 1.3$ & 25.7 & $1 E .0$ & ti. & S.? \\
\hline $101.1 E-03$ & t. $.1 \varepsilon-0 j$ & $1.1 E-0 J$ & $1 . \pm E-93$ & $j z-19 j$ & (. AE-1)? & $1 . \leqslant ฐ-4]$ & $1 . \pi=43$ & $2.1 E-6 z$ & $\varepsilon E-0 ?$ & SE-DS & {$[2-1]$} & $7,25-03$ & 9. $2 E-0 z$ & GE-12: & $G E-13$ \\
\hline 34 & 346 & 245 & $24 \pm$ & $2 \$ 1$ & 239 & 236 & 273 & 229 & 225 & 219 & 213 & 206 & 198 & $19 \%$ & : \\
\hline 3.7 & 50.9 & 47.7 & $\$ 4.7$ & 41.9 & 28.7 & isti & 3.3 & 30.7 & 23.2 & $26 . \hat{0}$ & 24.0 & 22.4 & 21.: & 20.3 & 20.0 \\
\hline $.9 E-24$ & $(E-1) J$ & {$[E-8]$} & $15 \cdot 63$ & ES-9J & 1..2E-0 0$\}$ & $2.55-0 ;$ & $7 E-43$ & $\{e-1)\}$ & $4 \varepsilon-0]$ & $65-33$ & $15-13$ & 4.3E-ij & $.7 \varepsilon-1\}]$ & $35-13$ & $a E-3$ \\
\hline 344 & 245 & 241 & 237 & 237 & 23 & 258 & 228 & 225 & 230 & 215 & 309 & 205 & 195 & $: 38$ & : 50 \\
\hline 55.2 & 52.2 & 49.2 & $46 . j$. & 43.5 & 40.7 & J9. & 35.4 & 33.0 & 90.7 & 29.7 & 36.9 & $25+4$ & $2 \$ . j$ & 23.6 & 23.5 \\
\hline SE-i) 4 & $Q E-0 z$ & $\{i-0\}$ & $1 E-9\}$ & $1,32-03$ & $7 \varepsilon-13$ & $1.52-43$ & $6 E-0 j$ & $9 E-19]$ & sE-0\} & $j E-0 z$ & $P E-0\}$ & $6-03$ & $3 E-03$ & $3 E+\hat{i n}$ & $(E-1)$ \\
\hline 2 & 210 & 238 & 236 & 200 & $2 \pm !$ & 228 & 228 & $22 !$ & $21 b$ & 212 & 206 & 00 & 94 & 197 & $: 5$ \\
\hline 5.7 & $53 . ?$ & 50.9 & 49.1 & 45.3 & 42.7 & 40 & $\pi$ & I5. 4 & 33.5 & 21.4 & 29.8 & 28.5 & 7.5 & 26.9 & 25.7 \\
\hline$E=04$ & {$[(-1)$} & $\{-0\}$ & 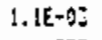 & $E=0 \vec{j}$ & EE-03 & -03 & $E=05$ & EE-0J & $15-02$ & $0 E-03$ & $9 E-03$ & $7 E-03$ & $S E-0]$ & -02 & $(\varepsilon-1)$ \\
\hline 27 & 3 & $2 \pi 5$ & Zנד & $2 \pi$ & 2.29 & 225 & 2 & $2: 1$ & : & 209 & 203 & 198 & 172 & 96 & 3 \\
\hline 58.5 & 5.5 & 52.7 & 50.0 & 47.4 & 44.3 & 92,4 & 10.1 & (e. 0 & 30.1 & 34.3 & 32.8 & 1.6 & 0.7 & 0.2 & 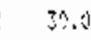 \\
\hline-04 & $9 E-194$ & $\varepsilon-0 J$ & $\{E-5 ;$ & $1.2 E-A Z$ & $1 . z \Sigma+\theta^{ \pm}$ & $1,4 E-0\}$ & $\Delta E-03$ & $E-0,4$ & $E$ E-dJ & $E-0 j$ & $\{\varepsilon-0]$ & $1\{-0\}$ & $2 E-03$ & (E--)Jj & $x-\therefore$ \\
\hline 27 & 274 & $23:$ & 200 & 227 & 275 & 221 & $?$ & $2: 4$ & 10 & 206 & 201 & 96 & 31 & 35 & (3) \\
\hline 50.1 & $5 i . j$ & 54.7 & Ex.: & 49.5 & 47.1 & 4.9 & 42. & 40.7 & 39.7 & 37.2 & TE.? & 4,3 & 4.3 & $3 \mathrm{~J} .5$ & 3.3 \\
\hline$x-1$ & $L-64$ & $=2$ & $15-13$ & S & S3 & 1. $4 E-0\}$ & $\tilde{E} E-1, j]$ & $75-13$ & $y=-i j$ & $\varepsilon-0 j$ & $E-15$ & J ב-E! & $\pm 5-\hat{6}$ & $\pi-3$ & ¿.SE- S J \\
\hline $2 \pi$ & $\because 1$ & $22 \hat{?}$ & 227 & 225 & 222 & 219 & 216 & 212 & 2193 & 204 & 199 & 95 & st & 15 & |SD \\
\hline S2. & 59.2 & 56.8 & 54.3 & $\$ 1, ?$ & 49.6 & 47.1 & 45.2 & 4.5 & e & 40.3 & 57.8 & 2.0 & 57.3 & $3 \dot{b} . \overline{5}$ & 6.7 \\
\hline & & & & $-n=$ & {$[-;)$} & & & $E-$ & & $\therefore \rightarrow 0$ & & {$[-1, j]$} & $T E-1$ & $\cdots$ & 距 \\
\hline 23 & $2=$ & 22 & 925 & 222 & $2 ! 9$ & 215 & 213 & 210 & & 202 & 198 & $: 94$ & 87 & 134 & $! 8$ \\
\hline 64.8 & bl.s & 59.9 & 56.6 & 54.3 & 52.1 & 50.0 & 48.: & 46.5 & 44.7 & 45.3 & 12.2 & $4: .2$ & $40 . \frac{1}{4}$ & (10.). & 40.0 \\
\hline $15-04$ & $9 E-04$ & {$[E-\Delta]$} & $5 \cdot 03$ & $\{20-0\}$ & $3 E-03$ & $1.4 E-0\}$ & SE-OS & $7 E-03$ & $8 E-0 ?$ & $E-Q j$ & $15-03$ & $1 E-0 ?$ & $1 E-0 z$ & $45-63$ & $\mathrm{BE}-\mathrm{S}$ \\
\hline $2 n ?$ & 317 & 225 & 222 & 220 & 217 & $2 ! 4$ & 211 & 208 & - & 201 & 197 & $19 n$ & 38 & lat & 150 \\
\hline $6 b .2$ & 63.7 & 51.3 & 59.0 & 56.8 & 54.7 & 52.7 & 50.9 & 49.2 & 47.7 & 46.4 & 45.3 & 44.5 & 43.9 & 4.5 & $\$ 3$. \\
\hline 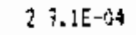 & & & & * & $E-0$ & & t- & 4 & E- & {$\left[-\mathrm{d}^{2} \mathrm{z}\right.$} & $2 E-19.3$ & FE- & 2. $5 \mathrm{E}-6 \mathrm{~J}$ & 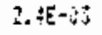 & . \\
\hline 22 & 2 & 2 & 220 & $2 ! 3$ & 215 & $2: 2$ & 209 & 206 & $2 n 7$ & 199 & 198 & 192 & 188 & 184 & 16 \\
\hline ba. 4 & 0 & & bl.s & 59.3 & 57.2 & 55.5 & 53.7 & 52.2 & 50.3 & $\$ 9.6$ & 49.5 & 47.7 & $47,:$ & 45.3 & 4t. \\
\hline 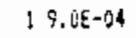 & & $E-0$ & $1 E-0 j$ & 1.2E-0 3 & $1.3 \mathrm{E}-03$ & 1. $+E-0 \Sigma$ & $1.5 E-0\}$ & !. $65-03$ & $75-03$ & $1.7 \mathrm{E}-03$ & 1. FE-03 & $2+4 E-03$ & $2.2 E-43$ & $2.2 E-53$ & 2. $45-3$ \\
\hline$z$ & 22 & 220 & 213 & itis & 213 & 210 & 200 & 305 & 201 & 190 & 194 & 131 & $: 97$ & نَ & 130 \\
\hline 70.7 & 68.4 & 56.2 & 64,0 & 62.0 & 60.1 & 58.5 & 56.7 & s5.2 & 53.7 & 52.7 & $5: .7$ & 51.0 & 50.4 & 50.1 & Ev. \\
\hline
\end{tabular}

\section{Residual Dose (rem/h) from Ground Contamination Estimated for 00002 May 7 , 1986, for the Southwest Quadrant. Bearings are in Degrees and Distances are in Miles from Chernobyl.}


a cectinate of the grid points

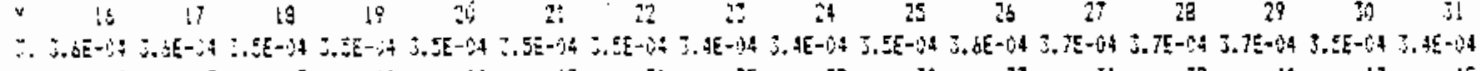

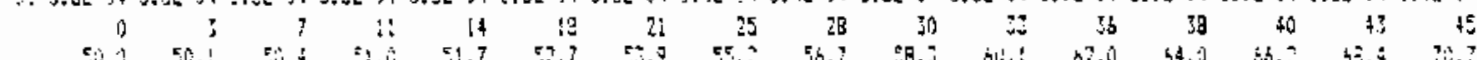

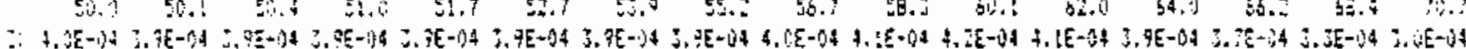

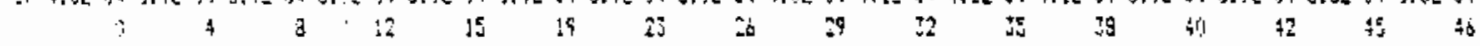

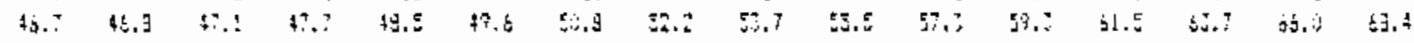

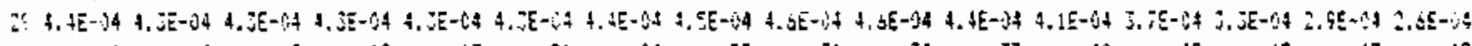

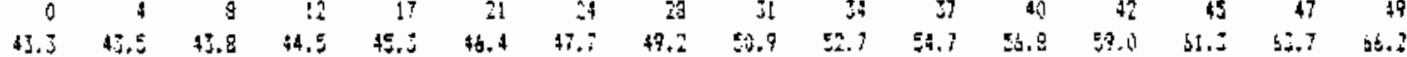

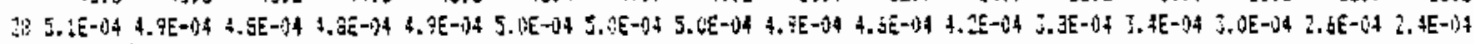

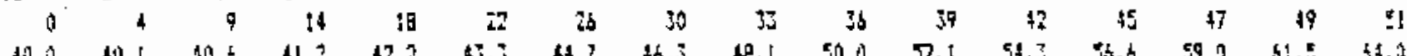

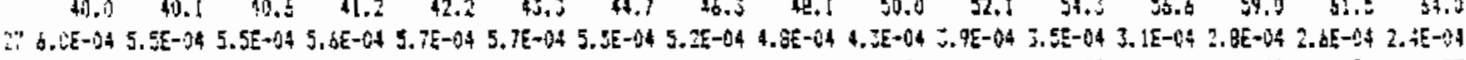

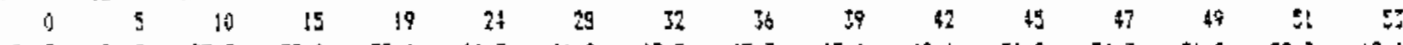

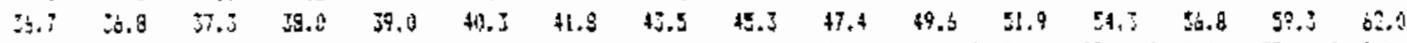

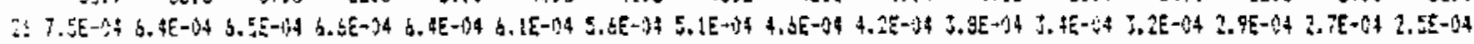

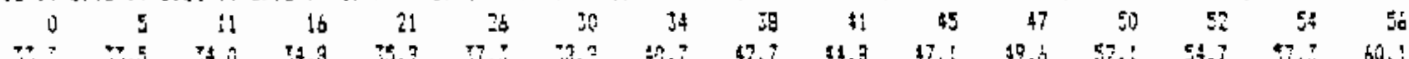

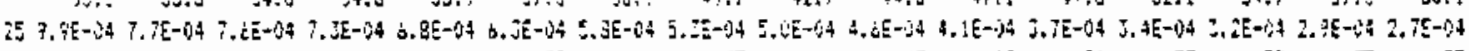

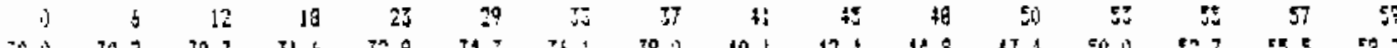

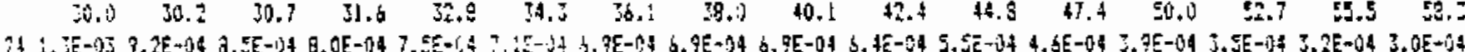

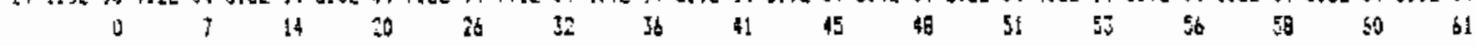

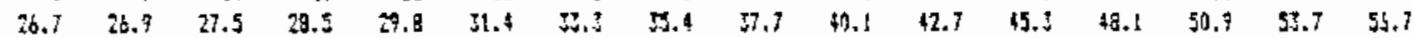

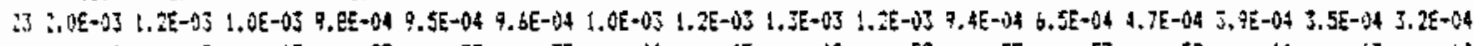

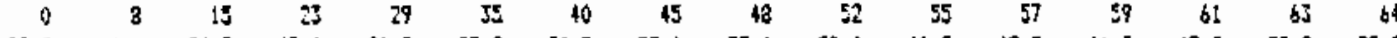

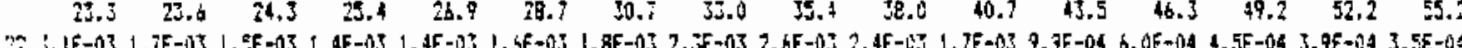

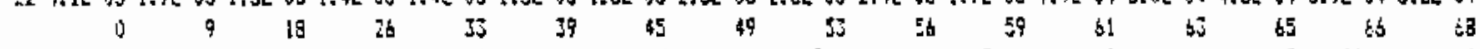

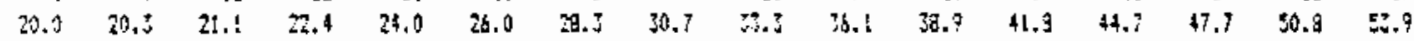

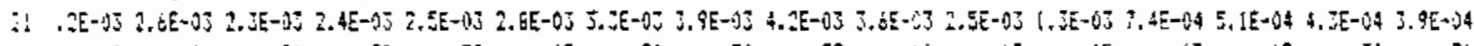

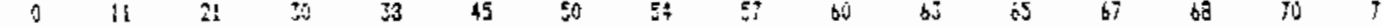

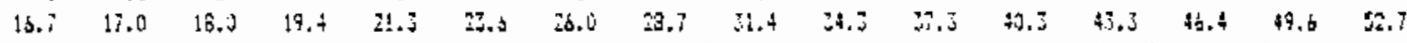

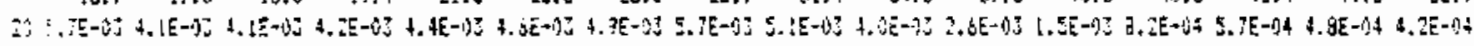

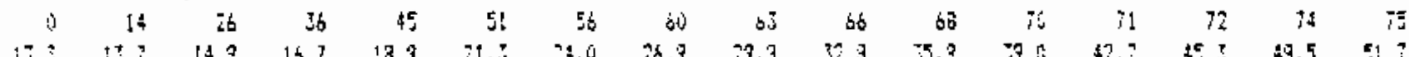

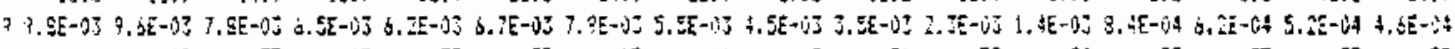

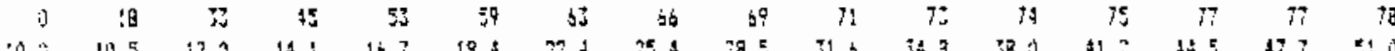

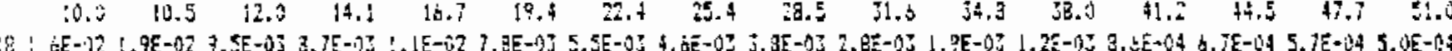

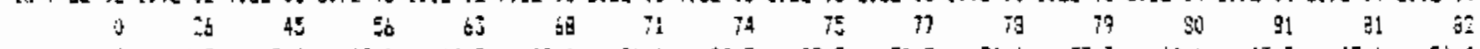

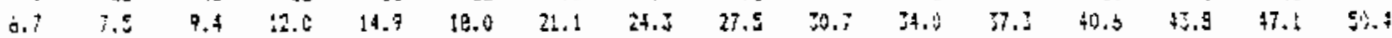

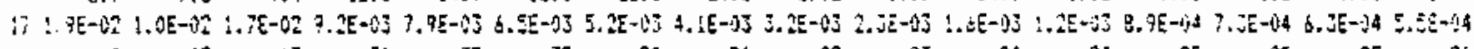

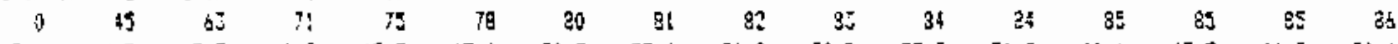

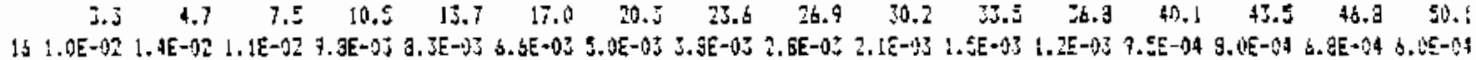

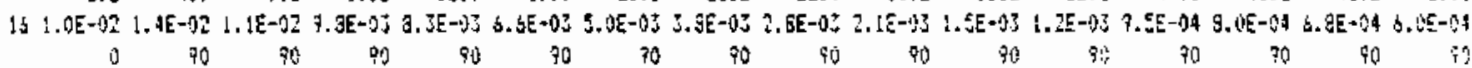

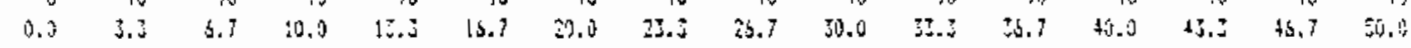

Residual Dose (rem/h) from Ground Contamination Estimated for $0000 \mathrm{Z}$ May 7, 1986, for the Northeast Quadrant. Bearings are in Degrees and Distances are in Miles from Chernobyl. 


\begin{tabular}{|c|c|c|c|c|c|c|c|c|c|c|c|c|c|c|c|}
\hline & & & & & ו & $s$ & 7 & & & & & & & & \\
\hline 16 & 17 & 19 & $: 7$ & $Z^{A}$ & 21 & $\because$ & $a$ & 24 & 25 & is & $\because$ & 25 & $?$ & ZA & $\because$ \\
\hline 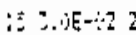 & 6 & $-x$ & $6 x$ & & & & $5-23$ & $E-6$ & & & & & & & \\
\hline 53 & 135 & 115 & 109 & $1: 4$ & 191 & ९९ & 93 & 97 & 96 & 95 & 95 & 94 & 34 & i4 & i \\
\hline : & 4.7 & 7.5 & 0.5 & 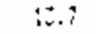 & 178 & 20.5 & is. & 35.5 & 20.2 & $\because .5$ & :i.j & 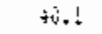 & +2...ّ & 46.3 & En: \\
\hline & & & & $(E-1) ?$ & -0 & 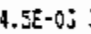 & -103 & $E-\phi$ & $E-13$ & $E-D z$ & $E-13$ & (1) & $E-4$ & & $9 E-104$ \\
\hline 180 & $15 J$ & 153 & 123 & 116 & $11 t$ & 168 & 105 & 104 & 192 & 101 & 100 & 99 & 49 & 98 & 97 \\
\hline 5.7 & 7.5 & 9.4 & 12.0 & 14.7 & 19.2 & 21.1 & 27.2 & 6.5 & s0.7 & 34.9 & $\because 7 . j$ & 40.5 & $\div .8$ & $47 . !$ & 50.4 \\
\hline $\bar{x}-1021$ & {$[E-22$} & $\varepsilon-12 z$ & $9[-1,2]$ & $3.9 E-935$ & $5.55-02$ & $j E-0 ;$ & $J E-0 z$ & $2.45-10 \mathrm{~J}$ & 2.tE-nz & $1,75-15$ & $1,15-35$ & $\therefore E-9 ; 1$ & 1.AE-0Z & $5 E-04 ?$ & $\nabla E-3 A$ \\
\hline$: 30$ & 161 & 145 & 135 & tiss & 120 & 116 & HIJ & 110 & 108 & 105 & 105 & 104 & 101 & $10:$ & :91 \\
\hline 10.13 & 30.5 & 22.0 & 14,1 & 16.7 & 19.4 & 22.7 & $=5,4$ & 29.5 & \$1.5 & IH. 8 & I..1] & $\$ 1.2$ & 44.3 & $\{\pi .7$ & $\because \therefore$ \\
\hline $1.2 E-\hat{G} 2$ & $6 E-122$ & $j E-9 x$ & TEE-0S & {$[E-1):$} & E-03 & $\{-0\}$ & DE- $Q S$ & SE- & $15-0 j$ & $3 E-131$ & SE-1): $:$ & $n E-19]$ & !E-i) & $\partial E-1$ & 9E-is \\
\hline 180 & 165 & 153 & 143 & ist & $! * 9$ & 123 & 119 & 116 & {$[15$} & iI! & 199 & tos & 107 & 205 & litt \\
\hline $1 \ldots$ & 12.1 & 11.9 & BS.? & 13.3 & $2 ! .3$ & 24.10 & $? 6.9$ & 29.2 & 22.3 & 55.9 & 39.0 & 42.2 & 15.2 & $\$ 3.5$ & Et.7 \\
\hline-6 & & & $E-j$ & ; & $E=93$ & {$[-03$} & $E-4 z$ & $25-8 j$ & $5 E-j 3$ & 1.8E- & $x[-2]$ & DE-6E: & {$[E-4]$} & $\leq \varepsilon-243$ & $45-34$ \\
\hline 180 & 158 & 153 & 119 & $14 !$ & 135 & 129 & 125 & 122 & 119 & 115 & 114 & 112 & 111 & 109 & 103 \\
\hline ?.ל. & {$\left[\begin{array}{l}1 \\
1.1\end{array}\right.$} & :9.9 & 19.4 & $2 \pi .3$ & İ. & 25.1 & 28.7 & 31.4 & 34.2 & 40 & 45,3 & 43.3 & 46.9 & 49.5 & $5 n .7$ \\
\hline $45-17=$ & $(E-1) 2$ & $E-62$ & S.5E-43 & $4.7 E-\Omega]$ & $E-1 ;$ & $3.8 \bar{E}=03$ & {$[-1) ?$} & {$[5-13$} & $.2 E-85$ & $.9 E-03:$ & L.SE +03 & $\therefore .3 E-93$ & {$[1,2 E-63$} & $9.75-64 \mathrm{~g}$ & $1.5 E-1) 4$ \\
\hline iso & 170 & $|b|$ & 15.5 & 246 & 140 & 185 & 150 & 125 & 123 & 120 & 118 & 116 & 114 & 193 & i1! \\
\hline$\therefore, 0$ & 20.5 & $2: .1$ & 22.4 & 24.0 & 24.0 & 29.3 & 80.7 & $\tau . Z$ & Ju. ! & 2.7 & 41.8 & 44.7 & 4.7 & 50.8 & 53.9 \\
\hline $2 E-23$ & $5 E-15$ & 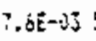 & $1 E-03$ & $e-n j$ & EE 95 & $5-43$ & $E E-0 z$ & $E-0, j$ & $2 E-2 \bar{j}$ & $B=0\}$ & $x-0 \div 1$ & $4 E-1]$ & $2 E-j]$ & $j E-633$ & $\mathrm{aE} \cdot 2 \mathrm{~s}$ \\
\hline 130 & 171 & 164 & 156 & 15 & 144 & 137 & $13 E$ & $13 !$ & {$[n]$} & 177 & $1: 2$ & 130 & 118 & t1s & $1: 5$ \\
\hline 23.5 & 23.6 & $24 . \overline{\mathrm{I}}$ & 25.4 & $26 . ?$ & 28.7 & N.? & 33.0 & X.t. & 38.0 & 40.7 & 4.5 & 46.5 & 98.2 & 32.2 & 5.2 \\
\hline$\varepsilon-0$. & $=-13$ & $A E-10$ & -133 & {$[-10\}$} & {$[-0,0]$} & E- & $E-$ & $(E-6)$ & 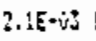 & {$[-0\}$} & $S E-O B$ & $4 E-2 \pi$ & IE-SI & $1,0 E-63=$ & $E-14$ \\
\hline 18 & 172 & 155 & 19 & 153 & 147 & 143 & 178 & 13 & $1 \pm 1$ & 128 & 126 & 123 & 121 & 119 & $1: 8$ \\
\hline 3.7 & 25.7 & 27.5 & 38.5 & 29.8 & 31.4 & 35.3 & 55.4 & 57.7 & 10.1 & 42.7 & 45.3 & 48.1 & 80.9 & 82.7 & 5.7 \\
\hline-07 & 03 & $E-0$ & $=03$ & $E-\lambda z$ & $1-2=03$ & $(6-0)$ & {$[\Sigma-05$} & $E-6 j$ & $1 E-35$ & {$[-0,3$} & $6 E-03$ & $\mathrm{E}-03$ & $24-03$ & 1.1E-0\} & $1.2 \varepsilon-04$ \\
\hline î & 17 & 167 & $16 i$ & 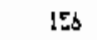 & 150 & 146 & 14? & : & 128 & $\{3\}$ & 129 & 126 & 124 & 122 & 126 \\
\hline 30.0 & 50.2 & 30.7 & 31.6 & 32.5 & 34.3 & ¿d. .1 & 38.0 & 40.1 & +2.4 & 44.8 & 47.4 & 50.0 & 52.7 & 55.5 & 59.5 \\
\hline $2 E-1 X^{*}=$ & $7 E-0 T$ & 4.1E-gZ & 3. & $\therefore+E-1, x$ & $2.9 \mathrm{E}-0 \mathrm{~J}$ & $2.7 E-5]$ & $2.5 \varepsilon-0\}$ & $j E-0 j$ & $E-0 ?$ & $\mathrm{G}=031$ & $(E-1):$ & $4 E-65$ & $2 E-0 ?$ & $1.1 E-\hat{-3} \bar{J}$ & $(x-54$ \\
\hline 150 & 174 & lica & 163 & ! $5 \theta$ & $15 \pi$ & 199 & 145 & 141 & 138 & [5 & i2 & $12 x$ & 127 & ! & Z: \\
\hline זי.בי & 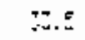 & 54.8 & 34.8 & $35 . ?$ & X7.3 & 8.9 & 40.7 & $\$ 2.7$ & 44.3 & 4.: & 49.5 & ร2.: & $54 . \vec{i}$ & 57.5 & 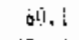 \\
\hline$(4,)^{-}$ & $7 E-2 J$ & 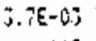 & $5-03$ & $3 .: 2 z-13$ & 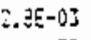 & $5-63$ & $F-15$ & $25-$ & ב & $5-9:$ & $(E=-1) ;$ & $4 E-0 j$ & {$[.2 E-4]$} & 1. $1 \mathrm{E}-\mathrm{iJ}$ & $\mathrm{JE}-: 4$ \\
\hline$\vdots$ & $: \because 4$ & 168 & $1: 54$ & : & iEs & $t$ & 197 & $\therefore$ & $1 \neq 0$ & 1.74 & 105 & 132 & 130 & $1: 2$ & 29 \\
\hline 3.7 & S.2.2 & 57.3 & 58.0 & 37.3 & 40.5 & 41.6 & $\$ 3.5$ & 45.3 & 47.4 & 49.5 & 5.9 & 84.3 & 5.2 & 59.3 & a2.? \\
\hline$E-4$ & 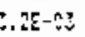 & "בני-عם & $E-1 \pm$ & I. $T E-A]$ & tE-j: & $+E+1) J$ & $E-03$ & $(E-1)]$ & $95-12$ & $7 E-A z$ & $5 E-103$ & $+E-i j J$ & $1 . .2 E-\hat{H}, 3$ & 1. $(E-1)=?$ & . DE- ? \\
\hline 180 & its & 17 & 165 & $15:$ & IE & is & 147 & $: 46$ & 145 & 140 & iJ & 178 & $: 2$ & 1.0 & $\therefore 2$ \\
\hline 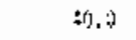 & 40.1 & 40.4 & $\$ 1.2$ & 12.2 & 43.2 & 94.7 & 46.7 & 48.: : & sti., & $52 .:$ & 54.3 & 85.6 & $9+0^{2}$ & $6: .5$ & 34.2 \\
\hline$A E-032$ & $9 E-0]$ & $z E-0 z$ & $65-63$ & 2. & $\{E-0\}$ & $35-0$ & $2 E-03$ & $E-43$ & $2 e-03$ & $7 E-05$ & $\{.5 E-3\}$ & $\mathrm{JE}-13\}$ & 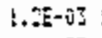 & $1.0 E+3=9$ & $25-\hat{n i t}$ \\
\hline ספ: & 175 & $! 7:$ & {$[6]$} & $t \dot{s}$ & 258 & & :s! & 14 & $! 15$ & $1: 2$ & $1: ?$ & 137 & 135 & 15 & 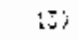 \\
\hline 43.3 & 43.5 & 4.8 & 44.5 & 45.3 & 45.4 & 47.7 & 49.2 & 50.9 & $52 . \vec{i}$ & 54.7 & 50.8 & 59.0 & 61.3 & 63.7 & E.: \\
\hline & $E-1 ;$ & 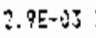 & $\varepsilon-?$ & , & $E=-4$ & $C E-C$ & $E-4 z$ & gE-Jj & $9 E-9 B$ & GE-DJ & {$[.5 \bar{E}-\hat{0}]_{4}^{3}$} & ב. 1. & I.XE- $\{?$ & $1 . J \mathrm{E}-\mathrm{i} ; \mathrm{Z}=$ & :DE- \\
\hline 18 & 175 & $17 i$ & 167 & ibt & 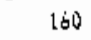 & 15 & 153 & 150 & 147 & 144 & $14:$ & $\therefore 9$ & 177 & 175 & ב:י \\
\hline 46.7 & 46.8 & $4 i .1$ & 47.7 & 18.5 & 19.6 & 56.3 & 52.2 & 53.7 & 55.5 & $5 i .2$ & 57.3 & 61.5 & $6: 3$ & a. & 83.t \\
\hline$[-1)]$ & 2. $45-0 \sqrt{3}$ & $E=-4 J$ & $2, x[-0]$ & 2. $.2 E-\downarrow j$ & {$[E-\sqrt{3}]$} & $\{-95$ & ż- & $2 E-03$ & $\{-43$ & $5-0 ;:$ & $\{E-13:$ & $\mathrm{CE}-10 \mathrm{~S}]$ & $i E-\nu$ & GE-U & $F-1 j q$ \\
\hline Ian & 173 & $17 ?$ & $! 58$ & 165 & ibi & 158 & 154 & LIt & 149 & $1 \div 6$ & $19 \vdots$ & $: 4 !$ & 179 & $: 36$ & 105 \\
\hline 50.0 & ED. & 50.4 & 51.0 & 51.7 & 52.1 & 53.9 & 55.2 & 56.7 & 59. & 50.1 & 52.0 & 84.0 & 85.2 & 33,5 & i10.7 \\
\hline
\end{tabular}

Residual Dose (rem/h) from Ground Contamination Estimated for 00002 May 7, 1986, for the Southeast Quadrant. Bearings are in Degrees and Distances are in Miles from Chernobyl. 
No. of

Copies

OFFSITE

2 DOE/Office of Scientific and Technical Information

ONSIIE

DOE Richland Operations Office

\section{E. C. Norman}

16 Pacific Northwest Laboratory

W. E. Davis

J. W. Falco

J. M. Hales

P. C. Hays

J. M. Hubbe

E. L. Owczarski

J. V. Ramsdell

R. L. Skaggs

J. A. Stottlemyre

$R$. E. Wildung

Pubiishing Coordination

Technical Report Files (5) 
